
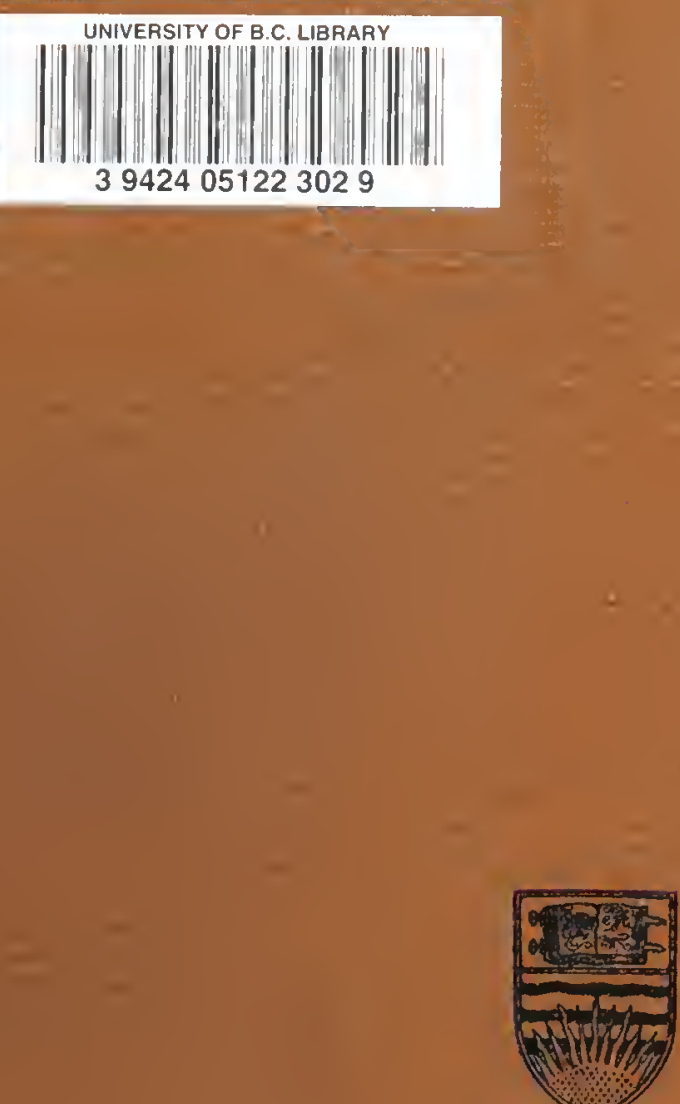
THE LIBRARY

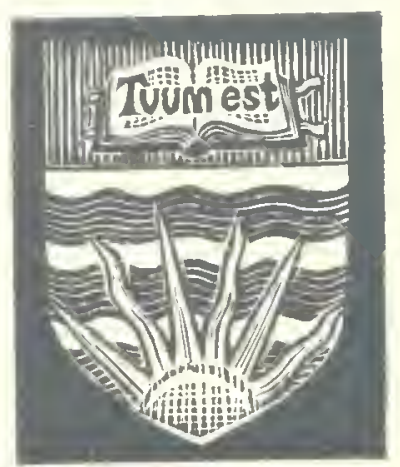

THE UNIVERSITY OF BRITISH COLUMBIA 
Digitized by the Internet Archive in 2010 with funding from University of British Columbia Library 



\section{LA FORETT DE LA COTE D'IVOIRE \\ et}

SON EXPLOITATION 

VARIÉTÉ D'HCAJOU

\section{DE LA COTE D'IVOIRE ET SON EXPLOITATION}




\section{PUBLICATIONS AFRICAINES}

38. Rue du Mont-Thabor, PARIS

\section{La Forêt de la Côte d'Ivoire}

ET

\section{SON EXPLOITATION}

\section{Par M. Jean MENIAUD}

Administrateur des Colonies. - Ex-chef du Service Forestier de la Colone.

Précèdé d'une Préface de M. le Gouverneur ANTONÉTTI

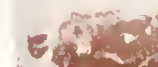

(c) 3 ?
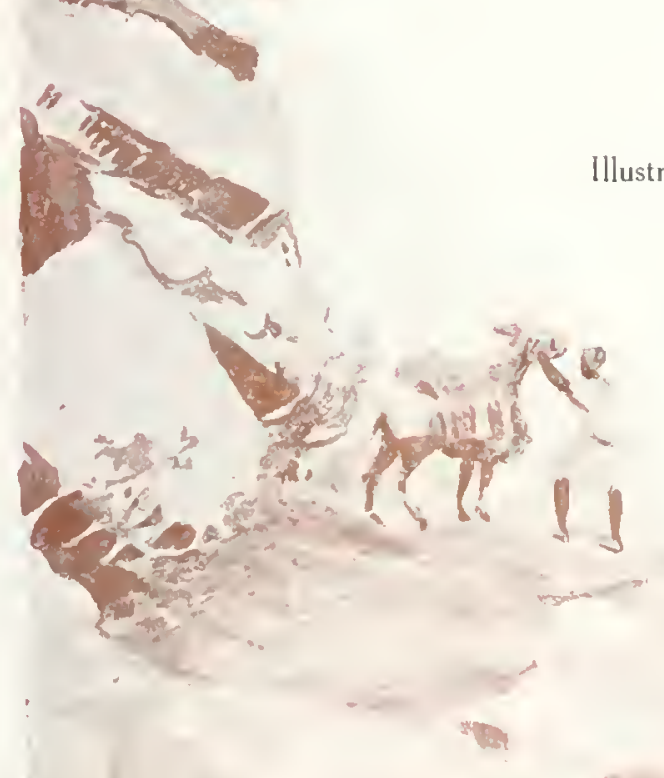

2.2
D'une Introduction et de Considérations génèrales sur le payss et les habitants de M. M. Larre

Illustrations de M. Lous DUPUIS. - Bayonne (B.-P.)

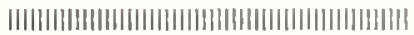





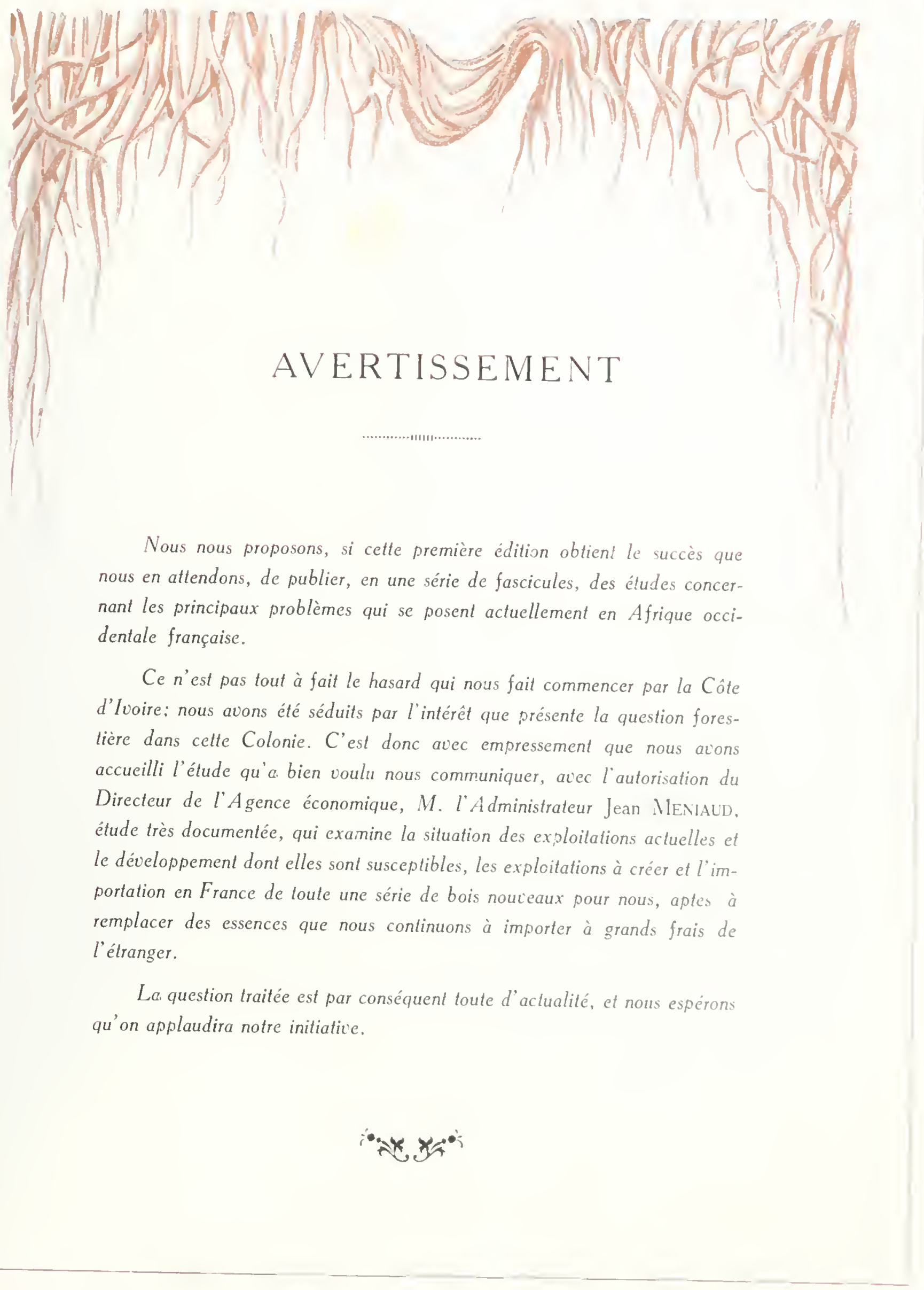





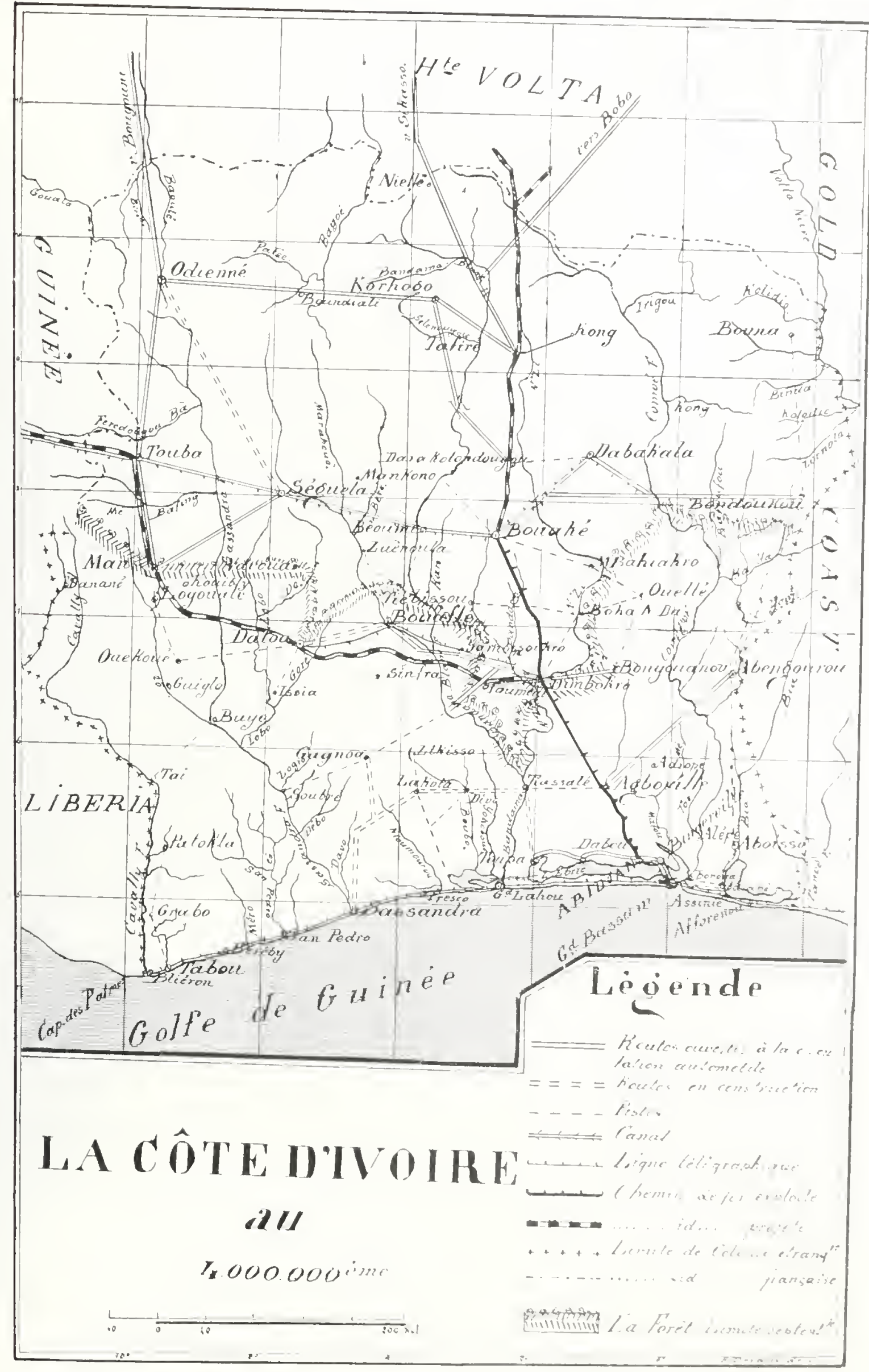





\section{PRÉFACE}

Il a fallu la guerre pour que la France appréciât la valeur de l'Empire Colonial qu'elle avait constitué en 30 ans. Contrairement à beaucoup de prévisions, non senlement il ne lui a créé aucune difficulté au cours de la tourmente, mais il a été l'un des facteurs de la victoire en apportant à la France l'appoint de 250.000 soldats, de 550.000 travailleurs et d'approvisionnements considérables dont la valeur se chiffrant par des milliards de francs n'a pas, en augmentant nos dettes à l'extérieur, pesé sur notre change.

Cette aide, les Colonies penvent nous la continuer, plus efficace encore, an cours de la paix si semblable à la guerre que 4 ans de destruction de vies et de richesses, ont imposée au monde. La barrière des changès qui n'est pas une des moindres régressions amenées par la guerre, oblige chaque pays à chercher les moyens de se suffire dans les limites de ses propres frontières. La France est à ce point de vue une des nations les plus favorisées, car elle forme à elle seule un organisme économique presque complet. Il ne lui manque que des matières premières en petit nombre, et elle pourra les tronver dans ses colonies:

Le coton qui lui sera fourni par le Sondan;

Le pétrole que rempliceront l'alcool et les matières grasses que ses colonies pentent fournir à profusion:

Les bois dont la Côte d'Troire et le Gabon contiennent des résert'es qui, sagement exploitees, sont pratiquement inépuisatles. 
La mise en valeur de notre domaine colonial est donc aujourd'hui une question nationale. En prenant sur notre propre sol ce que nous demandons actuellement à l'étranger, nous libérons nos changes. Au lieu de nous appauvrir, nos besoins en matière première nous serviront à féconder un domaine aujourd'hui français. En enrichissant nos populations indigènes, nous les élcverons, car l'histoire nous enseigne que la civilisation la plus élevée ne s'est jamais rencontrée que chez des nations riches.

Le gros obstacle à la vente des bois coloniaux est qu'ils sont mal connus, ou mieux pas connus du tout en dehors de l'acajou et de l'ébène; mais ces deux essences ne représentent qu'une infime partie de la richesse de nos forêts coloniales. Celles-ci contiennent non seulement des bois équivalents à tous ceux dont se sert l'industrie curopéenne, mais encore des essences ayant des qualités spéciales qui permettront de créer des industries nouvelles.

Toutefois ce trésor immense était mal connu. La grande forêt équatoriale se défend farouchement par ses marais, ses fourrés souvent inextricables, la masse de ses arbres foudroyés dont chacun forme un mur. La prospection y est pénible, l'identification de centaines d'espèces inconnues hérissée de difficultés et malgré les beaux travaux de la mission Bertin, des professeurs Perrot et Chatelard, du professeur Chevalier, nous avons encore bien des découvertes à faire; des surprises heureuses à espérer.

Le travail est cependant déjà assez avancé pour que l'on puisse présenter au grand public une vingtaine d'espèces dont les qualités et les usages sont aujourd'hu: bien déterminés.

Tel est le but de l'ouvrage de M. l'Administrateur Jean Meniaud, ancien chef du Service Forestier à la Côte d'lvoire. C'est une ceuvre de grande vulgarisation. Elle a eu la rare fortune de trouver un éditeur soucicux de bien la présenter, et cela encore est un signe des temps nouveaux qui semblent devoir luire pour les Colonies.

Je terminerai par des chiffres; n'est-ce pas par eux que tout se traduit actuellement. Ils montreront l'importance qu'a pour la France l'exploitation de ses forêts coloniales.

La France consommait avant la guerre 12.000 .000 de mètres cubes de bois dont 4 millions de mètres cubes provenaient de l'étranger. La dévastation de ses forêts au cours de la guerre a réduit leur production à 6 millions de mètres cubes, alors que la nécessité de réparer les ruines a augmenté nos besoins. L'écart entre ceux-ci el notre production représente donc environ 8 millions de mètres cubes. Au prix actuel des bois, c'est un trihut annuel de 4 à 5 milliards que nous avons à payer à l'étranger. L'exploitation de nos forêts coloniales peut nous en libérer. Ces chiffres montrent l'importance nationale de la question traitée par M. Jean Meniaud.

Paris, le 10 Mai 1922.

\author{
R. ANTONETTI, \\ Gouverneur de la Cóte d'Ivoire.
}




\section{INTRODUCTION}

Par ce que nous en connaissons, la Côte d'lvoire nous apparaît, non seulement comme l'une des plus riches Colonies françaises, mais encore comme l'une des contrées du monde les plus favorisées par la nature. Riche d'avenir, car à la faveur du climat chaud et humide dont elle jouit, son sol, extrêmement fertile, convient à toutes les cullures tropicales qui font la forlune des lles de la Sonde, des Indes Britanniques, des Antilles et de l'Amérique centrale. Riche de présent aussi, car elle possède une forêt admirable dont l'exploitation n'est pour ainsi dire qu'à peine ébauchée et qui pourra alimenter des industries considérables.

Nous sommes heureux d'avoir l'occasion de faire connaitre cette richesse, le parli qui pourra en être tiré lorsque, mieux éclairés, des capitaux, des énergies nouvelles consentiront, en bénéficiant de l'expérience acquise, de la mise au point de questions qui ont demandé plusieurs années de recherches, d'essais et de tâtonnements, à aller faire en grand ce qu'ont réussi à faire en petit les premiers pionniers installés là-bas, pionniers venus un peu par le fait du hasard. sans programme préalable, sans capitaux pour la plupart, dans un pays à peine conquis et dont ils ne connaissent rien. Et pourtant l'œuure de ces pionniers, pour n'être pas parfaite, est déjà considérable, surtout si l'on réfléchit aux difficultés qu'ils ont dû surmonter.

Il ne faudra pas cependant que les nouveaux venus apportent avec eux cet esprit étroil qui is rencontre malheureusement trop souvent dans les entreprises coloniales, et dont la caractéristique est de vouloir obtenir des avantages immédiats avec un minimum de risques, un minimum d'efforts. Des fortunes rapides sont possiblos à la Côle d'lvoire. Nous en alons connu quelques exemsles ces dernières années. Mais faisons en abstraction et disons au contraire que la plupart des affaires à entreprendre à la Colonie, que ce soit dans l'A griculture, dans l'Industrie ou l'cxoloitation forestière, sont des affaires de longue haleine qui ne peuvent donner de résultats qu'au bout de plusieurs années de labeur et de persévérance.

On remarque que $M$. MENIAUd ne fait l'apologie d'aucune entreprise particuliere, de réclame pour aucun des groupements ou Sociétés ayant déjà des intéréts à la Colonie ou suscestibles d'en aroir par la suite. Nous lui laissons du reste la responsabilité des indications qu'il donne. nous-mêmes ne faisant que présenter son travail au public. 
Ce travail est très complet, très documenté. Quoi qu'il en soit, il ne peut avoir qu'une portée trop générale pour dispenser quiconque désirera entreprendre là-bas une exploitation forestière, de procéder à une étude détaillée, minutieuse, des conditions dans lesquelles cette entreprise devra être organisée, puis d'établir un programme qui tiendra comple de tous les facteurs d'insuccès. Il est indispensable, pour cela, d'aller sur place et de consacrer à ce premier travail le temps et les soins nécessaires.

Nous avons voulu et nous ferons notre possible pour continuer à offrir au lecteur une édition luxueuse, de façon que commerçants, industriels, hommes d'affaires, conservent assez longtemps sur leur table l'exemplaire qu'ils se seront procuré, qu'ils fuissent, ayant un certain plaisir à l'ouvrir, le lire et le relire, bien s'imprégner de cette idée que la France possède, dans ses Colonies, un champ d'activité considérable pour ses enfants, des richesses qui n'attendent que des colons et des capitaux pour être exploitées ou mises en valeur.

Nous aimons à croire que notre effort ne restera pas stérile. Quelque maigre que puisse êtrc le résultat, s'il en est un, nous nous estimerons satisfaits. Notre ambition ne va pas plus loin.

M. L. 


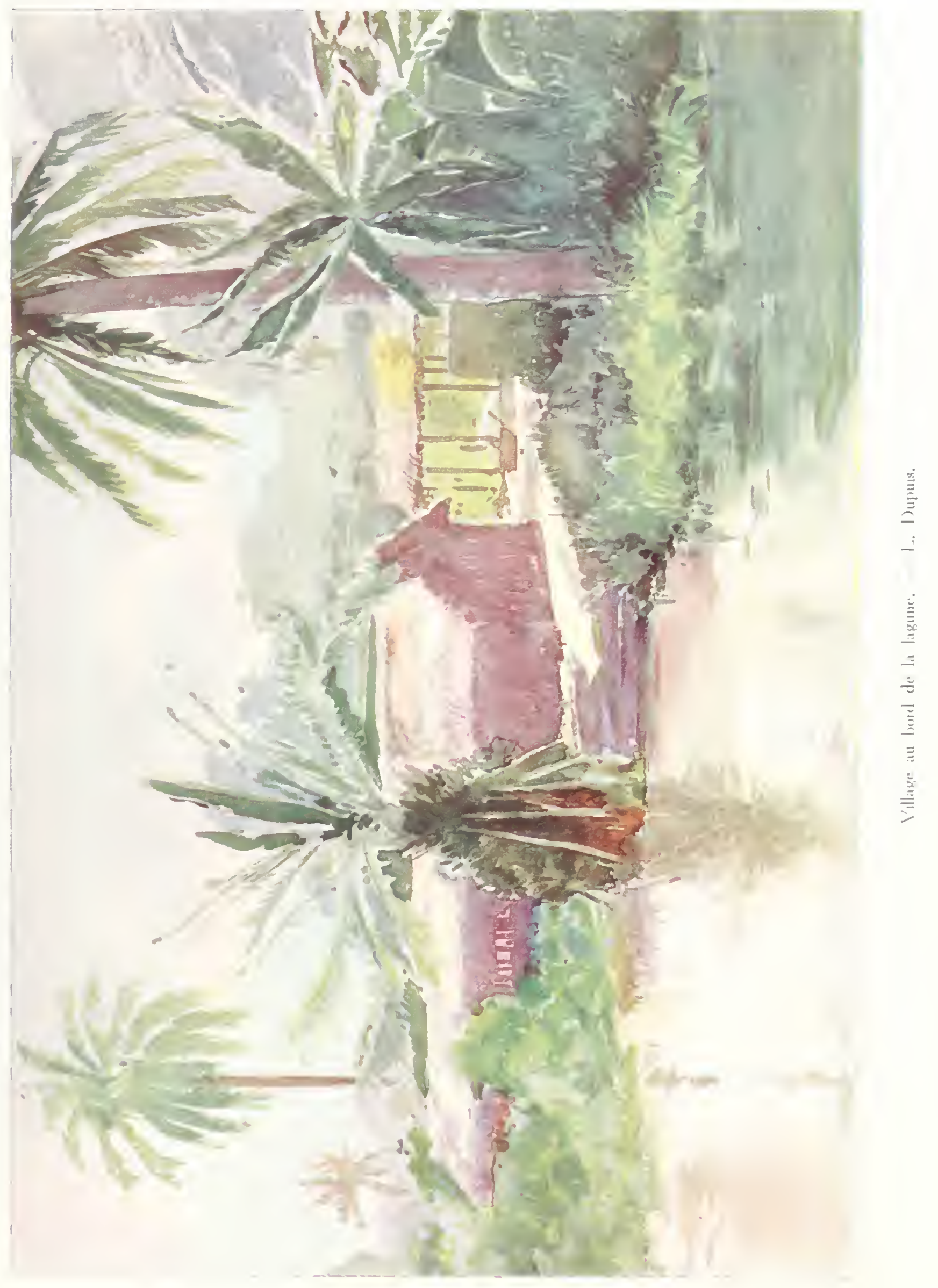




\section{LA COTE D'IVOIRE}

Considérations générales sur le Pays et ses Habitants

M. M. LARRE

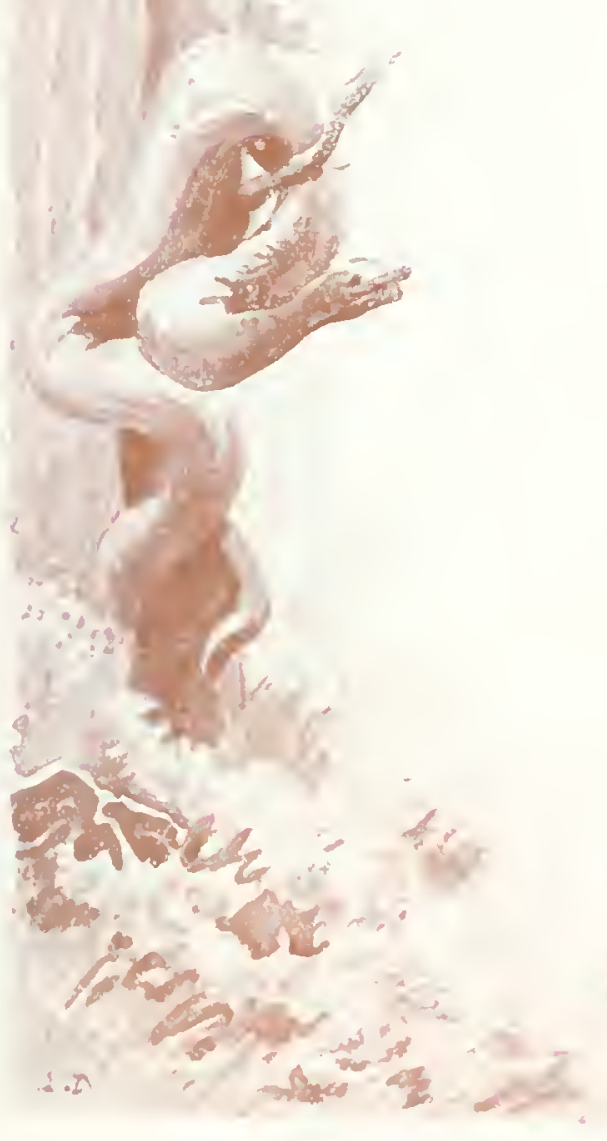




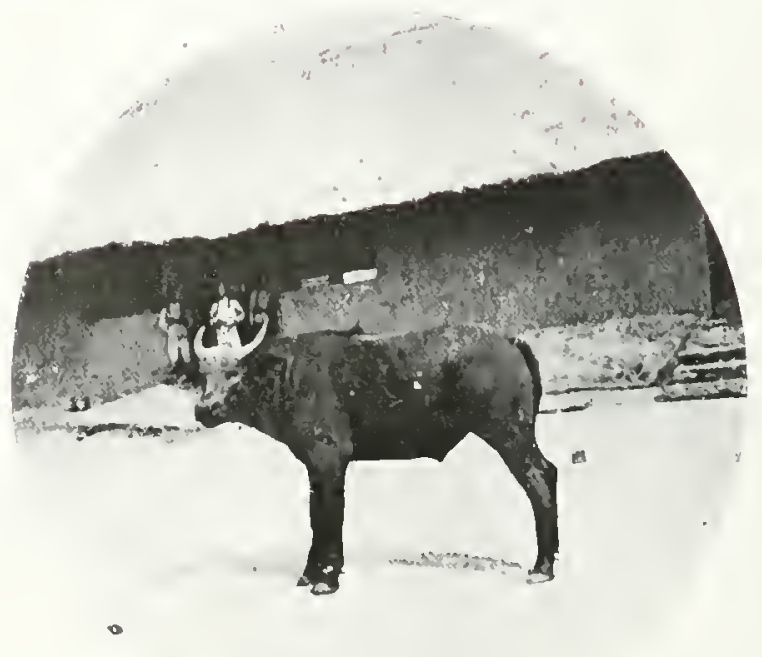

Jeune buffle.

Cliché Burger 


\title{
LA COTE D'IVOIRE
}

\section{Considérations générales sur le Pays et ses Habitants}

\author{
par M. M. LARRÉ
}

Qu'on nous permette tout d'abord, puisqu il va être question de la Côte d'Ivoire, de donner, dans ce premier fascicule, quelques éclaircissements sur la géographie, le climat, la faune, la flore, les habitants du pays, le commerce et

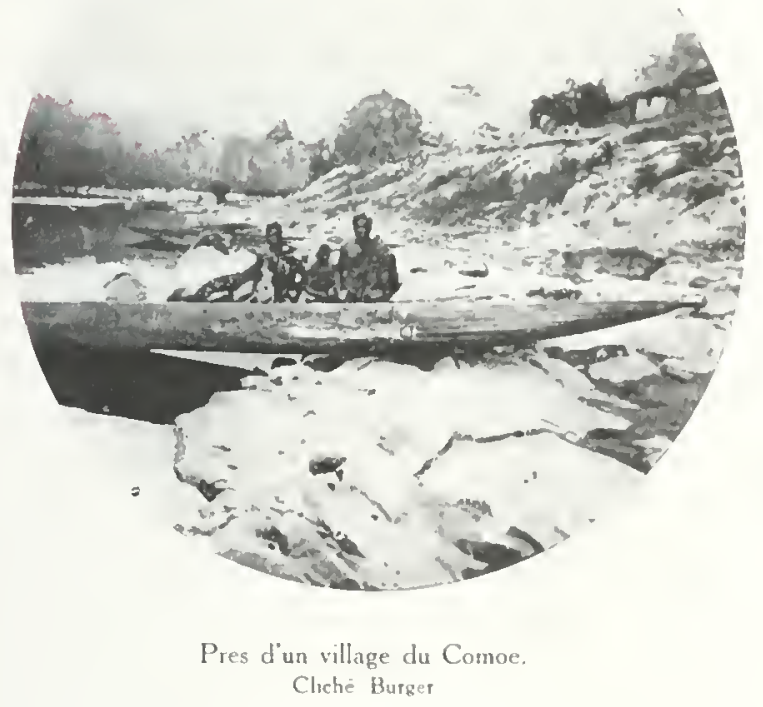

l'état actuel de la colonisation. Nous n'examinerons que très succinctement chacune de ces questions, priant le lecteur de se reporter, pour plus amples informations, aux ouvrages déjà parus sur la matière et dont nous publions la liste à la fin de celte publication.
Nous passerons après à la question forestière que M. Méniaud expose magistralement.

\section{GÉographie}

La Côte d'lvoire a une superficie d'environ 315.000 kilomètres carrés. Située entre les 5 et $10^{\circ}$ degrés de latitude Nord, et 5 et $11^{\circ}$ degrés de longitude Ouest (Méridien de Paris). elle affecte la forme d'un quadrilatère presque régulier. Au Sud, elle est limitée sur 550 kilomètres par l'Océan Atlantique (Golfe de Gui. née) : à l'Ouest, sur 630 kilomètres par le Libéria et la Guinée Française: au Nord. par le Soudan Français et la Haute Volta: à l'Est, par la Colonie Anglaise de Gold Coast.

Avec le Sénégal, la Mauritanie. la Guinée. le Soudan, la Haute Volta, le Dahomey et le Territoire Militaire du Niger, elle constitue la Fédération de l'Afrique Occidentale Française dont le siège (Gouvernement Général) est à Dakar.

La Côte d'lioire se divišc, à peu près par moitié, en deux zones bien distinctes: la zone sylvestre, au Sud, et la zone des savanes au Nord de la Colonie. Ces deux zones. très différentes au point de rue des productions, diffèreni également comme climat, température. époques des chûtes d'cau, etc... Elles diffèrent mème comme populations. question de races mises à part. 


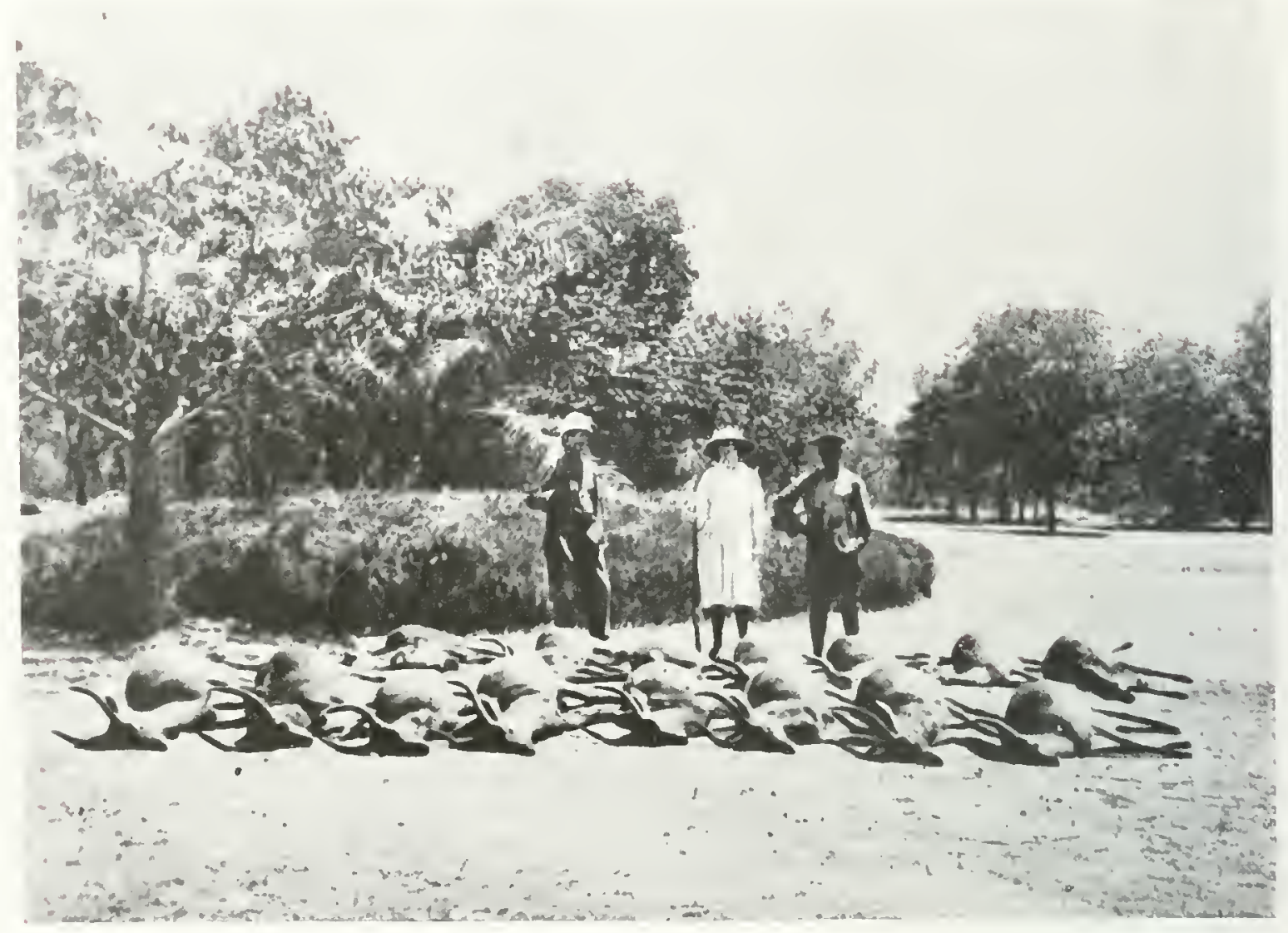

Tableau de Chasse.

Clichè Burger

les habitants des savanes étant plus accueillants, plus exhubérants, moins primitifs en somme que ceux qui sont habitués à vivre sous le couvert de la grande forêt.

\section{Orographie - Hydrographie}

La Côte d'lvoire offre, dans son ensemble, l'aspect d'une vaste plaine légèrement inclinée du Nord au Sud. La déclivité est assez régulière depuis les confins du Soudan jusqu'à la mer et n'est coupée transversalement que par de petites dépressions où serpentent et coulent en cascades les affluents des rivières importantes, ces demières coulant d'une façon générale dans la direction Nord-Sud. A Bouaké, à 300 kilomètres environ à vol d'oiseau de la mer. l'altitude est de 400 mètres. La pente moyenne serait, par suite, de $1^{\mathrm{m}}, 33$ par kilomètre pour la partie comprise entre Abidjan et Bouaké et que traverse la voie ferrée qui monte vers le Nord.

Comme massif montagneux, il n'y a guère lieu de signaler que celui de Touba-Odienné, au Nord-Ouest de la Colonie, massif qui est la continuation de celui qui prend naissance dans la Basse-Guinée et traverse le Sierra-Léone et le Libéria. On trouve dans la région de Touba des dômes et pics atteignant de 1.000 à 1.600 mètres.

Le petit massif central du Baoulé entre le $\mathrm{N}^{\prime} \mathrm{Zi}$ et le Bandama, ne présente guère de sommets dépassant 300 mètres ; il en est de même des collines de Boudoukou, au Nord-Est de la Colonie.

Quatre fleuves principaux et presque parallèles draînent les eaux vers l'Océan : le Cavally, à l'Ouest, qui sert de frontière sur la partie 
inférieure de son parcours avec le Libéria ; puis, en allant vers l'Est: le Sassandra, fleuve assez accidenté qui prend sa source dans la région d'Odienné : le Bandama qui baigne la région de Korogho, au Nord, reçoit d'importants

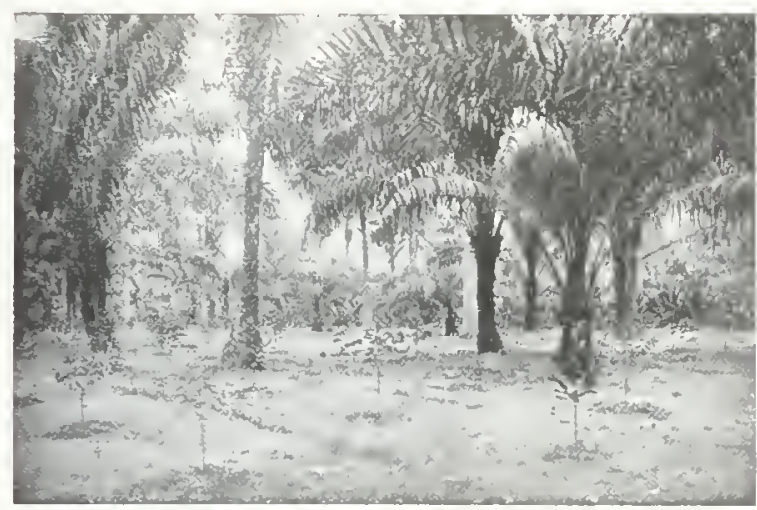

Palmeraie naturelle amenagee abritant une plantation de caféiers àzés d'un an.

Cliché Agence économique A. O.F

affluents, dont le $\mathrm{N}^{\prime} \mathrm{Zi}$, et se jette dans l'Océan près de Grand Lahou; le Comoë, dont la source voisine avec celle du Bandama, traverse la région de Kong et vient déboucher à GrandBassam.

Comme fleuves secondaires : la rivière Tabou et le San Pedro qui se jettent directement dans l'Océan près des localités du même nom; la Niouniourou qui alimente la petite lagune de Fresco ; le Goboua et le Yocoboué qui débouchent dans la lagune de Lahou; l'Agnéby qui débouche dans la lagune Ebrié : la Mé dans la lagune M'Potou : la Bia dans la lagune Aby ; le Tanoë qui vient de Gold Coast et sert de frontière avec cette Colonie sur une trentaine de kilomètres.

Tous ces fleuves et rivières, sinueux et torrentueux, ne présentent qu'un intérêt assez minime comme moyens de communication. Le Comoë n'est navigable, en partant de la Côte, que sur 60 kilomètres environ; le Bandama également, encore pour des vapeurs d'assez faible tonnage. Partout, les rapides succèdent aux rapides, rendant impossible, en certains endroits, le passage des pirogues indigènes. Sur le Comoë, le Bandama et le Sassandra, il existe toutefois entre les seuils, dans la partie moyenne de la Colonie, des biefs plus ou moins longs, utilisables éventuellement pour le passage d'embarcations de 20 à 50 tonnes. Le Cavally serait navigable jusqu'à une centaine de kilomètres de la Côte : de petits vapeurs pourraient le remonter assez loin. Malheureusement, la région qu'il traverse, aussi bien du côté français que du côté libérien, a été encore bien peu pénétrée. Les transactions commerciales y sont presque nulles.

Fleuves et rivières de la région côtière sont utilisés pour le flottage des bois, acajou notamment, destinés à l'exportation. Le régime de la plupart de ces cours d'eau est très irrégulier. A sec pendant une partie de l'année, leur lit se remplit subitement au moment des grandes pluies et se transforme en torrents qui débordent parfois largement. C'est le moment que choisissent les exploitants forestiers pour acheminer vers les lagunes les billes de bois rassemblées auprès des rivières pendant les mois qui précèdent l'hivernage.

\section{Clinatologie}

La Colonie, bien que déjà éloignée de l'Equateur, jouit, tout au moins en ce qui con-

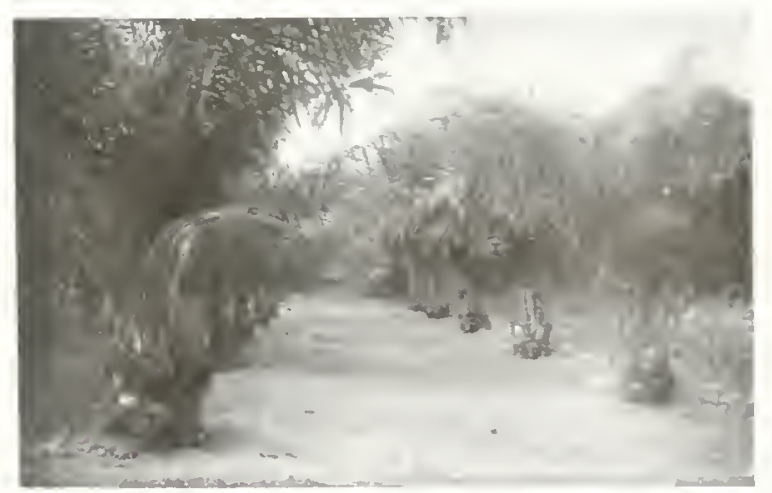

Plantaluon de palniers a huile.

Cliche Asence écononnigue $A$ O

cerne la zone sylvestre, d'un climat à peu près analogue à celui du Gaborn. On n'y constate ni fortes températures, ni variations sensibles : 20 à $30^{\circ}$ suivant les saisons, moyenne 26 ; sur la côte, $25^{\prime}$ "en forêt, 24 dans la région 
Nord ; par contre, l'humidité atmosphérique y est très grande, 90 pendant la nuit dans la région côtière, 70 à 80 dans la journée. L'hygromètre descend rarement à 65 ; il monte jusqu'à 98 , près du point de saturation (1). Cette

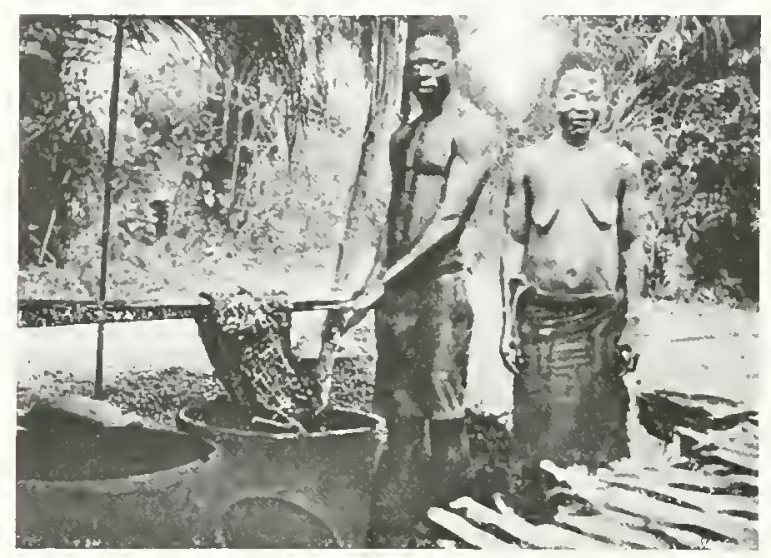

Fabrication d'huile de palme.

(Clichè Agence économique $\mathrm{A} O \mathrm{~F}$

grande humidité est pénible pour l'Européen. Son intensité décroît au fur et à mesure qu'on monte vers le Nord; à Korogho la moyenne annuelle n'est que de 68 tandis qu'elle eài de 87 à Bassam.

Les pluies sont abondantes, $2^{\mathrm{m}}, 50$ à 3 mètres sur la côte, 2 mètres à $2^{\mathrm{m}}, 50$ dans la partie moyenne de la Colonie, $1^{\mathrm{m}}, 50$ à $1^{\mathrm{m}}, 80$ dans la partie haute. Il pleut davantage dans les vallées que sur les plateaux. A Aboisso sur la Bia, à Alépé sur le Comoë, à Tiassalé sur le Bandama, il pleut pour ainsi dire toute l'année, tandis qu'à Bingerville, Abidjan et de nombreux autres points de la région côtière il y a des saisons sans pluie très caractérisées.

Tandis que dans le Nord de la Colonie il n'y a que deux saisons: hivernage, ou saison des pluies, de juin à octobre, et saison sèche, de novembre à mai, il y en a quatre bien déterminées sur la Basse-Côte:

Grande saison sèche, du 15 novembre au $l^{\text {or }}$ avril; température assez élevée. Cette saison est la plus pénible à supporter;

(1) JosepH : La Côte d'l'oire.
Grande saison des pluies, du $1^{\text {er }}$ avril au 15 juillet;

Petite saison sèche, du 15 juillet au $1^{\mathrm{er}}$ octobre, fraîche, la plus agréable:

Petite saison des pluies, du $1^{\mathrm{er}}$ octobre, au 15 novembre.

Le vent du Sud-Ouest souffle la majeure partie de l'année. Le ciel est presque toujours couvert de nuages. Il n'apparaît réellement bleu que lorsque souffle l'harmatian, ou vent du Nord, en janvier ou février; encore un brouillard épais obscurcit-il à ce moment les matinées; le soleil ne se montre que vers midi; les nuits et les matinées sont fraîches.

\section{FLORE - FAUNE}

La Flore de la Côte d'lvoire est excessivement riche, excessivement luxuriante. Nous n'avons pas l'intention d'en faire la description, M. Méniaud abordant du reste ce sujet à propos de la forêt et de son exploitation. Signalons simplement qu'on rencontre à la Côte d'lvoire la plupart des plantes des autres pays équato-

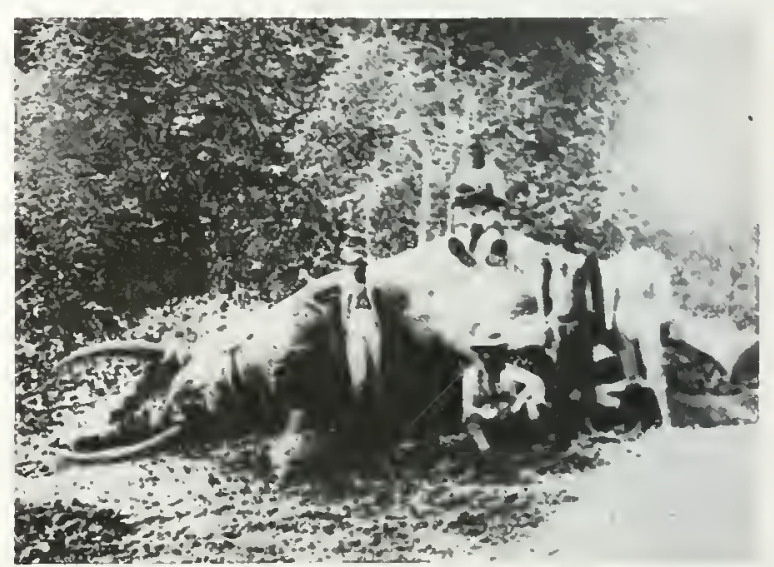

$$
\begin{aligned}
& \text { Une belle piece. } \\
& \text { Eléphant abattu par M. Burger. }
\end{aligned}
$$

riaux, et qu'on peut y tenter avec succès la culture de presque tous les produits riches dits "exotiques".

La Faune n'est pas moins bien partagée et les chasseurs les plus inexpérimentés, comme les plus adroits, peuvent s'en donner à cœur joie. 
Fauves (panthères, léopards et chats-tigres, dans la forêt, lions dans le Nord), grands pachydermes et ruminants (éléphants, hippopotames, buffles, antilopes de toutes tailles), phacochères, singes variés, gallinacées, etc..., existent partout en grand nombre.

Les animaux domestiques, à l'exception des toire aussi vaste, dont la mise en valeur exigerait une main-d'œuvre très importante. De plus. l'indigène de la Colonie travaille excessivement peu, la cueillette ou la culture des denrées nécessaires à son alimentation ne lui demandant qu un effort minime, en raison de la richesse du sol et de la force de la végétation. Il a peu de

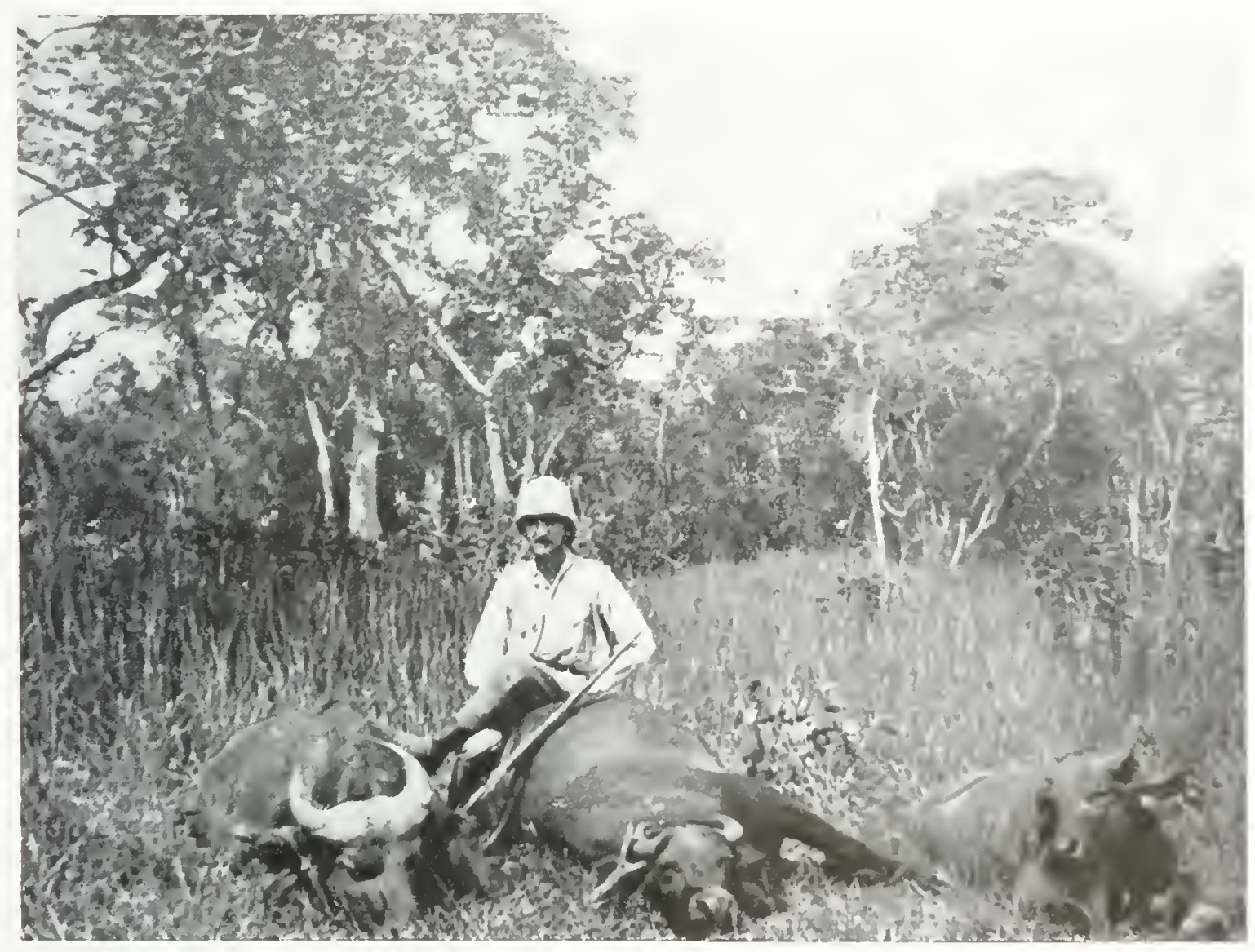

Trois buffles abattus par M. Burger.

chèvres, vivent par contre assez difficilement à la Colonie; il y a cependant une petite race de bœufs acclimatée à la forêt et dont on pourrait intensifier l'élevage. Les animaux de boucherie sont importés pour la plupart du Soudan. La volaille existe dans tous les villages. Elle est, toutefois, assez peu abondante dans la région côtière.

\section{Population - Main-d 'Euvre}

On ne compte guère à la Côte d'Ivoire que 1.400.000 habitants. C'est peu pour un terri- besoins et ne cherche pas à s'en créer. étant excessivement fruste et dédaignant toute espèce de confort. Le travail lui répugne naturellement et. à l'exception de quelques populations côtières qui fournissent des marins, et des Abey's qui sont d'excellents bûcherons. on ne trouve pas toujours commodément des rolontaires pour les services publics. la colonisation et les exploitations forestières. Il est bon cependant de signaler que. gràce à l"intervention de l'Administration. qui agit par persuasion auprès des Chefs, colons et exploitants forestiers 


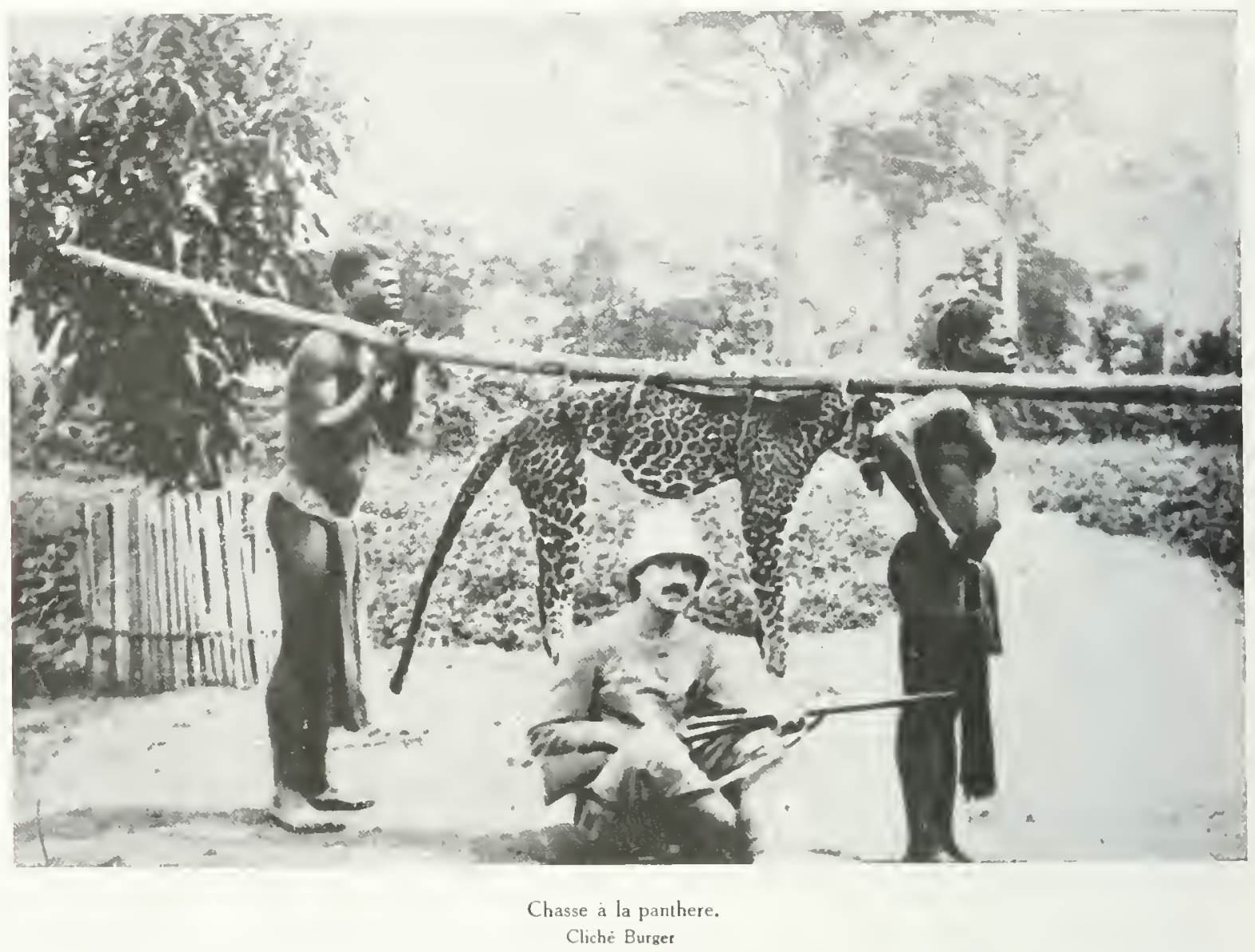

n'ont presque jamais manqué, jusqu'à présent, de la main-d'oeuvre qui leur était nécessaire.

Le Gouvernement local ne néglige rien, d'autre part, pour attirer et fixer des travailleurs étrangers. Le prolongement du chemin de fer vers le Nord rendra de gros services à cet égard en facilitant les relations entre les régions peuplées de la Boucle du Niger et la Basse-Côte.

\section{COMMERCE}

Le Commerce général de la Colonie était de 35 millions en 1912 et en 1913. Les premières années de guerre ne furent pas favorables aux affaires; plusieurs maisons durent suspendre tout ou partie de leurs opérations; la majeure partie des chantiers forestiers durent être arrêtés. Peu à peu, cependant, sous l'influence de la hausse qui se manifesta progressivement sur tous les produits, le commerce reprit son activité; des chargements importants d'acajou furent demandés d'Angleterre, puis d'Amérique pour la fabrication d'hélices d'avions. Dès 1918, le mouvement commercial enregistré à l'entrée et à la sortie éiait remonté à 29 millions. Il se développa rapidement (en 1919, le chiffre dépassait 50 millions) et en 1920 il atteignait un total de 115 millions.

Nous donnons ci-après le relevé des principaux produits exportés en 1919 et en 1920:

\begin{tabular}{|c|c|c|c|}
\hline & & En 1919 & $\begin{array}{c}\text { En } 1920 \\
-\end{array}$ \\
\hline les de boufs & kg. & 67.458 & 84.918 \\
\hline brutes de moutons. & - & 16.378 & 10.304 \\
\hline Defenses d'eléptrant. & - & 2.961 & 2.894 \\
\hline Graines de ricin & - & 341.585 & 272.410 \\
\hline Amandes de palmistes. & - & 16.115 .196 & 10.966 .297 \\
\hline Cafè.... . & - & 110.128 & 17.298 \\
\hline Cacao (en feves) & - & 959.487 & 1.036 .222 \\
\hline Pimentset poivre de Guinee & - & 44.862 & 71.393 \\
\hline Huile de pa'me. . & - & 12.593 .973 & 8.655 .193 \\
\hline Glu. & - & 53.620 & 18401 \\
\hline Coprah & - & 41.184 & 992 \\
\hline Beurre de karite. & - & 10.873 & 8.143 \\
\hline Caoutchouc brut. . . & - & 76.310 & 91.020 \\
\hline
\end{tabular}


En 1919

En 1920

$\begin{array}{lrrr}\text { Noix de kola . . . . kg. } & 17.954 & 17.199 \\ \text { Bois d'ebenisterie: Acajou } & \text { mc. } & 34.345 & 50.193 \\ \text { Bois autres durs. . . . } & - & 964 & 15.520 \\ \text { Coton. . . . . . . } & \mathrm{kg} & 336.263 & 250.210 \\ \text { Kapock. . . . . . . } & - & 77.208 & 8.072 \\ \text { Raphia . . . . . . } & - & 10.200 & 2.119 \\ \text { Nattes indigenes (piece) . } & - & 4918 & 11\end{array}$

Nous ne connaissons pas encore les chiffres de 1921. Il est à prévoir qu'ils sont sensiblement inférieurs à ceux de l'année précédente, en raison de la crise dont ont souffert la plupart des produits exporiés de la Colonie, corps gras et bois notamment.

Les produits divers, huile de palme, palmistes, cacao, etc., sont expédiés pour un peu plus de moitié sur la France, le complément sur l'Angleterre, la Hollande et l'Allemagne. Les acajous vont, pour les 35 aux Etats-Unis et en Angleterre, les 25 restani étant dirigés en grande partie sur la France, où sont également expédiés les bois autres dont on a commencé l'exploitation. L'Allemagne, qui était un des gros clients de la Colonie avant la guerre, fait de nouveau quelques achats.

En ce qui concerne les importations, la majeure partie des articles manufacturés provient d'Angleterre et d'Amérique. C'est le cas, surtout pour les tissus, les tabacs, métaux, matériaux de construction, outillage de toute sorte. Notre industrie nationale, si éprouvée par la guerre, n'est pas encore suffisamment représentée. Elle reconquiert peu à peu cependant la place qu'elle a perdue. Le régime douanier de la Colonie ne la favorise pas, malheureusement. et il lui est difficile de lutter, pour certains articles, contre la concurrence étrangère.

\section{Centres comerciaux}

Les principaux centres commerciaux sont : Grand-Bassam, au débouché du Comoč eî de la lagune Ebrié, et Abidjan, terminus du chemin de fer sur la lagune. Après viennent : $D_{a}$ bou, sur la lagune ćgalcment, Grand-Lahou,
Sassandra, Tabou, Assinie, points d'embarquement sur l'Océan, Aboisso, sur la rivière Bia à proximité de la lagune Aby, Agboville, Dimbokro et enfin Bouaké, sur lã ligne du chemin de fer.

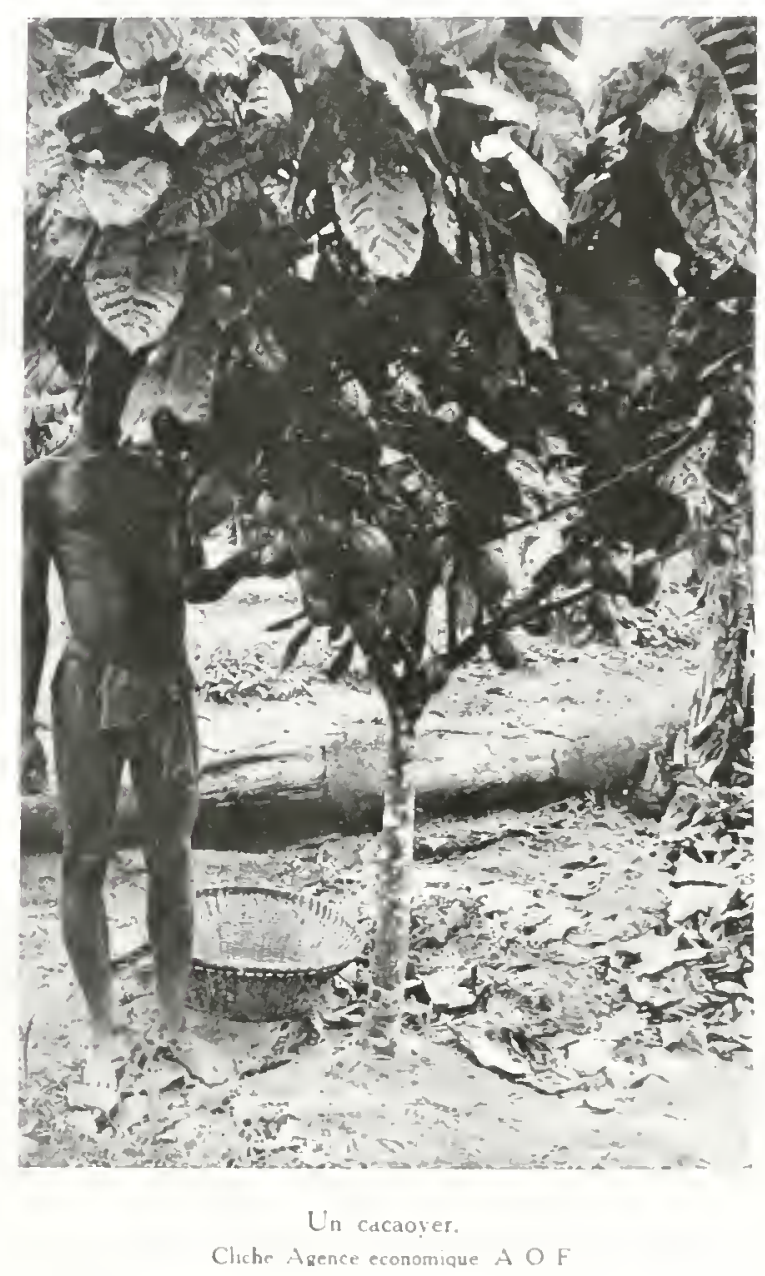

La plupart des maisons représentées ont leur siège en France, à Bordeaux ou Marseille. On en trouvera la liste plus loin.

\section{RÉCHIE, DOUANIER}

La Côte d'Troirc fait partie. ainsi que le Dahomey, des territoires soumis à la convention internationale de 1898. Aux termes de cette convention. les sujets britanniques, les produits importés d'Angleterre ou de ses colonies. jouissent de tous les avantages accordés. dans les deux colonies prćcitées, aux sujets et aux pro- 


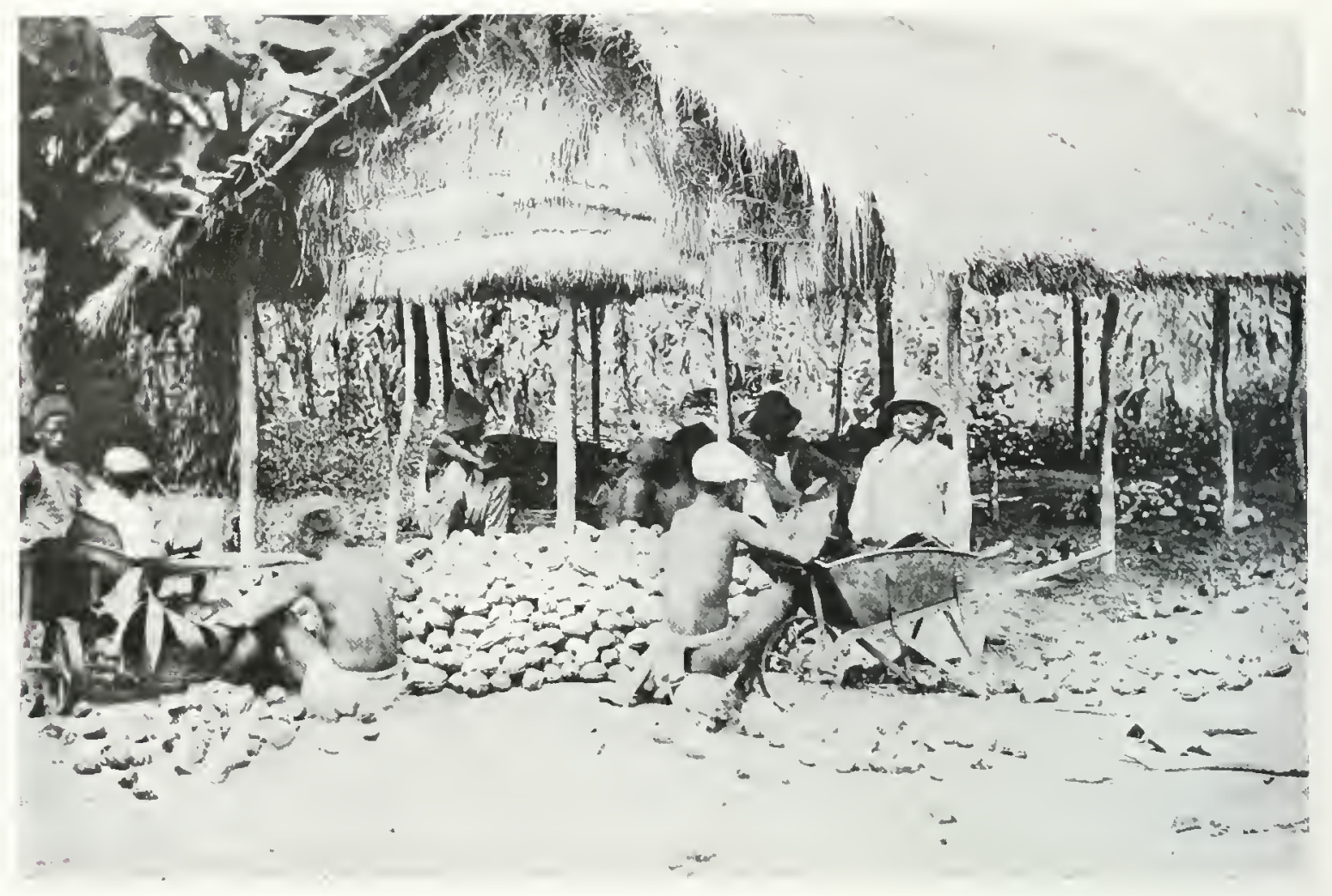

Préparation du cacao.

Cliché de l'A gence économique A. O F.

duits français. A la suite de cette convention, les pays ayant des traités de commerce avec la France et jouissant chez nous du traitement de la nation la plus favorisée, demandèrent le bénéfice des avantages réservés à l'Angleterre dans nos possessions de la Côte d'Afrique. On en vint bientôt à ne plus faire aucune distinction entre les différentes nations importatrices et le régime douanıer établi pour la Côte d'lvoire et le Dahomey est depuis longtemps applicable uniformément à toutes les provenances (1). Or,

(1) Droits d'importation fixés à $10 \%$ de la valeur des marchandises importées, majorée de $25 \%$. Certains artıcles sont toutefois soumis à des tarifs spécifiques; les principaux sont :

Les sucres qui doivent payer $40 \mathrm{fr}$. par $100 \mathrm{kgs}$; les tabacs en feuilles, $600 \mathrm{fr}$. par $100 \mathrm{kgs}$; les tabacs fabriqués, de 1.650 à $2.400 \mathrm{fr}$. par $100 \mathrm{kgs}$, suivant qualités; les alcools de consommation, de 280 à $1.000 \mathrm{fr}$. par hectol. d'alcool, suivant qualités et degrés d'alcool: les tissus de coton, de 120 à $580 \mathrm{fr}$. les $100 \mathrm{kgs}$; les tissus de laine, de 480 à $900 \mathrm{fr}$. les $100 \mathrm{kgs}$; les tissus de soie, de 275 à $1.240 \mathrm{fr}$. les $100 \mathrm{kgs}$. (Tarifs 2" semestre 1921.) si ce fait pouvait se justifier à la rigueur avant la guerre, il ne se justifie plus maintenant et il serait grand temps de songer à modifier sur ce point le décret du 14 avril 1905 qui reste la charte douanière de l'Afrique Occidentale Française. Qu'on continue à traiter sur le même pied que les produits français les produits venant d'Angleterre, de Belgique, d'Italie, des EtatsUnis, de toutes les nations qui ont coopéré à la victoire de 1918, c'est tout à fait normal, mais il ne paraît plus nécessaire d'accorder ce même traitement aux articles allemands, par exemple, qui, à la faveur d'un change extrêmement bas, sont fabriqués à un prix bien meilleur marché que chez nous et nos alliés, et envahissent rapidement tous les marchés des pays qui ne prennent pas de mesures pour se défendre contre cet envahissement.

La question n'a pu échapper au Gouvernement Général de l'Afrique Occidentale Française. Il est donc à présumer que des modifi- 
cations au régime douanier de la Côte d'Ivoire et du Dahomey interviendront assez prochainement.

\section{INDUSTRIE}

A la Côte d'lvoire, comme dans les autres Colonies de l'Afrique Occidentale, l'indigène,

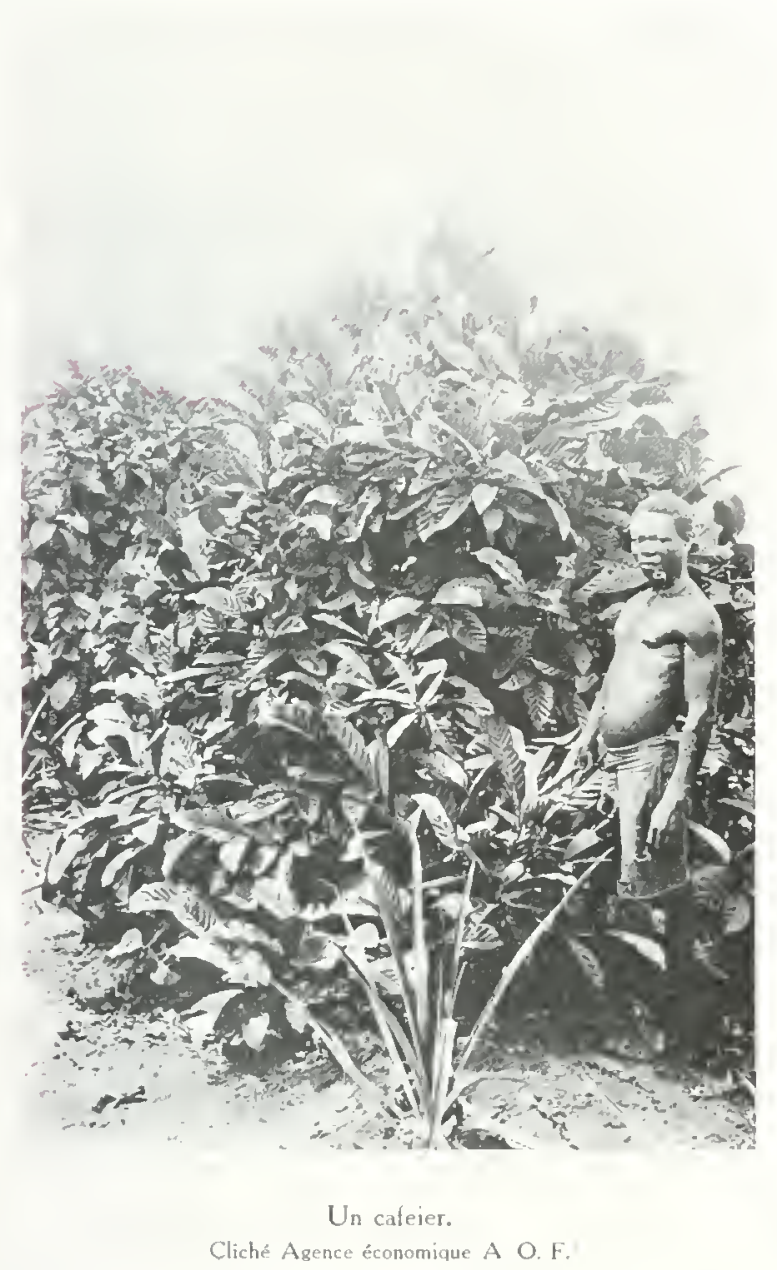

s il arrive à produire tout ce qui est nécessaire à son alimentation, à fabriquer tout ce dont il ne peut se passer, continue à employer des procédés extrêmement rudimentaires; qu il travaille le fer, le bois ou le cuir, file ou tisse du coton, teigne des tissus, fasse du savon, de Thuile. l'article ou produit obtenu esi toujours plus ou moins grossier; le noir n'apporte à la préparation ou à la confection aucun soin particulier, ne cherche pas à faire mieux que ses devanciers.
Si quelques tissus ou cuirs ourragés présentent des dessins assez harmonieux ou originaux, on peut tenir pour certain que ces dessins ne sont pas d'origine locale, mais ont été apportés par des voyageurs ou des tribus imrnigrés de l'Orient ou de l'Afrique du Nord. On se borne à les reproduire de génération en génération, sans y apporter la moindre modification.

Dans la région côtière, l'industrie principale est celle de la préparation de l'huile de palme. Nous verrons, quand sera traitée cette question. combien les procédés de fabrication demanderaient à être perfectionnés.

La pêche et le séchage du poisson, la fabrıcation des pirogues sont également parmi les industries à signaler.

L’industrie européenne est représentée par quelques huileries mécaniques et par trois importantes scieries qui débitent les bois employés sur place ou exportés dans les colonies voisines. C'est dans ces deux branches qu'elle est appelée à se développer.

\section{Colonisation \\ RÉGME DES CONCESSIONS}

La colonisation européenne à la Cóte d'lroire, si elle est sortie de la période d'études et de tâtonnements. est encore bier peu développée. II faut dire que la pacification du pays ne date réellement que de 1911-1912. Si, auparavant, les Européens avaient déjà commencé à s installer un peu partout à la Colonie, ce n'était néanmoins quaux alentours immédiats des postes militaires ou administratifs, sous la protection des troupes ou des gardes indigènes. Nais on n'osait pas trop pénétrer dans l"intérieur et surtout demander, pour les défricher et les mettre en valeur ensuite, des superficies importantes de terres, les indigènes restant plus ou moins hostiles à l'octroi de concessions rurales, hostiles également aux Européens qui se seraient aventurés à occuper des terres dont ils revendiquaient la proprićté, tout en reconnaissant cependant qu ils ne les utilisaient pas et ne les avaient jamais utilisées. 


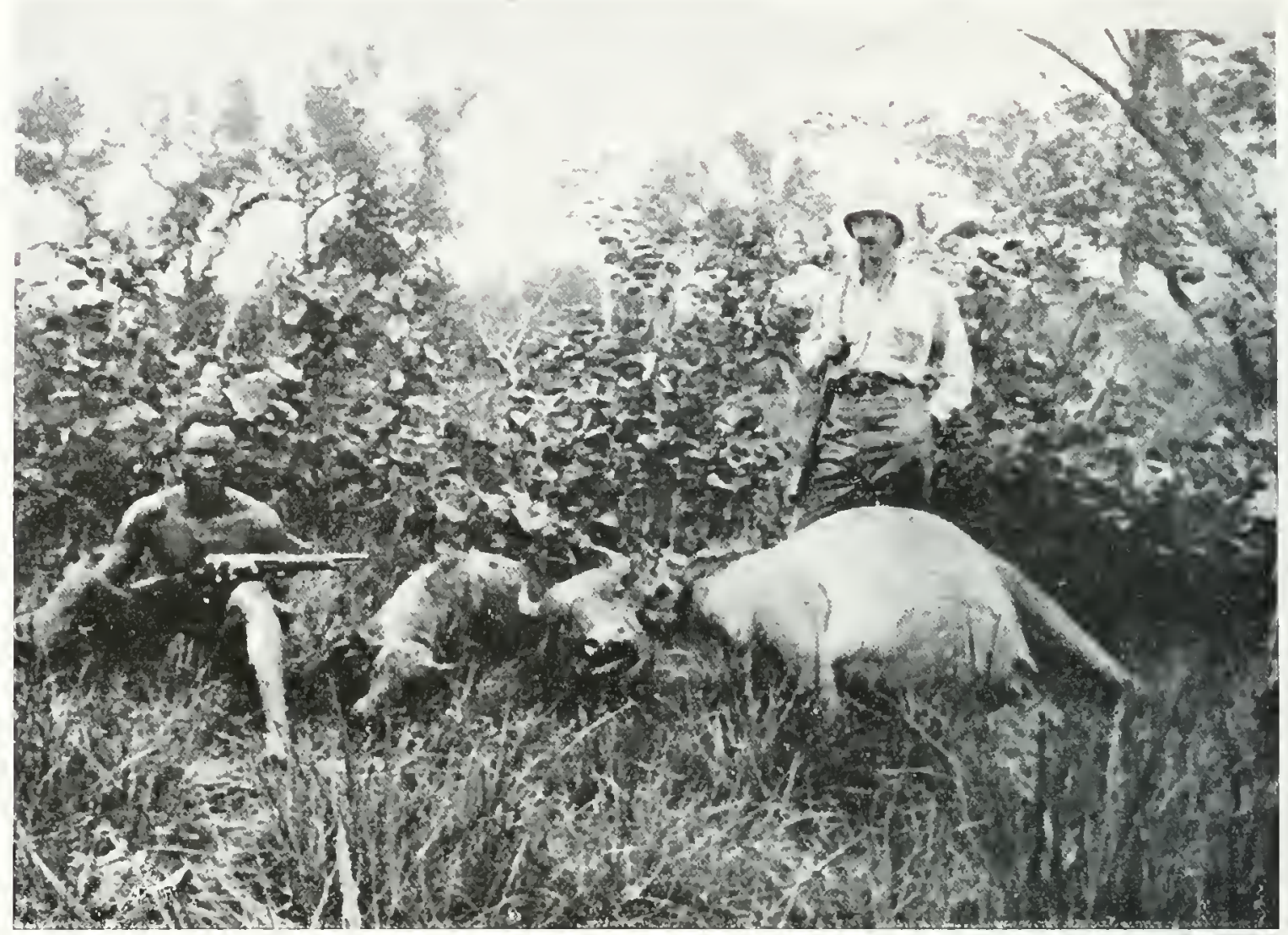

Chasse au buffe.

Cliché Burger)

Puis, la guerre est venue et, de 1914 à 1919. le développement de la colonisation fut paralysé, un peu en raison de l"incertitude de l'avenir et, surtout, du nombre trop restreint d'Européens restés à la Colonie.

Malgré cela, on compte aujourd'hui à la Côte d'Ivoire une quinzaine d'exploitations agricoles intéressantes, autant par les leçons qui peuvent en être tirées, que par les résultats déjà obtenus. 400 hectares plantés de palmiers à huile; 200 plantés de cocotiers, 800 à 1.000 de cacaoyers, 100 de caféiers, le tout de très belle venue et commençant à entrer en rappori. Plus nombreuses sont les exploitations amorcées en 1919 et 1920. D’ici cinq ou six ans, si le mouvement entrepris ne subit pas d'arrêi, la colonisation européenne occupera déjà à la Colonie une place importante.

Du côté indigène, une centaine au moins de petites plantations individuelles de cacaoyers, de 5 à 20 hectares. Le nombre et la superficie de ces plantations s'accroissent rapidement.

Les collectivités, elles, exploitent, mais sans aucun soin, un cinquième à peine des immenses palmeraies qui sont à leur disposition. Elles en retirent l'huile et les palmistes qui sont vendus au commerce. Elles cultivent, en outre, mais uniquement pour leurs besoins, manioc, ignames, maïs, bananes; dans le Nord, riz et sorgho, ainsi qu'un peu de coton dont elles emploient la majeure partie pour leur usage personnel.

L'Administration locale ne ménage, aux uns comme aux autres, ni ses conseils, ni ses encouragemenis. Les terres vacantes, les palmeraies inexploitées sont si nombreuses qu'il lui est possible, sans porter aucunement préjudice aux autochtones, de donner des concessions à quiconque veut exercer son activité dans le domaine 
cultural. Les concessions, jusqu’à 200 hectares, sont accordées par le Gouverneur; au-dessus de 200 hectares et jusqu'à concurrence de 2.000 hectares, par le Gouvernement général. En principe, elles sont attribuées à titre provisoire et moyennant une légère redevance ( $0 \mathrm{fr} .50$ par hectare) pour une durée de cinq ans. Un cahier des charges est établi et, à l'expiration du délai spécifié ou avant, si les conditions de mise en valeur imposées ont été remplies, elles peuvent être obtenues à titre défnitif. Le délai de cinq ans peut être, d'autre part, prorogé dans certains cas.

Nous aurons à revenir plus longuement sur toutes ces questions lorsque seront examinées, dans un autre fascicule, les conditions et l'avenir des différentes cultures entreprises ou pouvant l'être dans la Colonie, ainsi que la préparation ou le traitement industriel dont les produits obtenus doivent être l'objet avant leur exportation, pour assurer aux planteurs le maximum d'avantages.

\section{Moyens de COMMUNiCATIONS}

Fleuves et Lagunes. - Nous avons vu que la Côte d'Ivoire, pour un pays où il pleut énormément, est assez peu favorisée au point de vue des cours d'eau, voies naturelles de communication. A côté des fleuves et rivières, il existe toutefois un réseau de lagunes, qui se développe parallèlement à la Côte et qui est extrêmement intéressant.

Ces lagunes, qui ont des fonds de 2 et 3 mètres, soni séparées de la mer par un cordon littoral dont la largeur varie de un à six kilomètres. Elles forment trois systèmes différents :

1" La lagune Aby et la lagune Tendo. qui couvrent une superficie de 296 kilomètres

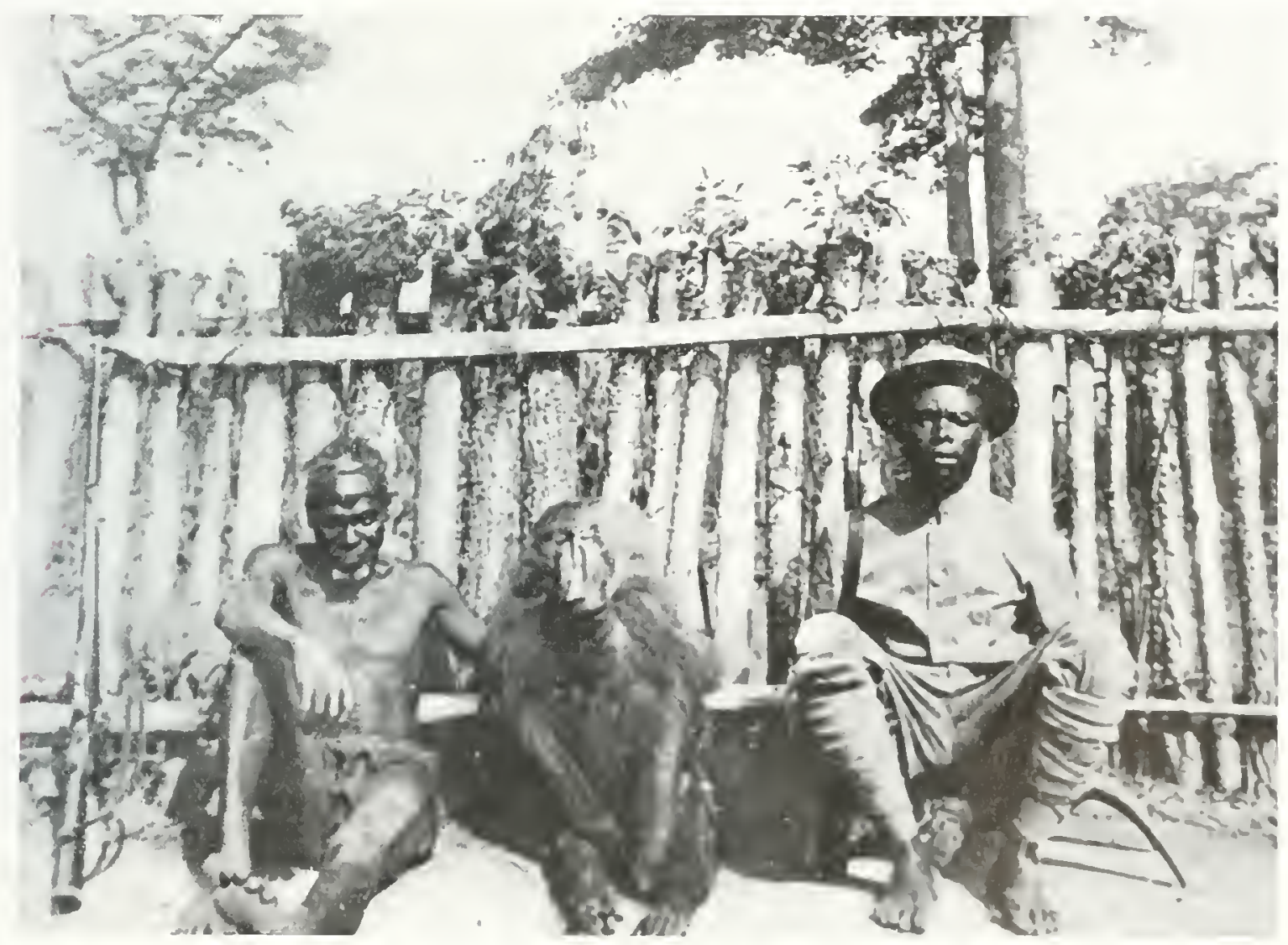

Mandville en capturite

Cliché Burger 
carrés et desservent toute la région située à l’Est et au Nord d'Assinie;

$2^{\circ}$ La lagune Ebrié, qui, avec la lagune M'Potou, couvre une surface de 570 kilomètres

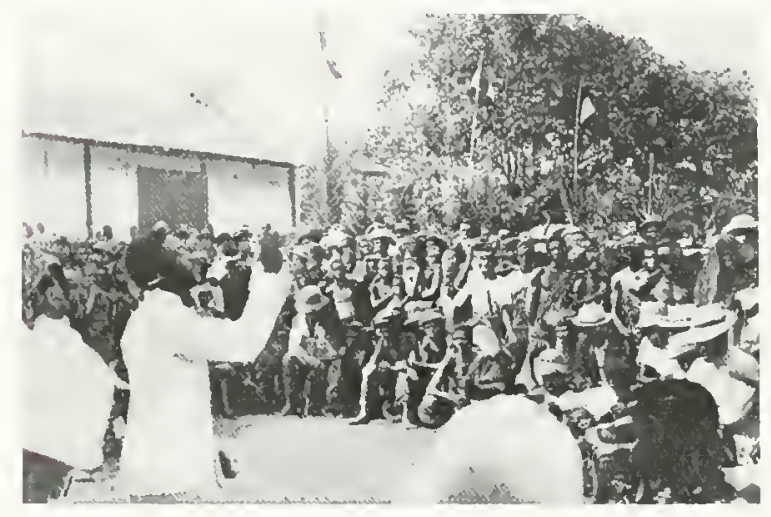

Un palabre à Dabou.

Clıché Agence économıque 'A O.F.

carrés et s'étend sur 120 kilomètres à l'Ouest de Bassam ;

$3^{\circ}$ La lagune de Lahou, couvrant 395 kilomètres carrés, longue de 40 kilomètres.

Les deux derniers systèmes ont certainement communiqué autrefois. Des études faites à ce sujet, sur l'initiative de M. le Gouverneur Angoulvant, permirent d'entreprendre la construction d'un canal de jonction. Les travaux commencèrent fin 1917. Ils furent poussés très activement. On perça tout d'abord un canal de six mètres, profondeur $0^{\mathrm{m}}, 60$, pour le passage de pirogues pouvant transporter voyageurs et marchandises, canal achevé dès 1918. Les travaux du canal définitif (20 mètres en gueule, 8 mètres au plafond, $2^{\mathrm{m}}, 40$ de profondeur), effectués au moyen d'une drague, atteignent actuellement le kilomètre 13. 1ls seront achevés au cours de l'année 1922. La longueur totale du canal sera de plus de 16 kilomètres.

La lagune de Lahou communique, d'autre part, à l'Ouest avec la petite lagune de Fresco, par un canal naturel, plus ou moins obstrué à l'époque des basses eaux.

Chemins de fer. - Une seule voie ferréo a été construite dans la Colonie. Elle part
d'Abidjan, sur la lagune Ebrié, à 40 kilomètres à l'Ouest de Bassam, se dirige vers le NordOuest et atteint Bouaké, à 316 kilomètres du point de départ. Son prolongement vers le Nord d été repris au début de 1921.

D'après le programme de grands travaux établi par M. Albert Sarraut, cette voie ferrée sera poussée jusqu'au Mossi, par Bobo-Dioulasso. Un embranchement desservira Sikasso et Koutiala. Terminée, elle mettra une partie du Soudan et de la Haute Volta, hinterlands naturels de la Côte d'lvoire, dans la dépendance économique de cette dernière Colonie.

Un autre embranchement, d'intérêt plus local, partira de Dimbokro, dans la direction de Bouafflé et de Daloa, pour desservir la partie centrale et ouest de la Colonie. Il s'arrêtera momentanément au fleuve Bandama et sera prolongé par un réseau de routes automobilisables.

Routes. - Celles-ci se divisent en deux catégories, routes provisoires automobilisables en saison sèche, et routes défnitives automobilisables à peu près toute l'année. Ces dernières sont faites déjà sur plus de 300 kilomètres :

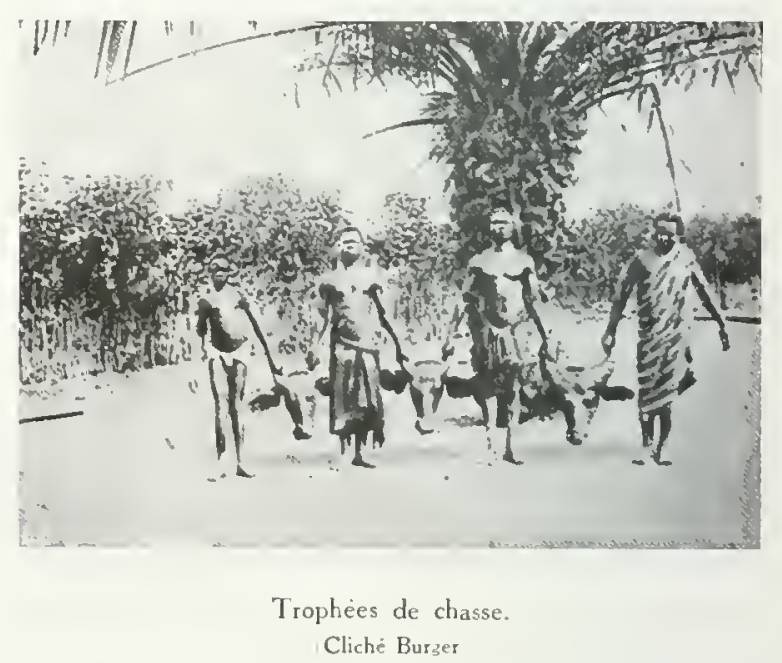

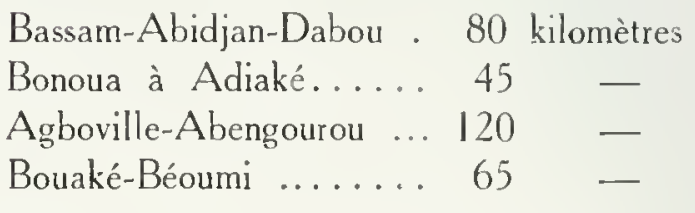

Les autres, considérablement améliorées depuis quelques années, comprennent plus de 
1.000 kilomètres de longueur. Rendons, ici, hommage à $M$. le Gouverneur Antonetti, les efforts faits sous son administration pour développer les transports par automobiles et faciliter les déplacements dans la Colonie, étant vraiment remarquables.

Ports. - La Colonie ne possède pas de ports à proprement parler. Tabou, Sassandra, Grand-Lahou, Grand-Bassam, Assinie, ne sont que des points d'un littoral presque rectiligne en face desquels s'arrêtent les bateaux qui veulent laisser ou prendre des chargements. tion, à côté de l'ancien, d'un wharf neuf, plus puissamment outillé. La construction en sera achevée au début de 1922. Un troisième wharf est, en outre, prévu à Vridi, en face d'Abidjan, pour répondre au développement du trafic.

Quelque progrès qu'ils constituent sur les moyens d'embarquement dont on dispose dans les autres centres commerciaux de la côte, ces wharfs sont encore des instruments bien précaires. Leur emploi est coûteux; il est irrégulier. Viennent plusieurs bateaux à la fois en rade, ils ne peuvent répondre aux nécessités du trafic;

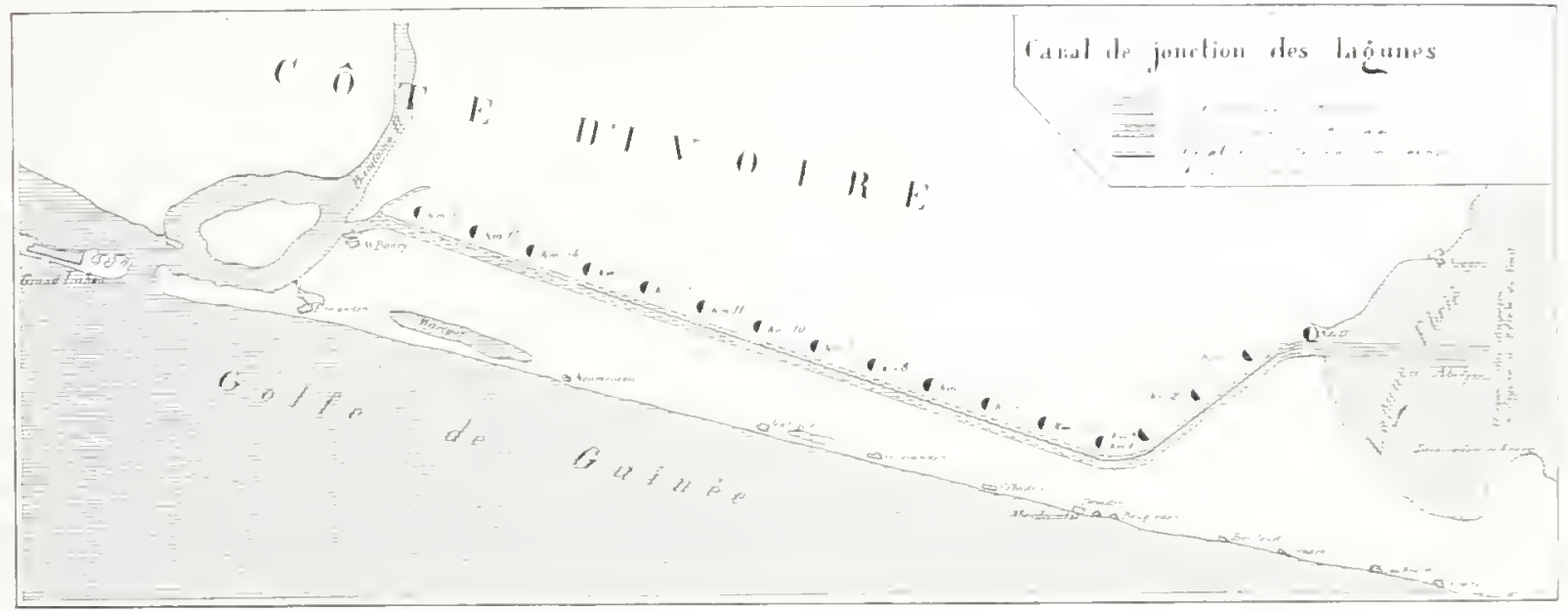

Encore, ces bateaux sont-ils obligés de se tenir à une certaine distance de la côte, en raison de la faiblesse des fonds et surtout en raison de l'action de la "barre " qui rend impossible la station des navires dans le voisinage immédiat du littoral.

Grand-Bassam, au débouché du Comoë et de la lagune Ebrié, en relation avec l'intérieur par le chemin de fer qui aboutit à Abidjan, est de beaucoup, le plus important de ces points d'embarquement. Un wharf y fut construit dès 1918 pour faciliter les opérations de chargement et de déchargement. Ce wharf a rendu de très gros services. Malgré les réparations dont il a été l'objet, il est aujourd'hui bien fatigué; il est devenu, de plus, insuffisant et l'Administration locale a dû envisager la construc- par gros temps, ils doivent suspendre complètement leurs opérations. Il en résulte des surestaries, des tarif́s de fret très élevés, qui paralysent, momentanément, le déreloppement économique de la Colonie. Aussi a-t-on, depuis déjà longtemps, étudié la possibilité de créer. à Bassam ou dans le voisinage, un véritable port en eau calme. Deux projets ont été retenus, qui permettraient, l'un et l'autre, de faire entrer les bateaux maritimes dans la lagune Ebrié. Le coût élevé de leur réalisation ( 80 à 100 millions de francs de travaux) n'en permet pas, malheureusement, l'exécution actuellement et il est à prévoir quaucune décision ne sera prise arant que le trafic ait atteint un déreloppement déjà important, dépassant la capacité de rendement des wharfs qui vont être construits. 
On a bien envisagé un moyen intermédiaire qui consisterait à faire passer, par l'embouchure du Comoë, des bateaux allèges à très faible tirant d'eau faisant la navette entre la lagune et les bateaux ancrés dans la rade foraine de Bassam et doublant le trafic effectué au moyen des wharfs. La faiblesse et l'irrégularité des tonds dans le chenal, les déplacements assez ment. Le trafic se développe néanmoins, beaucoup moins rapidement, certes, que si la Colonie disposait d'un port. Certaines industries, dont on peut prévoir le succès. ne pourront être créées, en effet, que lorsque le pays sera pourvu d'un outillage plus moderne. Dès maintenant, cependant, avec le concours des wharfs, il est possible de développer les cultures industrielles,

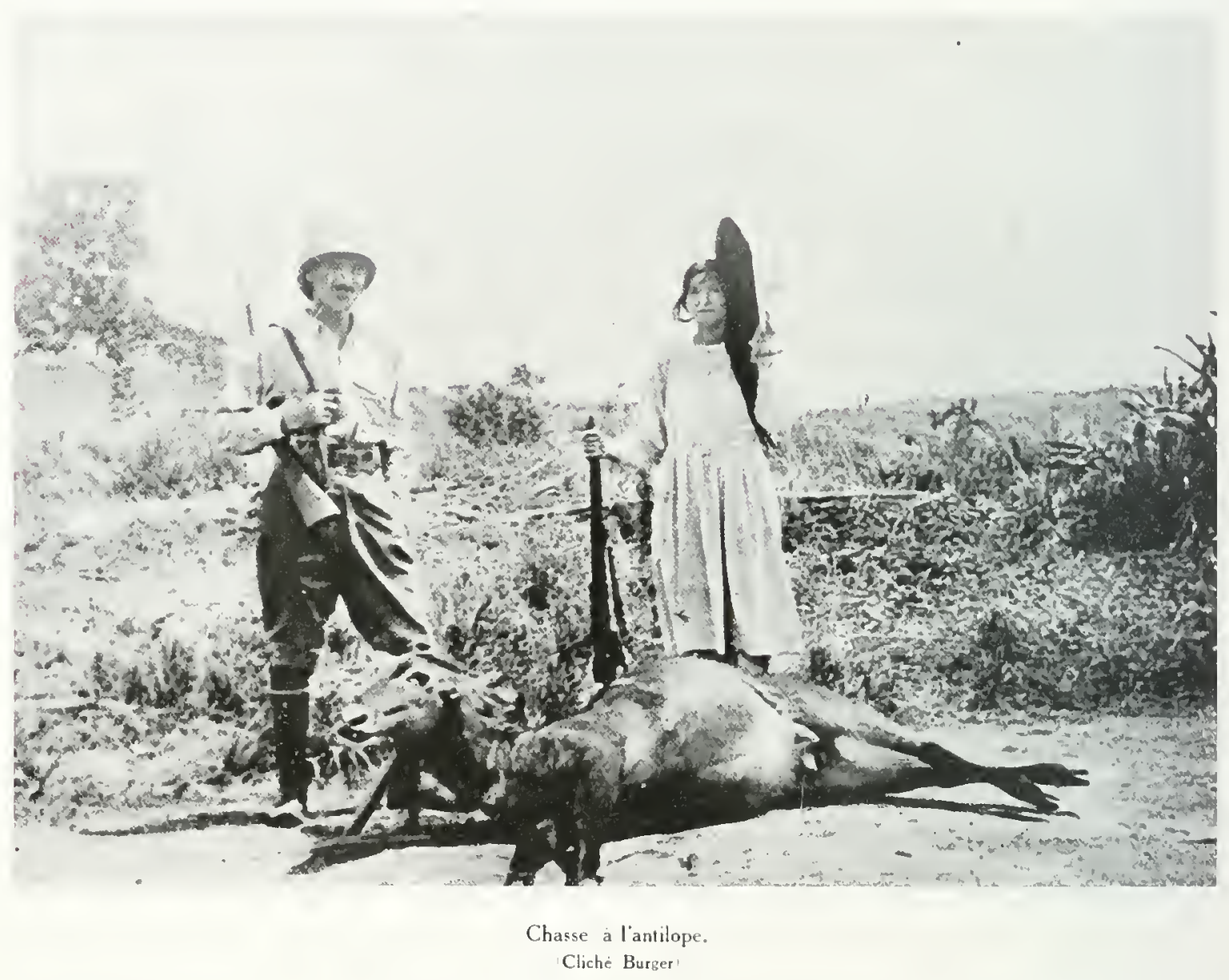

fréquents de ce dernier, ses sinuosités, les déferlements de la barre à l'endroit où, précisément, les fonds sont le plus faible et le plus irrégulier, laissent présumer le passage de ces bateauxallèges comme assez aléatoire. Des études complémentaires sont, en tout cas, nécessaires avant qu'il puisse être permis de se prononcer sur la valeur du projet.

La Colonie souffre donc, provisoirement, de l'insuffisance, du manque de perfectionnement des moyens d'embarquement et de débarque- comme celles du palmier à huile, du cocotier, du cacauyer, du caféier, du bananier dont les produits peuvent trouver un écoulement facile et rémunérateur, d'exploiter plus intensivement la forêt.

\section{RELATIONS AVEC L'EUROPE}

Ne terminons pas ce court aperçu sans donner quelques indications sur les relations existant entre la Colonie et la Métropole.

La Côte d'Ivoire n'est pas favorisée, depuis 
la guerre, au point de vue des communications maritimes. Avant 1914, en effet, les bateaux de la Cie Belge du Congo, touchaient régu-

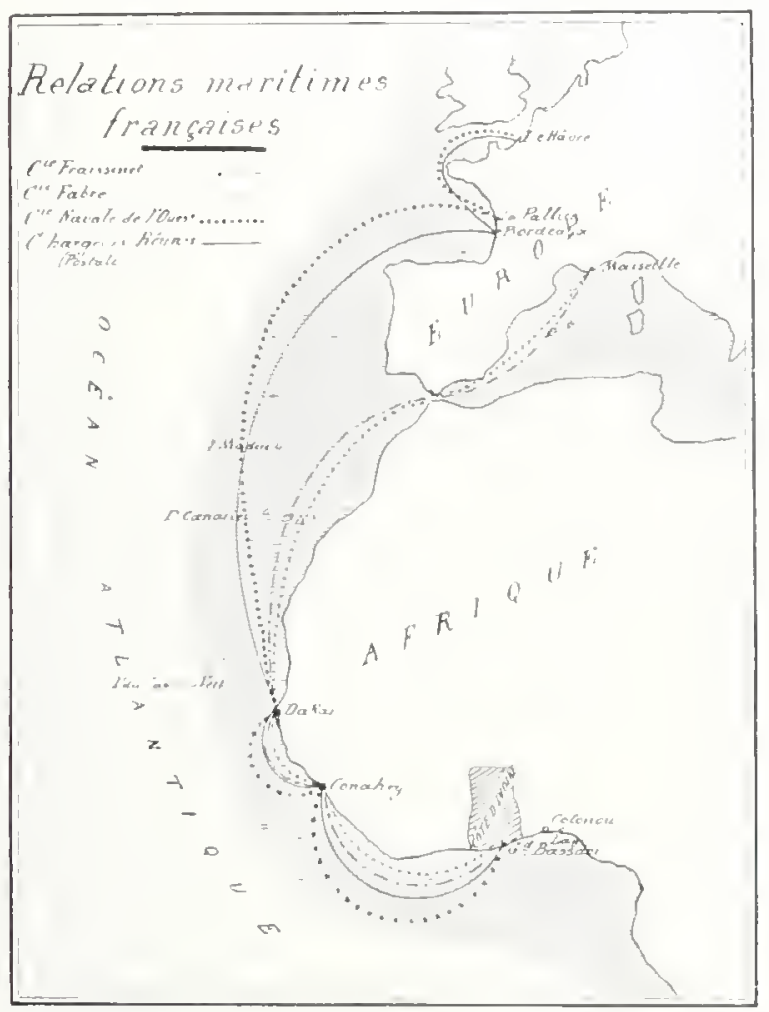

lièrement Grand-Bassam et faisaient escale à l'aller et au retour à La Pallice. Les Woërmann, qui faisaient escale à Boulogne, et les navires de la Cie anglaise Elder Dempster passaient trois fois par mois à Grand-Bassam. Tous ces bateaux concurrençaient assez sérieusement les lignes françaises. Actuellement, il n'en est plus de même: les Woërmann ne recommencent à se montrer que de temps à autre et on comprend qu'ils ne fassent plus escale en France: les Elder Dempster ne touchent plus régulièrement à Bassam, à cause du mauvais fonctionnement du vieux wharf, et les bateaux de la $\mathrm{Cie}$ Belge du Congo filent directement de Konakry sur Matadi ou inversement.

La Cie française des Chargeurs Réunis, avec un départ de Bordeaux tous les vingt-cinq jours. reste donc momentanément sans concurrent sérieux. Le voyage dure quinze jours, avec escales à Dakar. Konakry et 'Tabou ; il coûte relativement très cher, pour des paquebols qui. en dehors de l" Asie ", sont assez peu confortables.

La Cie Fraissinet a, sur la même ligne. quelques bateaux mixtes qui partent de Marseille, bateaux qui ne prenrent qu'un nombre très limité de passagers. Le roỵage MarseilleBassam dure dix-sept à dix-huit jours, avec escale dans la plupart des ports français et étrangers de la Côte Occidentale d'Afrique.

\section{LISTE DES PRINCIPALES MAISONS DE. COMNERCE, INDUSTRIES, BANQUES, PLANTATIONS}

INSTALLEES A LA COTE DIVOIRE

\section{Maisons de Commerce}

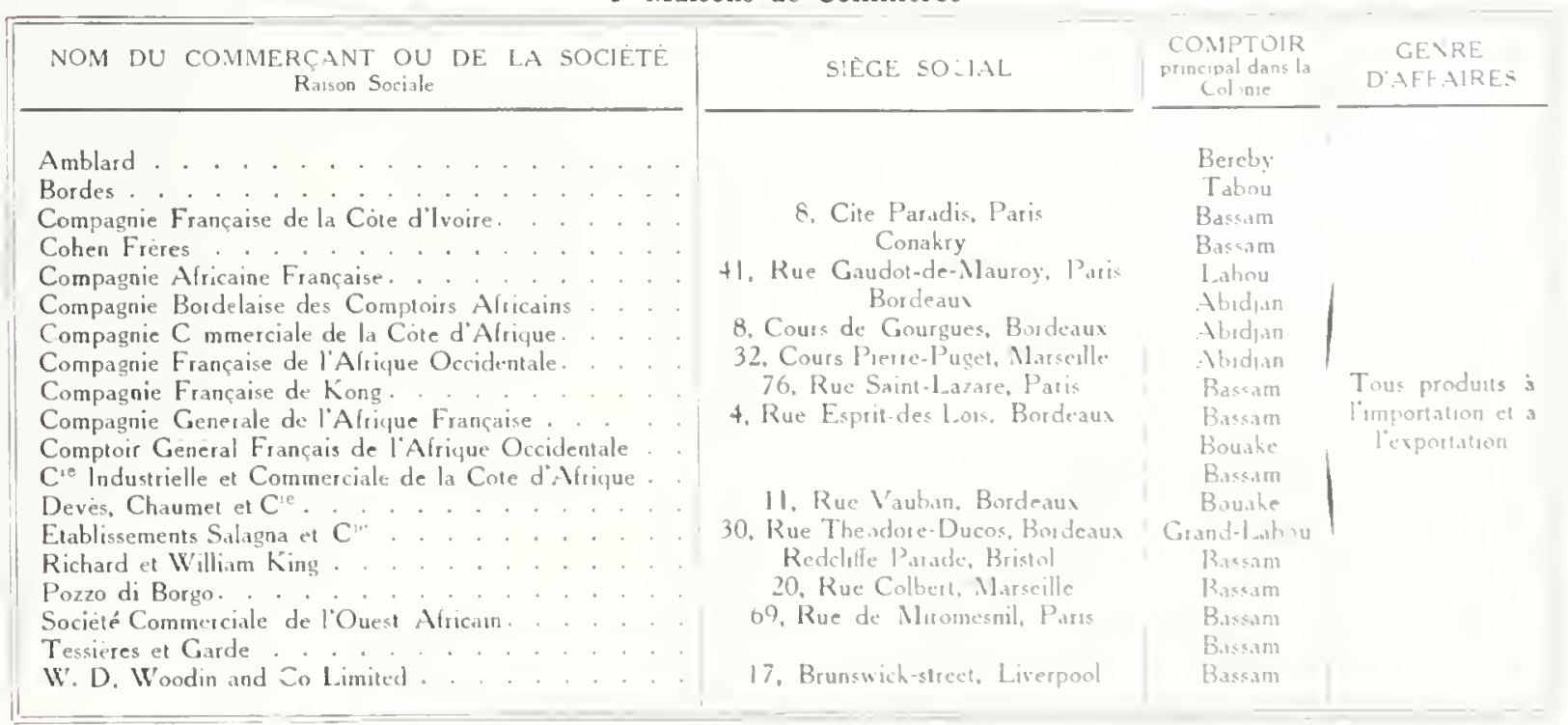


2 Principaux Exploitants et Exportateurs de Bois de la Cöte d'Ivoire

$$
\text { EXILITA STS }
$$

Récur di Rusum-itridan

compiname Fineolute de l'Atrugur.

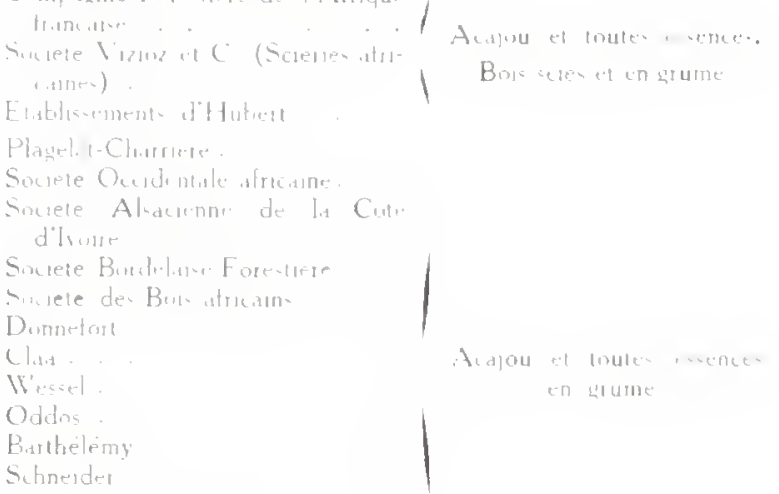

S.hnerder

semel

Lamber-Cisumont

Akit Edousd

l'irrivet

$$
\text { Revinn di.tisinic }
$$

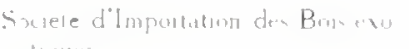

lipues

viluelas.

Poizal

$$
\text { Recunn de Luhoui }
$$

$V_{i, 1} l_{k}$ freme

Concele For -41.r. da Bandama

Matian

Siemantz
Siner
Binnet
Cecuy

Pecuyes

Pieric

$$
\text { Regrom de sussundras }
$$

Sincle lndurtulle du Bor

$$
\text { ACHEIEI RS DE BOIS }
$$

Mitguet, à Buram

Mither, a Bersam

Gutzwiller, a Bisum.

Sociple de Bois alacinn

surete lizur el

Hamition and $C$

Wiondin and $C$

1 Actor

Acalou el loutes essences.

F IPURTATELRS

Acapou pout Fiance el Angleters

Acilou pour tmerique.

I Acajou et diverspour France

I et autres pats d'Europe.

Aingu pour Angleterre.
3 Plantations principales de Cacao, Café, Cocotiers

Comprome Colonsule de la Bua a dicime.

Blachon, a Bimemill.

Borde., a Tabru.

Comprame de Kiong, a Bianam.

Clement. a B.m.n

King, a Tiansle.

Lemourt, a A - nome:

>echrt. a MPBatr.

\section{Banques}

Banalu= de l'Alrifue Occidentale. a Bussam Toutes operations bancalles

Banyue de l'Alrupue Equatarale Toutes operations lidncaires.

\section{Transitaires-Commissionnaires}

Ramul Chensrau, a Bossam.

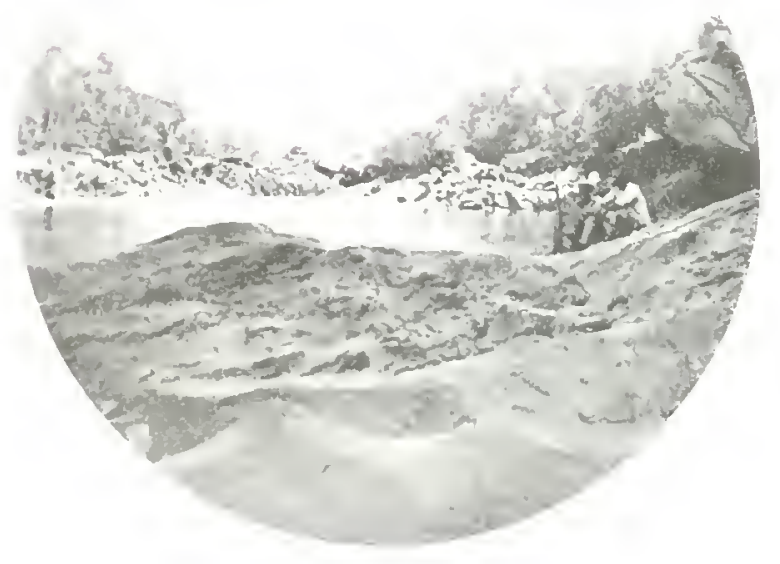

P.y.ay du Cinmon

Clintie Rugan 


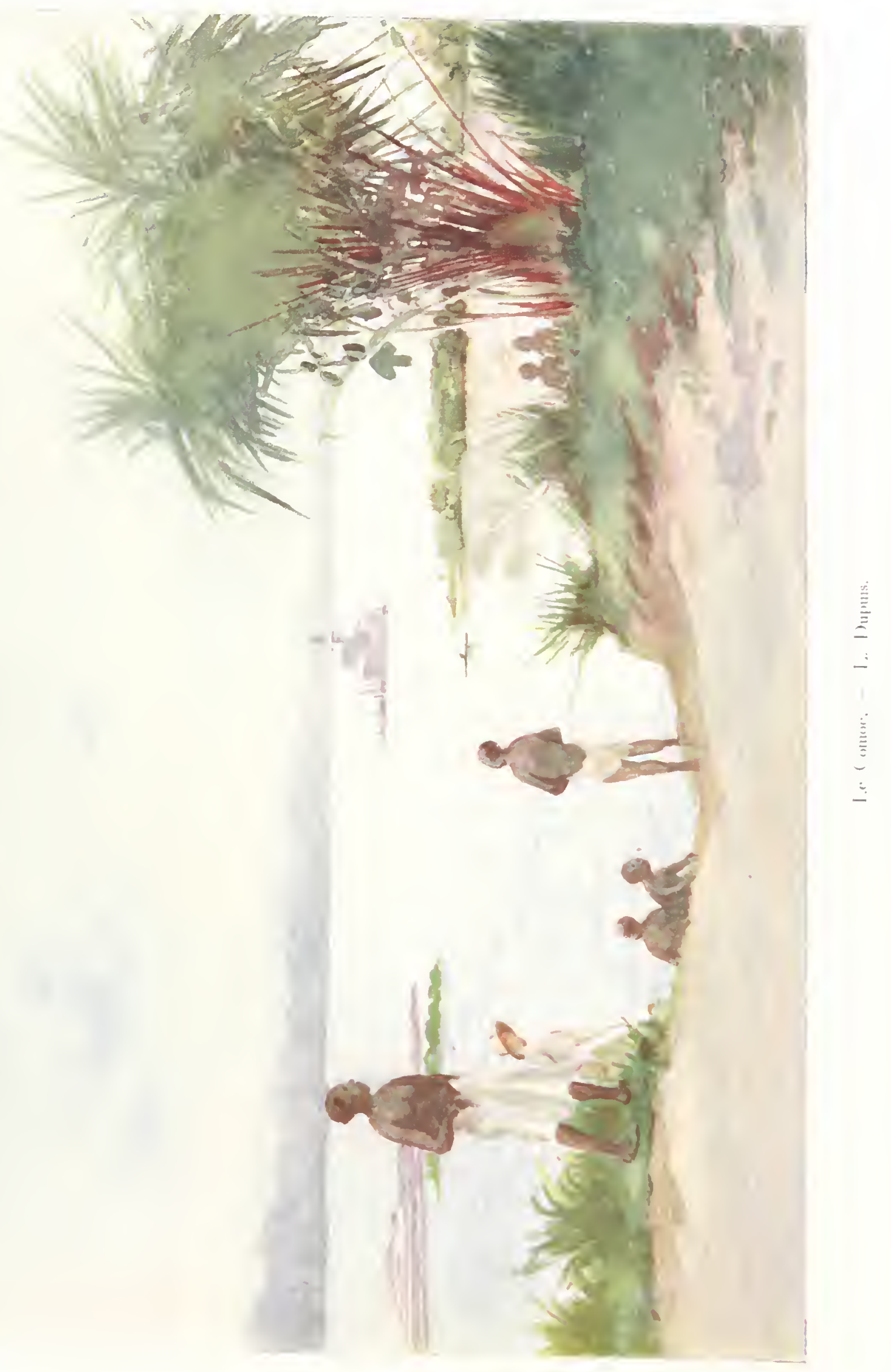



A la mémoire de ma chère disparue, $A$ mon frère Jacques.

J. $M$.

\section{TITRE PREMIER}

\section{La Forêt \\ Conditions de son exploitation}

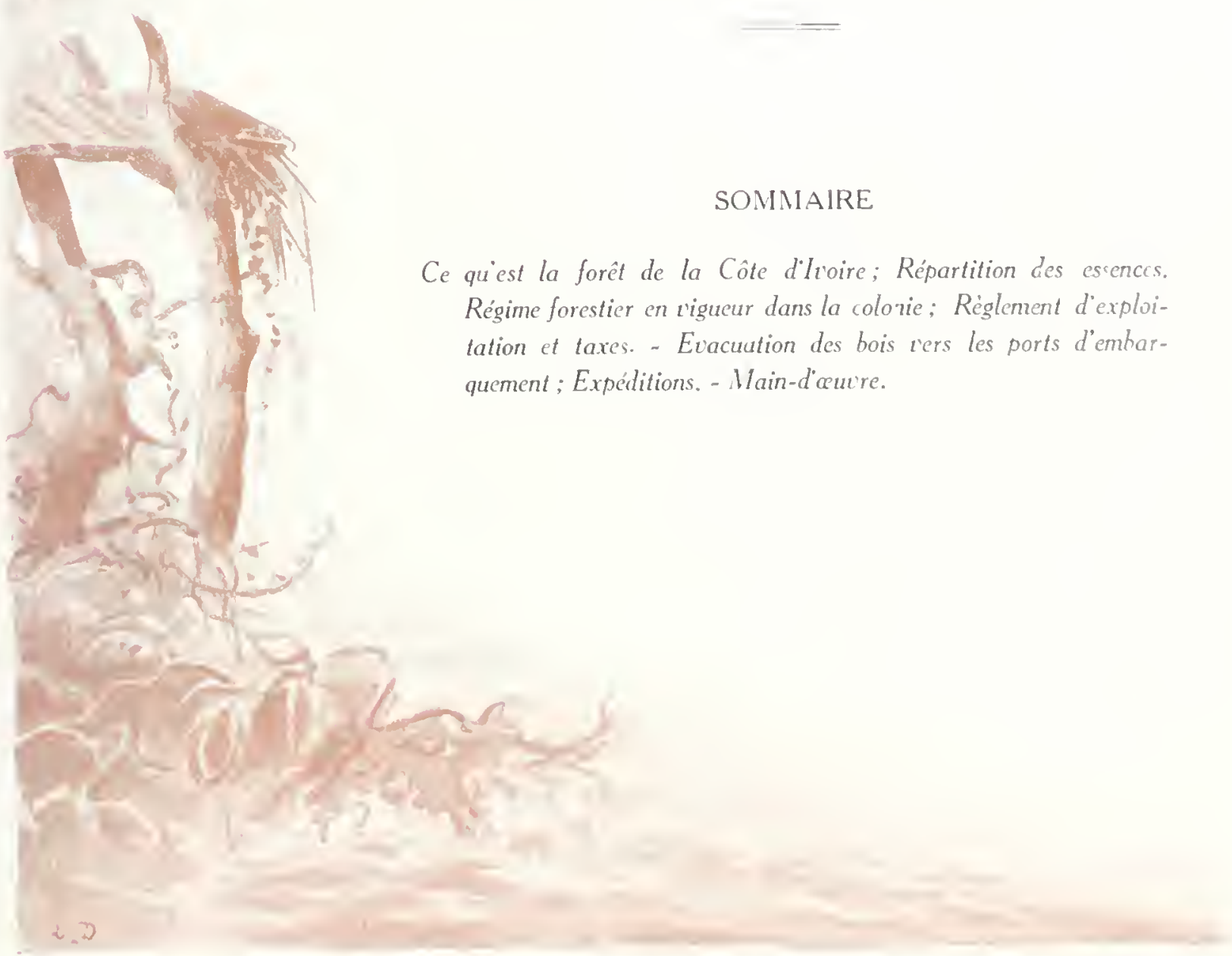




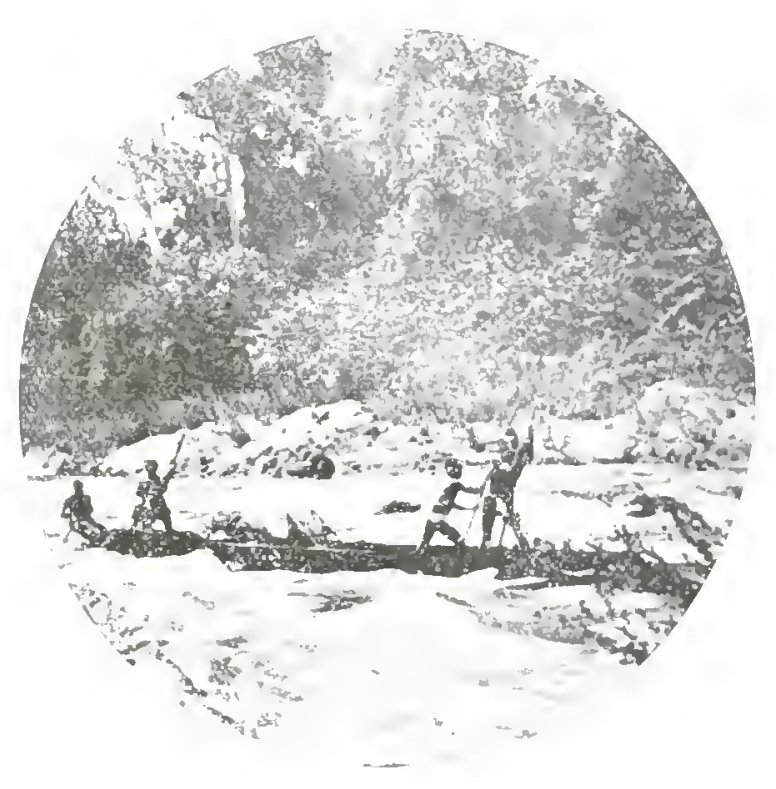




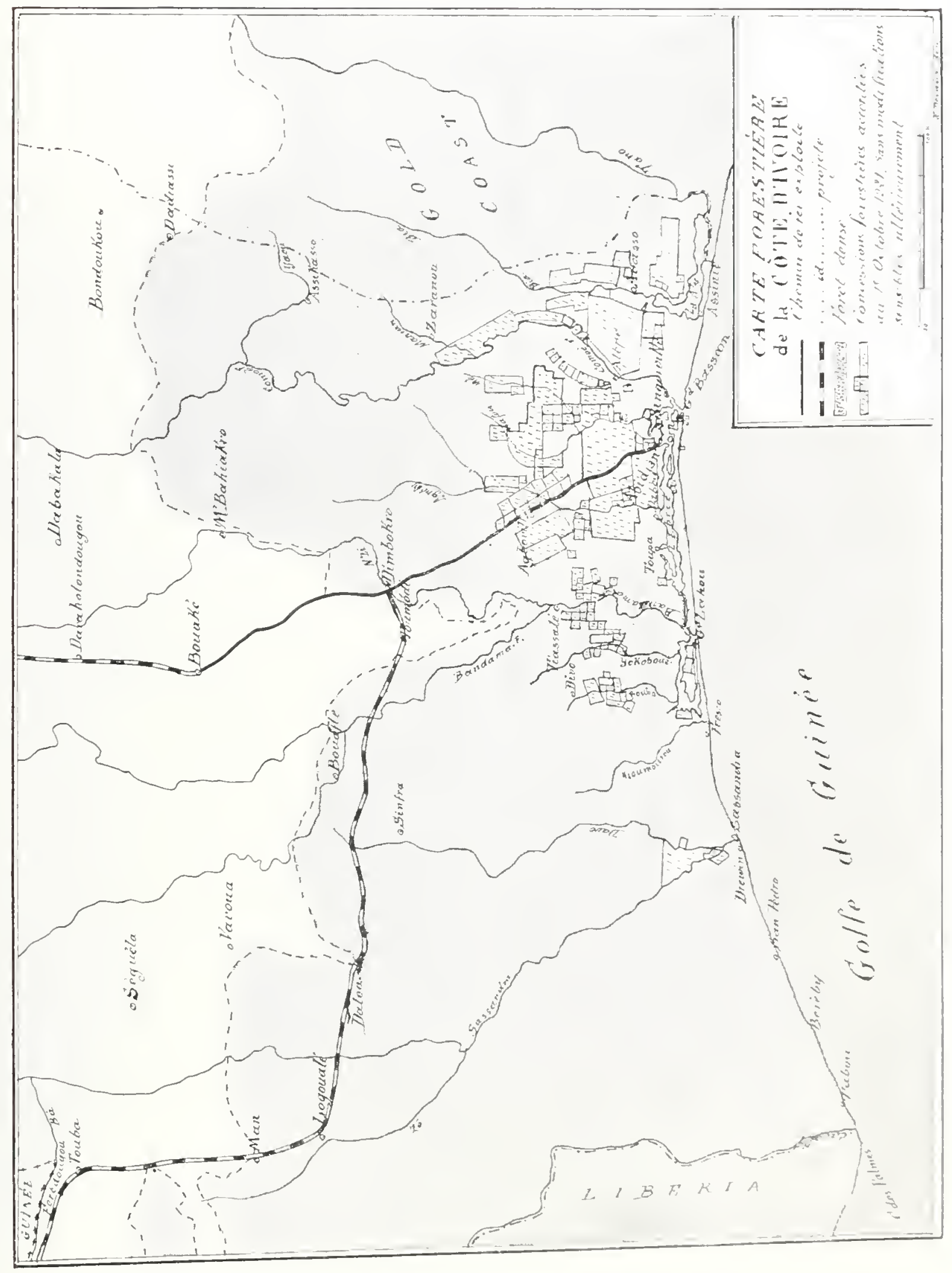





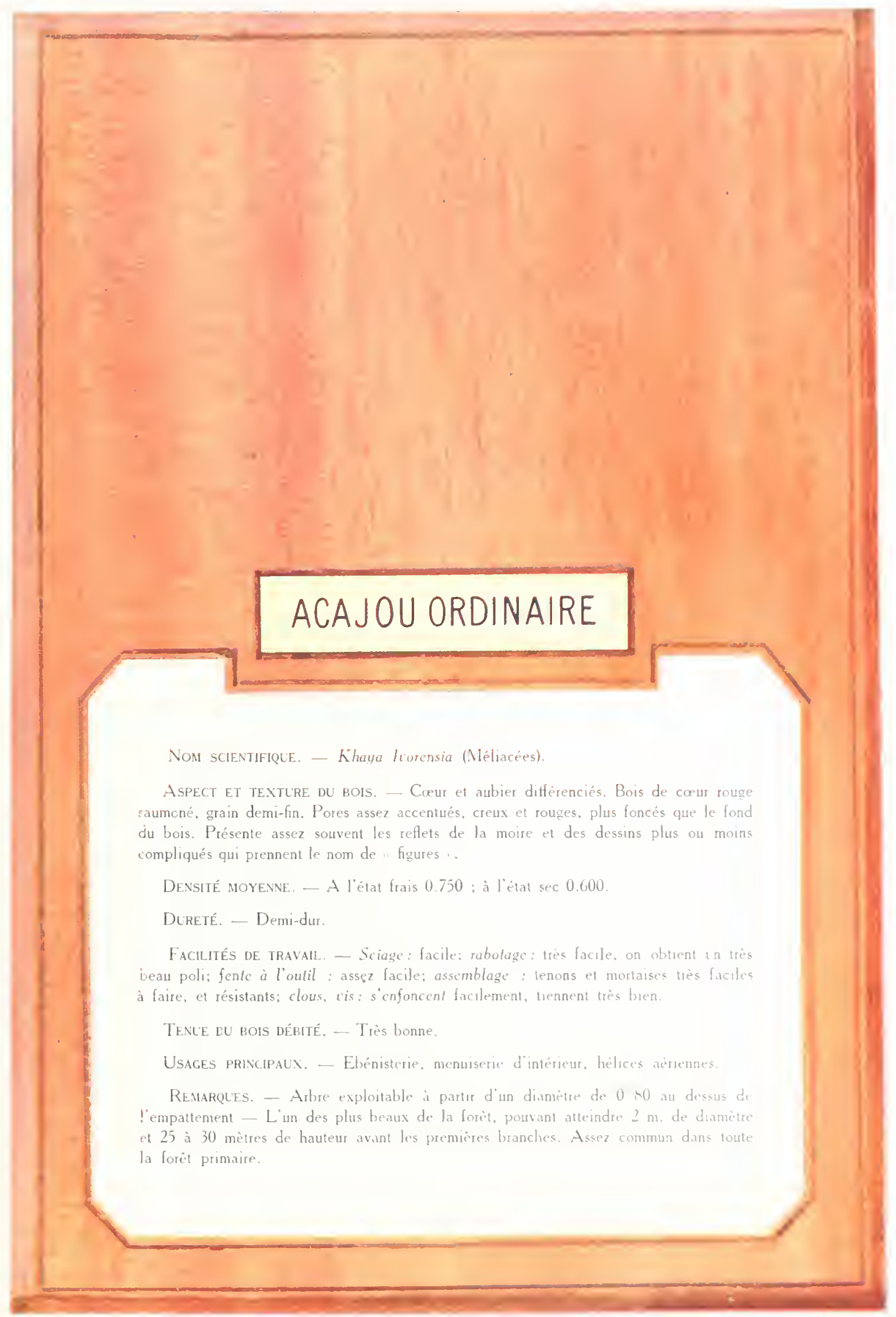



TITRE PREMIER

\section{La Forêt. - Conditions de son exploitation}

\section{CE Qu'est la forêt de la Cote d'I voIRE}

La forêt de la Côte d'lvoire offre un spectacle extraordinairement imposant. Quiconque l'a parcourue plus ou moins conserve l'impression d'avoir entrevu un monde nouveau, où la lumière pénètre à peine, où les dimensions, la majesté des grands arbres contrastent avec l'amas, souvent inextricable, de lianes qui rampent sur le sol, grimpent aux troncs ou retombent des branches. "Cette forêt, dit M. G. Joseph, garde, malgré les voies commerciales qui la traversent. son aspect mystérieux. Elle demeure comme un immense écran à la vision. Elle rebute. Une singulière appréhension saisii lorsqu' on s'engage sous sa voûte " (I).

La force de la végétation y est vraiment prodigieuse. Ce n'est pas tant la grande hauteur des arbres qui frappe - en France même nous avons des peupliers ou des sapins qui sont peutêtre plus élevés encore - c'est le caractère spécial de ces arbres dont le tronc lisse et cylindrique, sauf à la base même, sans nouds, atteint 20, 25 et même 30 mètres avant les premières branches. Les racines rampanies, pour la plupart des essences, émergent en bourrelets. constituant autour du tronc une sćrie de contreforts qui assurent à l'arbre une assiette solide, mais rendant l'abatage au ras du sol assez difficile.

(1) La Còte d'lioire, par G. JosepH.
Ceite forêt s'étend sur 12 millions d'hectares environ. La zone sylvestre couvre en effet plus du tiers de la superficie totale de la Colonie; elle prend naissance près de la côte et ne s'arrête, aux extrémités Est et Ouest, qu à 300 kilomètres dans l'intérieuĩ. Au centre, les savanes du $\mathrm{N}^{\prime} \mathrm{Z}$ i s'avancent comme un coin dans la forêt dense. A l'endroit le plus resserré, la distance qui sépare ces savanes de la côte est encore de 150 kilomètres. Cette immense étendue de forêt ne forme pour ainsi dire qu' un bloc au travers duquel les petits villages indigènes, les espaces défrichés pour les cultures, ne constituent que de minuscules éclaircies.

Qu'un terrain utilisé par les indigènes pour leurs plantations soit abandonıé pour un autre. la végétation reparait immédiatement et avec une puissance extraordinaire. C'est tout d'abord un vaste amas de broussailles. de ronces. duquel émergent quelques palmiers génćralement poussés là par hasard. (1) et qu'a respectés l'indigène, et des tiges d'essences diverses parmi les plus vivaces. La course à la lumière commence: toutes ces tiges, trop serrées. s'allongent démesurément et arec une rapidité inouïe pour essayer de dominer leurs rolsines. Les essences spongieuses luttent dans cette course avec succès. mais la rapidité de leur croissance est quelque-

(1) La graine a pu itre apportée par un viseau ou un animal qui s'est nourri du truit. 


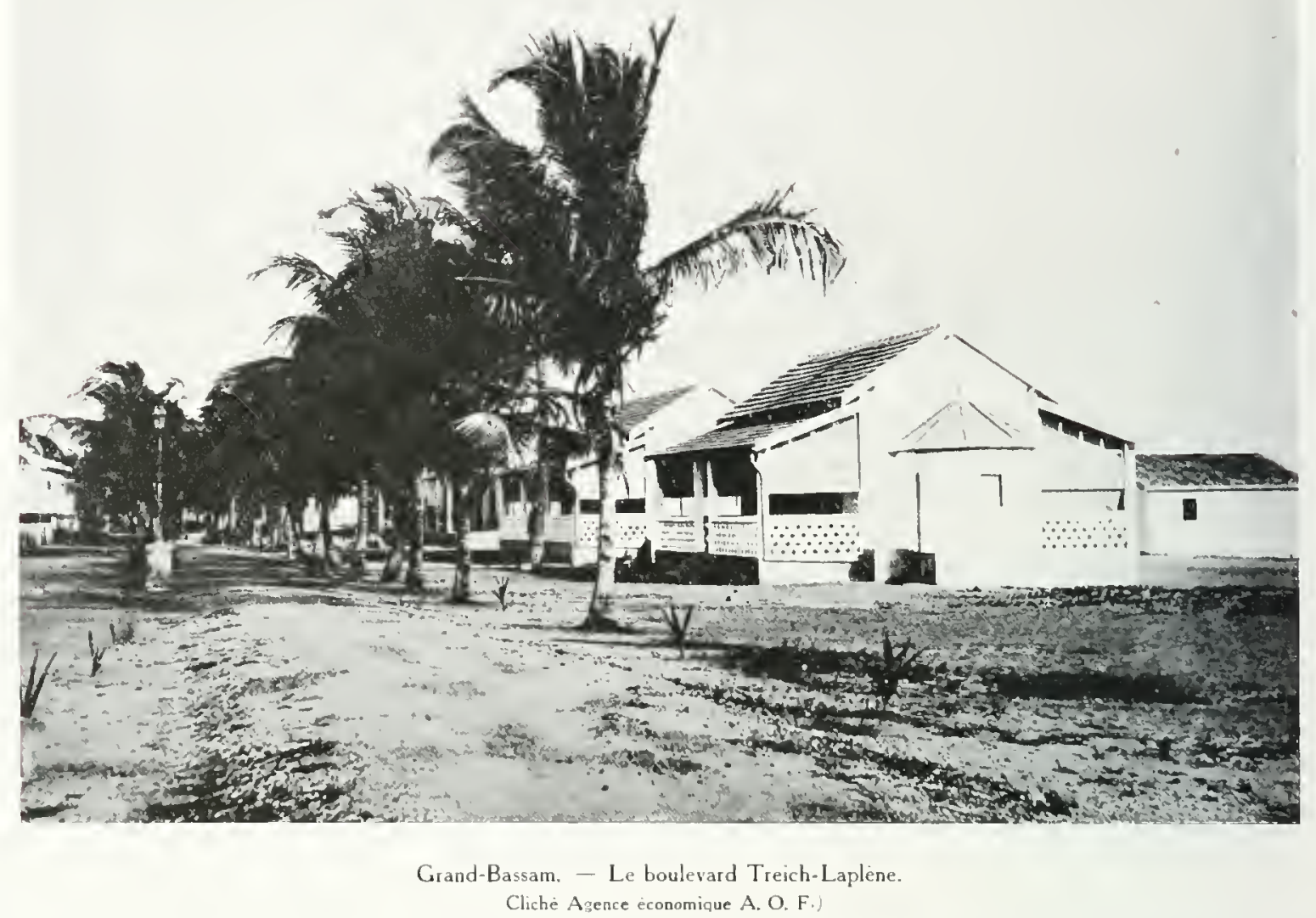

fois un facteur de leur perte et les essences dures, plus résistantes, qui ont pu vivre sous leur couvert, reprennent le dessus quand le grand arbre qui les a dominées a été cassé ou arraché par le vent ou est arrivé à la limite de sa croissance, de sa vie (l). Quant aux palmiers qui ont réussi à ne pas périr sous la végétation environnante, on comprend qu'ils soient étiolés, allongés démesurément, peu aptes à produire des fruits.

La forêt renferme un très grand nombre d'essences. Le docteur Aug. Chevalier a pu en faire un inventaire méthodique. Les seuls arbres qui atteignent de grandes dimensions comprennent plus de 350 variétés. Plus récemment encore, la Mission des Bois Coloniaux, dirigée

(1) La forêt appauvrie par des défrichements ou des coupes non rationnelles se régénèrerait donc d'elle-même à la longue; c'est un point important qu'i] est difficile d'élucider, mais qui parait plausible. par l'Inspecteur des Eaux et Forêts Bertin, a étudié les peuplements d'une quarantaine d'essences utilisables par l'industrie. C'est de ces dernières essences que nous nous occuperons, encore que bon nombre d'enire elles ne puissent être exploitées immédiatement, faute de pouvoir être traitées sur place ou de pouvoir être transportées dans des conditions suffisamment avantageuses.

Les peuplements sont denses partout, mais ils manquent totalement d'homogénéité. La densité de telle essence détermiriée varie en effet d'une région à l'autre, suivant la nature et la composition du sol, le degré d'humidité, etc..., elle peut représenter jusqu'à 25 ou $30 \%$ (1) des peuplements dans certaines régions, tandis qu'en d'autres elle sera rare ou même introu-

(1) Il y a même des peuplements, près des lagunes, qui sont composés pour $80 \%$ de "Bahia " ou de $40 \%$ de "Fou " ou de "Rikio ". 
vable. Encore peut-elle, quand on la trouve, présenter, par l'écorce ou le bois lui-même, des différences d'aspect assez caractérisées qui font se demander si l'on ne se trouve pas en présence d'une essence autre. C'est notamment le cas pour l'A cajou, qui est l'essence la plus recherchée, sinon la plus connue, et qui existe à peu près partout dans toute l'ćtendue de la zone forestière. On en rencontre des peuplements assez denses tout près de la côte, dans la région à droite et à gauche de Bassam, depuis la frontière de la Gold Coast jusqu'à hauteur de Jacqueville; le cordon littoral lui-même en est assez fourni. Par contre, au fur et à mesure qu'on s'éloigne vers l'Ouest, il faut, pour trouver des $A$ cajous en nombre relativement important, remonter peu à peu au Nord. En face de Grand-Lahou, la zone à A cajou commence à 30 kilomètres environ de la côte; elle se maintient à cette distance puis redescend légèrement vers Fresco et vers Sassandra. Elle remonte progressivement ensuite pour aboutir à la frontière du Libéria à une quarantaine de kilomètres du littoral. Il est, d'autre part, à signaler qu'entre la zone à Acajou et la mer, depuis Grand Lahou jusqu'au fleuve Cavally, on trouve près du littoral plusieurs faux Acajous dont le Sipo, ayant l'aspect de l'Acajou mais ayant une contexture de bois un peu différente. Ce Sipo, qui est très rare dans la région de Bassam, est un bois qui peut très bien être vendu pour de l'Acajou; il est plus beau, à notre avis, que l'Acajou vrai qui est coupé près du littoral.

Au Nord, les limites de la zone à Acajou sont très irrégulières. Elles s'arrêtent en moyenne à 150 kilomètres de la côte mais remontent beaucoup plus haut dans les vallées, à 200 kilomètres et plus de la mer.

Les autres essences exploitables sont également disséminées un peu partout; elles n’ont

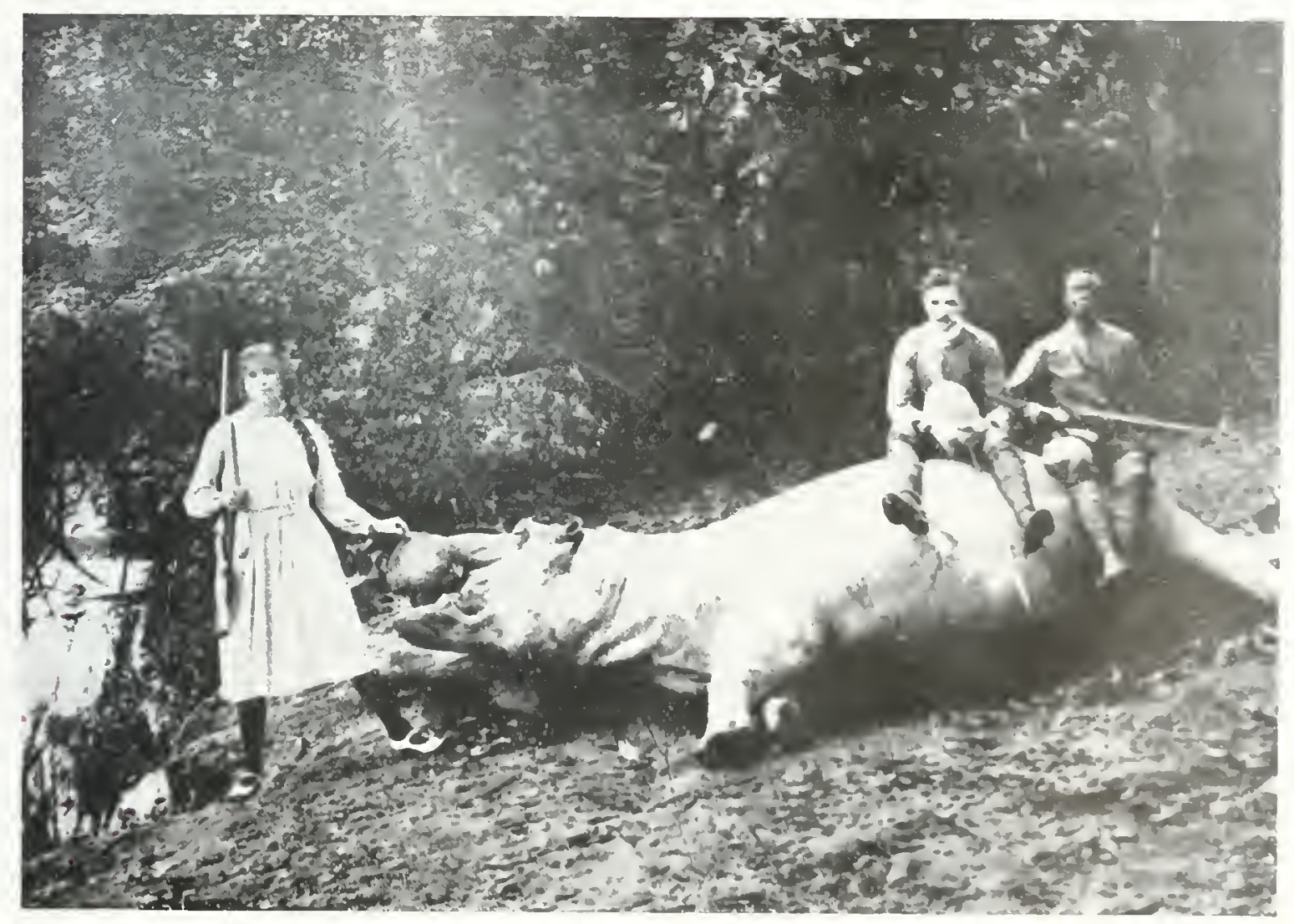

Chasse a l'hippopotame.

Cliché Burser 
pas d'habitat réservé. Faisons remarquer cependant que certaines d'entre elles, le Bahia, notamment, le Fou, le Rikio, se rencontrent plus particulièrement dans la zone côtière. Il

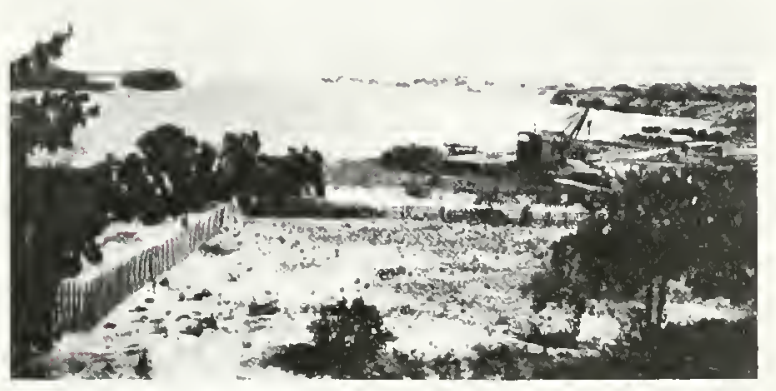

Vue de lentree du Comoe. - Plateau de Mossou Cliché Métayer - Grand-Bassam

existe de véritables forêts de Bahia près des lagunes, dans les terrains marécageux; c'est de beaucoup l'essence la plus facilement exploitable en très grosses quantités, bien que le sol spongieux ou inonde périodiquement, sur lequel elle pousse ne soit pas très favorable à son exploitation. Le Fou voisine fréquemment avec le Bahia mais on le rencontre aussi sans ce dernier, en peuplements assez denses, toujours dans le voisinage des rivièrez. Le Rikio est réparti dans toute la zone côtière; on dirait qu’il gagne peu à peu sur les savanes.

Deux autres essences, exceilentes également, et qui entrent pour une assez forte proportion dans la composition de la forêt, existent à peu près partout, dans la zone exploitable située un peu plus en arrière des lagunes, en peuplements plus ou moins denses: ce sont le Dabéma st l'Avodiré. Les renseignemenis que nous avons recueillis confirment, à très peu de choses près, sur ce point, les indications publiées par la mission Bertin.

Les autres essences sont généralement plus disséminées dans la forêt. Nous allons indiquer leur coefficient moyen de fréquence, d'après les reconnaissances effectuées par la mission précitée.

L'abaiage et l'utilisation de cette série de 39 essences auxquelles or pourrait encore ajouter, pour la pâte à papier, le Fromager et le Parasolier, permettraient d'exploiter plus de $60 \%$ du cube total pouvanì ètre fourni par la forêt. (Voir pages 23, 24 et 25.)

L'Inspecteur des Eaux et Forêts Bertin est le premier à reconnaitre, qu'en l'état actuel des choses, l'exploitation d'un aussi grand nombre d'essences diverses n'est pas possible. D'abord parce qu il serait extrêmement difficile d'imposer à l"industrie européenne une quarantaine de bois nouveaux, ensuice parce que beaucoup d'entre ces bois ont une valeur marchande trop faible pour pouvoir être écoulés avec bénéfice par les exploitants.

\section{Classement industriel}

Nombre de personnes, parmi ceiles qui ont eu à s'occuper plus ou moins des bois coloniaux, ont pensé à baptiser ces bois de noms

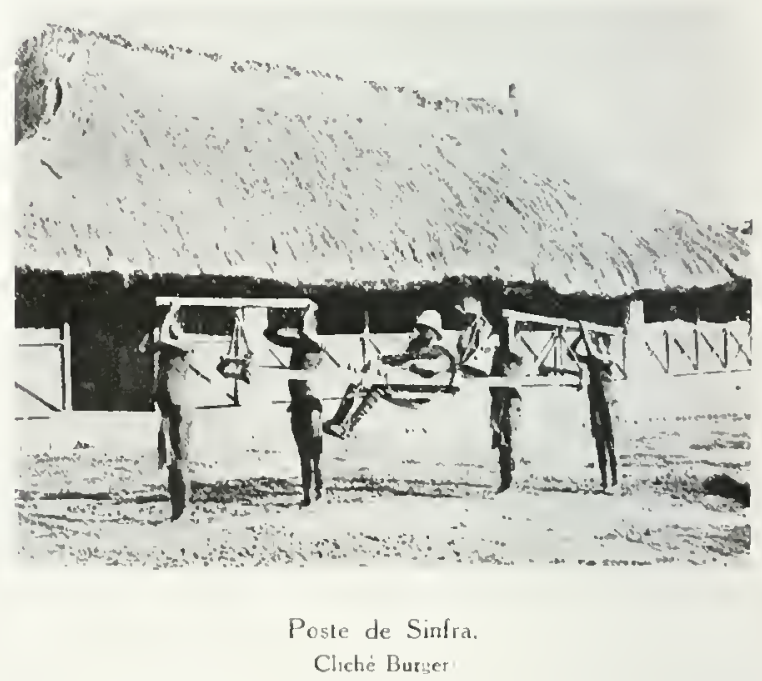

français, plus faciles à se rappeler que les noms un peu barbares sous lesquels ils sont connus. On a proposé : chêne blanc, blond ou brun d'Afrique; sapin d'Afrique, hềire rouge ou gris 


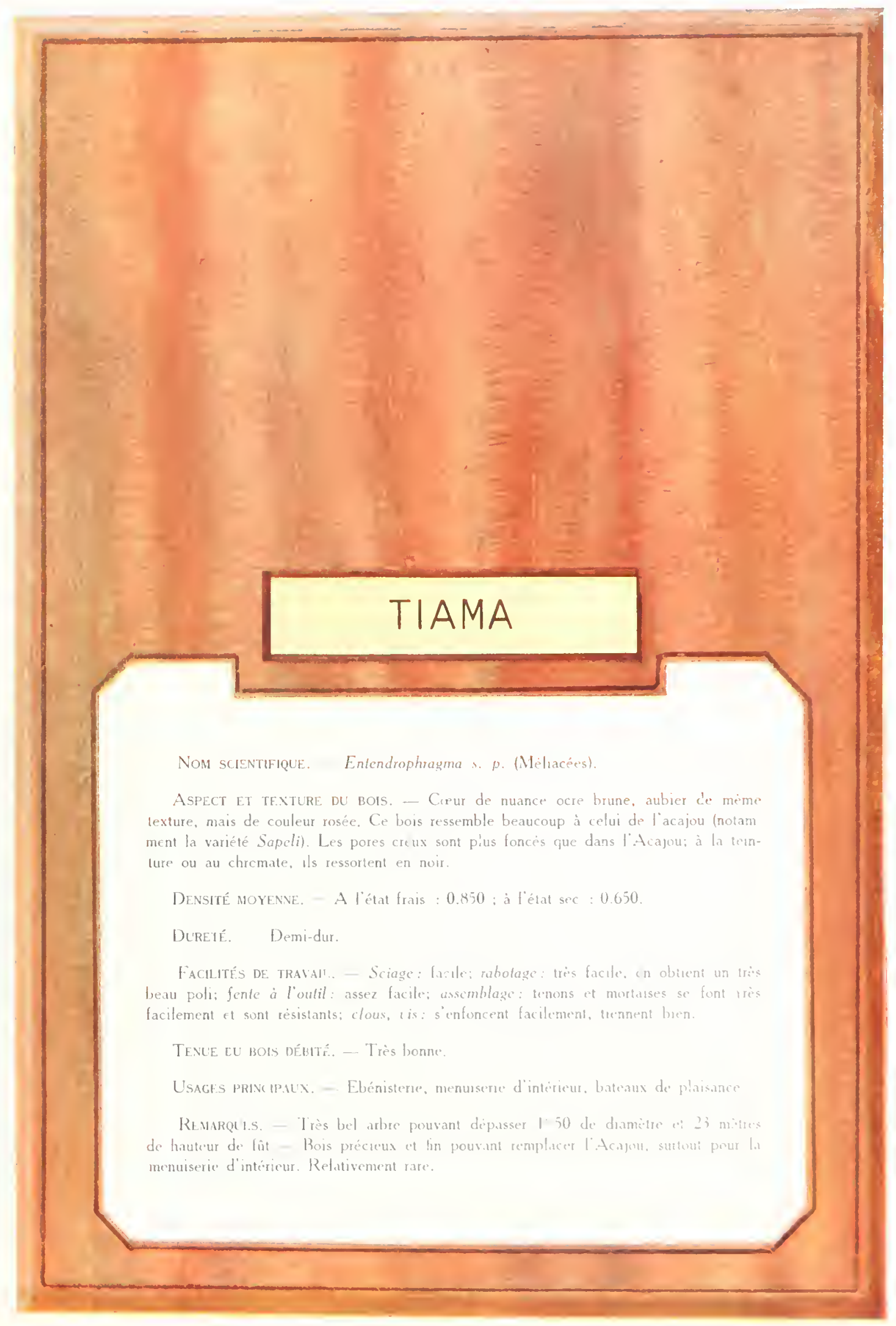


. 
COEFFICIENTS DE FREQUENCE OBTENUS DANS LES PROSPECTIONS DE LA MISSION BERTIN I

\begin{tabular}{|c|c|c|c|c|c|}
\hline $\begin{array}{l}\text { Numéro } \\
\text { d'ordre } \\
\text { el lellres } \\
\text { de serve } \\
1 \\
\end{array}$ & $\begin{array}{l}\text { NO.1 USUEL } \\
\text { adopté } \\
2 \\
\end{array}$ & $\begin{array}{c}\text { NOM SCIENTIFIQLE } \\
\text { Famille } \\
3\end{array}$ & $\begin{array}{r}\text { NEMS VERNACLLAIRES } \\
4 \\
\end{array}$ & 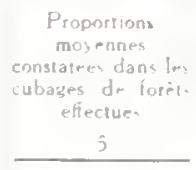 & $\begin{array}{l}\text { OBSERVA TIONS } \\
{ }_{6}^{6}-\end{array}$ \\
\hline $\begin{array}{l}1 \\
\text { A }\end{array}$ & $\begin{array}{c}\text { ACAJOU } \\
\text { dit de } \\
\text { GRAND.BASSAM }\end{array}$ & $\begin{array}{l}\text { Khaya iurensis } \\
\text { (Méliacees) } \\
\text { Chevalier, p. } 207\end{array}$ & $\begin{array}{l}\text { Ehbié ou Ekhoie ou Ecguéthie (abé) } \\
\text { Humpé (ebre). Duuhouma (agni). Dubrri } \\
\text { ou Kéguiga (apoll.). Biribu (bariba) } \\
\text { Dukuma Dugura (agnal, Lokpobua (attie). }\end{array}$ & 1. - & \\
\hline $\begin{array}{l}2 \\
\mathrm{~B}\end{array}$ & $\begin{array}{l}\text { TIANA ou } \\
\text { ACAJOU.TIAMA } \\
\text { (abe) }\end{array}$ & $\begin{array}{l}\text { Entandrophragma } \\
\text { sp. (Meliacees) } \\
\text { Chevalier, } 195 \text { a } 207 .\end{array}$ & $\begin{array}{l}\text { Tiama-liama lapoll. el agni). Onabnu ou } \\
\text { Lokaha ou Lokobo (atte). Baka-Biringui } \\
\text { (abe) (c.-a-d. le roi de la foret). }\end{array}$ & & $\begin{array}{l}\text { Priporion } \\
\text { non determinet. } \\
\therefore \text { ensiron. }\end{array}$ \\
\hline $\begin{array}{l}3 \\
\mathrm{C}\end{array}$ & $\begin{array}{l}\text { ÄÉE.E. } \\
\text { (apoll.) }\end{array}$ & $\begin{array}{c}\text { Canarium accidentale } \\
\text { (Burceracees) } \\
\text { Chevalier, p. } 145 \text {. }\end{array}$ & $\begin{array}{l}\text { Labé (abe). Sénian ou Segna (attie) } \\
\text { Krandju-haügué (agni). Yalu (plapo). }\end{array}$ & 1.5 & \\
\hline D & $\begin{array}{c}\text { AkJ } \\
\text { (bonoua) }\end{array}$ & $\begin{array}{l}\text { Antiaris tonicaria } \\
\text { (Lincacees) } \\
\text { Chevaluer. p. } 259 \text {. }\end{array}$ & $\begin{array}{l}\text { 1hédé (abel Mbonpou (atie). Wulie } \\
\text { (abe)? }\end{array}$ & & $\begin{array}{l}\text { Proportion } \\
\text { non delerminee. } \\
\therefore \quad \text { a } 1\end{array}$ \\
\hline $\begin{array}{l}5 \\
E\end{array}$ & $\begin{array}{l}\text { ANIONKETI } \\
\text { (abe) }\end{array}$ & $\begin{array}{c}\text { Puchypodanthium } \\
\text { Staudtii } \\
\text { (Annnacees) } \\
\text { Chevalier, p. } 118\end{array}$ & Miedzo (abe) & & $\begin{array}{l}\text { Praportunn } \\
\text { non determinee. } \\
\vdots \text { a } 1\end{array}$ \\
\hline F & $\begin{array}{c}\text { ASAS } \\
\text { (pahouin) }\end{array}$ & $\begin{array}{l}\text { Bridelia speciosu } \\
\text { (Euphorbiacees) } \\
\text { Chevalier. p. } 158 .\end{array}$ & $\begin{array}{l}\text { Chiukaué (abe). Thlikacé ou (hikue } \\
\text { (altie). Epatontroubu (a-m). }\end{array}$ & $\begin{array}{l}\text { Peuriements } \\
\text { lies laubles }\end{array}$ & \\
\hline $\begin{array}{l}7 \\
\mathrm{G}\end{array}$ & $\begin{array}{l}\text { AVODIRE } \\
\text { (apoll.) }\end{array}$ & $\begin{array}{c}\text { Bingeria africana: } \\
\text { (Meliacees) } \\
\text { Chevalier, p. } 189 .\end{array}$ & $\begin{array}{l}\text { Aghoui (abe). Hagué (agni). Hakue } \\
\text { (allé). }\end{array}$ & 2.5 & \\
\hline $\begin{array}{l}8 \\
\mathrm{H}\end{array}$ & $\begin{array}{l}\text { BADI } \\
\text { (attie) }\end{array}$ & $\begin{array}{c}\text { Sarcocephalus Poheguini } \\
\text { (Rubiacees) } \\
\text { Chevaluer. p. 231. }\end{array}$ & $\begin{array}{l}\text { Bédo (abél. N'Débéré (attue). Ekusumba } \\
\text { lappoll.l. Boissima ou Boisima (agni). } \\
\text { Zérongo (bambara). }\end{array}$ & $\begin{array}{l}\text { Peuplements } \\
\text { lies latbles. }\end{array}$ & \\
\hline 9 & $\begin{array}{l}\text { BAHIA } \\
\text { (apoll.) }\end{array}$ & $\begin{array}{l}\text { Mitrugyne mocrophyllo } \\
\text { (Rubiaces) } \\
\text { Chevalier. p. } 228 .\end{array}$ & $\begin{array}{l}\text { Soufo (abe). Ciofo lebric). Alchuipan } \\
\text { (bonova). Bosn (brignan). Sofo (attie). }\end{array}$ & 10 & 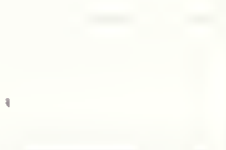 \\
\hline $\begin{array}{l}10 \\
J\end{array}$ & $\begin{array}{l}\text { BOSSÉ } \\
\text { (abe) }\end{array}$ & $\begin{array}{l}\text { Trichilia cedrota } \\
\text { (Nielracees) } \\
\text { Chevalicr. p. } 214\end{array}$ & $\begin{array}{l}\text { Krassé ou Cuanuhé (abe). MBossa } \\
\text { (apoll.). Krassé ou 'Dzand latlie). MBossé } \\
\text { (apoll. agni). Anokué (tornoud). }\end{array}$ & 0,2 & $\checkmark$ \\
\hline $\begin{array}{l}11 \\
K\end{array}$ & $\begin{array}{l}\text { DABE.IA } \\
\text { (apoll.) }\end{array}$ & $\begin{array}{c}\text { Piptadenia africana? } \\
\text { (Legumineuses) } \\
\text { Chevalier, p. 182. }\end{array}$ & $\begin{array}{l}\text { Ehć (abe). ('Bon (aulie). Ahé (ébrue). } \\
\text { Kuanga-iniama (agni). Noinvi (bon- } \\
\text { doukou). }\end{array}$ & 7. & \\
\hline $\begin{array}{l}12 \\
\mathrm{~L}\end{array}$ & $\begin{array}{l}\text { FARO } \\
\text { (abe) }\end{array}$ & Indetermune. & & & $\begin{array}{l}\text { Proportion non } \\
\text { delerminee }\end{array}$ \\
\hline
\end{tabular}

(1) Ce sont ces caeffients que nous adopterons pour etuduer les posstblites d'ecplontation des bors enumeres. Wais ils ne

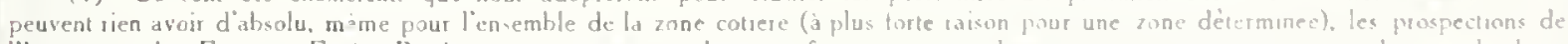

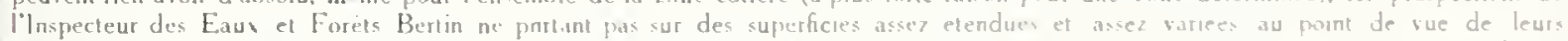

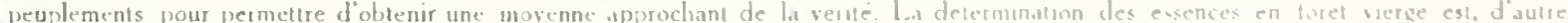

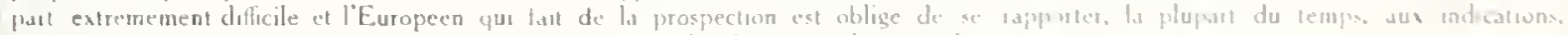
quelquetois vogues ou lantoisistes, que lui louma le personnel indigene conploye par lut 


\begin{tabular}{|c|c|c|c|c|c|}
\hline $\begin{array}{l}\text { V.mero } \\
\text { dordie } \\
\text { et listress } \\
\text { de série } \\
\text { I }\end{array}$ & $\begin{array}{l}\text { NOM USUEI. } \\
\text { adopie } \\
2 \\
\end{array}$ & $\begin{array}{c}\text { NOM S IENTIFIQUE } \\
\text { Famil } \\
3 \\
\end{array}$ & $\begin{array}{c}\text { NOMS VERNACULAIRES } \\
4\end{array}$ & $\begin{array}{c}\text { P'ropotions } \\
\text { moyennes } \\
\text { conatatees dans les } \\
\text { cubines de torets } \\
\text { efecturs } \\
5 \\
\end{array}$ & $\begin{array}{c}\text { OBSERVATIONS } \\
6 \\
\end{array}$ \\
\hline $\begin{array}{l}13 \\
\mathrm{MJ}\end{array}$ & $\begin{array}{r}\text { FRAKEE } \\
\text { (agni) }\end{array}$ & $\begin{array}{l}\text { Terminalia allissima } \\
\text { (Combrelacees) } \\
\text { Chevalier. p. } 151 .\end{array}$ & Pé abe). Té(atlie). Fram (bondusukou). & $\begin{array}{l}\text { Peuplements } \\
\text { tres lables }\end{array}$ & \\
\hline $\begin{array}{l}14 \\
N\end{array}$ & $\begin{array}{l}\text { FRAMIRE } \\
(\text { apoll., agni) }\end{array}$ & $\begin{array}{l}\text { Terminulia ivorensis } \\
\text { (Combrelacees) } \\
\text { Chevalier, p. } 152 .\end{array}$ & $\begin{array}{l}\text { Boli ou MBoli (abe) Bona " " Buna } \\
\text { ou Yapi (allie). Caüri (bonona) Amhidju } \\
\text { (bondoukou). }\end{array}$ & 0.6 & \\
\hline $\begin{array}{l}15 \\
0\end{array}$ & $\begin{array}{l}\text { IROKO } \\
\text { (ironous) }\end{array}$ & $\begin{array}{l}\text { Chlorofora ercelsa } \\
\text { (Unlicacees) } \\
\text { Chevaliet, p. } 261 .\end{array}$ & 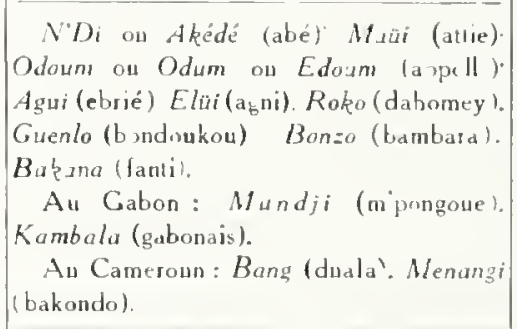 & 3.- & \\
\hline $\begin{array}{l}16 \\
P\end{array}$ & $\begin{array}{l}1.0 \\
\text { (abe el allie) }\end{array}$ & $\begin{array}{l}\text { Purkia agboensis? } \\
\text { (1.esumineuses) } \\
\text { Chevalier, p. 181. }\end{array}$ & $\begin{array}{l}\text { Assamä (appoll bonoua). Dezo (bon- } \\
\text { doukou) }\end{array}$ & 2.5 & \\
\hline $\begin{array}{l}17 \\
(2\end{array}$ & $\begin{array}{c}\text { MAKORE } \\
\text { (aioil.) }\end{array}$ & $\begin{array}{l}\text { Dimariu Heckeli } \\
\text { (S. wotacees) } \\
\text { Chevalier, P. 237. }\end{array}$ & 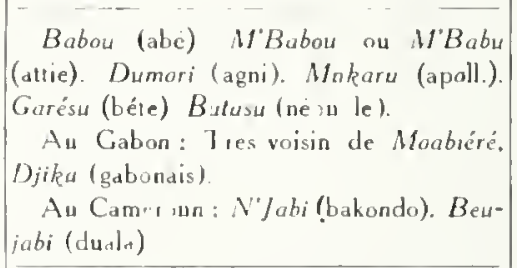 & 0.4 & \\
\hline $\begin{array}{l}18 \\
\mathrm{R}\end{array}$ & $\begin{array}{l}\text { NI ANGON } \\
\text { (dipoll.) }\end{array}$ & $\begin{array}{l}\text { Cala proleiformis } \\
\text { (Sierculiaces:) } \\
\text { Chevalieq, p. } 250 .\end{array}$ & $\begin{array}{l}\text { Banda (abe). Kuanda ou Kouandu } \\
\text { (alle) Kekosi (ebrie). Kohotst (tanti) } \\
\text { Viangan ou Gnionson (agni). }\end{array}$ & 1. & \\
\hline $\begin{array}{c}19 \\
S\end{array}$ & $\begin{array}{l}\text { R K1O } \\
\text { (atie) }\end{array}$ & $\begin{array}{c}\text { Uupuca bingervillensis } \\
\text { (En lionbiacees) } \\
\text { Chevalue, p. 162. }\end{array}$ & $\begin{array}{l}\text { Var ou Na ou Giembi (allie). Alukaha } \\
\text { (appoll). Alébié (ebrie) Klékhua (agnu). } \\
\text { Enébien (brigran) Aluba oll Alabo cu } \\
\text { Orato (bonnua). Kaya (bondoukou). }\end{array}$ & 5. - & \\
\hline $\begin{array}{l}\text { 2) } \\
\mathrm{T}\end{array}$ & $\begin{array}{l}\text { SENAN } \\
\text { (Alle) }\end{array}$ & $\begin{array}{c}\text { Muecaba'rya slupfia-a? } \\
\text { (Euphorbiaces) } \\
\text { Chesdier, p. } 162 \text {. }\end{array}$ & $\begin{array}{l}\text { Djilika au Djirite (abe). Emuin quin } \\
\text { ou Tuanga (apoli.). Assa-bugué (agnı) } \\
\text { Sonia (bonnud) Bapi (brignan) }\end{array}$ & 0.8 & \\
\hline $\begin{array}{l}21 \\
U\end{array}$ & 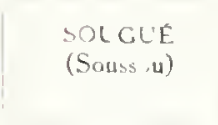 & $\begin{array}{l}\text { Purincrium tenuifolium } \\
\text { (Rosacees) } \\
\text { Chevalies. P. } 225 \text {. }\end{array}$ & $\begin{array}{c}\text { So (abe) Moussé on Simur (allie) } \\
\text { Faoulé-k.kole (a.joll.). Catésima (bonoua). }\end{array}$ & 3.- & \\
\hline$\frac{22}{V}$ & $\begin{array}{c}\text { TALi } \\
\text { (malinhe) }\end{array}$ & $\begin{array}{c}\text { Eruthrophlesum guineense } \\
\text { (Legumineuses) } \\
\text { Chevalies, p. } 179 .\end{array}$ & $\begin{array}{l}\text { Guié ou NGGuié (abe). Lo (atie) } \\
\text { Erhoné ou Arhoné (apoll.) Aliẻma (ebrie) } \\
\text { Erüi (agnı) Elégué-mouani (bon ua). Téli } \\
\text { (bambura) }\end{array}$ & 2.5 & \\
\hline $\begin{array}{c}31 \\
A A\end{array}$ & $\begin{array}{l}A B+1 E \\
(a, n i)\end{array}$ & $\begin{array}{l}\text { Petersia viridifora } \\
\qquad \text { (Myitacees) } \\
\text { Chevalier, p. } 301 .\end{array}$ & $\begin{array}{l}\text { Kuli nu Kati (abe) Kan (allie). Assiga } \\
\text { (apoll.). Abimpé (ebrie). Essivé ou Abalé } \\
\text { (agni). Esivé (bonoua). }\end{array}$ & 2. - & \\
\hline $\begin{array}{l}32 \\
1313\end{array}$ & $\begin{array}{l}\text { ADJA } \\
\text { (honous) }\end{array}$ & $\begin{array}{l}\text { Cicca discaïdeus } \\
\text { (Euphorbiacees) } \\
\text { Cheralier, p. } 273 .\end{array}$ & $\begin{array}{l}\text { Moussan-koué ou Mausan-koé, Mansan- } \\
\text { haué ou Mu>a-noué (abe, allie). Ténoubar } \\
\text {-u Pépésiu (apoll) Adjansé (bonuu.ı) } \\
\text { Brakassa (ebrie). }\end{array}$ & 1.5 & \\
\hline
\end{tabular}




\begin{tabular}{|c|c|c|c|c|c|}
\hline $\begin{array}{l}\text { Numbio } \\
\text { dordir } \\
\text { ot lettiea } \\
\text { de télie } \\
1 \\
\end{array}$ & $\begin{array}{l}\text { NOM LiSLE.I } \\
\text { adopir } \\
2 \\
\end{array}$ & $\begin{array}{l}\text { NOM SCHNTIFIOLE } \\
\text { l'ammle } \\
3 \\
\end{array}$ & $\begin{array}{c}\text { NOMS VIRNACUL IIRES } \\
4\end{array}$ & 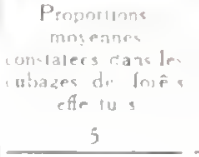 & $\begin{array}{l}\text { ()B-ERTATION } \\
6 \\
\end{array}$ \\
\hline $\mathrm{C}^{33} \mathrm{C}$ & $\begin{array}{l}\text { ADJOU A BA } \\
\text { (a)suli.) }\end{array}$ & $\begin{array}{c}\text { Hacmoloshuphis Banteri } \\
\text { (Anacirdacees) } \\
\text { Chevaluer, p. } 113 \text {. }\end{array}$ & 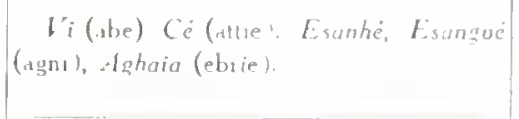 & 2.5 & \\
\hline $\begin{array}{l}3+ \\
D D\end{array}$ & $\begin{array}{l}\text { ANINGUERI } \\
\text { (abe) }\end{array}$ & $\begin{array}{l}\text { Molancalios robusta } \\
\text { (Sapolacees) } \\
\text { Chevaliee. P. } 241\end{array}$ & $\begin{array}{l}\text { Alokotimon ou Aloknslumo:i (aplie) } \\
\text { Aosumé ou Auamé (ans) }\end{array}$ & & 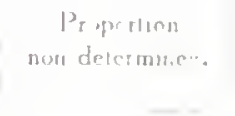 \\
\hline $\begin{array}{l}35 \\
\mathrm{E} E\end{array}$ & $\begin{array}{l}\text { APOME } \\
\text { (allie) }\end{array}$ & Indetermise. & $\begin{aligned} \text { Bata-kounini (dimoll) } \\
\end{aligned}$ & 1.5 & \\
\hline $\begin{array}{l}36 \\
F F\end{array}$ & $\begin{array}{l}\text { (ZOBO } \\
\text { (apoll.) }\end{array}$ & $\begin{array}{l}\text { Lophira procera } \\
\text { (Dipterucarpess) } \\
\text { Chevalter, p. } 154 .\end{array}$ & 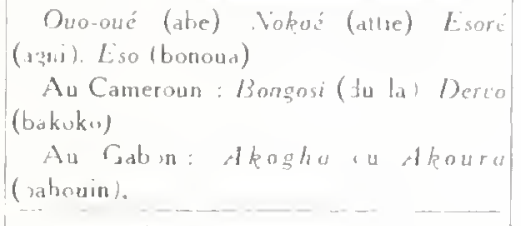 & 4. - & \\
\hline $\begin{array}{l}37 \\
\mathrm{C} G\end{array}$ & $\begin{array}{l}\text { BODIOA } \\
\text { (apull.) }\end{array}$ & $\begin{array}{l}\text { Pynacrlid accidentalis } \\
\text { (Veliacees) } \\
\text { Chevaler. 0. } 211 .\end{array}$ & 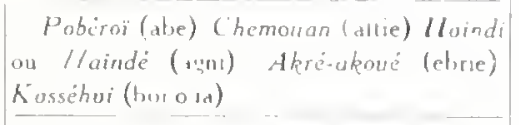 & 1.5 & \\
\hline 1111 & $\begin{array}{l}\text { COLI.A } \\
\text { (gabon) }\end{array}$ & $\begin{array}{l}\text { Coulu edulis } \\
\text { (Olaciness) } \\
\text { (hevalier, p. } 218 .\end{array}$ & 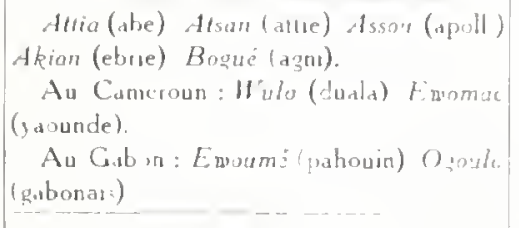 & 1. - & \\
\hline $\begin{array}{l}39 \\
11\end{array}$ & $\begin{array}{c}\text { EVIILN } \\
\text { (apoll., agni) }\end{array}$ & $\begin{array}{l}\text { Alstonia congensis } \\
\text { (Ajocynees) } \\
\text { 'hevalier, n. } 121\end{array}$ & 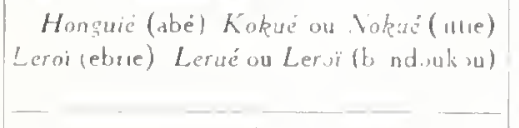 & $\begin{array}{l}\text { Peuplemenis } \\
\text { Ires fables }\end{array}$ & \\
\hline $\begin{array}{r}40 \\
-\end{array}$ & $\begin{array}{l}\mathrm{FOU} \\
\text { (abe) }\end{array}$ & $\begin{array}{l}\text { Ol fieldia africunos } \\
\text { (F uphosbiaces) } \\
\text { Chevalier, r. } 160 \text {. }\end{array}$ & $\begin{array}{l}\text { Essonou Fu (atlie) Angosuran (ebrie) } \\
\text { Esui (agni) Esron-angouarian ou Eiur } \\
\text { (bonoua) }\end{array}$ & 1.5 & \\
\hline $\begin{array}{c}41 \\
K K\end{array}$ & $\begin{array}{l}\text { KROMA } \\
(a m i l)\end{array}$ & $\begin{array}{l}\text { Klainedora } \\
\text { (Irvingiacees) }\end{array}$ & Aquabo'abe, atlie) Adroumtenu: (ebue) & $1, ;$ & \\
\hline $\begin{array}{r}42 \\
1 \mathrm{~L}\end{array}$ & $\begin{array}{l}\text { OBOTO } \\
(y \text { ibon) }\end{array}$ & $\begin{array}{l}\text { Ochrosarpus africanus } \\
\text { "H Mommez africana } \\
\text { (Gultif ies) } \\
\text { Chevalier p. } 165 .\end{array}$ & 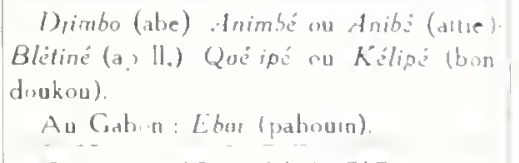 & 0.4 & \\
\hline$\stackrel{43}{\mathrm{M} \mathrm{M}}$ & $\begin{array}{l}\text { OLON } \\
\text { (pab uin) }\end{array}$ & $\begin{array}{l}\text { Fagara macrophylla } \\
\text { (Rulacees) } \\
\text { Chevalier. p. } 232 \text {. }\end{array}$ & 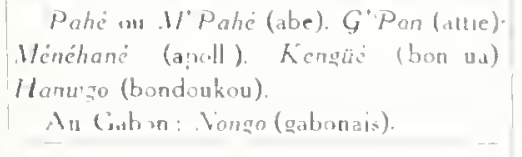 & & 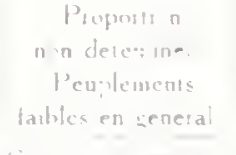 \\
\hline 44 & PALETUVIER & 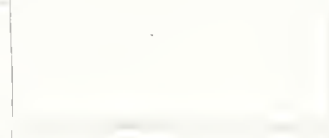 & & 1,5 & 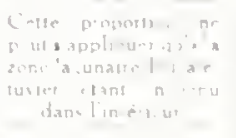 \\
\hline 45 & $\begin{array}{c}\text { PRI } \\
\text { (outue de la } \\
\text { lagune potou) }\end{array}$ & $\begin{array}{l}\text { Funtumia africano } \\
\text { (tpocynets) } \\
\text { Clievilier, p } 122 \text {. }\end{array}$ & 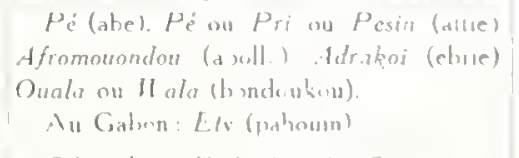 & & $\begin{array}{l}\text { Promostum } \\
\text { num ielkmanace }\end{array}$ \\
\hline 46 & $\begin{array}{c}\text { SAMBA } \\
\text { (bsuroukiou) }\end{array}$ & $\begin{array}{c}\text { Triplozhito sclerovilon } \\
\text { (Sterculiaces) } \\
\text { Chevilier, p 255. }\end{array}$ & 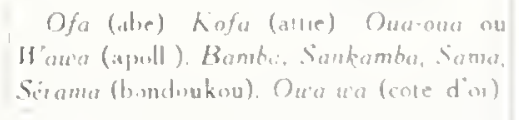 & I. & \\
\hline 47 & $\begin{array}{l}S B O \\
(i ; n o l l .)\end{array}$ & 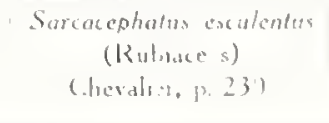 & 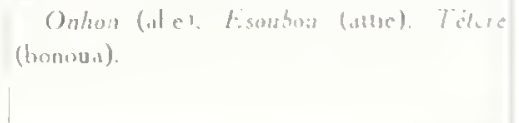 & & \\
\hline
\end{tabular}


d'Afrique (1), etc... Ces appellations, nous dit M. Bertin (2) " eussent certes été commodes; mais dans la pratique elles sont impossibles, la gamme de nos bois d'Europe n'érant pas assez variée pour permettre des similitudes complètes entre les deux collections. Lorsque les couleurs se rapprochent, la densité diffère, et réciproquement; de même quand la dureté est semblable, les résistanceś à la flexion ne concordent plus ".

Nous dirons mieux, d'accord en cela avec M. Gillet, l'industriel bien consu, qui depuis plus de dix ans, expérimente et utilise les bois de la Côte d'lvoire et du Gabon. Il seraii dangereux de qualifier "chêne " - ce nom fûtil suivi d'un adjectif et du pays de provenance - un bois qui scientifiquement n'est pas du chêne. La seule crainte de se voir engagés dans des procès si ces bois ne donnaient pas complète satisfaction, ferait hésiter le $\mathrm{e}_{3}$ architectes à les employer. Et puis ce serait la porie ouvert à toutes les fraudes: le chêne d'Afrique n'étant pas déterminé scientifiquement, certains marchands peu consciencieux ou insuffisamment éclairés, livreraient pour du chêne d'Afrique des bois de qualité inférieure, provenant on ne sait d'où. L'idée est donc à rejeter. Par contre, il est naturel qu'on fasse des rapprochements entre les essences africaines et celles d'Europe, ne serait-ce que pour indiquer l'emploi dont les premières sont susceptibles.

A ce sujet, les représentants des Chambres syndicales du Bàtiment, de la Menuiserie, de la Carrosserie, du Charronage ei de l'Ameublement, réunis en "Commission officielle des Bois Coloniaux ", ont adopté, pour les bois de la Côte d'lvoire, une classification en six catégories des essences retenues par la Mission Bertin (3) :

7 essences de densité variant de 0.270 à

(1) Nous-mêmes n’avons pas échappé à cette erreur. (Rapport au Directeur de l'Agence Economique de l'A. O. F.. sur la foire d'échantillons de Lyon.)

(2) Notice : Les Bois Coloniaux.

(3) Bertin : notice déjà citée.
0.625, couleurs allant du blanc uni au blanc grisâtre, pouvant remplacer le peuplier, le grisard et le tulipier d'Amérique;

6 essences de densité comprise entre 0.450 et 600 , couleurs blanc rosé ou jaunâtre pouvant remplacer les pins et sapins;

8 essences, densité 0.610 à 875 , couleurs rose, rouge, grise, jaune ou brune, pouvant remplacer le chêne et le teck;

2 essences, densité 0.675 et 0.750 , couleurs rougeâtre ou gris-violacé, analogues au hêire et au platane;

7 essences, densité 0.575 à 0.950 , aux belles couleurs variées, allant du jaune d'or au brun-rouge foncé, essences de toute première qualité pour l'ébénisterie et le placage:

Enfin 9 essencez, dont l'usage n'a pas été bien déterminé, mais qui, pai leur dureté, leur résistance à la flexion, à l'arrachement, peuvent être utilisées pour des traverses de chemins de fer, matéricl roulant, pilotis, constructions navales, eic.

Nous résumons cette classification dans !e tableau ciraprès :

Classement commercial des princizaux bois de la Côte d'luoire, adopté par la Commission offi cielle des Bois coloniaux (1).

PREMIERE CATEGORIE

Bois tendres pouvant remplacer le peuplier, le grisard, le tulipier d'Amérique.

Emplois: Ménuiserie légère. caissene, contreplacage et travaux d'interieur n'exigeant pas beaucoup de resistance.

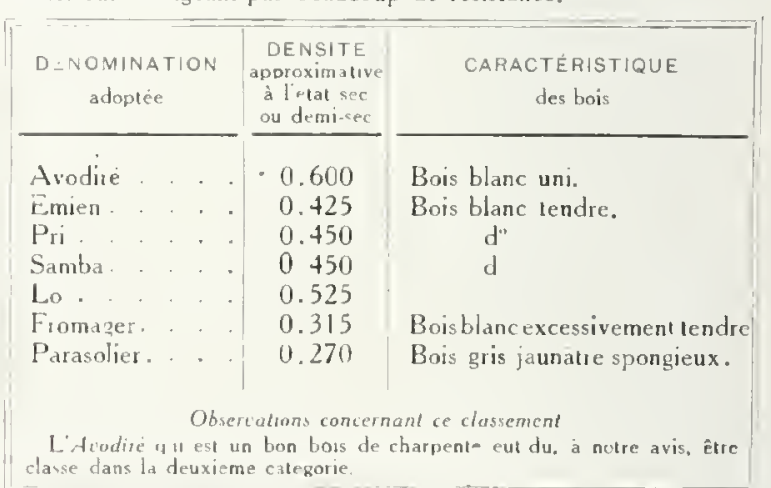

(1) La Commission d'essais des Bois coloniaux, qui fonctionnait au Minis'ère des Colonies, en 1918, avait même classé cette essence parmi les bois de tournerie et d'ébénisterie. (Séance du 26 octobre 1916.) 


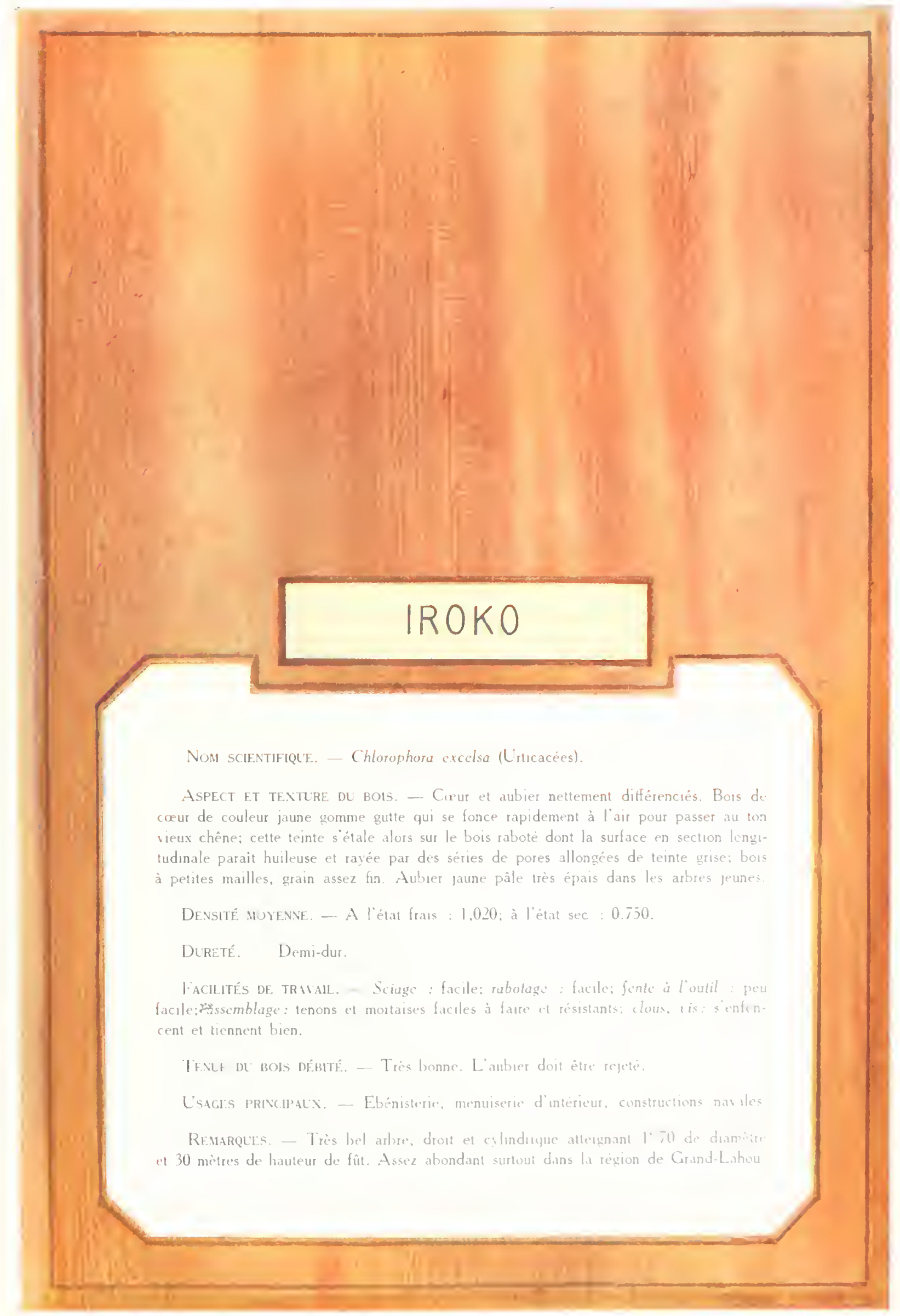


I 
DEUXIENE CATECORIE

Bois pouvant remplacer les bois et sapun:

Emplois: Menuiseric, charpente.

\begin{tabular}{|c|c|c|}
\hline $\begin{array}{l}\text { DENOMINATION } \\
\text { sdoplee }\end{array}$ & $\begin{array}{c}\text { OENSITE } \\
\text { approximative } \\
\text { à lotat sec } \\
\text { ou dem }\end{array}$ & $\begin{array}{l}\text { CARACTERISTIQUE } \\
\text { des boi }\end{array}$ \\
\hline Aielé & 0450 & $\begin{array}{l}\text { Bu, tose nes pale, grain de } \\
\text { lincajus. }\end{array}$ \\
\hline Bahia & 0550 & $\begin{array}{l}\text { Bors gris orange fonce et } \\
\text { uni. }\end{array}$ \\
\hline Framire & 0475 & Bols jaune dais. \\
\hline Niangon & 0.575 & Bols rose saumone. \\
\hline Sibo. & $0 \$ 75$ & Bois faune orange. \\
\hline
\end{tabular}

TROISIEME CATÉGORIE

Bois pouvani remplacer les diverses vaijetes de chene el de teck Emplois: Constructuon, menuiserie de batıment, parquets, char pentes, pilotis, poutres, materiel de chemin de fer, constructions navales.

\begin{tabular}{|c|c|c|}
\hline $\begin{array}{l}\text { DENOMINATION } \\
\text { adoptée }\end{array}$ & $\begin{array}{l}\text { DENSITE } \\
\text { appioximalise } \\
\text { a l'elat se: } \\
\text { ou demi-ec }\end{array}$ & $\begin{array}{c}\text { CARACTERISTIQUE } \\
\text { des boIs }\end{array}$ \\
\hline $\begin{array}{l}\text { Dabema . . } \\
\text { Iroko } \\
\text { Makore } \\
\text { Niangon } \\
\text { Olon } \\
\text { Sougue. } \\
\text { Tali. } \\
\text { Fraké }\end{array}$ & $\begin{array}{ll}0 & 775 \\
0 & 70 ! 1 \\
0 & 725 \\
0 & 575 \\
0 & 875 \\
0 & 850 \\
0 & 875 \\
0 & 610\end{array}$ & $\begin{array}{l}\text { Bois gris jaunatre. } \\
\text { Bois jaune brun clair. } \\
\text { Rois rouge rose. } \\
\text { Bois rose saumone. } \\
\text { Bois jaune citron. } \\
\text { Bois couleur liege. } \\
\text { Bois brun clair. } \\
\text { Bois blanc jaunatie. }\end{array}$ \\
\hline \multicolumn{3}{|c|}{ 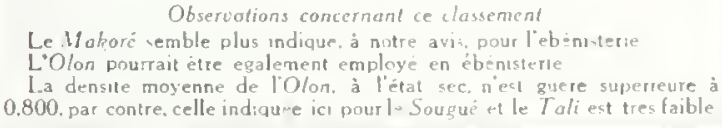 } \\
\hline
\end{tabular}

QUATRIEME CATÉGORIE

Bois pouvant remplacer le hetre et le platane.

Emplois: Tournerves bois a brosses, a paves, hourrellene, sieges.

\begin{tabular}{|c|c|c|}
\hline $\begin{array}{l}\text { DENUMINATION } \\
\text { adoptée }\end{array}$ & $\begin{array}{l}\text { DENSITE } \\
\text { approximative } \\
\text { à l'état sec } \\
\text { ou demi-sec }\end{array}$ & $\begin{array}{l}\text { CARACTERISTIQUE } \\
\text { des bois }\end{array}$ \\
\hline $\begin{array}{l}\text { Sénan. } \\
\text { Rikio. }\end{array}$ & $\begin{array}{ll}0 & 675 \\
0 & 750\end{array}$ & $\begin{array}{l}\text { Bois gris violace. } \\
\text { Bois brun rougeatre. }\end{array}$ \\
\hline
\end{tabular}

CINQUIEME CATEGORIE

Bots d'ebenisterie et de placage.

Emplois: Menuisere de luxe, ameublements.

\begin{tabular}{|c|c|c|}
\hline $\begin{array}{l}\text { DENOMINATION } \\
\text { adoplee }\end{array}$ & $\begin{array}{c}\text { DENSITE } \\
\text { approximalive } \\
\text { a !'etal sec } \\
\text { ou demi sec }\end{array}$ & $\begin{array}{c}\text { CARACTERISTIQUE } \\
\text { des boIs }\end{array}$ \\
\hline
\end{tabular}

Acajou .

Acajou Tiama

Apomé.

0625 Birs louge saumone.

Assas

0750 Bois rouge biun.

0 950 . Bots brun tose.

Badi

Bossé

0.575 Bois couleur tabese.

0.775 Bois faune d'or.

0.625 Bois rove pale.

Obolo. 0775 Ros touge rose fonce.

Obsertatrons concernant ie classement

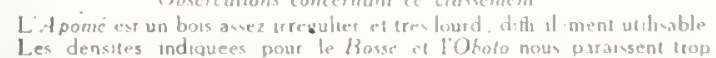
farbles

\section{- XIL VE CATÉGORIE}

Bois pouvant ente uthlises pour traverses de chen in de for, pilotis, etc.

\begin{tabular}{|c|c|c|}
\hline $\begin{array}{c}\text { DEMOMINATION } \\
\text { adoplé }\end{array}$ & $\begin{array}{c}\text { DENS TE } \\
\text { appoximative } \\
\text { à letar we } \\
\text { ou deminec }\end{array}$ & $\begin{array}{c}\text { CARACTER STIQUE } \\
\text { der bois }\end{array}$ \\
\hline $\begin{array}{l}\text { Abale } \\
\text { Adjansi } \\
\text { Adjouaba. } \\
\text { Adzobe } \\
\text { Bodiod. } \\
\text { Coula } \\
\text { Fou. } \\
\text { Kioma. } \\
\text { Paletuver }\end{array}$ & $\begin{array}{ll}0 & 750 \\
0 & 850 \\
1 & 050 \\
1 & 075 \\
11 & 9501 \\
1 & 075 \\
1 & 075 \\
1 & 050 \\
1 & 125\end{array}$ & $\begin{array}{l}\text { Bois rose. } \\
\text { Bois pune grsatre. } \\
\text { Bois gris brun faunatre. } \\
\text { Bois brun volace. } \\
\text { Bors paune ocre. } \\
\text { Bors brun lie de vin. } \\
\text { Bois rouge volace. } \\
\text { Bors brun jaunatse. } \\
\text { Bois rouge violacé. }\end{array}$ \\
\hline
\end{tabular}

$$
\text { Obierrations rancrernant ce clastement }
$$

L. Adrougabo pourra peuteetre éry classé par la sutte dans lea bass de

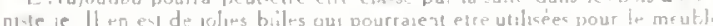
moderne

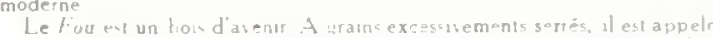
a rempla er le- bus, cormer et roalak. pour l'outiliage el divers rmplo:s speciaus

Nota. - Le classement de lAko, de l'Anoukéll et de l Antguery semble ouoir été omis por la Commission.

Les deux premières exsences doicent, à notre ocis, étre ujoutées à la deurième catégonce. La troisième à la troisième colégorie.

Nous verrons plus loin. dans la troisième partie de ce travail, que. sauf en ce qui concerne l'Avodiré qui est un bois d'excellente qualité. les essences de la première catégorie ne sont pas exploitables actuellement ế qu "il convient d'être circonspect er ce qui concerne certaines essences des autres catégories, celles de la sixième notamment. Nous ne préconiverons dans ce travail que l'exploitation de celles dont la production peut acquérir une certaine importance et dont l'écoulement. en France ou à la colonie même, nous paraît asauré et rémunérateur.

\section{RÉCHNE FORESTIER EN VIGLELR DANS LA COLONE}

\section{RËGLEMENTS D’EXPLOITATION ET TAXES}

Le régime auquel sont solimises les exploitations forestières dans la Colonis et l'exercice des droits d'usage des indigènes onl été fixés par le décret du 18 juin 1912 et par divers arrêtés locaux, dont celui du 23 août 1912 pris pour l'application du décret en question.

Nous ne citerons pas ces textes, la lecture 
pouvant en être fastidieuse. Nous nous bornerons simplement à une analysc assez complèie, mais aussi succincte que possible.

L'Administration locale ne procède pas comme en France, bien que le décret du 18 juin l'y autorise, à l'adjudication de coupes ou parcelles, préalablement aménagées et évaluées par un service forestier. Il lui faudrait en effet comme on procède en Europe, à abattre indistinctement toutes les essences et à ne laisser dcbout que les seuls sujets dont on veuille faire des porte-graines et, en même temps, de futurs arbres d'industrie.

Or, ce n'est pas le cas et ce ne le sera pas avant longtemps encore. On n'exploite guère actuellement, là où on exploite, que 1 à $2 \%$

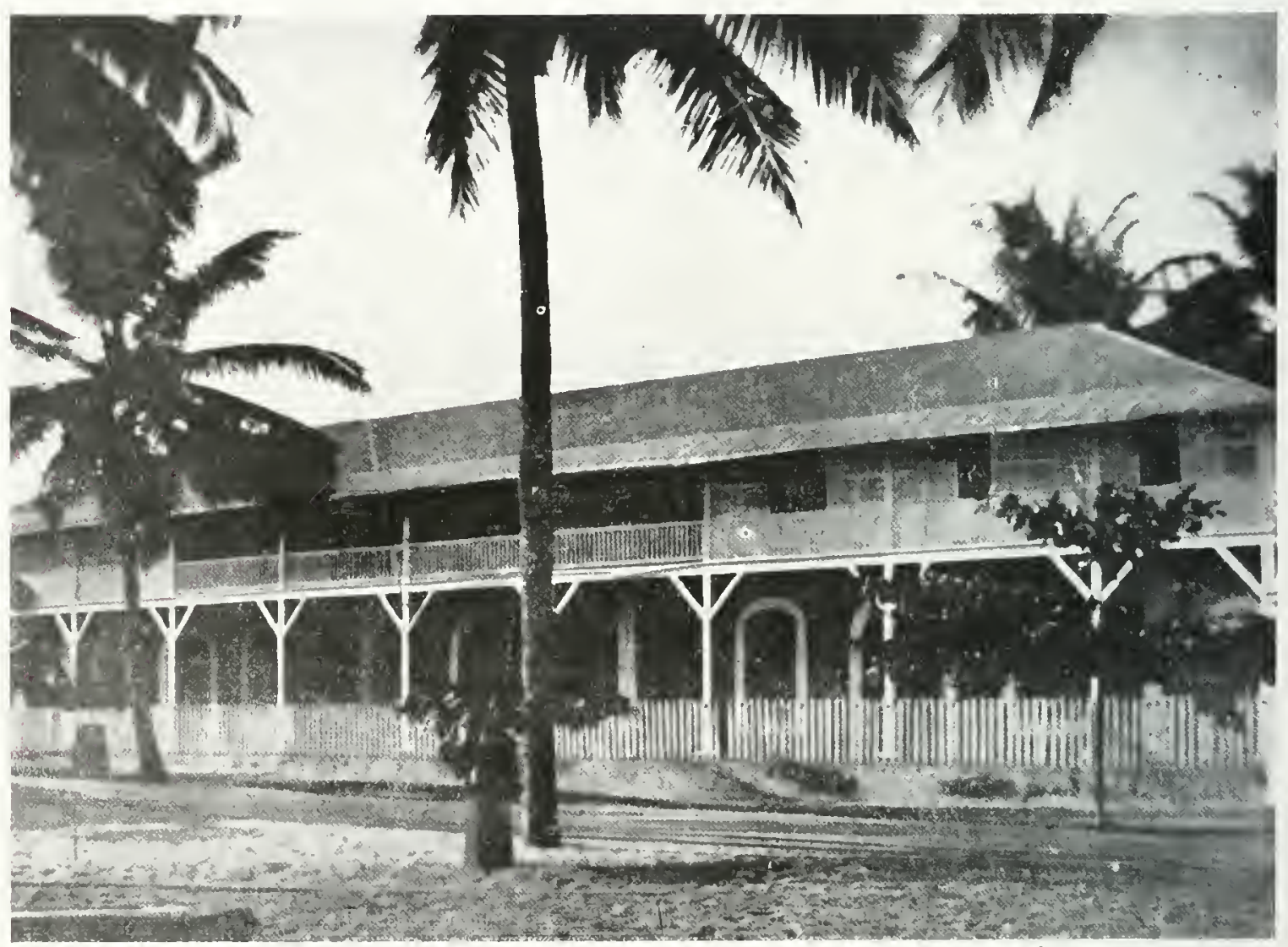

Dueclion du Wharl. - Façade sur le boulevard Treich-Laplene.

Clichè Agencre économique A O. F. .

pour cela une organisation assez coûteuse qui absorberait la majeure partie des recettes provenant des taxes auxquelles sont assujeitis les exploitants. D'autre part, pour qu'une organisation de ce genre pût fonctionner uílement, c'est-à-dire obtenir, sinon l'amélioration de la forêt, tout au moins la conservation de sa valeur aciuelle, il faudrait qu' on puisse obliger les exploitants, adjudicataires ou concessionnaires, à procéder à des coupes rationnelles au maximum de la forêt, soit 2 à $4 \%$ des arbres adultes. On pourrait en exploiter huit ou dix fois autant si l'on se décidait à industrialiser les exploitations, à organiser la coupe et l'écoulement des meilleures esserices. Mais ce n'est que lorsque la Colonie sera pourvae de poris bien outillés qu'il sera possible d'envisager l'utilisation et, par suite, l'abatage de toutes les essences. Les facilités d'embarquement, la réduction des tarifs de fret, permettront, en effet, 
à ce moment, d’installer sur place des scieries à très grand débit pour des bois de charpente à bon marché, et des traverses de chemin de fer: des usines pour la fabrication de pâte à papier, qui absorberont les essences tendres de peu de valeur commerciale; enfin des usines de produits chimiques qui utiliseront, notamment pour obtenir des pyroligneux et des alcools

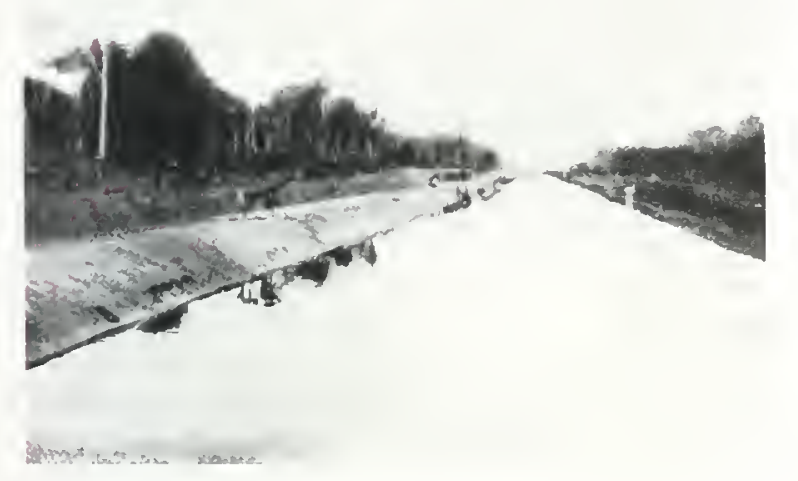

Caral de Kially.

Cliché Agence economique A O.F

méthyliques, tous les déchets, tous les bois impropres aux autres industries.

On pourrait en déduire que le régime actuel conduit à la ruine des peuplements les plus intéressants. Ce serait vrai, sul devait se prolonger trop longtemps. La disparition des essences de choix n'est cependant pas à envisager à brève échéance du fait qu'on coupe les plus gros arbres parmi les meilleurs, les exploitants n'étant autorisés, en effet, à n’abattre, pour les bois précieux surtout, que des sujets ay'ant déjà atteint une certaine dimension. Nous ne voulons pas dire par là que le système soit parfait; il répond en tout cas à une nécessité du moment. Toute mesure prise pour assurer le repeuplement de la forêt en bonnes essences serait superfue tant qu'il ne sera pas possible de contraindre l'exploitant à abattre tous les arbres non réservéz spécialement. Et ce ne sera peut-être que dans vingt ou trente ans quion pourra y penser!

\section{Chantiers}

Au lieu de coupes délimitées par avance. l'Administration locale concède en conséquence des chanticrs ou carrés de forét de 2.500 hectares $(5 \mathrm{~km} . \times 5 \mathrm{~km}$.) dont l'emplacement est choisi par l'exploitant lui-même et dont ce dernier n'exploite que ce qu'il veut durant la période pour laquelle les chantiers sont accordés.

L'octroi, le renouvellement de ces chantiers. l'exploitation dont ils sont l'objet, sont soumis à certaines formalités, à des redevances, dont nous allons donner l'énumération.

\section{Permis d'exploilation}

Tou particulier ou représentant d'une Société désireux de se livrer à l'exploitation forestière ou d'étendre une première exploitation, a la faculté, avant de demander un ou plusieurs chantiers qui entrainent le versement de redevances assez élevées, de faire de la prospection. c'est-à-dire d'étudier une partie de forêt, au point de rue valeur des peuplements ainsi qu'à celui des facilités d'évacuation des bois abattus. Pour que cette prospection lui réserve des droits. il faut qu'il prenne un permis dit d'exploration 1", permis qui peut porier sur une assez grande étendue d'un seul tenant, en zone non ccncédée, et dont les droits sont calculés comme suit :

2 francs par kilomètre carré jusqu’ à 25 kilomètres carrés;

3 francs pour la superficie excédant 25 kilomètres carrés jusqu à 50 kilomètres carrés:

+ francs pour la superficie excédant 50 kilomètres carrés jusqu à 75 kilomètres carrés:

$j$ francs pour la superficie cxcédant 75 kilonètres carrés jusqu'à 100 kilomètres carrés:

6 francs pour la superficie excédant 100 kilomètres carrés.

Une neême personne ou Société ne peut détenir à la fois qu'un seul permis d'exploration. Ce permis est valable pendant trois mois. 11 confère uniquement au titulaire une pricrité pour 
obtenir, durant sa validité, un ou plusieurs permis d'exploitation dans la zone de forêt qu'il concerne.

Les demandes d'exploration doivent être adressées à l'Administrateur du cercle intéressé, appuyées d'un plan ou d'un croquis de la région sollicitée et d'un récépissé constatant le versemeni au Trésor des redevances afférentes. Elles sont transmises au Gouverneur (Service forestier) qui accorde les permis si les zones demandées sont reconnues libres. Si plusieurs demandes sont adressées en même temps pour la même zone. la priorité est accordée à celle qui, la première, a été déposée et enregistrée au Cercle.

Les permis d'exploration ne sont pas renouvelables.

\section{Permis d'exploitation ou chantiers}

Le permis d'exploration n'est pas indispensable pour obtenir un permis d'exploitation ou chantier. De plus, il ne conière pas au tiulaire un privilège absolu sur la zone de forêt qu'il concerne, lorsque cette zone est contiguë à une exploitation en cours et que le chef de celle-ci s'eci acquis des droits sur les parcelles environnantes en construisant, par exemple, une voie Decauville susceptible de les desservir. Il peut donc y avoir opposition de la part de cel exploitant à l'ocíroi des permis demandés dans son voisinage par un tiers. Généralement, ces oppositions sont réglées à l'amiable: à défaut d'entente, elles peuvent être soumises à l'appréciacion du Tribunal de première instance de Grand-Bassam.

Comme nous l'avons dit, le permis d'exploitation ou chantier porte sur une surface de 2.500 heciares, soit 25 kilomètres carrés. Une même personne ou Société peut en obtenir plusieurs, contigus ou non. Ils sont valables pendant une année. Ils peuvent être renouvelés indéfiniment dès l"instanì qu"ils sont, eux ou le groupe de chantiers contigus dont ils peuvent faire partie, l'objet d'un minimum d'exploitation, minimum revisable et fixé par l'Administration.

Les demandes de permis d'exploitation doivent également être adressées au Gouverneur par l'intermédiaire de l'Administrateur du cercle intéressé qui les affiche pendant un mois et les transmet avec son avis motivé. Dès le dépôt au cercle, le chef-lieu est saisi et fait paraitre au Journal officiel de la Colonie un avis indiquant, avec le nom du demandeur, les limites du permis sollicité. Lc délai d'un mois écoulé, et toutes les formalités accomplies, le permis peut être accordé. S̈il y a des oppositions, elles sont réglées comme nous l'avons exposé plus hauí.

Si, d'autre part, le même permis était l'objet de plusieurs demandes et qu'aucun des demandeurs n'ait acquis de priorité par un permis préalable d'exploration ou de droits spéciaux résuliant d'une exploitation voisine, la réglementation forestière autorise l'Administration à mettre ce chantier aux enchères publiques. Le prix d'adjudication tient alors lieu de redevance pour la première année; il ne peut, par suite, être inférieur au taux de cette redevance.

Une demande de chantier entraine le versement d'une somme de 1.250 francs à titre de redevance annuelle et la consignation au Trésor d'une somme de 1.750 francs (1) dont $250 \mathrm{fr}$. pour garantie de délimitation et 1.500 franc; pour cautionnement de garantie du salaire des travailleurs pouvant éventuellement être employés sur le dit chantier. Ce cautionnement peut être constitué par des valeurs ou encore ĉtre remplacé par un engagement souscrit par un autre exploitant ou maison de commerce agréés par l'Administration.

Les récépissés de versement et dépôt, l'engagement tenant lieu de cautionnement, le cas échéani, doivent être annexés à la demande établie en triple expédition. Doivent également

(1) Tous les permis sont, en outre, soumis à la formalité du timbre $(0,20$ des redevances, plus 0,50 taxe fixe pour la copie). Les cautions sont timbrées à $0,50 \%$. 


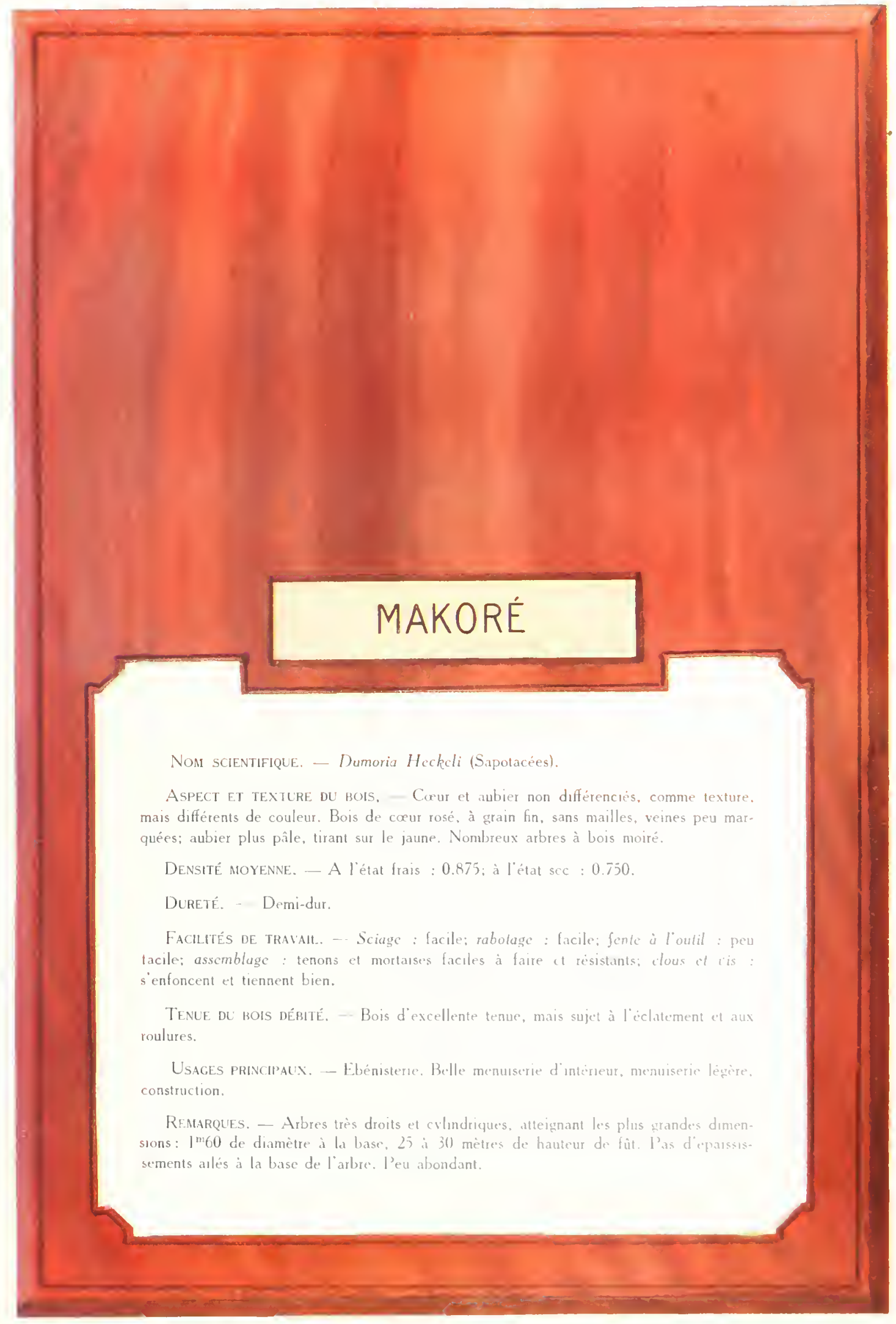



ctre joint. trois exemplaires du plan du chantier lequel plan doit avoir pour base une voie d'eau, une voie ferrée, une route ou un sentier faciles à repérer: ce plan doit être rattaché à un point géographique connu.

\section{Base des chantiers}

Bien que le permis d'exploitation soit en principe un carré parfait, la base sur laquelle il esi construit peut n'être pas rectiligne. Dans ce cas, on construit le carré sur les points extrêmes de la base, ces points étant reliés par une ligne droite imaginaire et, suivant les cas, les superficies englobées par les méandres de la bazo vraie, s'ajouient au permis ou en sont retranchées.

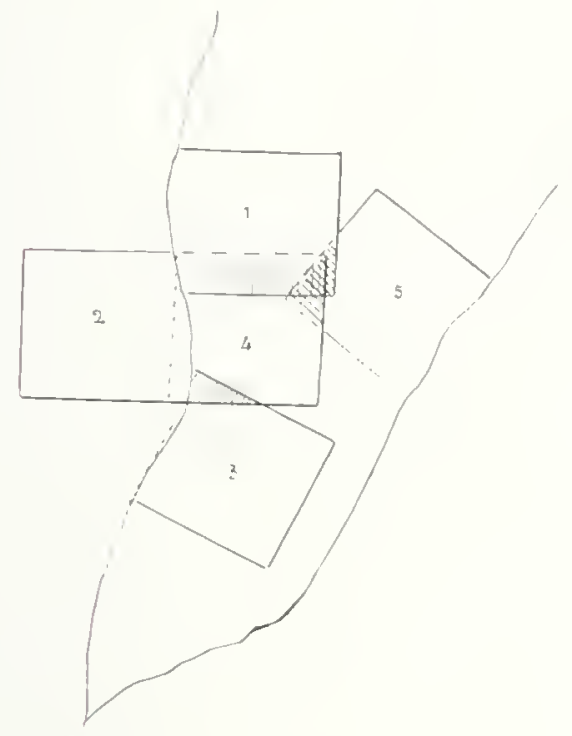

Observation. C'est la rivic̀re et non le pointillé yui sépare le chantier n" 2 du chantier n" 4 .

Les n" I et 3. les premiers accordés, peuvent être exploités sur toute leur superficie.

Le chantier n" 4 , par contre, est très restreint. Son litulaire ne pourra exploiter dans les parties hachurées du 1 et du 3 que lorsque ces derniers chantiers seront périmés. Il en est de même pcur le chantier 5 qui ne pourra être exploité sur la partie hachurée en lignes horizontales qu'après que seront périmés les n' 1 et 4 .

\section{Cheiauchement de chantiers}

Il arrive fréquemment, d'alitre part, que des permis contigus, mais dont la base est inueuse. comme c'est généralement le cas lorsqu il s'agit de rivières, chevauchent les uns sur les autres. Lo premier permis accordé a, dans ce cas. la priorité sur le second, et le sccond sur les suivants pour la partie de forêt qui est comprise dans plusieurs chantiers.

Le dessin ci-contre fora mieux comprendre que de trop longues explications.

\section{Chantiers en bordure de la voie ferrée}

Les chantiers limitrophes de la voie ferrée sont l'objet d'une délimitation spéciale. Pour éviter des chevauchements et permettre néanmoins le plus d'exploitations possibles, l'arrêté du 23 août 1912 a précisé en effet que les limites Nord et Sud de ces chantiers seraient non des perpendiculaires à la voie, mais des lignes Est-Ouest, partant des points kilométriques $j$ ou multiples de 5. Comme la ligne du chemin de fer suit une direction générale S.E.-N.O., il s'ensuit que la plupart des chantiers accordés en bordure ne sont pas des cariés, mais des trapèzes dont la superficie est plus ou moins inférieure à celle des autres chantiers.

Demandes de permis visant une zone dont la concession va arriver à expiration.

Il arrive que, pour une raison quelconque. le détenteur d'un chantier abandonne celui-ci avant d'en avoir achevé l'exploitation, quelquefois mème avant de l'avoir entreprise. La demande de renouvellement n'étant pas faite ou n'étant pas suivie d'effet, le chantier va redevenir libre: deux ou plusieur: compétiteurs le sollicitent, soit comme permis d'exploration. soit comme permis d'exploitation, à des dates différentes. mais avant l'échéance do validité du permis antérieurement accordé. Dans ce cas. la date de dépôt au cercle intéressé ne conlérera de priorité à l'un des intéressés que s’il n’est déposé que des demandes d'exploration. S’il y a en même temps une demande d'exploitation. elle prime les premières. mène si déposée après 
clles: sil y en avait deux ou plusieurs, l'Administration procéderait, comme il a été déjà dit, par voie d'adjudication.

\section{Les permis sont personnels}

Les permis d'exploitation ou d'exploitation de forêt accordés sont strictement personnels. Ils ne peuvent être transférés à un autre titulaire ou

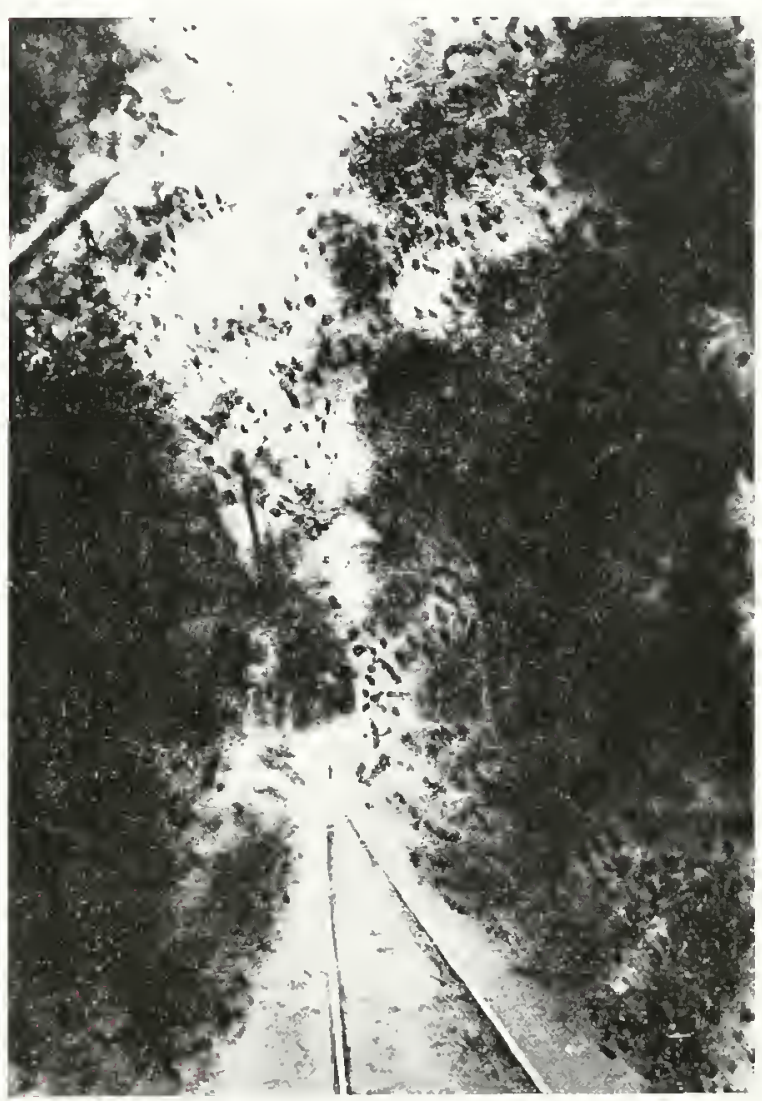

$$
\begin{aligned}
& \text { En foret. - Voie Decauville. } \\
& \text { Chiche Métayer. Grand.Bassam }
\end{aligned}
$$

apportés à une Société qu'avec l'autorisation du Gouverneur en Conseil d'Administration. En fait, ces transferts ne sont jamais refusés s̈ils ne cachent pas un but spéculatif évident.

\section{Règlements d'exploitation. Taxes.}

L'exploitation des chantiers, leur renouvellement, sont soumis à des règles précises. Le détenteur est tenu :
I De tenir un carnet de chantier sur lequel il inscrit très régulièremení tous les arbres abattus, le nombre, les dimensions et le numéro des billes fournies par chacun d'eux, la date de leur sortie du chantier. Ce carnet, qui sert de base à la perception des taxes et au contrôle des coupes, doit être très bien tenu; il doit être communiqué à l'Administration chaque fois qu'elle en fait la demande;

$2^{\circ}$ De ne faire voyager les billes obtenues. jusqu'au port d'embarquement, qu'accompagnées d'extraits de carnets établis en triple expédition et visés par l'Administraieur du ccrcle dans lequel se trouve le chantier.

Les exploitants qui ne tiendiaient pas comptc de ces formalités, et qui seraient convaincus ou suspectés d'avoir sorti frauduleusement des billes de leurs chantiers, pourraient être condamnés à des peines sévères; leurs bois pourraient être saisis et confisqués;

3" D'acquitter une taxe dite d'abatage, dont nous indiquons plus loin le taux pour chaque essence, et qui varie de $\mathrm{I}$ à 15 francs par mètre cube (1) de bois abattu, sorti ou non du chantier. Cette taxe est payable a's moment du visa par l'Administration, des extraits de carnets, c'est-à-dire à la sortie des billes du chantier. Pour les billes abandonnées, elle est payable au moment du renouvellement du chantier ou de son expiration, lorsque le carnet doit être envoyé au Service forestier pour vérification et contrôle des coupes. La mention de payement de la taxe (2) doit ètre indiquée sur les extraits et sur le carnet lui-même;

4. De payer une taxe de repeuplement de 10 francs par arbre abattu (A cajou, Tiama et Iroko seulement) ou de replanter effectivement. dans des terrains qui lui sont concédés à cet effet et doni il peut devenir propriétaire, un nombre d'arbres quintuple de celui abattu et

(1) Cette taxe vient d'être modifiée (V. p. 39.).

(2) Cette taxe n'a été instituée que par arrêté en date du 7 octobre 1920. Le taux devrait en être revisé assez fréquemment, suivant les cours auxquels sont vendus les bois sur place. 
d'essences déterminées, au nombre desquelles les bois précieux, le cacaoyer et le palmier à huile;

5. De sortir annuellement de son chantier pour en obtenir le renouvellement, un minimum de 300 tonnes de bois, quelles que soient les essences exploitées. (Lorsqu'un même exploitant a plusieurs chantiers qui sont contigus, i] n'est pas obligé de les exploiter tous, pourvu qu'il sorte de l'ensemble un tonnage en rapport avec le nombre de chantiers).

Cette mesure, prise en vue d'éviter l'accaparement de chantiers, n'est pas toujours appliquée à la lettre. Tout d'abord, il est admis que l'installation d'une voie Decauville peut tenir lieu d'exploitation effective pendant un et même deux ans. Ensuite, il est des cas de force majeure, maladie, accidents, insuffisance de crue dans les rivières, mévente des bois. comme en 1921, etc. L'Administration locale examine toujours avec beaucoup de bienveillance les cas particuliers qui lui sont soumis.

\section{Renouvellement des chantiers}

Le renouvellement des chantiers est accordé par le Gouverneur dans les mêmes conditions que les premiers permis, sauf que le péititionnaire n'a pas à fournir (en dehors d'un récépissé de versement d'une nouvelle somme de 1.250 francs comme redevance annuelle) de cautionnements, plans, etc. Par contre, il doit justifier du tonnage sorti du chantier à renouveler, tage en envoyant son carnet de chantier, acquitter enfin la taxe dite de repeuplement sil ne fait pas de déclaration concernant le repeuplement en nature.

Il doit aviser l'Administration de son intention de faire renouveler son permis un mois au moins avant l'expiration de celui-ci.

\section{Repeuplement en nature}

Les plantations faites par les exploitants pour tenir lieu de la taxe de repeuplement sont soumises au contrôle de l'Administration. Elles doivent être entretenues pendant trois ans au moins, faute de quoi l'Administration pourrait les reconnaitre comme sans valeur et réclamer aux exploitanis les taxes qu ils auraient normalement dû acquitter.

\section{Fraudes}

Les coupes irrégulières, celles qui sont faites en dehors des chantiers, les tentatives de dissimulaiion d'arbres abattus, de tromperie sur la quantité de bois sortis, etc., sont punies très sévèrement. Faute par l'intéressé, lorsqu'un délit a éié constaté à sa charge, de demander et

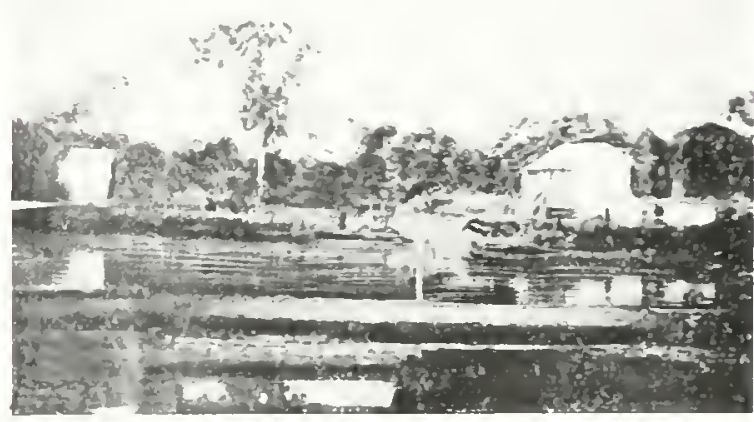

Debarcadere d'Ono.

Cliché Metayer Grand.Bassam

accepter un règlement transactionnel. l'Administration saisit des faits le Procureur de la République qui, après instruction, traduit l'intéressé devant le Tribunal correctionnel de GrandBassam. Le décret du 18 juin prévoit des pénalités rigoureuses, jusqu'à 10.000 francs d'amende, la confiscation des bois, la révocation des permis et la suspension pendant cing ans de tous droits à l'attribution de nouveaux chantiers.

\section{Grandes concessions}

Il ñy a là, à la Cóte d"lvoire, qu'une seule concession qui mérite cette appellation, celle de la Compagnie forestière de l'Afrique Fran- 
çaise, accordée en 1911. Cette Société jouit de privilèges spéciaux antérieurs à la réglementation intervenue en 1912. D'autres Sociétés détiennent des superficies de forêts aussi considérables (60.000 hectares), mais sous forme de chantiers de 2.500 hectares, qu'elles renouvellent au fur et à mesure de l'échéance des délais de validité.

Le Gouvernemeni local a étudié de nouveau la possibilité d'accorder, pour vingt ou trente années, des concessions de grande étendue aux Sociétés disposées à engager des capitaux im-

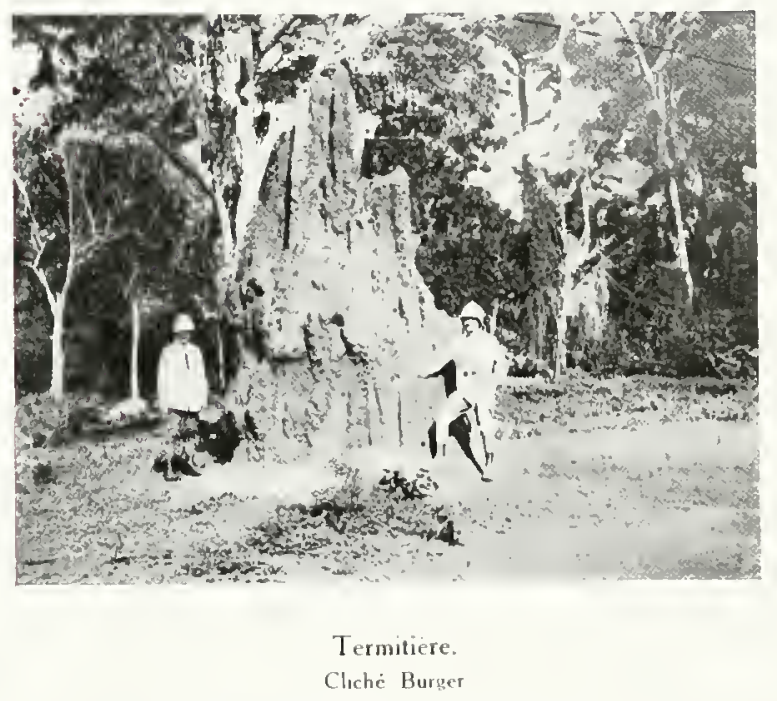

portants dans la Colonie. Un cahier des charges type a été établi dans ce but. Les bénéficiaires seraient toutefois astreints au: mêmes obligations que les détenteurs de chantiers ordinaires, en ce qui concerne les redevances eî le tonnage minimum à sortir chaque année par 2.500 hectares concédés. La question a été soumise au Ministre des Colonies. Elle est de nature à intéresser les personnes ou groupements désireux de placer des capitaux dans les affaires coloniales.

VALEUR RESPECTIVE DES ZONES DE FORÊTS AU POINT DE VUE DE LEUR EXPLOITATION

Toute la forêt de la Côte d'Ivoire n'est pas susceptible de pouvoir être mise en exploitation immédiatement ou dans un délai rapproché.
En admettant, ce qui ne pourra d'ailleurs se réaliser que dans une période assez lointaine, que peuplements pauvres ou riches de la région côiière puissent être exploités à tout venant, des industries locales arrivant à tỉ̈er parti d’à peu près toutes les essences, un facteur essentiel interviendra toujours pour rendre impossible, en fait, l'exploitation des zones éloignées de iout moyen pratique ei peu coûteux de communication, c'est le facteur transport. Les bois précieux peuvent à la rigueur être coupés assez loin dans l'intérieur, le prix auquel ils sont réalisés permettant de couvrir des frais de transport élevés. Mais dès qu'on envisage la question au point de vue des bois de charpente et de menuiserie, la zone exploitable se rétrécit singulièrement. Elle se rétrécit plus encore pour les bois à traverses de chemin de fer, et quand on voudra exploiter les essences propres seulement à la pâte à papier ou à la fabrication de produits chimiques, il ne faudra guère s'écarter des lagunes, rivières ou fleuves navigables ou flottables, les industries de transformation auxquelles nous faisons allusion n'étant viables qu'autant que la matière première, c'est-à-dire le bois, leur reviendra à un prix extrêmement bas.

Actuellement, il y a déjà environ six cent cinquante mille hectares de concédés à des exploitants. Bien eniendu, ces six cent cinquante mille hectares représentent, sinon ce qu'il y a de plus riche en $A$ cajou, tout au moins ce qu'il y a de plus facile à exploiter. La majeure partie des chantiers se trouve en arrière de GrandBassam, soit en bordure de la voie ferrée partant d'Abidjan, $1^{\text {re }}, 2^{\circ}$ et $3^{\prime}$ zones, soit en bordure des voies navigables ou flottables, fleuves, rivières, lagunes. Les autres chaniiers se trouvent en arrière d'Assinie, de GrandLahou ou de Sassandra.

Les chantiers bien placés de la zone en arrière de Bassam sont de beaucoup les plus favorisés; les bois sortis du voisinage du chemin de fer, des lagunes Ebrié et M'Poiou, du Comoë, peuvent être évacués à peu de frais et 


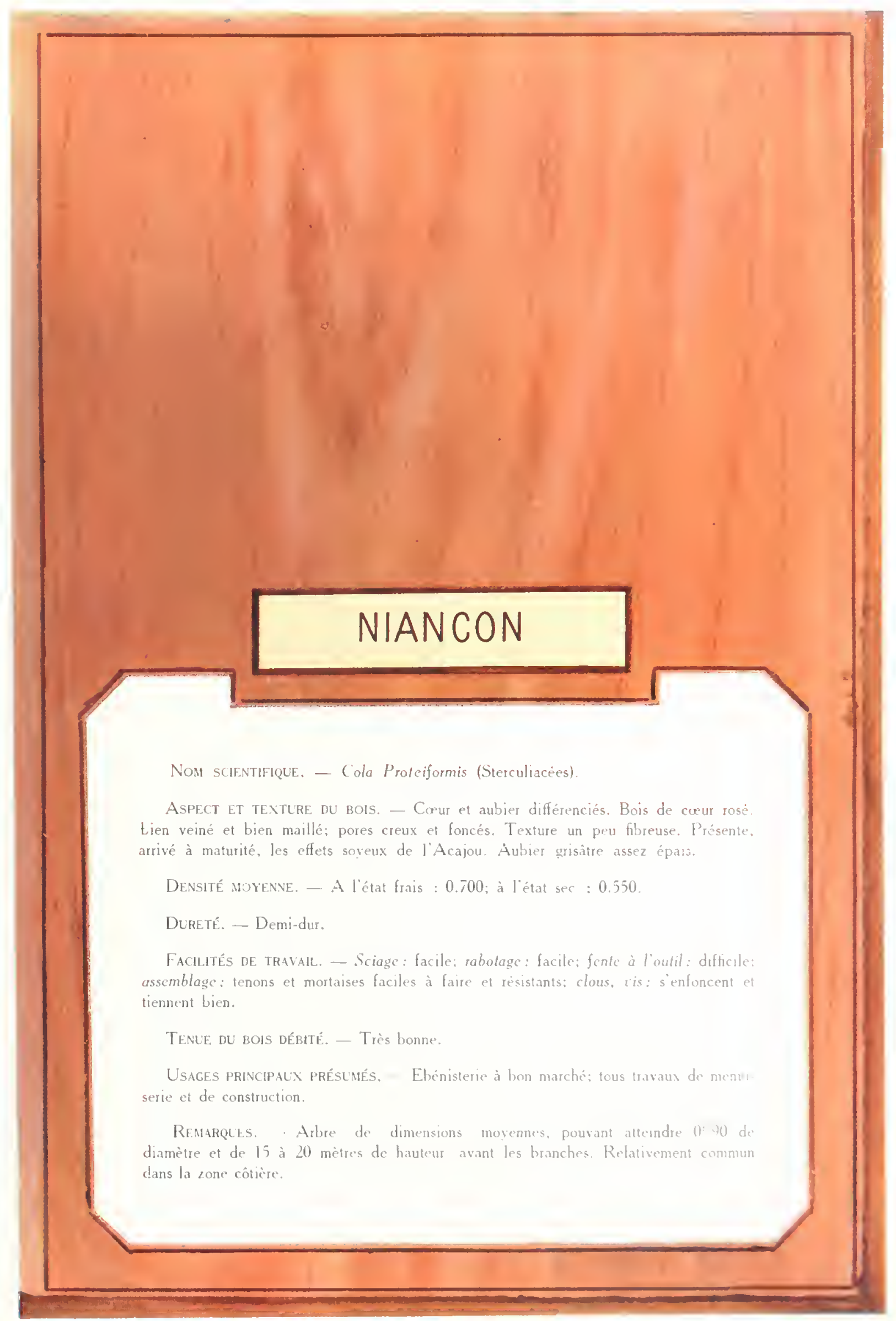





\section{TABLEAU ANNEXÉ A LARRETE DU 7 OCTOBRE 1920}

portant creation dune Taxe d'abatage

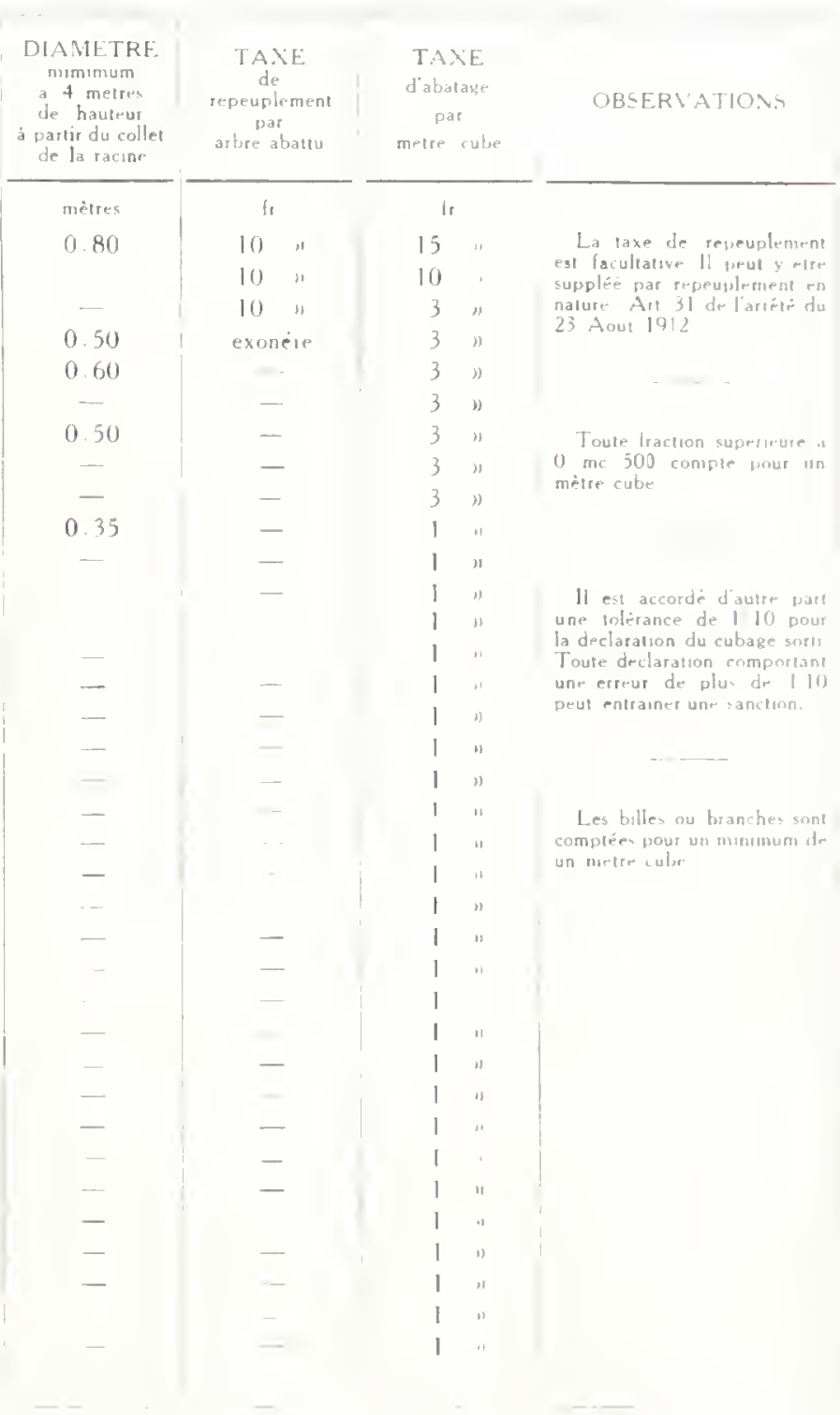

Acajou (Kuhya ivorensis)

Iroko (Chlorophira excelsa)

Tiama (Entandrophragma sp.)

Badi (Surcocephalus Pobeguini)

Bosse (Trichilia cedrata)

Makore (Dumoria Heckeli)

Niangon (Cola proteiformis)

Rikio (Uapaca bingervillensis)

Framire (Terminalio ivarensis)

Aielé (Canariun occidentatis)

Apome (indelermine)

Azobe (Lophira procera)

Ako (Antiaris lovicaria)

Asas (Bridelis speciosa)

Aninguery (Malencantha robusta)

Abale (Petersia vridiflora)

Adjansi (Cicca discoideus)

Adjouaba (Hamaloaluphis Barleri).

Anioukeli (Pachypedanthium)

Avodie (Bingera africana)

Bahia (Mitragyne macrophylla)

Bodioa (Pyncertia occidentalis)

Coula (Coula edulis)

Dabema (Piptadenia africana)

Faro (indetermine).

Frake (Terminalia allissima)

Kroma (Klainedoxu).

Lo (Parkia agboensis).

Oboto (Ochrocarpus africana ou Manmea africana).

Olon (Fragara macrophilla)

Pri (Funtunia africana)

Sougue (Parinarium termifolium)

Samba (Triplochyton snleroxylon)

Senan (Massebotrya stufiana)

Sibo (Sarcocephalus esculantus)

Tali (Erytroploeum guineense)
Nola. - Ainsi que nous l'avons signalé plus haut. les taxes prévues à l'arrête ci-dessus viennent d'être modifiées. Un arrêté du Gouverneur de la Colonie, en date du 19 décembre 1921, précise (uue les nouvelles taxes. calculées sur le nombre d'arbres abattus el non sur le volume de ces arbres, ce qui en rendra la perception plus facile, sont fixées comme suit:

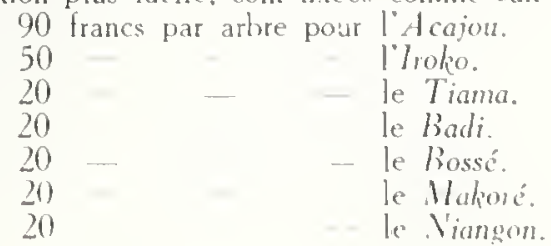

20 francs par arbre pour le Rikio. 20 le Framiré.

6 - - - les autres essences

Ces nouvelles taves correspondent approvmativement aux anciennes pour l"itcalou et l'Thoko. ansi que pour le Badi. le Bossé et le Makors. Eilles sont moins lourdes pour le Tiama. Par contre. elles paraissent trop élevées pour le Viangon, le Rikio e' le Fanire, arbres d'asser petit diametre", qui ne domnent guère que de 2 à 4 metres cubes en bois exploitable.

La tave de repeuplement nest pas modifice. De mime sont maintenus les diametres minima au-dessous desquels ne peurent étre ahattus les differentes essences inumerces i lourrete du 7 octohre 10? 
pendant toute l'année sur Grand-Bassam d'où. à la faveur du wharf, ils sont expédiés facilement par les cargos de passage. Lez chantiers siuués en deuxième ou troisième zone exigent déjà, pour l'évacuation des bois, des voies Decauville coûteuses, une main-d'œuvre plus abondante. Ceux qui sont desservis seulement par les petites rivières sont encore moins ayan.

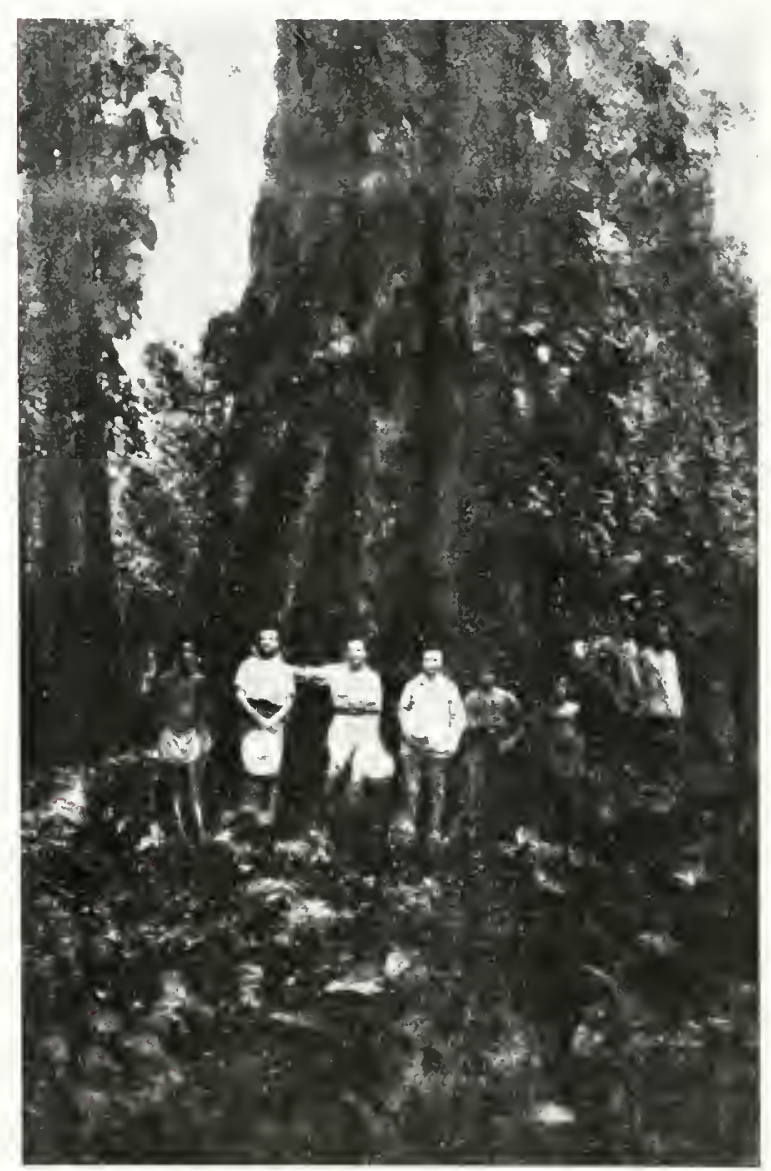

Un Tiama-tiama de $7^{m} 50$ de diametre. Cliche Métayer - Grand.Bassam

tagés, car l'évacuation des bois coupés sur ces chantiers est subordonnée à la régularité des crues périodiques; si ces crues ne se produisent pas ou sont insuffisantes, les billes restent en panne, pourrissent ou se fendent en attendant la crue suivante qui leur permettra peut-être d'atteindre le port d'embarquement. C'est une campagne perdue pour l'exploitant, quelquefois la ruine.
Dans les régions d'Assinie, Grand-Lahou et Sassandra, les exploitants, quels que bien placés et bien desservis que fuissent être leurs chantiers, doivent compter avec les aléas des expéditions, les cargos trarsporteurs mettant plus ou moins de complaisance à toucher ces points et la barre rendant toujours plus ou moins difficiles les opérations de chargement.

Ces 650.000 hectares concédés représentent donc incontestablement le meilleur de la forêt au point de vue exploitation de l'A cajou et des essences d'ébénisterie. Si l'on a déjà coupé depuis dix ans (tant sur les chantiers en cours de validité que sur les chantiers voisins abandonnés depuis et qu'on peut évaluer à 100.000 hectares environ) 300 à 400.000 tonnes d'Acajou et une cinquantaine de mille tout au plus d'autres essences comme: le Tiama, l'Iroko, le Bossé, etc., ces 650.000 hectares sont loin d'être exploités complètement pour le seul $A$ cajou. Ils sont à peu près vierges de toute exploitation en ce qui concerne les auires essences d'ébénisterie; ils le sont complètement (exception faite pour quelques chantiers qui servent à alimenter les scieries locales) en ce qui concerne les bois de menuiserie et de charpente.

Pour l'Acajou, la zone non exploitée accessible aux coupeurs est encore assez vaste, surtout si les cours de cette essence se maintiennent élevés et permettent l'installation de chemins de fer Decauville de 15 à 25 kilomètres, reliant les concessions aux voies naturelles d'évacuation. Pour les bois de valeur̃ moindre, il ne faut pas songer à aller aussi loin dans l'intérieur. Néanmoins, leur zone d'exploitation est relativement étendue, car elle comprend, en dehors de la majeure partie de la zone actuellement exploitée pour l'Acajou, toute la partie de forêt qui est en bordure des lagunes ou de la mer et qui n'a jamais été exploitée parce que ne contenant pas ou presque pas d'Acajou.

Pour nous résumer, nous dirons que la zone exploitable pour l'Acajou et les meilleures essences comprend, au maximum, en l'état actuel des choses, 1.200 à 1.400 .000 hec- 
tares (1). Ce chiffre est à ramener à 300 . 400.000 pour les bois de menuiserie et de charpente de choix susceptibles de flotter. Pour les essences de moindre valeur et les essences lourdes, il faudra réduire encore sensiblement ce dernier chiffre et, si l'on envisage pour plus tard la coupe des bois à tout venant, pour l'alimentation d'industries locales, il ne faut guère compter, pour ce genre d'exploitation, que sur 200 à 250.000 hectares. C'est déjà quelque chose, car ces deux cent mille hectares pourraient fournir, en bois tendres, par des

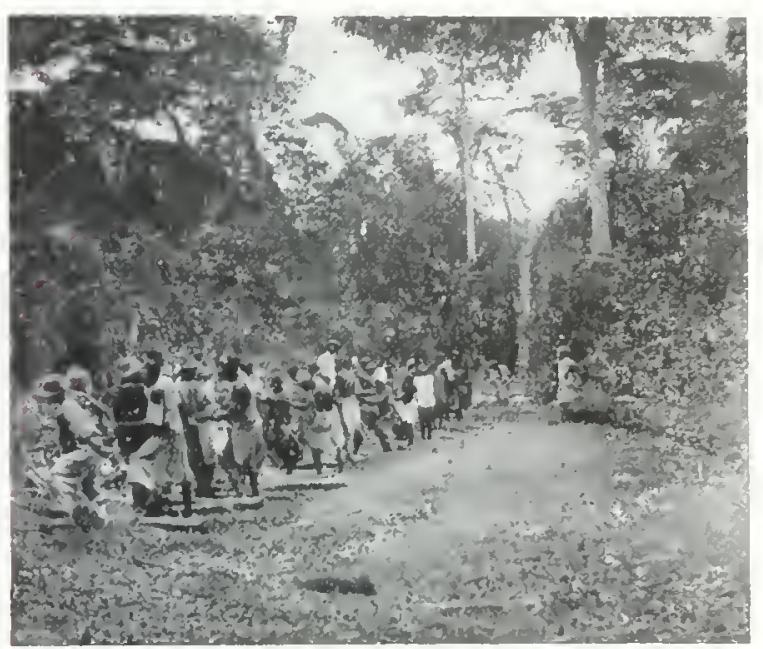

Tirage de billes en foret.

coupes réparties sur quarante années, plus de 500.000 mètres cubes de bois par an (2).

Nous sommes donc loin de l'exploitation totale des 12.000.000 d'hectares de forêt de la Colonie. Plus tard, évidemment, par l’aménagement des voies fluviales ou la construction de nouvelles voies ferrées partant de la côte, il sera sans doute possiblc d'étendre peu à peu

(1) Si l'on tient compte des surfaces défrichées. encore utilisées par les indigènes, ou réoccupées par une forêt de valeur secondaire.

(2) Le $\mathrm{C}^{t}$ Bertin estime ì $250 \mathrm{~m}^{3}$ au minimum par hectare les peuplements de la grande foret. Nous prenons un chiffre beaucoup plus faible pour la zone voisine des lagunes et des fleuves navigables où abondent les parties peu ou mat boisées. savanes, palmeraies. terrains cultivés. etc. les superficies exploitables. Cette éventualité. en tout cas, est trop éloignée de nous pour que nous songions à en envisager les conséquences.

\section{MOYens et MODES D'ÉVACUATION SLR LA COTE DES BOIS ABATTUS. - ENTREPOTS. - ExPÉDITIONS}

\section{Tirage à main d'hommes.}

Quelle que soit la situation des chantiers, qu'ils soient desservis par un simple torrent d'hivernage, par une voie d'eau plus importante, par une voie Decauville, voire même par le chemin de fer à voie d'un mètre qui aboutit à Abidjan, il y a toujours une certaine distance entre le point où a été abattu l'arbre. et celui où les billes seront mises à l'eau pour être "flottées " ou chargées sur wagonnet ou wagon. Cette distance peut varier de quelques dizaines de mètres à dəux, trois, cinq kilomètres et même plus, suivant les chantiers et la nature du sol, suivant l'outillage des exploitants qui, sils disposent de rail Decauville, peuvent établir des embranchements plus ou moins rapprochés.

Or, pour amener les billes au point où le wagon les prendra, on n’a usé jusqu'à présent que du procédé le plus primitif : le tirage de ces billes à main d'homme. Certes, dans un pays où les animaux de bât ou de trait ne vivent pas (1), c'est, sinon le seul dont on puisse disposer, du moins le plus économique, tant qu'il ne s'applique qu'à de très courts trajets. Dans une forêt dont on n'exploite guère qu' un arbre en moyenne à l'hectare, et cette movenne n'est pas toujours atteinte par les ccupeurs d'Acajon. il n'est pas possible de conduire une voie Decauville au pied de tous les troncs abattus. Cela entraincrait à des frais excessivement élevés.

L'usage de treuils à rapeur ne serait guère plus pratique, étant donné que ces appareils ne peuvent guère fonctionner que sur des lignes droites, difficiles à tracer en forêt.

(1) Par suite des trupanosoniases. 
Quoi qu'il en soit, le tirage par la traction humaine demanderait à être réglementé et tolérẻ seulement pour de courtes distances, chaque exploitant étant mis en demeure de placer des voies Decauville sur son chantier.

Peu à peu des moyens moins primitifs, tracteurs légers, par exemple, viendront remplacer I'homme pour ce genre de travail. Du reste. au fur et à mesure que se développera l'exploitation forestière, qu'augmentera le nombre des essences exploitées, par suite le tonnage sorti des chantiers, les exploitants auront avantage à utiliser du Decauville, à multiplier les ramifications volantes, à réduire au minimum la traction humaine, la main-d'œuvre, déjà peu abondante, étant mieux utilisée à d'autres emplois : abatage, tronçonnage, équarrissage des bois, ouverture de chemins, chargement des billes, eic.

Comment s'opère le tirage? Sur des chemins tortueux, tracés entre les gros arbres et sur lesquels on a placé, en travers, des rondins de bois qui permeitent aux billes placées sur une sorte de traîneau ou de schlitt, de glisser sous l'effort de la traction. Celle-ci s'effectue au moyen d'un filin accroché ou attaché à l'avant de la bille: $40,50,60$ hommes, et quelquefois plus, s'attellent deux par deux à des fiches en bois passées dans ce filin et entraînent la bille avec eux dans leur mouvement en avant. Très souvent, on place également un filin ou une corde à l'arrière avec quelques hommes dont la mission est de surveiller la direction suivie, faire éviter les écueils, ralentir la marche et éviter lez accidents dans les descentes brusques, etc.

50 à 60 hommes arrivent à tirer une bille de 2 tonnes lorsque le trajet à accomplir cst assez. court. Lorsque la distance atteint et dépasse 2 kilomètres, il faut renforcer l'équipe : 70 hommes ne sont pas de trop. Pour les grosses billes qui font jusqu'à 6 tonnes, on met jusqu'à 100 et 120 hommes; l'opération est nécessairement très lente.

Une bonne équipe de 60 hommes peut faire parcourir à une bille d'un poids moyen de 2 tonnes, de 6 à 10 kilomètres par jour, suivant que le chemin de tirage est plus ou moins accidenté, plus ou moins bien fait et entretenu. C'est dire que, lorsque le trajet à accomplir n’est en moyenne que de 600 à 800 mètres, cette même équipe peut rassembler 8 à 10 billes dans la même journée.

Les billes rassemblées près d'une voie Decauville sont chargées, soit qu'elles aient été équarries, soit qu'elles soient restées en rondins, sur des wagons-plateforme. Deux wagons par bille; sauf pour les petites, pour lesquelles un seul peut suffire.

Sauf quelques rares exceptions, lorsqu'il s'agit de grosses exploitations ef de voies posées pour longtemps, ces chemins de fer Decauville sont construits le plus économiquement possible, sans ballast; les rails, montés sur des traverses métalliques, qu'on double par des rondins de bois si elles ne sont pas assez rapprochées, sont simplement posés sur le sol. Le rail le plus communément employé est celui de $9 \mathrm{~kg} .500$ au mètre. Les éléments de voie sont généralement de 5 mètres avec 4 cu 5 traverses. On emploie peu d'éléments courbes, malgré la sinuosité des tracés; quelques aiguilles sont nécessaires pour les embranchements.

Il n'est guère que deux Sociétés qui emploient du matériel plus fort et se servent de locomotives pour la traction des wagonnets. Partout ailleurs, ceux-ci son: poussés par des hommes. Sauf dans les côtes accentuées où elles doivent être renforcées, des équipes de 12 à 15 hommes peuvent faire avancer des billes de poids moyen à une vitesse de 3 à 4 kilomètres à l'heure. C'est déjà une économie sérieuse de main-d' cuvre sur le tirage des billes par schlittage.

\section{Flottage des bois en rivières et en lagunes.}

Le flottage des bois abattus, là où il est possible, constitue incontestablement le procédé d'évacuation le plus avantageux. Nous avons vu que le système fluvial de la colonie laisse malheureusement beaucoup à désirer, soit que 


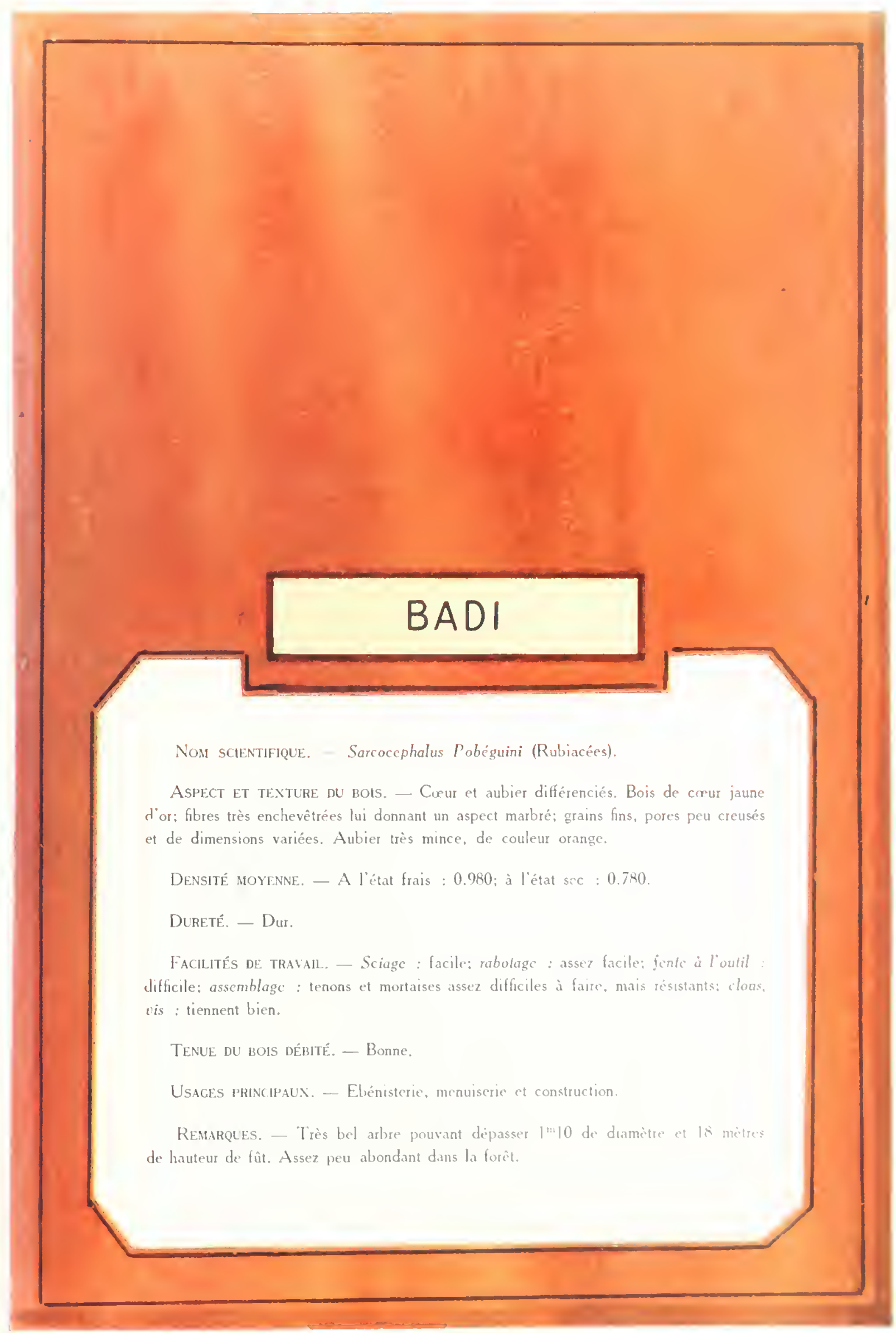



les rivières soient rendues impraticables par les rapides. soit qu'elles ne coulent vraiment qu'à certaines époques de l'année, aux saisons de pluie. Les voies qui marchent, et qui marchent d'une façon permanente, sont donc relativement peu nombreuses. Il n'est guère, en dehors des lagunes, dans la zone exploitable de forêt, que le Comoë jusqu’à hauteur d'Alépé, le jours des crues sulfisantes pour permettre la descente des bois. D'autres, torrentueuses, sortent de leur lit dès que montent les eaux et s'écartent quelquefois à plusieurs kilomètres dans la forêt. Combien de billes d'A cajou sont pourries le long de ces rivières capricieuses. soit qu'elles n'aient pu être évacuées faute de crue suffisante, soit qu'elles aient été entrainées dans

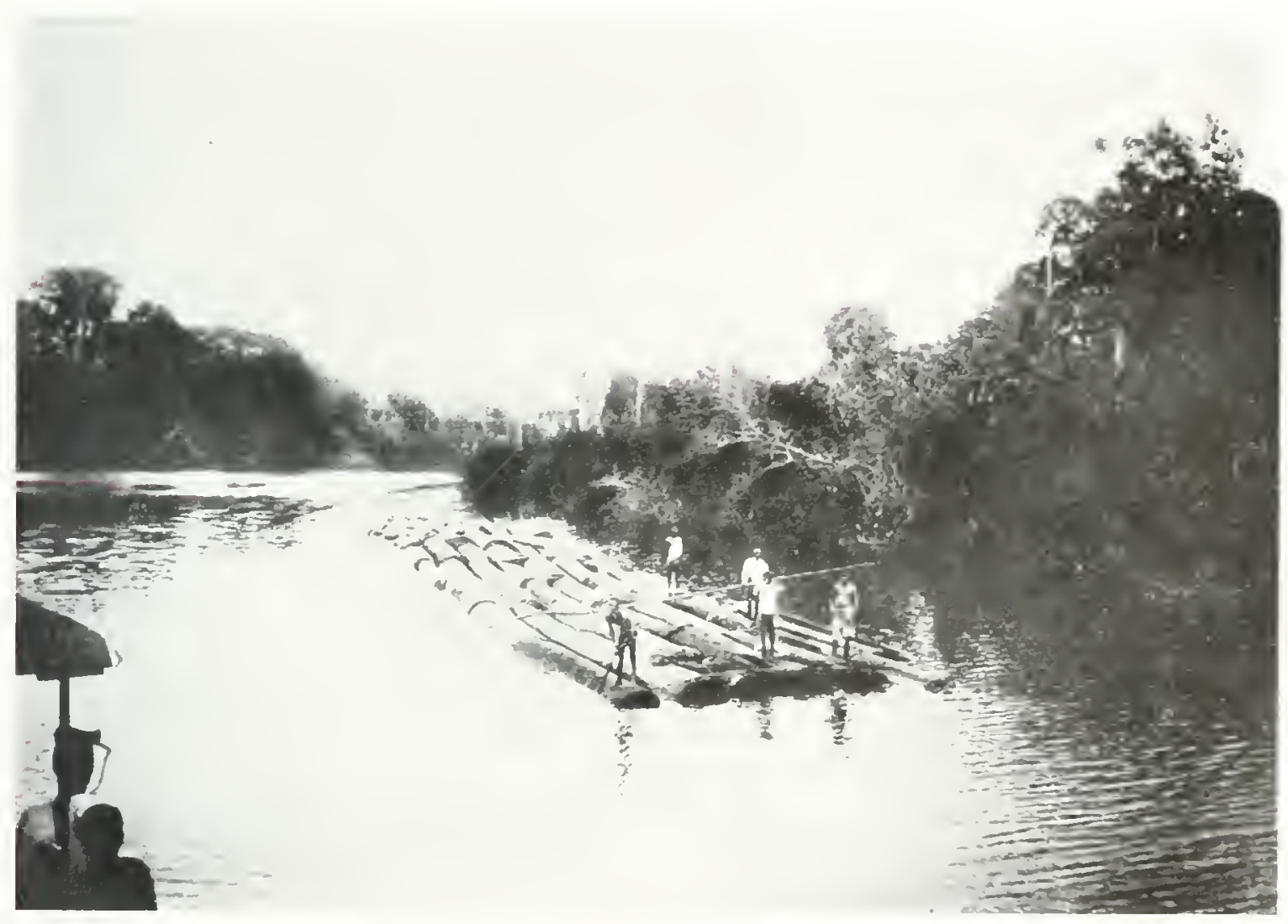

Au fil de l'eau. - Drome descendant ver Basatam

Cliche Metaver - Grand Bassara

Bandama jusqu'à hauteur de Broubrou et le Sassandra jusqu'au confluent de la rivière Davo (cette rivière elle-même est flottable une bonne partie de l'année sur une vingtaine de kilomètres) qui méritent cette appellation. La période de flottabilité des autres cours d'eau varie de huit jours à deux mois par an (1), suivant leur importance et suivant l'abondance des pluies tombées. Certaines rivières n'ont pas tou-

(1) Entre le 15 mai et le 15 juillet et en octobre. la forêt voisine et égarées sous des amas de broussailles.

Sur les petites ririères, dès qu approche la période de crue, on met les billes à l’eau. 11 ne faut pas perdre un jour. Les eaux montent. les billes partent les unes à la suite des autres. escortées par des équipes de manœurres en pirogues ou perchés sur les billes mêmies. qui ont misison de les surveiller. de les diriser. d'aider au passage des seuils. Si la protondeur et le courant sont favorables et si la distance à 
parcourir pour arriver à l'endroit à partir duquel la voie est meilleure n'esî pas trop grande, la descente se fait assez facilement avant que le niveau des eaux ait trop baissé. Sinon, il faut attendre six mois ou un an à ur point quelconque

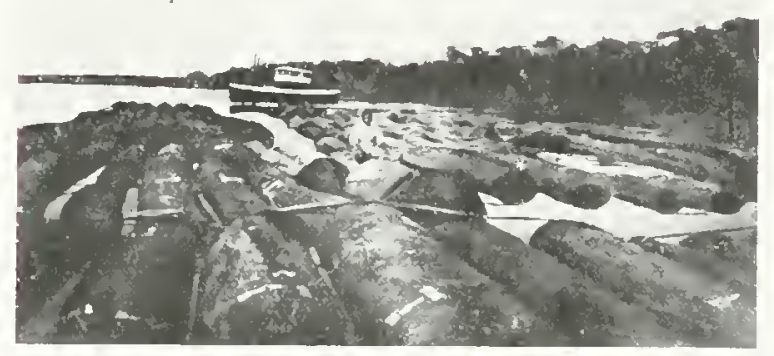

Dròme d'acajou, - Le remorqueur "Le Comoe", de la C" Kong. Cliché Métayer - Grand-Bassam

la crue suivante. Tout n'est pas rose dans le métier de "coupeur de bois "! Nous en avons connu qui n'ont pas fait fortune et qui, pourtant, ont payé de leur personne jusqu'à rester des journées entières dans I'eau à pousser euxmêmes leurs billes, à aider leurs hommes à les soulever aux passages difficiles. Et ils menaient cette existence pendant plusieurs semaines consécutives pour essayer de faire descendre des lots d'Acajou vendus, et même payés d'avance, mais coupés en des endroits d'accès trop diffcile pour les modestes moyens d'exploitation dont ces coupeurs disposaient.

Arrivées à I'endroit où la " voie qui marche ") est suffisamment large et profonde, sinon toute l'année, du moins pendant une durée assez longue, les équipes qui accompagnent les billes rassemblent celles-ci et font des drômes ou radeaux. Une lance à boucle est fichée à l'avant, une autre à l'arrière des billes. On y passe des cordages ou des lianes qui amarrent les billes les unes aux autres. Il n'y a plus ensuite qu'à laisser filer tout doucement les drômes vers le point d'embarquement. Quelques hommes suffisent pour diriger le convoi. Il leur faut toutefois veiller, lorsqu'ils approchent de l'embouchure des fleuves (embouchure par laquelle les lagunes communiquent avec l'Océan), à ne pas se laisser entraîner par le courant à marée descendante. Ils doivent suivre de très près, et plusieurs kilomètres à l'avance, la terre du côté où ils doivent s'arrêter et diriger les drômes à la cordelle. Il est arrivé à plusieurs reprises, en effet, que des trains de bois, insuffsamment surveillés, ont été entraînés à la mer. Le repêchage est d'autant plus difficile que les amarres sont rompues au passage de la barre et que les billes, rejetées le long de la plage, dispersées peu à peu par les courants qui suivent la côte, ne quittent guère la zone où la barre exerce son action.

Le flottage des bois n'est donc pas, à la Côte d'Ivoire, sauf pour les chantiers privilégiés desservis par les lagunes ou de bonnes rivières, un moyen d'évacuation de tout repos. En outre, il n'est utilisable que pour les bois de faible densité ou de densité moyenne. Il ne permet pas l'exploitation des bois luurds qui ne flottent pas; et, s il permet celle des bois qui peuvent flotter après un certain temps de séchage, comme l'Iroko, le Dabéma et plusieurs autres parmi les plus beaux, ce n'est pas sans créer des complications. C'est une des raisons pour lesquelles l'exploitation de l'Iroko, bois de toute première qualité et d'écoulement facile à un prix voisin de celui de l'Acajou, n'a pas pris, jusqu'à présent, plus d'extension.

\section{Transport par chemins de fer.}

L'Administration du Chemin de fer de la Côte d'Ivoire met à la disposition des exploitants, lorsque ceux-ci en font la demande, des wagons pour le transport de leurs bois d'un point quelconque de la voie ferrée à Abidjan. Le chargement et le déchargement des billes, en grumes ou équarries, sont faits par les intéressés et à leurs risques et périls, l'Administration se bornant à faire remorguer les wagons. Ces opérations doivent être faites rapidement 


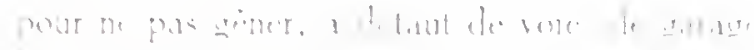

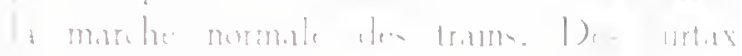

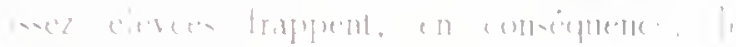

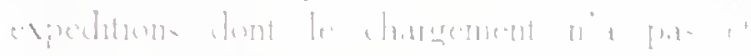

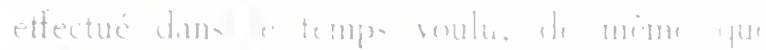

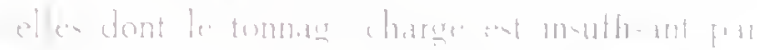
repport au nomber te warere emplose

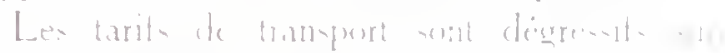

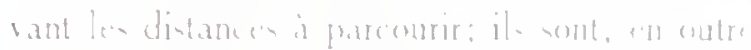

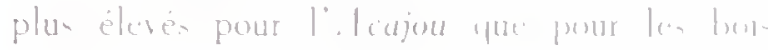

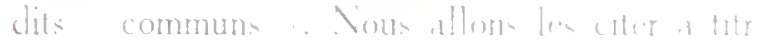
dindication :

1 Bois rentrant alans la | catequrie: teajou. Tiama et 1 rolen

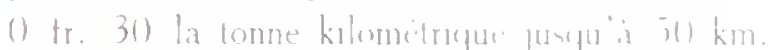
() Ir. 20 - - a delia il) $\mathrm{hm}$.

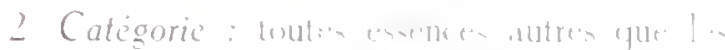

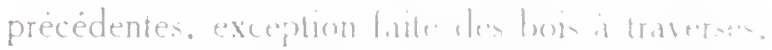

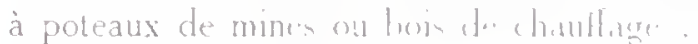

1) fr. 20) la tonne hilometriqus juspu"is ill $\mathrm{km}$. () fr. 1 i - - au deli le i) $\mathrm{km}$.

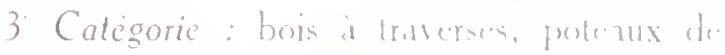
mines et bois de chaulluge :

1) fr. Ij la tonne kilometrugae jurqua a in $\mathrm{km}$. $11 \mathrm{fr} .118$ - - au lelir de $30 \mathrm{~km}$. Ces prix sentendent par eheresement d'at

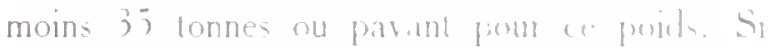
le chargement atteint ou dipare $17 \%$ tonme-

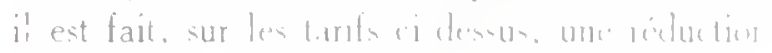
de 15

Tntrepoit des bois aus points d'cmbarquement.

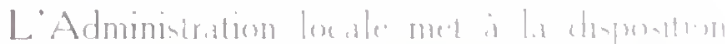
des exploitants, i Cirmol Banam at (irm!

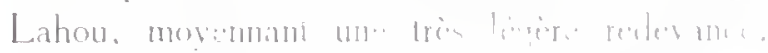

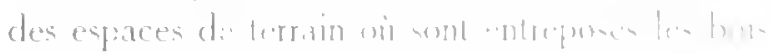
avant embarguement, \ Pawam, lo thm

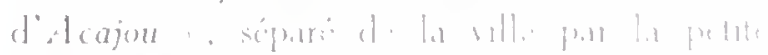

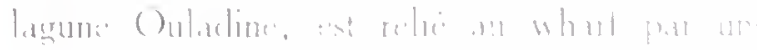

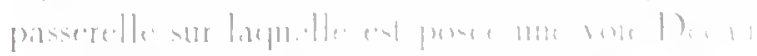

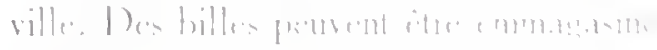

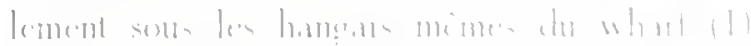

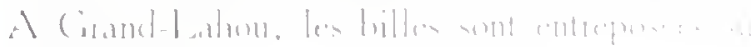

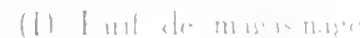
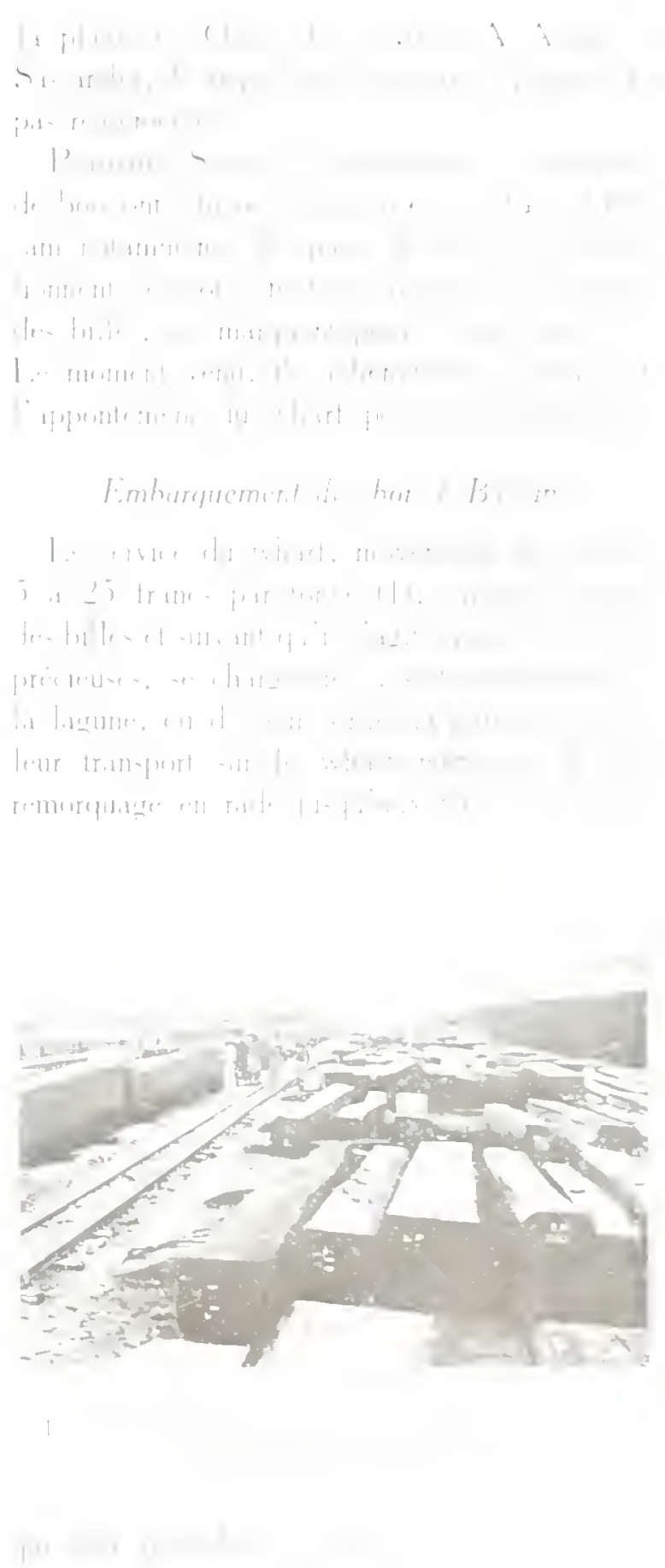
gement, lorsque plusieurs bateaux étaient en rade, étaient très lentes. Les expéditeurs, pour délicat fait par des équipes d'Appoloniens de l'endroit. Bien entendu, il était nécessaire que

\section{GRAND-BASSAM}

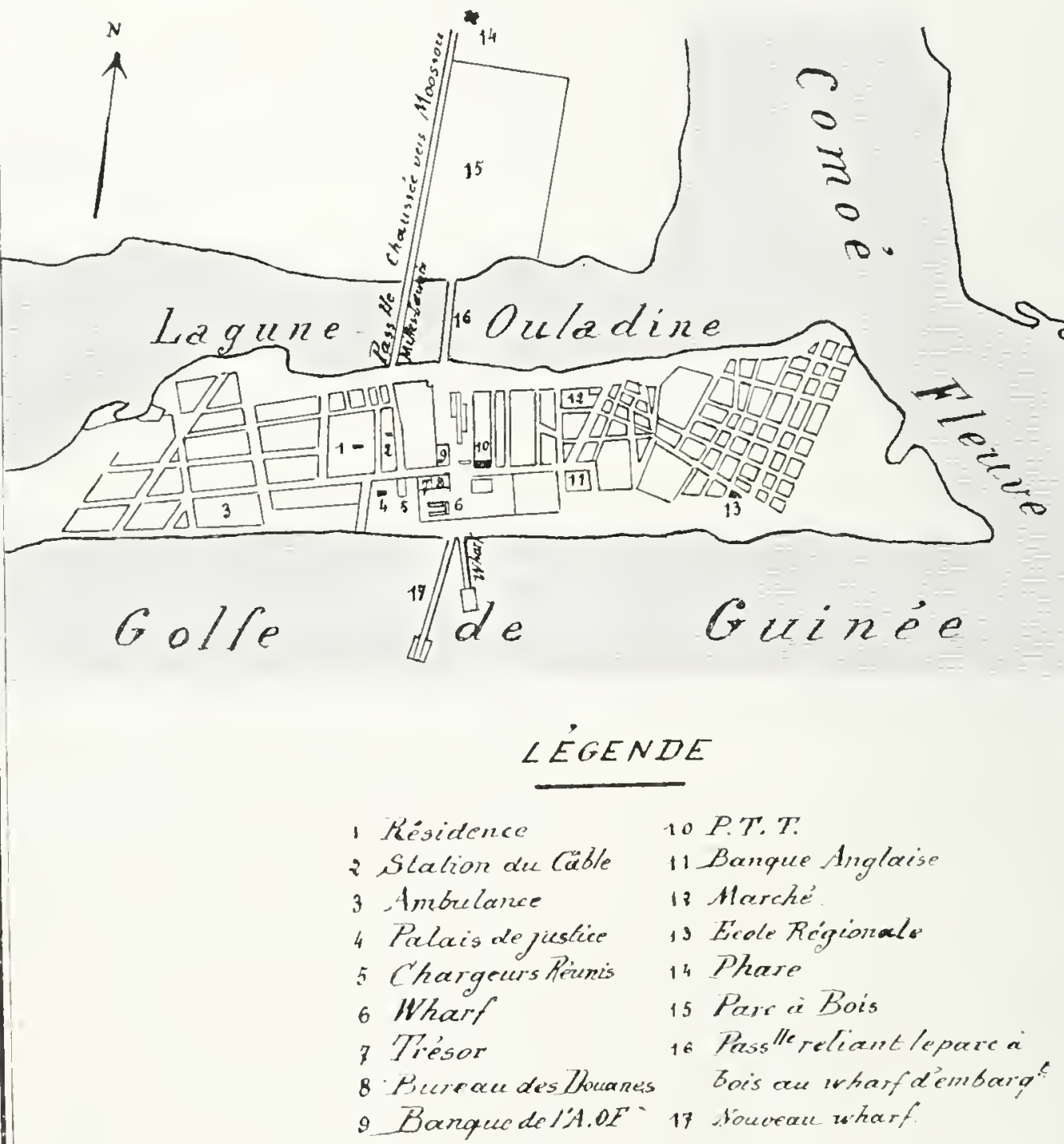

les accélérer et éviter le paiement de surestaries élevées, doublaient alors le trafic du wharf en faisant passer des billes à travers la barre, travail les billes à faire passer fussent conduites à l'avance sur la plage, à droite ou à gauche du wharf. L'achèvement de la construction d'un 


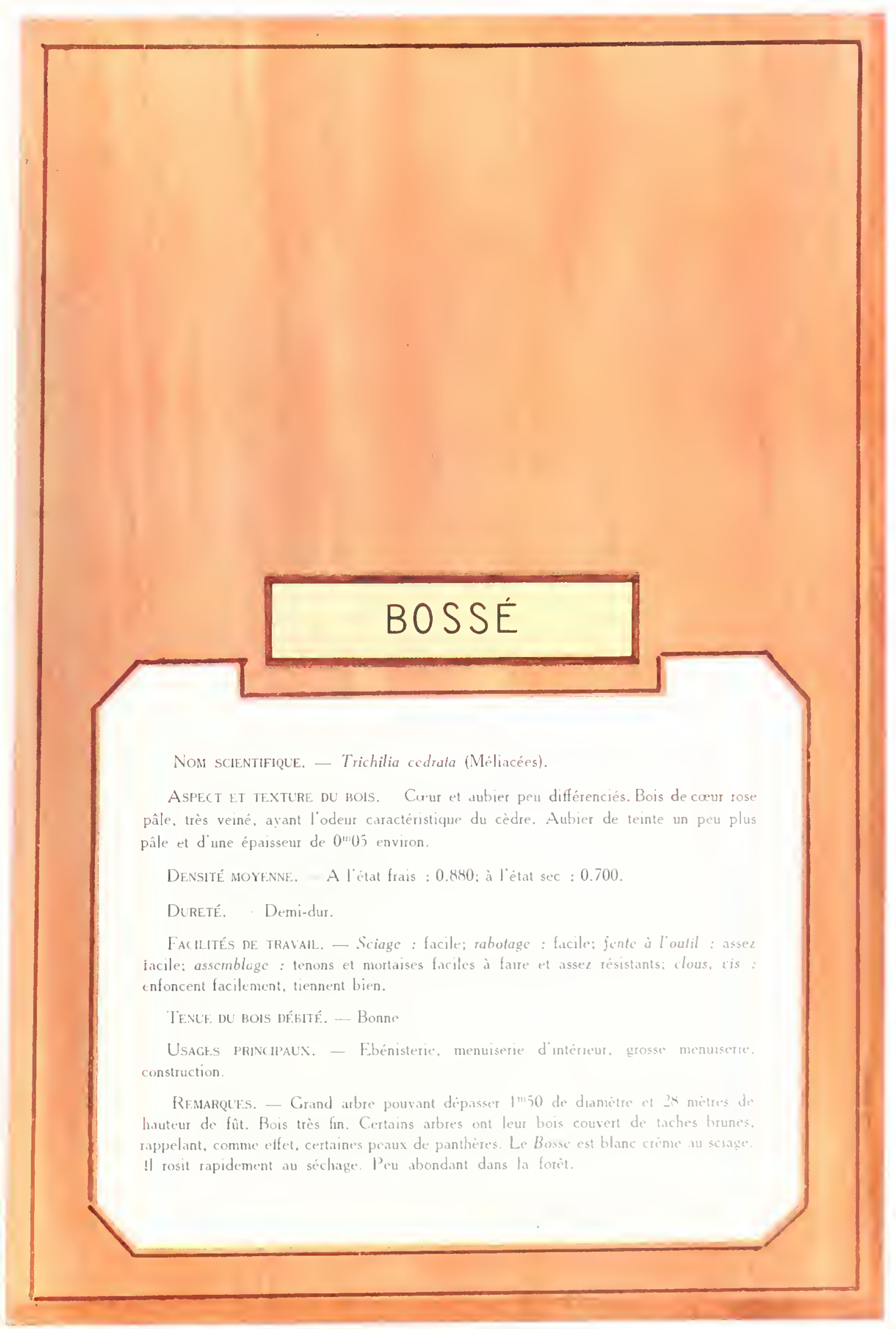


deuxième wharf, bien plus puissamment outillé que l'ancien, va sans doute permettre d'abandonner cette pratique (I).

Il n'est pas non plus impossible qu'on arrive à utiliser, par la suite, l'embouchure du Comoë pour le passage, soít de remorqueurs tirant des radeaux, soit de bateaux spéciaux transportant les billes de la lagune à la rade la barre, puis être remorquées ou conduites par des rameurs auprès des bateaux. Le chargement sє fait néanmoins assez rapidement, sauf lorsque ta barre est trop forte, et n'est pas, en somme. beaucoup plus coûteux qu’à Grand-Bassam.

A Sassandra, la barre est un peu moins violente que sur les autres points de la côte. Quoi qu'il en soit, il ne peut guère être question,

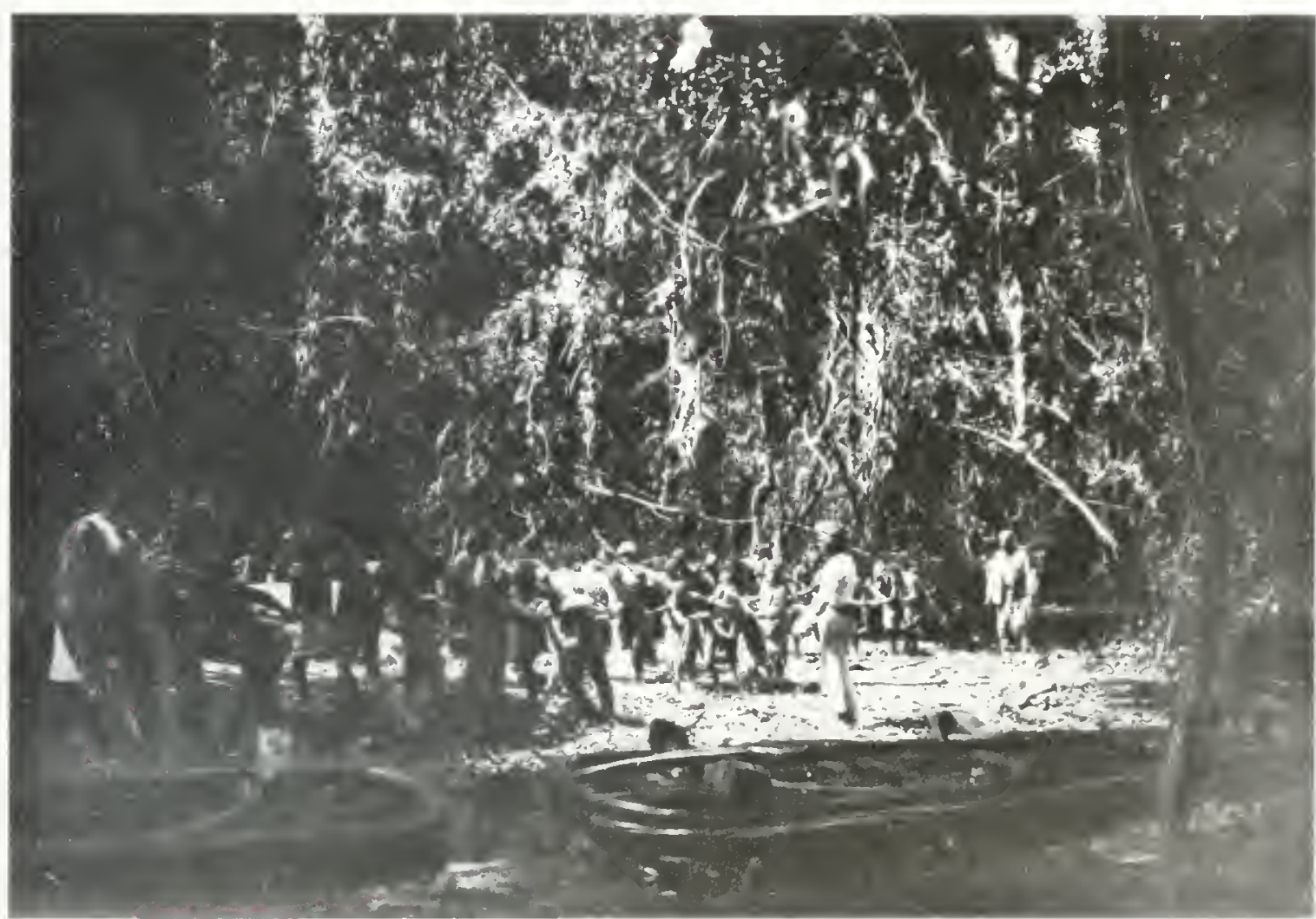

En foret. Tuage des billes sur rouleaur Cliche Mélayer. - Grand-Basam

foraine où sont ancrés les cargos de passage (2)

Embarquement à Assinic, Lahou et Sassandra.

A Assinie, à Lahou, à Sassandra où il n'y a pas de wharf, les expéditeurs n'ont pas le choix des moyens : les billes doivent passer

(1) On sait quiun troisième whart est prévu à Vridi. en face d'Abidjan.

(2) Nows ne parlerons pus ici des projets de port en eau calme, dont la réalisation ne sera peut-itre pas un fait accompli avant vingt ou trente années. Ce sera loaflaire d'une autre génération. (pas plus qu'à Lahou et à Assinie). d'y charger des bois ne flottant pas ou des bois sciés. avec les sculs moyens d'embarquement dont on dispose actuellentent. Cet inconvénient réduit en fait, momentanément. à la zone desservie par Bassam, l'exploitation des hois lourds et l'installation des scieries à grand débit.

Il existe cependant, pour las côtes inhospitalières du genre de celle qui nous occupe. un procédé qui, s'il était emolové et génèralis. serait de nature à améliorer considérablement 
les conditions de chargement des bois de la Côte d'Ivoire. C'est l'usage de la "touline ", long et fort câble de chanvre ou filin sans fin, monté sur un treuil à vapeur installé sur le navire (lequel est ancré au large) et relié à une poulie fixée à l'endroit de la plage où sont placées les billes à embarquer. Le treuil mis en mouvement, le câble tourne comme le ferait une courroie de transmission, mais assez lentement. II suffit alors d'amarrer de distance en distance les billes à ce câble; au fur et à mesure qu'elles arrivent auprès du navire, elles sont sorties de l'eau et hissées par les appareils de levage du bord.

Ce système présente, d'autre part, le grand avantage de charger des bois de densité légèrement supérieure à celle de l'eau, surtout si les billes lourdes sont réparties entre celles de densité plus légère, faisant office de flotteurs.

Une touline coûte évidemment assez cher. De plus, elle s'use, il faut la remplacer assez souvent. Les services à attendre de son fonctionne.. ment sont tels, dans les points dépourvus de tout autre outillage de chargement, qu'il semble pourtant y avoir un très gros intérêt à l'utiliser. Si les Compagnies de navigation qui fréquentent la côte ou, à leur défaut, les Sociétés ou particuliers ne veulent en faire les frais, l'Administration locale, après entente avec les exploitants, pourrait sans doute tenter l'entreprise et prévoir une taxe pour l'amortissement des frais d'achat. Nous sommes certains que l'adoption de ce procédé de chargement sur les divers points de la côte, et même au wharf de Bassam, amènerait immédiatement, par la plus grande rapidité des embarquements, une réduction sen. sible des tarils de fret appliqués aux bois en billes, tarifs qui pèsent lourdement sur les bois autres que ceux d'ébénisterie et leur permettent difficilement de concurrencer, sur les marchés français, les essences d'emploi similaire provenant du Nord ou de l'Europe centrale.

\section{Droits de sortie.}

Signalons ici que les bois sont assujettis, comme la plupart des autres produits de cueil- lette de la Colonie, à des droits de sortie perçus par le service des Douanes. Ces droits ne s'appliqueni toutefois qu'à l'Acajou et aux essences d'ébénisterie (en fait, on ne l'applique, en dehors de l'Acajou, qu'au Tiama et à l'Iroko). Depuis janvier 1921, ils ont été portés de 4 à 12 francs par mètre cube.

\section{Transports maritimes.}

Trois Compagnies françaises de navigation : Chargeurs Réunis, Fraissinet, Société Navale de l'Ouest, ont des cargos qui fréquentent assez régulièrement la Côte occidentale d'Afrique.

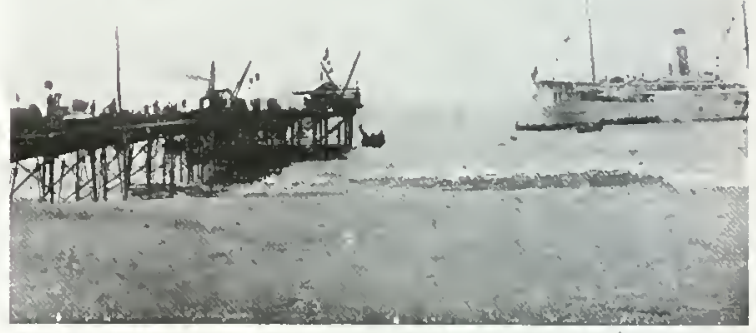

Le whart de Grand-Bassam. Cliche Agence economique A. O F.

On voit également, de temps à autre, un bateau de la Compagnie Fabre ou de la Compagnie Paquet, ou des bateaux de Compagnies diverses, affrétés spécialement pour des transports de bois. Concurremment avec les lignes françaises, touchent à Bassam et, le cas échéant, sur les autres points de la côte, des cargos anglais, allemands, américains, hollandais, etc.

Ces différentes Compagnies sont plus ou moins exigeantes, leurs bateaux soni plus ou moins bien outillés et aménagés pour le transport des bois. Nous ne ferons ici de critique ou de réclame au détriment ou en faveur d'aucune d'elles. Mais nous pouvons dire que, pendant la période qui a suivi la guerre, en 1919 et en 1920, les expéditions de bois ont été très 
sérieusement entravées par l'insuffisance marquée du nombre de bateaux, en même temps que par l'élévation considérable des tarifs de transport. La situation s'est sensiblement améliorée à cet égard et, depuis quelques mois, if est devenu possible d'expédier à bien meilleur compte et au moment où l'on peut désirer le faire. Cela tient un peu à la crise commerciale qui a raréfié les échanges dans les différentes parties du monde; cela tient surtout, pour la Côte d'Afrique, et la Côte d'Ivoire en particulier, à ce que les Compagnies qui desservent cette partie de notre domaine colonial ont construit ou acheté de nouveaux bateaux dont certains sont puissamment outillés pour le chargement des bois en grumes ou équarris.

Néanmoins, il est prudent, lorsqu'on a un assez fort chargement à faire, de s'entendre à l'avance avec les Compagnies pour retenir le fret nécessaire. Certaines Sociétés, qui expédient plusieurs milliers de tonnes à la fois, continuent du reste à affréter en France des cargos qu'elles envoient spécialement là-bas pour prendre leur stock. II n'est pas inutile de recommander aux non initiés qui seraient tentés de les imiter, de bien choisir le bateau à envoyer. Il en est venu, en effet, quelques-uns, à Bassam ou à Lahou, durant la guerre, et même après, qui n’étaient nullement équipés pour charger et transporter des bois en billes: les uns ont mis plusieurs mois à faire leur chargement; les autres. qui croyaient prendre 6.000 tonnes, sont repartis péniblement avec 2.000 ! Enfin, il est prudent de prévoir un assez long délai pour le chargement afin d'éviter le paiement de surestaries.

Les tarifs de transport, tombćs pour les bois de 400 francs au milieu de 1920, à 100 francs et même 90 francs la tonne (1) à la fin de 1921 , pourront sans doute être abaissés eneore par la suite (2), quoique le maintien des prix du charhon, celui des salaires du personnel. l'applica-

(1) Pour les chargements d'une certaine importance.

(2) Ces tarifs ont, en effet. continué à baisser et dès maintenant (Maj 1922) il est possithle d'obtenir du fret pour les bois à 50 ou 60 francs la tonne. (Note de l'éditeur.) tion de la journée de huit heures entrainent de lourdes charges pour les Compagnies. N"oublions pas, d’autre part, que la cherté des frets de ou pour la Colonie est due à la lenteur des cpćrations de chargement ou de déchargement et que tout moyen susceptible d'activer ces opérations aura une répercussion heureuse sur les tarifs.

\section{MaIN-D'EUUVE NÉCESSAIRE AUX EXPLOITATIONS FORESTIÈRES}

Les exploitations forestières, telles qu'elles sont organisées à la Colonie. nécessitent une

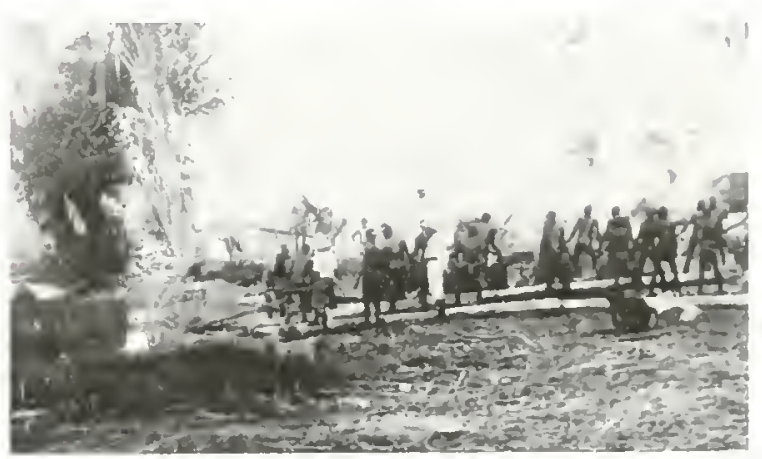

Moossou. - Tirage de l'eau d'une bille.

main-d'ourre considérable eu égard au tonnage de bois sorti des chantiers. On peut estimer que sur un chantier situé au bord d'un cours d'eau ou d'une roie quelconque d'évacuation. les billes sont tirées à main d'homme. en tenant compte de la sinuosité des chomins. sur une distance moyenne de 3 kilonètres si l'exploitant n'a pas de roie Decauville à sa disposition, et de 1 kilomètre s̈il en a. Les chantiers exploitćs sans matéricl Decauville sont presque aussi nombreux que les autres et on ne risque pas de se tromper beaucoup en déclarant qu- dans l'ensemble, les billes sont tirées à main d'homme sur les chantiers de 1 " zone (I) sur une distance morenne de 2.000 mètres. En 2 et 3 zones. la distance à faire parcourir par les

(1) C.est-à-dire en bordure des voies d"eracuation. 
équipes de tirage ne diffère pas beaucoup, car les voies Decauville posées, si elles sont indispensables, vont rarement jusqu'au fond des chantiers.

On peut donc établir, en principe, que tout chantier en activité, dont le rendement est de 800 à 1.200 tonnes de bois par an, doit disposer d'une équipe de 60 à 70 hommes, occupée en permanence au schlittage des billes obtenues; il faut, en plus, des hommes pour l'ouverture et l'entretien des chemins de tirage: il en faut pour l'abatage des arbres, le tronçonnage et l'équarrissage; il en f́aut pour charger et pousser les wagonnets Decauville, diriger les billes ou les drômes qu on fait flotter, etc.; au total : I20 à 125 hommes par chantier, chiffres auxquels il faut ajouter les cuisiniers ou cuisinières, les porteurs de vivres, etc. Les 50 à 60 chantiers qui étaient en pleine activité en 1920 occupaient donc au total près de 8.000 bûcherons ou manœuuvres. Et c'est là le nombre minimum d'hommes qu'on verra de nouveau sur les chantiers dès que l'écoulement des Acajous sera redevenu normal.

Où et comment se recrute cette main-d'œuvre et comment la conserve-t-on sur les chantiers? On sait, en effer, que le travail demandé à ces ouvriers est d'autant plus pénible que, assez fréquemment, ils doivent haler les billes dans des terrains marécageux, dans ce qu'on appelle, à la Côte d'lvoire, le " poto-poto ", où ils enfoncent parfois jusqu'à mi-jambe. La Colonie est, d'autre part, relativement peu peuplée et les populations de la région qui constitue la zone exploitable de la forêt, à l'exception des Abeys (qui fournissent d'excellents bûcherons pour l'abatage et l'équarrissage des bois) sont plutôt réfractaires au travail des chantiers; elles ne s'engagent pas volontiers et ne fourmissent qu'un rendement assez médiocre. Elles gagnent d'ailleurs davantage, lorsqu'elles veulent exercer leur activité, à faire quelques cultures de bananes, manioc, ignames, dont elles vendent les produits aux exploitants forestiers du voisinage, et à récolter quelques régimes des innom- brables palmiers à huile qu'elles détiennent. Les services publics, l'entretien des routes et surtout le portage, absorbent, par ailleurs, la majeure partie de la main-d'cuvre gui n'est pas employée aux cultures.

Les exploitants forestiers réussiżent néanmoins à réunir sans trop de difficultés le nombre d'hommes qui leur est nécessaire, soit qu'ils engagent sur place des volontaires venant un peu des quatre coins du pays, mais surtout du Nord (I), soit qu'ils aillent faire du recruiement dans les cercles du centre ou du nord de la Colonie, et paszent, avec l'autorisation de l'Administration, des contrats de louage avec les indigènes de ces régions. Les engagements, dans ce cas, se font habituellement pour six mois sur la base suivante :

1 fr. 75 environ par journée de travail; ration en naiure, comprenant 600 à 700 grammes de riz, ou quantité correspondante de bananes, ignames, manioc, etc.; huile et poisson une ou deux fois par semaine, sel en quantité suffisante. Le dimanche, il est d'usage de distribuer du tabac. On engage en même temps une cuisinière, par vingt hommes, payée I franc par jour. Les chefs d'équipe sont choisis par les hommes eux-mêmes; ils touchent de 2 fr. 50 à 3 fr. 50 par jour.

L'employeur est tenu de loger ses ouvriers; des huties sont construites à cet effet sur les chantiers. Il leur prête, en outre, des couvertures pour la nuit. En les traitant bien, en n'exigeant pas d'eux un travail au-dessus de leurs forces, on obtient de ces hommes un assez bon rendement. Ils reviennent volontiers sur les chantiers où ils ont été bien traités et bien nourris. Ils désertent, par contre, sans souci de leur engagement, ceux où l'on exige d'eux un travail trop dur, où la ration est insuffisante. L'employeur a donc tout intérêt à se lcs attacher par de bons procédés.

D’une façon générale, les ouvriers indigènes préfèrent toucher un salaire moindre et être

(1) Bambaras à qui le dur travail des chantiers ne répugne pas. 


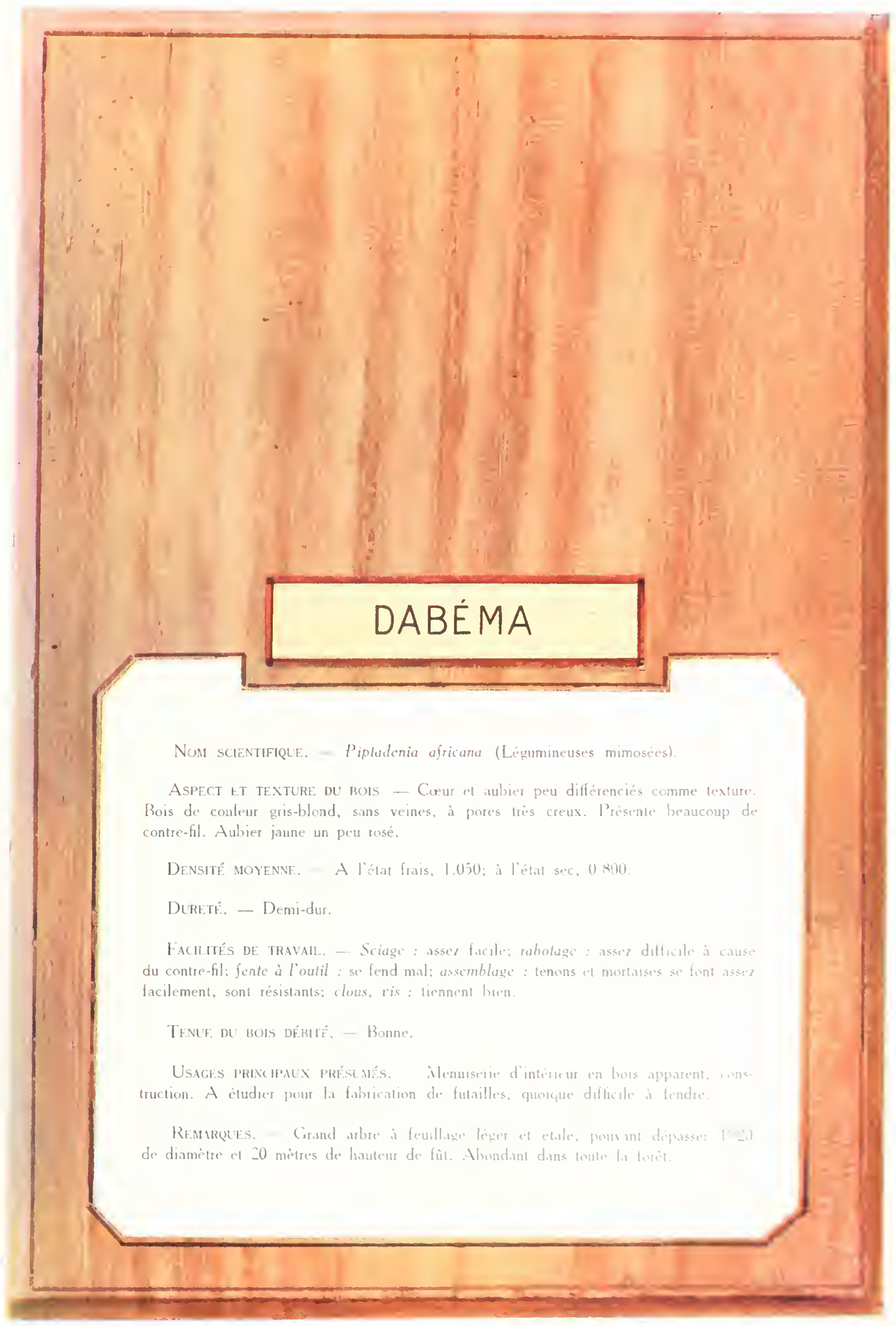



abondamment nourris. L'ancien système, qui consistait à remplacer les vivres par une indemnité en espèces, est à condamner. Les hommes, qu "ils aient joué et perdu leur argeni, ou qu'ils aient des difficultés à se procurer les aliments qui leur sont nécessaires, mangent insuffisamment ou mangent des aliments mal préparés. Ils ne peuvent fournir longtemps l'effort qui leur est demandé.

L'Administration veille à l'exécution des contrats d'engagement, dont les stipulations doivent être en concordance avec les principes généraux énoncés dans la réglementation forestière, notamment en ce qui concerne la durée de la journée de travail, le nombre de journées de travail à exécuter par semaine ou par mois, les conditions de salaires, de vivres, de voyage, de couchage, etc.

Le prix de revient moyen de la journée de travail d'un ouvrier indigène est très variable suivani le plus ou moins de cherté des vivres nécessaires à son alimentation. Avant 1914, en année normale, on cstimait qu' une journée de manœuvre revenait au total à $\mid$ franc ou I fr. 25 (1), dont 0 fr. 25 d'indemnité de ration. Aujourd'hui, elle coûte beaucoup plus; les salaires ont doublé d'une façon générale pour le personnel noir et la ration en nature qui est accordée aux travailleurs revient à beaucoup plus de 25 centimes. En 1920. lorsque les exploitants achetaient 6.000 francs la tonne de riz qu ils destinaient à leur hommes et que les autres produits étaient payés à l'avenant, la ration de vivres pouvait être évaluée entre $3 \mathrm{fr} .50$ et 4 francs! La journée de travail d'un manœuvre revenait donc à plus de 6 francs, en tenant compte des jours de repos, de voyage, de maladie, des gratifications, etc. Depuis, cc prix de revient s'est sensiblement abaissé. les denrées alimentaires récoltées sur place étant plus abondantes. Il doit varier, suivant les époques de l'année, entre 2 fr. 50 et 3 fr. 50 .

Les bûcherons Abey's préfèrent souvent, à la ration en naturc, une indemnitć en cspèces: lcur

(1) Le Barbier : La Côle d'lione. page 149. salaire total atteint ainsi de 3 francs à $3 \mathrm{fr}$. 50) par jour.

Les exploitations se développant, le recrutement de la main-d'euvre restera-t-il possible? Il est bien certain que, pendant quelques années, les travaux de chemins de fer et de routes. dont l'exécution est projetée, vont nécessiter un grand nombre de travailleurs. Mais il est à prévoir que beaucoup de ceux-ci viendront $\mathrm{s}^{\circ} \mathrm{em}$ baucher d'eux-mêmes ou seront envovés des régions du Soudan et de la Haute-Volta. Ces Colonies sont, en effet, intéressées au premier chef, au prolongement rapide de la voie ferrée

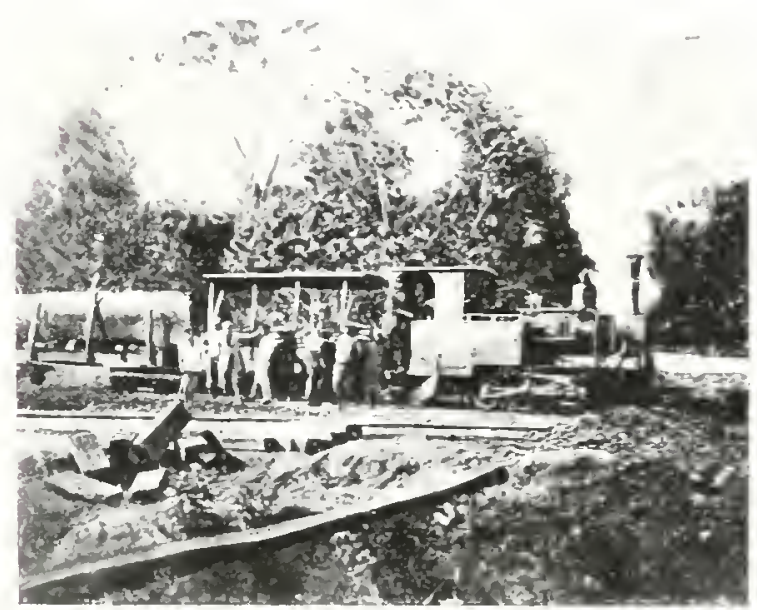

Tran de billes.

Cliche Metayer Grand-Bussam

qui monte vers le Nord: leur Administration nc pourra donc que faciliter cet exode de travailleurs. Peu à peu, ces mêmes travailleurs descendront jusquà la côte et iront librement śengager sur les chantiers forestiers. comme ils ront déjà en Gold-Coast s'engager pour les travaux miniers ou agricoles.

Enfin. l'emploi de voies Decaurille, d'autres moyens perfectionnés d'évacuation des bois se généralisera de plus en plus, et il n'est pas téméraire d'avancer qu'avec la même main-d'ourre dont ils disposent actuellement. les exploitants sortiront. dans quelques années, de leurs chantiers. un tonnage de hois double ou triple de celui qu'ils sortent actuellement. 


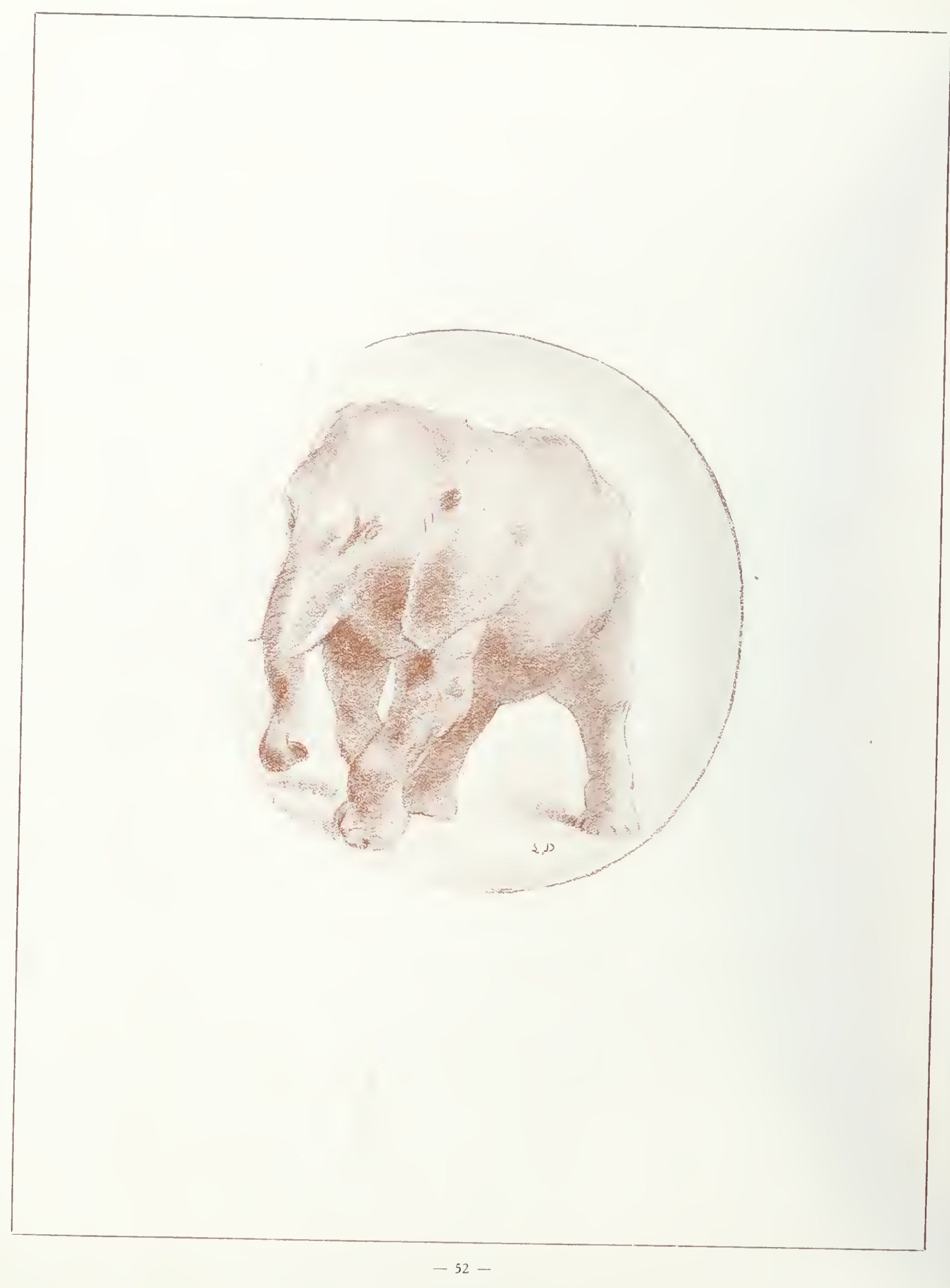




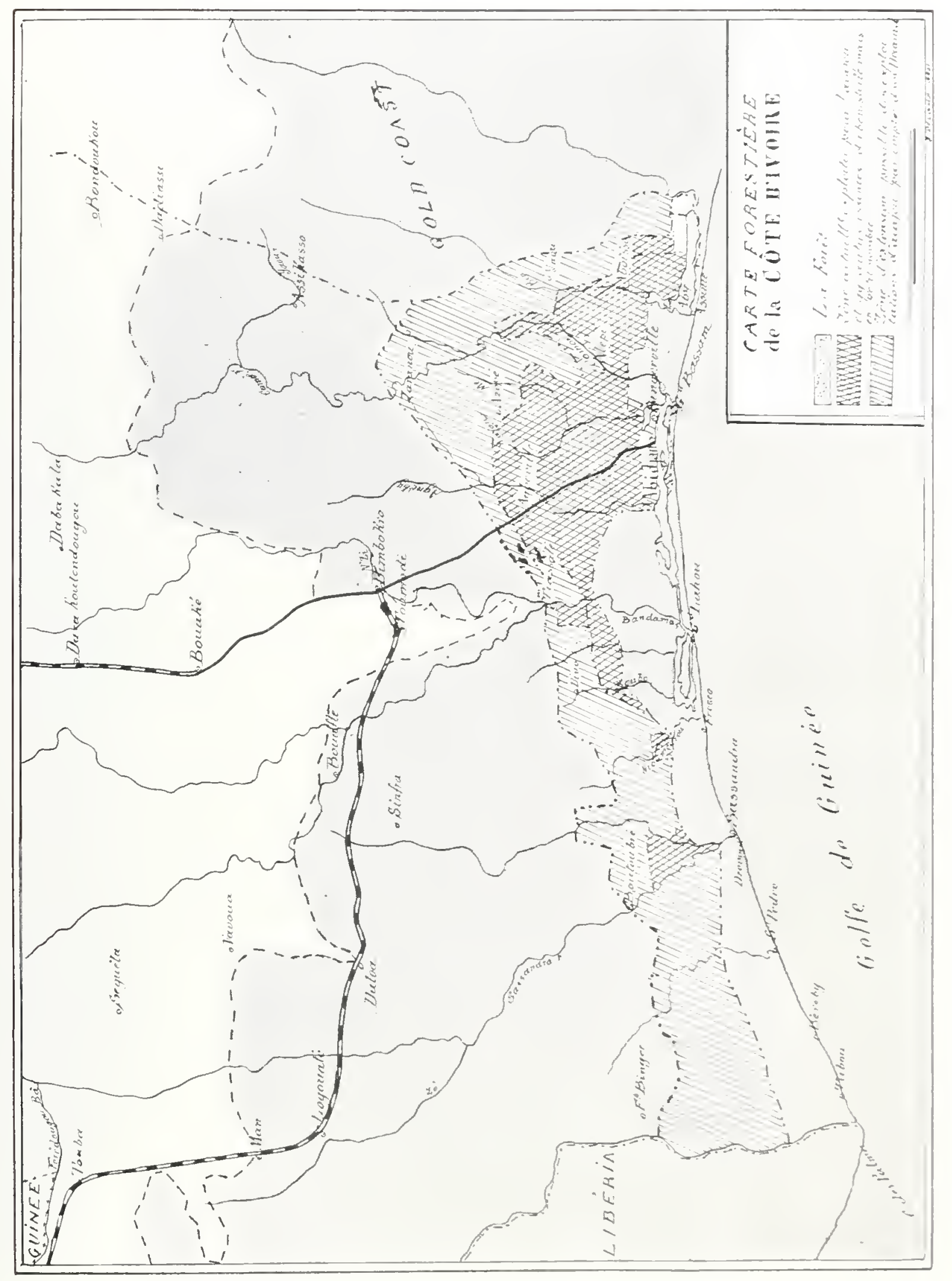





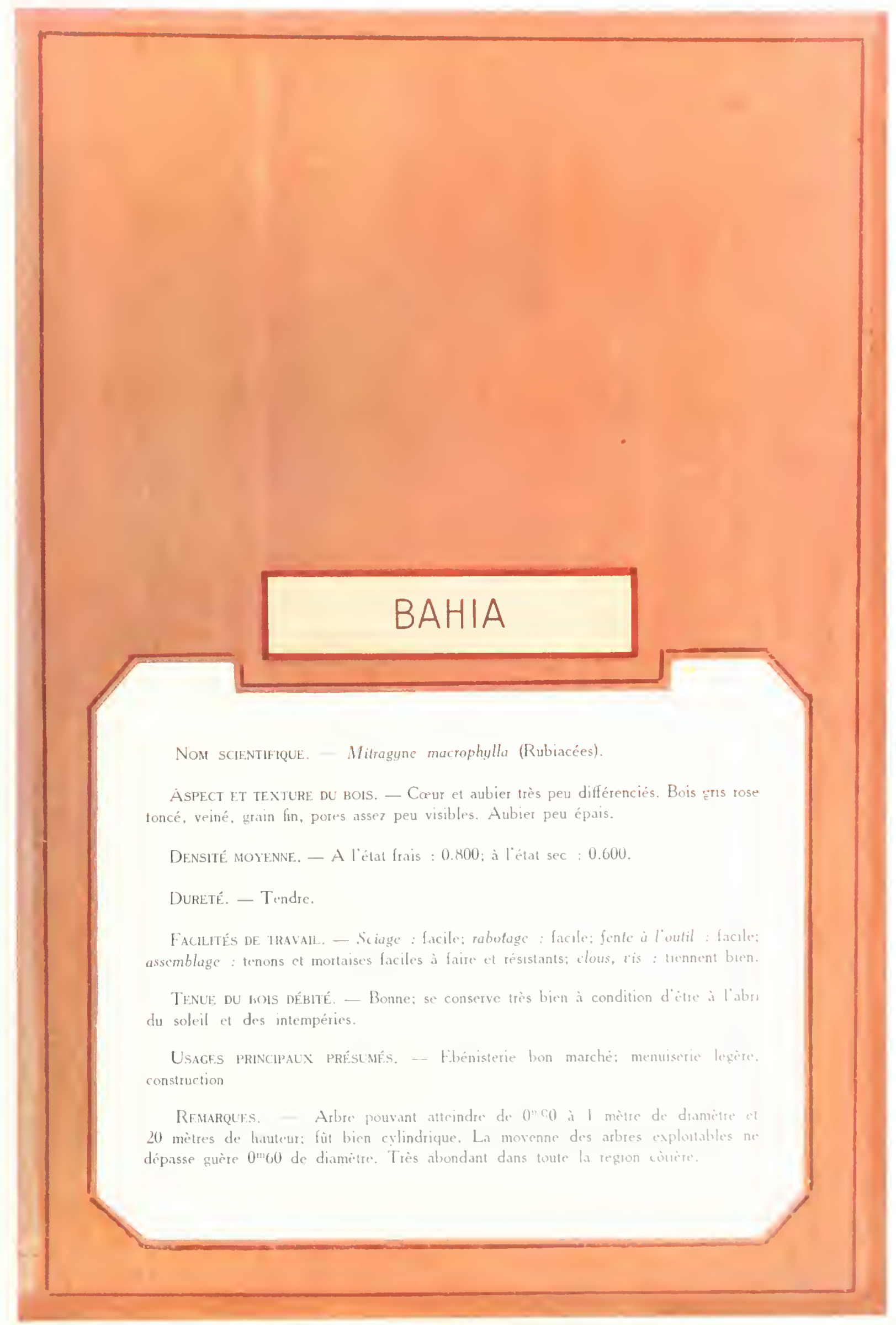





\title{
TITRE II
}

\section{Les exploitations actuelles Bois précieux et bois communs}

\author{
SOMINAIRE
}

Les cuploitations actuclles. - Capil.d nécessaire à une cxploitation - Prix de revient des hois exploités el prix de vente. - Production el déhouchés. - Qualité des Acajous ie la Côte d'troire. - Les scrieris locales; écoulement de leur production. 


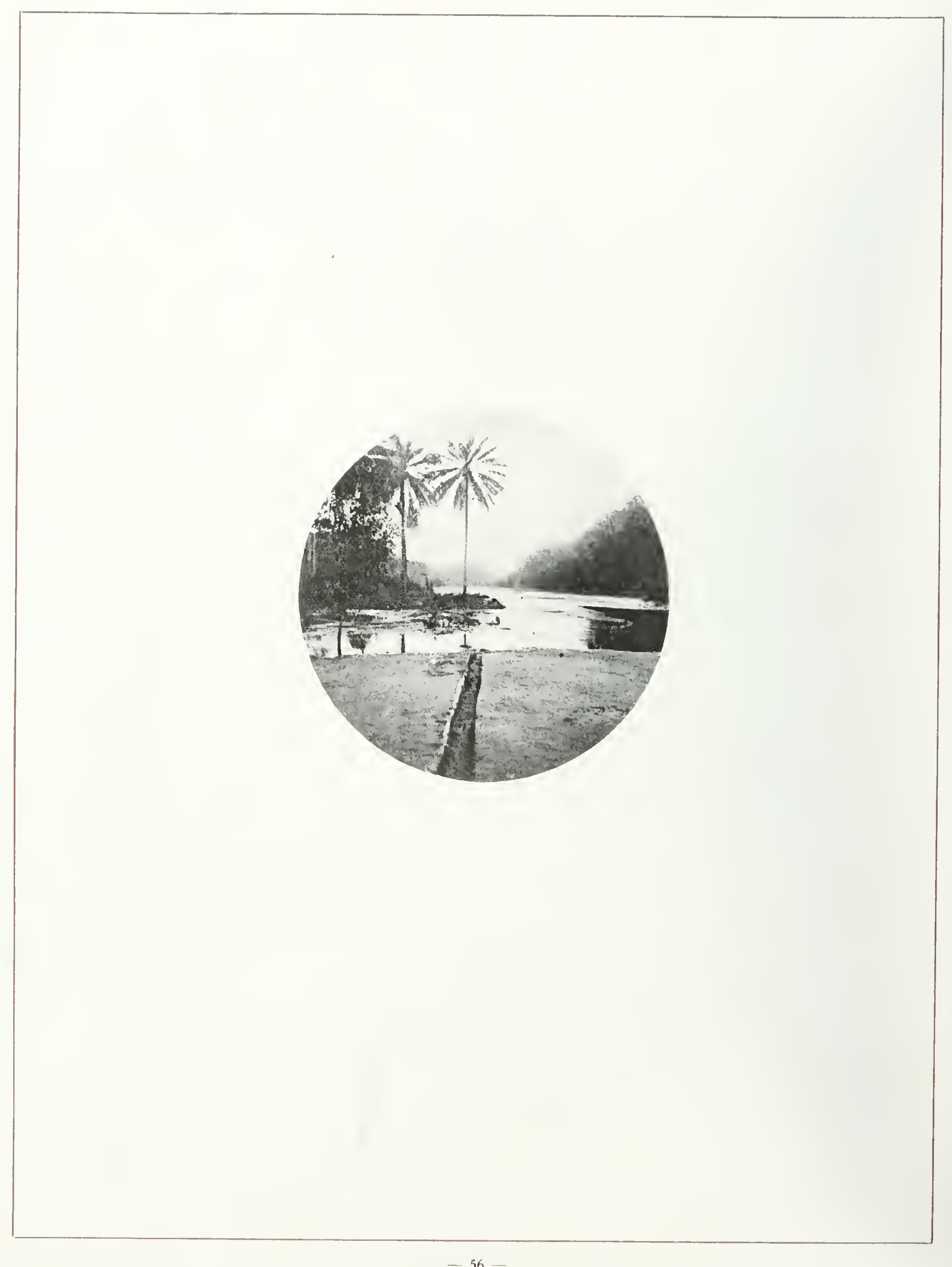


TITRE II

\section{Les Exploitations actuelles. - Bois précieux et Bois communs}

\section{Les Exploitations actuelles}

Nous avons vu, précédemment, que la superficie actuellement concédée de la forêt de la Côte d'lvoire atteignait environ 650.000 hectares. En déduisant la concession de la Compagnie Forestière de l'Afrique française (60.000 hectares), c'est près de 240 chantiers, de chacun 2.500 hectares, qui sont ainsi détenus par une quarantaine de Sociétés ou particuliers se livrant à l'exploitation des bois.

Tous ces chantiers ne sont pas exploités et il en est bien près de la moitié où la hache n'a pas encore pénétré. Au plus fort de l'année 1920, qui fut si favorable à certains exploitants, et eût dû l'être à tous, sans exception, on ne comptait guère qu'une soixantaine de chantiers en activité. Le nombre a certainement diminué depuis. les coupeurs, plus ou moins gênés par la crise commerciale qui a rendu très difficile, même à bas prix, l'écoulement des bois, ayant peu à peu fermé un certain nombre de leurs chantiers et réduit, au minimum, le personnel laissé sur les autres. Le nombre de chantiers, c'est-à-dire la superficie concédée, n’est donc pas en rapport avec le nombre et l'importance des exploitations. Pourquoi? On va s'en rendre compte aisément. A la faveur de la hausse des bois. fin 1919 et $\mathrm{I}^{\text {er }}$ semestre 1920, période al cours de laquelle les $A$ cajous en billcs équarries pas- sèrent, progressivement, de 180 à 1.100 francs la tonne, quai départ Colonie, il y eut comme

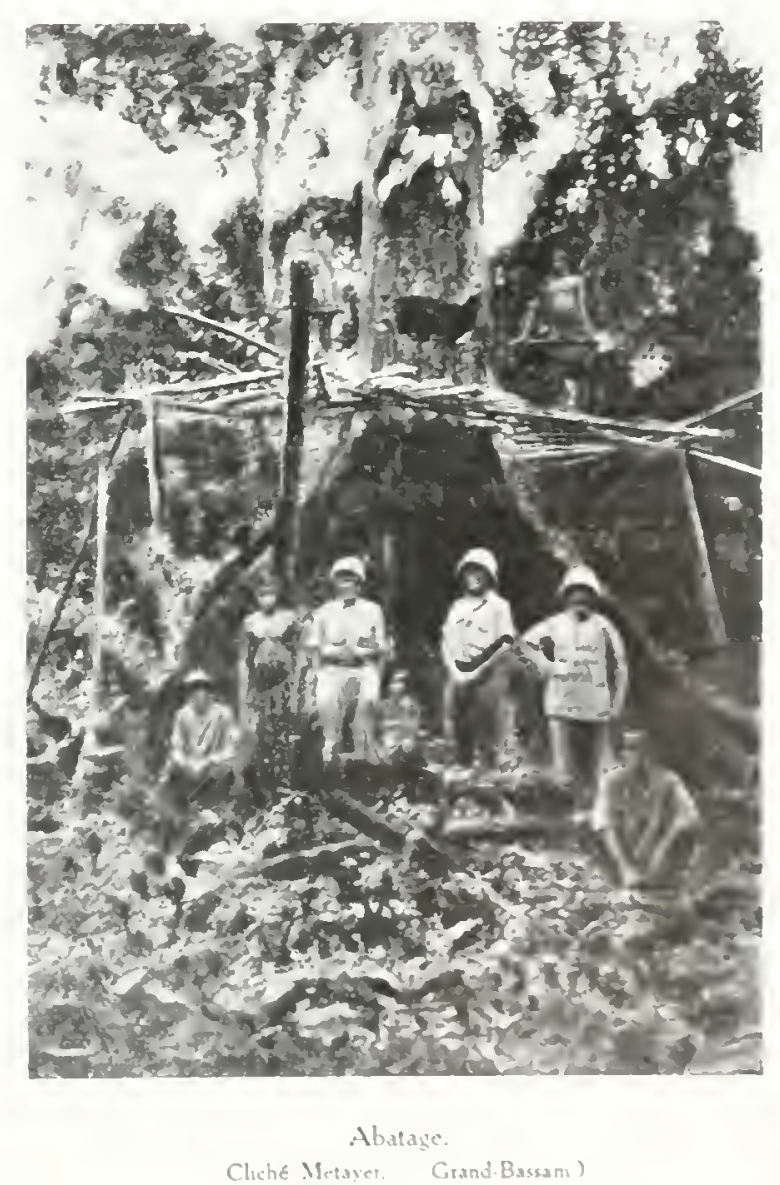

un rush sur la forct. Tout coupeur qui avait un chantier chercha à en avoir trois ou quatre; les 
Sociétés qui possédaient dix chantiers, en voulurent trente, quarante. II fallait se créer des réserves pour l'avenir, car l'appìt du gain n'allait pas tarder à faire surgir de nouveaux exploitants, désireux, eux aussi, de faire fortune en quelques mois, et il ne fallait pas que les nouveaux venus vinssent gêner les anciens, se mettre à côté d'eux ou, immédiatement en arrière. On envisageait, en même temps, et à ce point de vue on avait raison, que les bénéfices réalisés allaient permettre l'achat de matériel perfectionné, la construction de chemins de fer Decauville, et qu il était nécessaire, pour échelonner l'amortissement de ce matériel et des frais d'installation, d'avoir la certitude d'exploiter dans une même zone pendant un certain nombre d'années. Enfin, on ne prévoyait pas que cette période, sans précédent, de gains considérables et faciles (on achetait presque indifféremment les bois de bonne ou médiocre qualité) serait de si courte durée et, surtout, serait suivie immédiatement d'une période de mévente presque absolue.

Les exploitants ont donc accru, un peu hâtivement, leurs frais généraux en accaparant des chantiers qu'ils ne peuvent momentanément exploiter. Et malgré les déboires qu'ils viennent d'éprouver, ils font tout ce qu'ils peuvent pour garder ces chantiers, espérant toujours en des temps meilleurs.

\section{Mćthodes d'exploitation. - Défauts et avantages.}

Si l'on fait exception de quelques coins de forêt exploités plus généralement pour l'alimentation des scieries locales, on peut dire que tous les chantiers exploités ne le sont en fait que pour l'A cajou.

Les quelques autres essences qui sont abattues par les exploitants, Tiama, Iroko, Badi, Niangon, Makoré, Bossé, ne sont coupées, en réalité, que si elles se trouvent à proximité d'un chemin de tirage, d'une voie Decauville ou d'une rivière servant à l'évacuation des billes. On ne les recherche pas. Leur valeur intrinsèque est certainement un peu moindre que celle de l'Acajou; certaines, comme l'Iroko ou le Makoré, flottent d'autre pari, difficilement à l'état vert: mais l'indifférence des coupeurs à leur égard vient surtout de ce qu'elles sont peu ou pas connues sur les marchés extérieurs et que leur écoulement est, quelquefois, difficile, alors que celui de l'Acajou, sauf en temps de crise comme celle que nous venons de traverser, est régulièrement assuré. On ne s'étonnera pas, par suite, que le tonnage réuni pour l'ensemble de ces essences, atteigne à peine le dixième de celui de l'A cajou.

Or, si nous nous en rapportcns à la documentation publiée par la mission Bertin, la proportion d'Acajous exploitables entre approximativement pour 1 o dans la composition de la forêt (l). A notre avis, le pourcentage réel de ces arbres est plutôt inférieur, et l'on peut tenir pour de très bons chantiers ceux qui ont $\mathrm{I} \%$ de leurs peuplements en $A$ cajous exploitables. Il est admis, en effet, qu'un hectare de forêt primaire, à la Côte d'lvoire, peut fournir facilement 300 mètres cubes de bois en grumes. Avec une proportion de $1 \%$ en $A$ cajous exploitables, on pourrait sortir une moyenne de 3 mètres cubes par hectare, soit 7.500 mètres cubes d'un chantier de 2.500 hectares. Or, ce chiffre n'est atteint qu'exceptionnellement, la moyenne des chantiers donnant de 1.500 à 2.500 tonnes d'A cajou, soit de 2.200 à 3.500 mètres cubes (2).

On voit, par cet aperçu, que l'exploitation de l'Acajou, si elle ne devait tendre à appauvrir progressivement la forêt au point de vue de la qualité des peuplements, ne lui enlèverait réellement qu'une bien faible partie du cube de bois qu'elle renferme. Et encore, dans la plupart des cas, les coupeurs ne se donnent même pas la peine de prospecter méthodiquement tout leur

(1) Tandis que les seules autres essences que nous indiquons doivent compter au total pour $5 \%$ environ.

(2) Il est vrai qu'il n'est coupé que des arbres ayant au moins $0 \mathrm{~m}$. 80 de diamètre au sommet de l'empattement, alors que l'ensemble esl coupé à partir d'un minimum de $0 \mathrm{~m} .30$. 


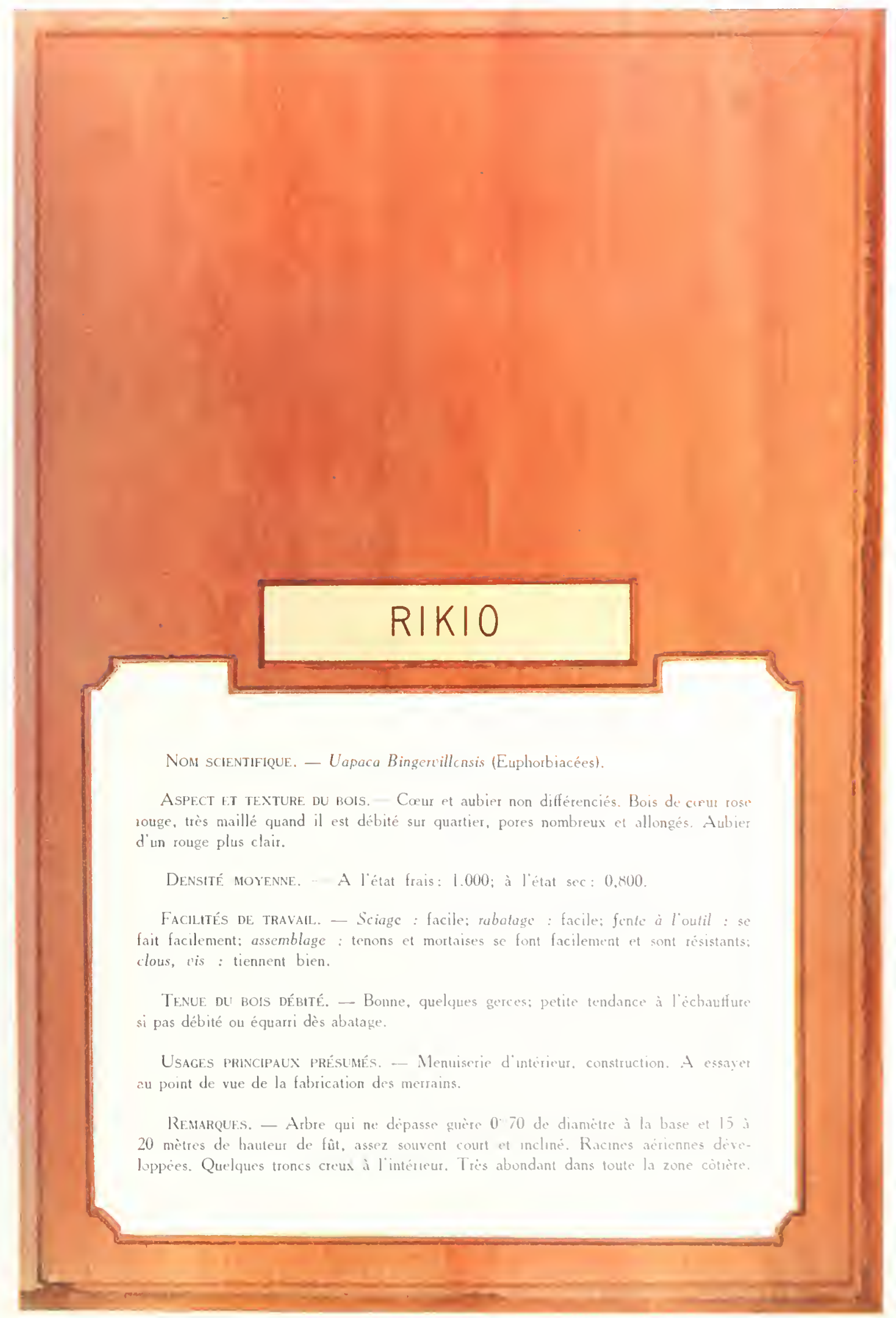


chantier. Ils coupent ce qui leur parait le plus facile à évacuer, puis abandonnent le chantier pour en entamer un autre. C'est ce qui explique que les zones de forêt qui avaient déjà été concédées et exploitées autrefois, sont sollicitées par de nouveaux coupeurs qui y trouvent quelquefois autant d'Acajous que dans les zones restées vierges.

Les exploitants sont donc à la recherche de bénéfices immédiats et faciles. Ils ne se soucient pas, ou ne se soucient que trop insuffisamment de l'avenir, ne cherchent pas dans l'organisation de l'écoulement des essences autres que l'Aca-

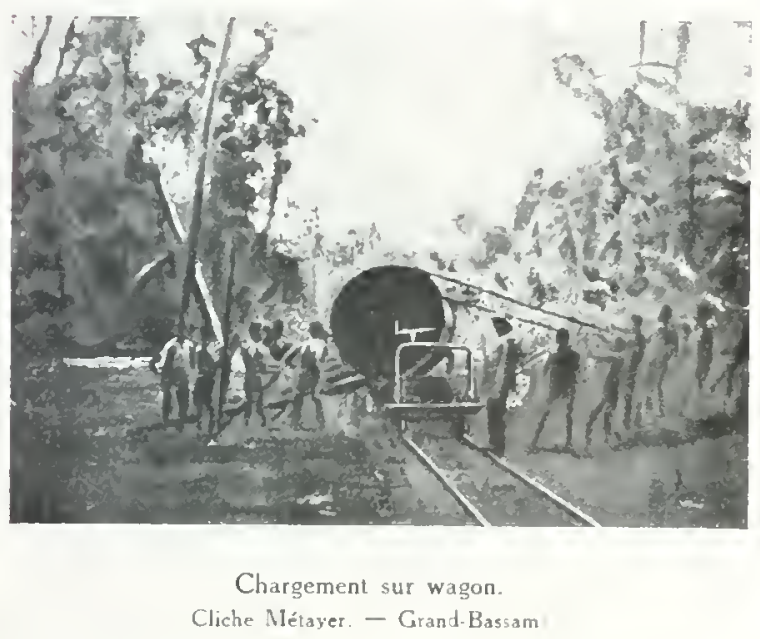

jou, une amélioration de leurs méthodes, un développement utile de leurs exploitations. Pour cueillir $1 \%$ ( $1 / 2 \%$ en réalité) des bois qui sont dans leurs concessions, ils doivent faire la même prospection, les mêmes chemins de tirage, les mêmes voies Decauville qu'ils feraient s"ils arrivaient à couper $5,10,20,30$ ou $40 \%$ du cube total. Résultat : leurs frais généraux sont relativement très élevés, le prix de revient des bois obtenus également. Quand les cours de l'A cajou sont avantageux, ils font néanmoins des bénéfices; en temps normal, les bénéfices se réduisent à bien peu de chose et, en temps de crise, les coupeurs travaillent à perte!

$C^{\prime}$ est que bien peu d'entre eux ont été préparés pour un tel métier: il en est de très intelligents, de très actifs, ınais, à de rares exceptions près, tous débutent avec des moyens excessivement modestes, tous emploient leurs capacités professionnelles à la recherche d'un résultat immédiat. Ils écrèment la forêt, réalisent et s'en voni. Il ne reste guère d'autres traces de leur passage ailleurs que les clairières dues à leurs déprédations. Ils ont versé quelques milliers de francs de redevances et de taxes diverses au Trésor de la Colonie et s'estiment quittes envers l'Administration de celle-ci, quand ils ne l'accusent pas d'être cause de leur échec s"ils n'ont pas réussi.

Devrait-on, en ce cas, limiter les autorisations d'exploiter, imposer des conditions très dures de mise en valeur des chantiers? A notre avis, il est préférable de continuer, au contraire, à se montrer très large dans leur atiribution et laisser évoluer librement la question exploitation, plutôt que de l'emprisonner dans une réglementation trop rigoureuse. On en est encore, à la Côte d'lvoire, au premier stade de l'exploitation forestière. 11 faut permettre aux premiers venus de faire fortune, s'ils le peuvent, pour que d'autres, tentés par les gains réalisés, viennent à leur tour monter des exploitations nouvelles. Peu à peu l'étude des bois se complète, la vente d'essences, encore inconnues hier, sorganise : des groupements, des Sociétés à gros capitaux se forment : c'est le deuxième stade. Nous sommes, à la Côte d'lvoire, peut-être plus près de ce deuxième stade que nous ne le pensons.

Nous avons, du reste, fait remarquer qu'il! avait parmi les exploitants actuels des exceptions au tableau que nous avons brossé. Il en est qui ont monté des scieries à la Colonie: d'autres qui se sont engagés dans la roie de plantations intéressantes. A eux seuls ils justifient la sollicitude de l'Administration à !'égard de la corporation. Noublions pas, enfin. que les coupeurs d'Acajou distribuent. en salaires et achats de vivres, une moyenne de huit à dix millions de francs par an aux populations indigènes. et qu "ils versent, en redevances et taxes diverses, huit cent mille francs à un million au Budget de la Colonie. Nous ne prétendons pas que tout cela 
constitue un ensemble merveilleux, mais ce sont là, néanmoins, des résultats non négligeables pour une première étape de l'exploiiation forestière. A côté des destructions, il y a un commencement de reconstruction et, surtout, d'organisation. C'est ce qu'il s'agit maintenant de développer.

\section{CAPITAL NÉCEssaire}

\section{A UNE EXPLOITATION D’ACAJOU}

Sauf quelques chantiers privilégiés situés au bord des voies navigables et dont la production peut être évacuée en permanence, réalisée au fur et à mesure qu'elle arrive au port d'embarquement, il faut, pour organiser une exploitation d'Acajou avec des chances de succès, un capital assez important.

Prenons, pour baser nos estimations, deux chantiers moyennement situés, l'un en deuxième z.one (1) du chemin de fer ou d'un cours d'eau à débit assez grand et assez régulier, l'autre en première zone d'une rivière à débit réduit et très irrégulier.

Pour le premier, il faudra six à huit kilomètres de voie Decauville, revenant, posée le plus sommairement possible, à 22.000 francs au moins le kilomètre, soit 130 à 170.000 francs; des wagonnets, de l'outillage, haches, passepartout, crics, etc., atteignant vite une vingtaine de mille francs; il $y$ a les redevances à payer à la Colonie, la prospeciion à effectuer, la main-d'œuvre à recruter, les chemins de tirage à ouvrir; enfin, il faut bien compter avoir à travailler trois à quatre mois, mettons six mols pour prévoir largement, avant de pouvoir réaliser les premiers lots de bois évacués. 130 hommes coûtant l'un dans l'autre, salaire et nourriture, 3 fr. 50 par jour, cela fait 430 francs par jour, 12.000 francs par mois, 72.000 francs pendant

(1) La première zone est constituée par les chantiers situés en bordure de ces voies d'évacuation; la deuxième zone, par les chantiers situés à 5 kilomètres de ces mêrnes voies. six mois. Si nous récapitulons, nous ne sommes pas loin de 250.000 francs.

Pour le second, il nous faudra un peu moins car nous ne compterons que trois kilomètres de voie Decauville (on pourrait même s'en passer complètement, mais, comme nous l'avons vu plus haut, chapitre "Moyens d'évacuation des bois "), ce matériel est indispensable si l'on veut exploiter économiquement un chantier). Par contre, les bois coupés ne pouvant être évacués qu'en période de crue, ce n'est plus six mois, mais une année qu'il faut compter entre le mo-

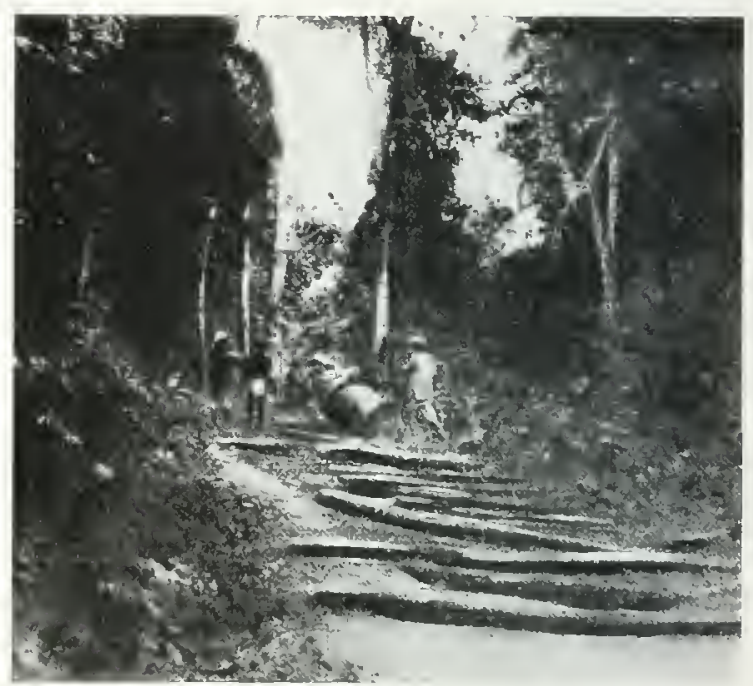

Chemin de rouleaux pour le tirage Cliche Métayer - Grand-Bassam

ment où les bois peuvent être abattus et celui où ils peuvent être réalisés. Avec les mêmes bases d'évaluation que pour le précédent, nous arrivons, pour ce chantier, à l'estimation d'un capital nécessaire de 225.000 francs.

Avant la guerre, ces chiffres pouvaient être ramenés, respectivement, à 80 et 70.000 francs. Or, il est bien peu d'exploitants qui aient débuté avec un capital aussi important. Les tout premiers ont eu la chance de pouvoir prendre les chantiers les meilleurs parmi les plus faciles à exploiter; ils ont réussi, pour la plupart, à faire des fortunes honorables. Ceux qui sont venus ensuite ont moins bien réussi en général; il a fallu la période de hausse de 1920 pour 
leur permettre de se libérer des avances consenties et renouvelées indéfiniment par les acheteurs de bois de Grand-Bassam, moyennant passation de contrats pour la livraison (dans des conditions très désavantageuses pour les coupeurs) de la majeure partie, et même de toute la production de leurs chantiers. Ont-ils été assez heureux pour ne pas retomber, en 1921, dans la situation assez pénible où ils se débattaient prócédemment? Nous le leur souhaitons bien sincèrement.

Beaucoup de chantiers avaient été abandonnés pendant la guerre; les prospecteurs qui, bien inspirés, en recherchèrent dès 1919, purent donc trouver des zones intéressantes, très facilement exploitables. La hausse des bois se produisant, ces nouveaux venus ont pu gagner beaucoup d'argent; ils en ont, probablement, reperdu un peu depuis, mais ont été assez prudenis cependant pour ne pas tout risquer dans une extension inconsidérée de leur industrie. $\mathrm{Il}_{\mathrm{s}}$ se sont outillés, se sont fait réserver des zones assez étendues où ils pourront travailler dans de bonnes conditions pendant plusieurs années.

Actuellement, la situation n'est plus aussi avantageuse; si les cours de l'Acajou permettent encore d'exploiter avec profit, il faut, en tout cas, pour réussir, à ceux qui veulent se lancer à leur tour, une mise de fonds autrement importante. Les chiffres que nous avons indiqués (225-250.000 francs) sont des minima. Il reste bien quelques chantiers disséminés à prendre, peu éloignés des voies de rommunication et d'un accès relativement facile. Ils ne doivent pas, en tout cas, être très riches en $A$ cajou pour avoir été délaissés jusqu'à ce jour. Et dès l'instant qu' on désire entreprendre une exploitation durable, grouper plusieurs chantiers intéressants, i) faut s'avancer en dehors des zones fréquentées jusqu’à présent, et qui exigent pour l'évacuation des bois 10,15 et 20 kilomètres de voie Decauville. Les petits cnupeurs sont donc appelés à être remplacés peu à peu par des Sociétés à gros capitaux qui, seules, pourront engager les centaines de mille francs nécessaires à des exploitations nouvelles et courir les aléas que comportent de telles opératioins.

\section{Prix de REVIENT ET PRIX DE VENTE DES BOIS EXPLOITÉS}

A quel prix reviennent les bois obtenus par les exploitants? Il est bien difficile de répondre, même approximativement, à cette question. Ces prix peuvent varier, et varient en fait, suivant la situation des chantiers, suivant leur richesse, suivant l'outillage employé, suivant les capacités et l'activité de celui qui dirige le travail d'exploitation. Tel coupeur ou chef de chantier sortira 1.800 tonnes d'A cajou dans son année, alors que le chantier voisin, aussi riche et aussi bien situé, mais moins bien organisé, n'en produira que 300 ou 400 ! Et le premier n'aura pas employé beacoup plus de personnel que le second.

Tenons-nous en, pour notre démonstration. à des exploitants ni exceptionnellement qualifiés. ni trop médiocrement choisis, dirigeant des chantiers de valeur moyenne. Et disons qu'avec le personnel que nous avons indiqué au chapitre "Main-d'œuvre ", c'est-à-dire 125 à 130 hommes, bûcherons, manœuvres, cuisiniers. etc., il est possible de sortir de ces chantiers situés en première zone du chemin de fer ou d'une voie d'eau, et suivant qu'on dispose ou non de voie Decauville, de 800 à 1.200 tonnes de bois par an (1). en billes équarries, comprerant, d'après la proportion que nous a vons établie pour les essences exploitées. 910 d'Acajou et 1'l0 de Tiama, Iroko, Makoré, etc.. coupé à proximité du point de sortie ou des principaux chemins d'évacuation.

Dans les chantiers de deuxième et troisième zones, pour l'exploitation desquels le Decauville est à peu près indispensable, comptons 900 à 1.000 tonnes par an, suivant la longueur de roie

(1) Chiffre moyen. car sur un chantier de première zone bien conduit, il est admis qu on peut sorlir | lonne de bois par homme et par mois. soil $1 . f(00$ à 1.500 tonnes par an avec l"elfectif que nous indiquons. 
dont on dispose, suivant qu il faut établir ou non des relais pour l'évacuation des billes.

Voyons maintenant, quelles sommes sont dépensées pour obtenir ces différents tonnages :

Redevance d'exploitation pour un chantier

Frais de prospection, anterieurs à l'exploitation

Salaire du Chef de chantier

Salaire de deux commis indigenes.

Salaires et vivres de 30 bucherons a 3.50 I par jour en moyenne, pendant 300 jours

Salaires et vivres de 95 manouvres et chefs d'équipe, a 3 francs 1 par jour, en moyenne pendant 300 jours

Frais de recrutement des bûcherons et manouvres

Amortissement ou renouvellement du pelit maleriel d'exploitation.

$$
\text { Total }
$$

Mettons 150.000 francs, en chiffres ronds, somme à laquelle il y a lieu d'ajouter les frais d'amortissement $\left(12^{\circ}\right.$ o du prix de revient) du matériel Decauville employé. Cela peut représenter, suivant la longueur de la voie, de 10 à 40.000 francs par an. La somme totale dépensée sur ces chantiers variera donc, suivant leur situation et l'importance de l'outillage employé de 160.000 à 190.000 francs. Appliquons ces chiffres aux tonnages obtenus et nous verrons que le prix moyen des bois en billes équarries (2) sera compris entre 150 et 200 francs la tonne.

Ce n'est pas encore là le prix de revient des bois, port embarquement. L'exploitant a pu avoir à payer des frais de transport par chemin de fer ( 5 à 25 francs par toune); il a eu, en tous cas, à acquitter la taxe d'abatage ( 15 francs par mètre cube pour l'A cajou (3), 10 francs pour le Tiama et l'Iroko, 3 francs pour le Makoré et le Bossé). La densité pour l'A cajou en billes étant comptée à 0.700 environ, c'est donc 20 francs par tonne, 3 à 12 francs par tonne pour les autres essences.

(1) Coût moyen fin 1921, tenant compte des jours de repos, maladies, gratifications, etc.

(2) Certains exploitants laissen: une partie de leur production en grumes. Le prix de revient en est moindre. mais le prix de vente est également moins élevé.

(3) Nous avons vu plus haut que ces taxes sont fixées maintenant par arbre abattu et non par mètre cuhe. (Régime forestier.) qu il faut ajouter aux prix ci-dessus pour avoir le prix de revient. Notre prix de revient total, indépendamment des frais possibles de transport par chemin de fer, pour les bois qui ont à employer ce mode d'évacuation, sera donc compris entre 170 et 220 francs la tonne (bille équarrie), rendue quai ou plage point embarquement.

Nous ne faisons pas entrer en ligne de compte les frais d'entrepôt, de wharf ou de chargement, ainsi que les taxes de sortie qui généralement sont acquittées après que les bois ont déjà été vendus et font la différence entre le prix de vente quai port embarquement ou fob navire.

ll est bien entendu, et nous tenons essentiel-

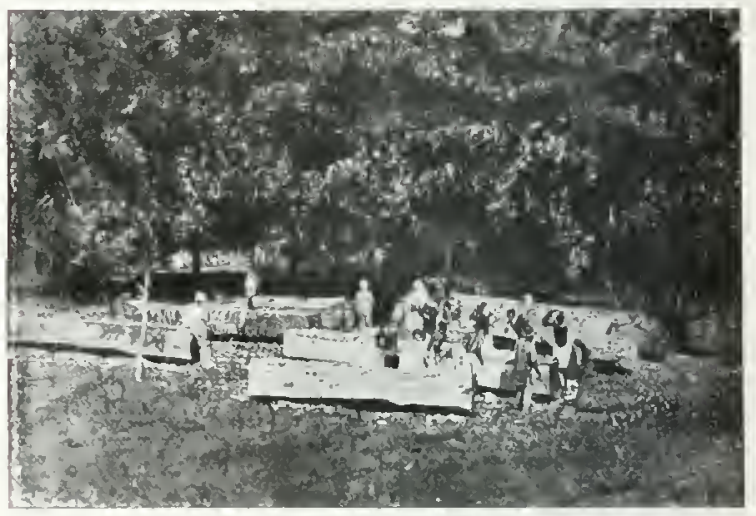

Equarrissage des billes.

Cliché Mélayer. - Grand-Bassam

lement à le faire remarquer, que ces chiffres de 170 à 220 francs la tonne, indiqués comme prix moyens de revient actuels des tois exploités dans les chanciers d'Acajou, ne sont que des bases d'évaluation. Ils peuvent, comme nous l'avons déjà dit, êrre inférieurs ou supérieurs à ces chiffres, suivant la valeur et l'emplacement des chantiers, suivant l'importance et le degré de perfectionnement de l'outillage employé, suivant l'organisation, suivant les capacités et l'activité de l'exploitant ou de son chef de chantier, suivant le coût des vivres pour la nourriture du personnel, etc. Une réduction de 0 fr. 50 par jour et par homme, lorsque les aliments diminuent de prix, permet de réaliser, en une année, une économie de plus de 20.000 francs sur un 


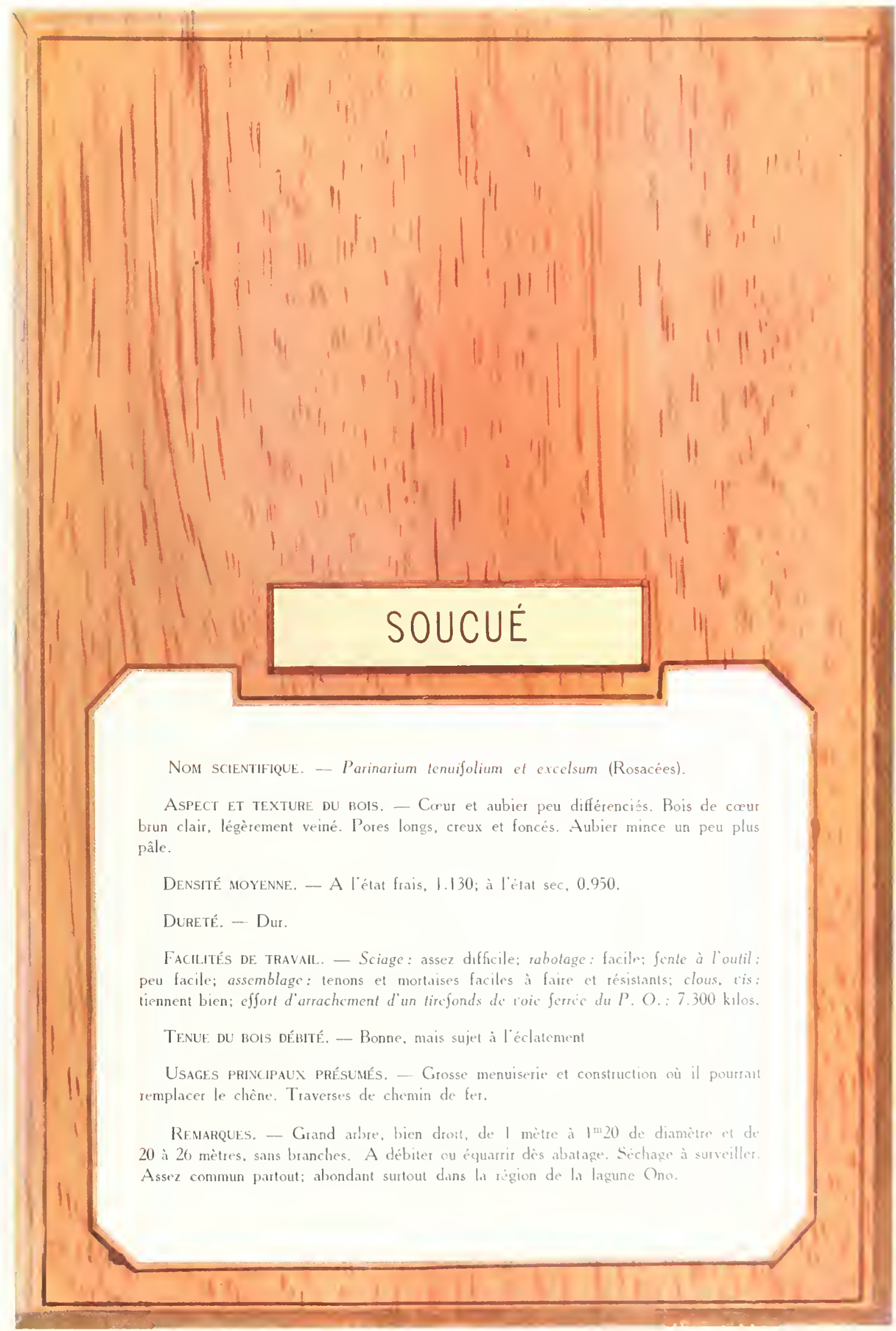


chantier, soit plus de 20 francs par tonne de bois obtenue.

Il en est de même en ce qui concerne la composition des essences abattues et la proportion d'A cajou; celles que nous avons indiquées n'ont rien d'absolu. Sur des chantiers très faciles à évacuer, on coupera davantage d'essences diverses: au lieu de faire I.000 tonnes dans l'année; on en fera peut-être 1.500, dont 400 ou $500 \mathrm{~d}^{\prime}$ Acajou, le reste en Iroko, Tiama, etc.. tandis que sur un chantier éloigné, ou desservi par un simple torrent d'hivernage, on s'en tiendra uniquement à l' $A$ cajou, les autres essences risquant de ne pas être vendues à un prix suffisant pour couvrir les frais de leur évacuation. Dans le premier cas, le prix de revient moyen sera incontestablement beaucoup moins élevé que dans le second; les bois seront également d'une valeur moyenne moindre.

Un exploitant bien outillé, qui fait travailler dans plusieurs chantiers à la fois, obtiendra d'autre part des résultats meilleurs si ses chantiers sont groupés et peuvent par exemple être desservis par une voie Decauville commune, que s'ils sont dispersés et nécessitent chacun un matériel complet d'exploitation.

Etablir un prix de revient pour les exploitations d'Acajou est donc un problème très complexe, étant donné la variabilité des conditions dans lesquelles ces exploitations peuvent être entreprises.

Il en sera de même pour les essences plus communes de menuiserie et de charpente. Toutefois, comme pour celles-ci il est d'ores et déjà possible d'exploiter 40 à $60 \%$ de la forêt au lieu de I o pour l'Acajou, et qu' on peut se cantonner pendant longtemps en bordure des voies d'eau permettant une évacuation permanente, le prix de revient sera bien inférieur à celui de l'A cajou et devrait pouvoir être ramené à un chiffre voisin de 40 francs la tonne enviror, soit de 28 à 40 francs le mètre cube, billes équarries, suivant la densité des essences dominantes des peuplements exploités: Bahia, Rikio, Dabéma, etc. Nous examinerons dans la troisième partie de ce tra- vail, le développement que peut prendre, à la Colonie, l'exploitation de ces bois.

\section{Prix de vente des bois exploités dans les chantiers d'Acajou.}

A quels prix sont maintenant écoulés les bois obtenus dans les chantiers? lci, nous avons des

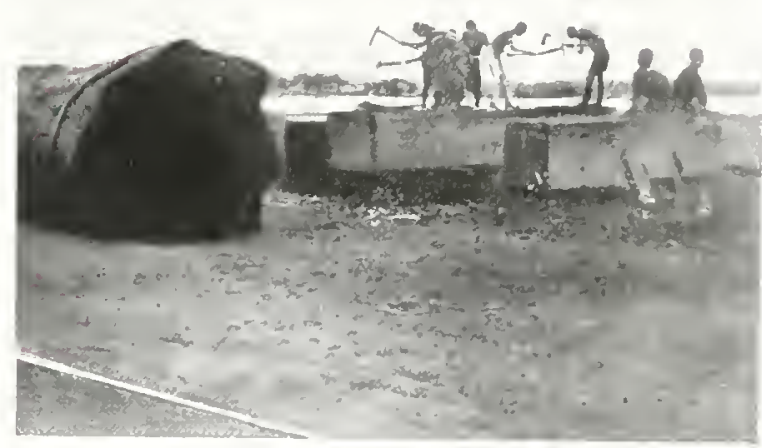

Herminetteurs au travail. Cliché Métayer. - Grand-Bassam

données plus précises et si nous ne pouvons prévoir quels seront les cours de l'avenir, nous pouvons du moins indiquer assez exactement les cours pratiqués à la Colonie mêrme, arant, pendant et depuis la guerre. Le diagramme ci-dessous en donnera une première idée; il se rapporte à l'A cajou et indique des prix pour des dimensions et qualités moyennes.

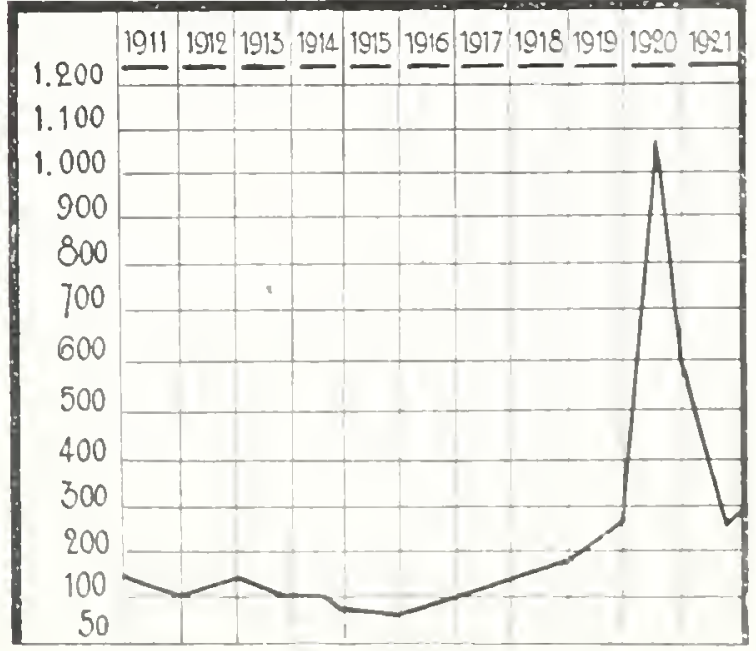


Ce diagramme fait ressortir la hausse surprenante dont l'A cajou fut l'objet en 1920. Les cours sont montés à 1.000 francs à Bassam pour les qualités moyennes en équarris, 1.100 francs et plus pour les beaux lots, tandis qu'on payait encore 800 et 850 francs les lots de billes ne faisant que 0.50 à 0,60 d'équarrissage; ce fut l'âge d'or pour les exploitants, ce le fut encore davantage pour les acheteurs intermédiaires, qui v'endirent jusqu'à 2.600 francs à New-York ou Liverpool ce qu'ils avaient payé 1.000 ou 1.100 francs à la Colonie. Le fret était cher, la difficulté d'expédier était grande, vu le manque de

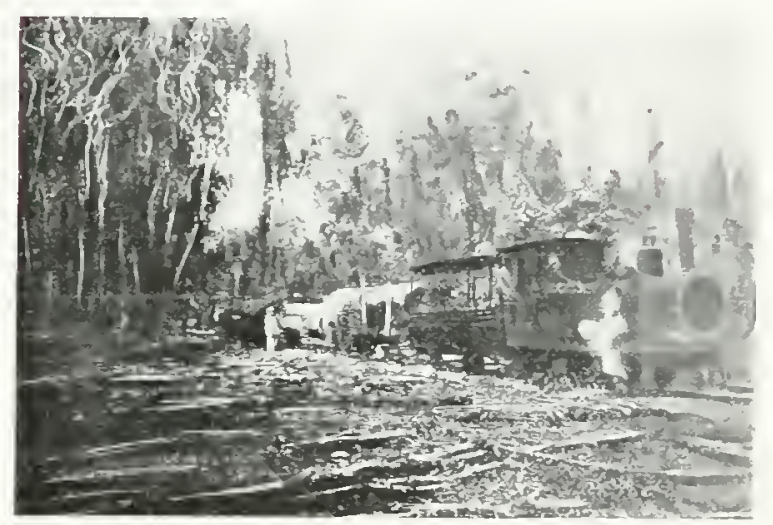

Train de billes.

(Cliche Metayer. - Grand-Bassam

bateaux. La marge de bénéfices était néanmoins telle qu'il en résulta une véritable spéculation. Les exploitants pouvaient se montrer exigeants; on allait leur faire des offres à domicile, on leur escomptait à l'avance la production de plusieurs mois. Que n'en ont-ils profité davantage! De juillet à octobre il se produisit un certain tassement des cours. En novembre, quelques marchés étaient encore passés à 800 et 850 francs, mais ce furent les derniers. Les offres se raréfièrent et les exploitants croyant à des manœuvres pour les obliger à baisser leurs prix voulurent maintenir leurs exigences; ce fut leur tort, car sans doute auraient-ils pu, à ce moment-là encore, passer des marchés importants à des prix avantageux, de 400 à 600 francs la tonne par exemple, pour des livraisons à échelonner sur 1921. Ce faisant. ils auraient été bien moins touchés par la crise. Car les cours allèrent s'affaiblissant et les acheteurs, devenus très circonspects, repoussèrent les cffres qui leur furent faites par la suite, l'écoulement de leurs propres stocks étant devenu difficile. La débâcle des cours se précipita; courant juin-juillet et août 192], de petits achats purent être faits aux exploitants les plus atteints par la crise, à des prix voisins de 200 francs la tonne; ces prix ne correspondaient pas aux cours pratiqués sur les marchés européens, lesquels atteignaient encore 500 à 600 francs et pouvaient permettre, étant donnée la baisse du fret, de payer de 250 à 300 francs à Bassam. Mais les transactions étaient presque nulles partout, les demandes très rares.

Les affaires reprirent légèrement en octobre, les cours de l'Acajou, qui avaient continué à baisser, se stabilisèrent alors en Europe et se maintinrent, jusqu'en fin 1921, entre 400 et 500 francs. Le marché est depuis ce moment un peu plus actif. Le fret est. d'autre part, de moins en moins cher. Bref, les prix offerts à Bassam depuis quelque mois se ressentent de la situation générale et se sont relevés sensiblement. Ils variaient fin décembre entre 240 et 300 francs suivant la qualité et la grosseur des billes. 11 est probable qu'ils ne redescendront guère au-dessous de ces cours, la production étant subordonnée aux avantages qu'ils laissent aux exploitants. Si les prix de vente devenaient inférieurs aux prix de revient la production s'arrêterait e! les cours remonteraient fatalement. C'est un peu ce qui s'est produit en 1921 : la presque impossibilité de vendre les bois obtenus a amené la fermeture des trois quarts des chantiers.

Avant la guerre. l'on n'avait jamais constaté de mouvements aussi brusques dans les prix. De 1911 à 1914, les cours avajent varié de 80 à 130 francs la tonne suivant la plus ou moins grande abondance des lots offerts aux acheteurs; les exploitants, dont le prix de revient des bois ne dépassait guère 60 francs la tonne, réalisaient 
un gain satisfaisant. Seuls, luttaient péniblement ceux qui avaient leurs chantiers desservis par des rivières secondaires à crues plus ou moins capricieuses.

Après la déclaration de guerre, les cours s'avilirent complètement. Il se vendit des lots à $50 \mathrm{fr}$. la tonne à Bassam ou Lahou. Quantité de billes furent abandonnées sur les chantiers et tout achetait déjà des quantités importantes à 140 et 150 francs.

\section{Autres essences d'ébénisterie exploitées.}

Les prix de vente de l'Acajou - Tiama et celui de l'Iroko, suivirent toujours d'assez près ceux de l'A cajou. On peut dire qu'ils sont généralement des quatre cinquièmes du prix de

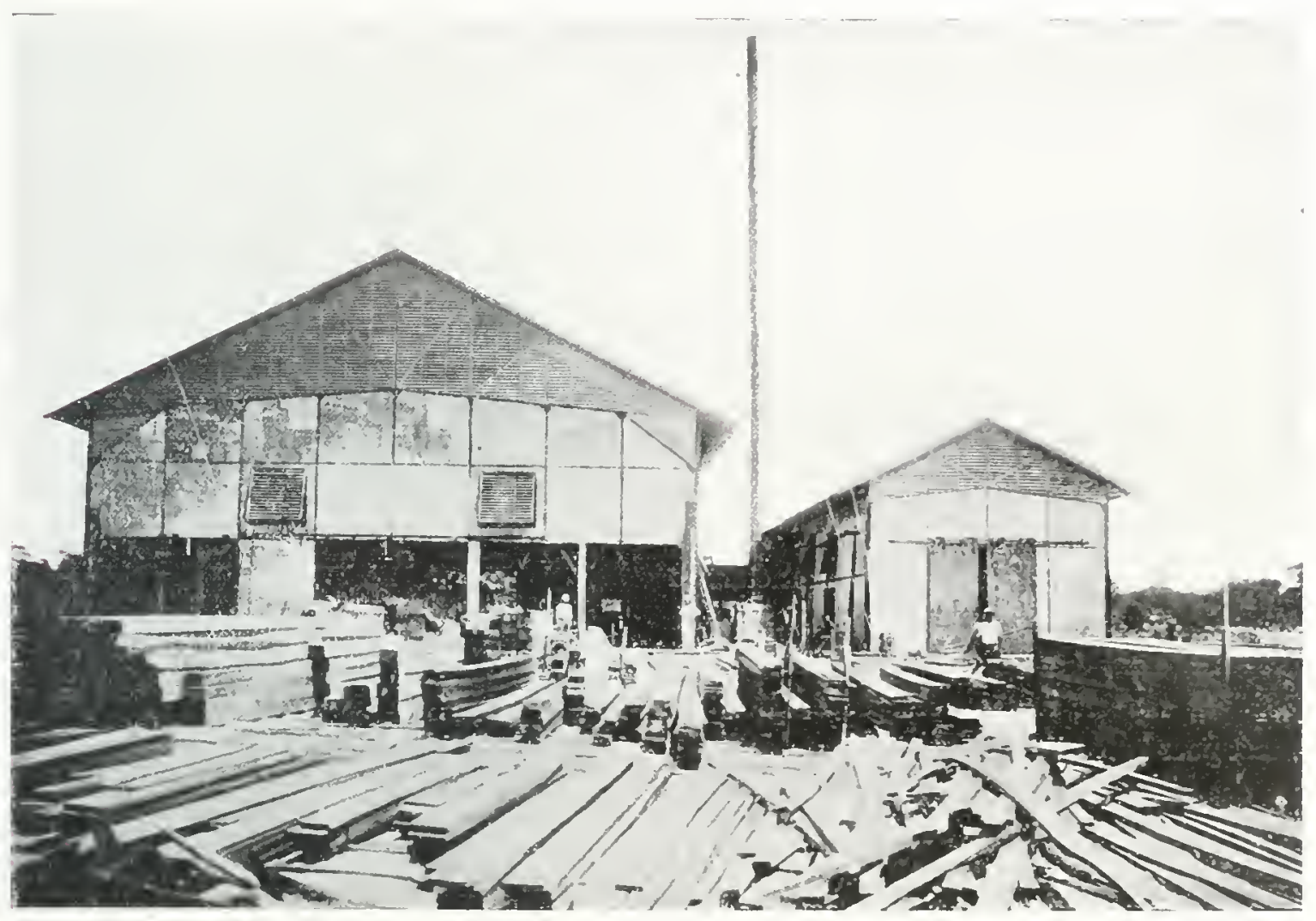

Scierie de la Compagnie forestiere.

le long des voies d'évacuation, soit que les exploitants eussent été mobilisés, soit qu 'ils n'eussent pas entrevu la possibilité de les réaliser à un prix couvrant les frais de tirage. Jusqu'à fin 1916 ce fut le marasme complet. Puis, peu à peu, quelques demandes d'achat se firent jour; on rechercha les Acajous de la Colonie pour la fabrication d'hélices d'avions; d'Angleterre. puis d'Amérique, les demandes arrivèrent plus nombreuses. Des chantiers se rouvrirent et l'on put vendre de nouveau à Bassam à 100 francs. ensuite à 120. Au moment de l'armistice, l'on
l'Acajou, à billes de dimensions et de qualités correspondantes.

Le Makoré et le Bossé se vendent meilleur marché, les trois cinquièmes à peu près du prix de l'Acajou. Le Badi et le Niangon dont il est coupé quelques centaines de tonnes chaque année, sont un peu moins appréciés encore et se vendent sur place, en temps normal. la moitié environ du prix de l'Acajou.

Il n'est exploité d'autres essences que pour l'alimentation des scieries locales. Nous verrons à l'un des chapitres suivants ce qui en est retiré. 


\section{Productions ET DÉBOUCHÉs.}

Le tableau ci-dessous donne le relevé, d'après les statistiques officielles, des exporiations de bois de la Côte d'Ivoire de 1901 à 1921 $\left(l^{\text {er }}\right.$ semestre).

\begin{tabular}{|c|c|c|c|}
\hline ANNEES & UNITES & ACAJOU & AUTRES ESSENCES \\
\hline 1909 & Tonnes & 15994 & \\
\hline 1910 & & 13.783 & \\
\hline 1911 & & $23 \quad 812$ & - \\
\hline 1912 & $\cdots$ & 30.490 & \\
\hline 1913 & - & 42652 & \\
\hline 1914 & - & 41049 & \\
\hline 1915 & - & 17867 & \\
\hline 1916 & - & 8134 & \\
\hline 1917 & - & 12817 & \\
\hline 1918 & Metres cubes & 36361 & \\
\hline 1919 & & 34961 & \\
\hline 1920 & 一 & $\begin{array}{ll}50 & 193\end{array}$ & 13.8911 \\
\hline 1921 & & 24631 & 2165 \\
\hline sem. & $\cdots$ & 24031 & $2 .+63$ \\
\hline
\end{tabular}

1 Ne sont pas comoris dons cer ch ftres les auelques cerlaines de metres cubes de bois scies expediés sur Konakr; et Dakar

A partir de 1918, les statistiquez sont établies au volume, alors qu'auparavarí elles l'étaient au tonnage. Mais il est facile de rétablir la comparaison par rapport aux années précédentes, en prenant pour base une densité moyenne de 0.700 pour des billes ayant déjà plusieurs mois de séchage. Nous obtenons par conséquent, pour les années $1918,1919,1920$ et 1921 ( $1^{\text {er }}$ semesire $)$ respectivement $25.452,24.472,37.619$ et 17.247 tonnes.

Avant la guerre, l'Allemagne était pour les bois un des bons clients de la Côte d'lvoire. Elle se partageait avec l'Angleterre près des deux tiers des $A$ cajous de la Colonie el achetait à peu près entièrement la production de Tiama et d'lroko. Disparu depuis 1914, le pavillon allemand recommence à flotter sur la Côte d'Afrique et depuis quelques mois on voit de nouveau, de temps à autre, des Woërman en rade de Bassam. La baisse de leur devise nationale (1) empêche momentanément les Allemands de procéder à des

(1) Novembre-décembre 1921. achats importants; mais il y a de gros besoins d'Acajou dans leur pays; que le mark remonte ou même se stabilise et l'on verra les demandes d'achat affluer.

La production de 1918, 1919 et 1920 est allée, pour les deux cinquièmes environ, en $\mathrm{An}$ gleterre. Les Etats-Unis ont acheté également des quantités importantes qui doivent approcher du cinquième des exportations totales; la France a absorbé un bon tiers des $A$ cc.jous et la majeure partie des autres bois exportés; le complément s'est réparti entre la Hollande, la Belgique, l'ltalie, etc. (1).

La majeure partie de ces bois est achetée sur place par des représentants des gros marchands de bois du Havre, New-York ou Liverpool. Certains exploitants préfèrent cependant (et le fait se pratique surtout dans les moments de crise) confier leur production à des maisons de commerce ou à des Banques qui leur consentent immédiatement des avances sur les lots et expédient ceux-ci sur les principaux ports européens où ils sont vendus, soit aux enchères publiques, soit à la commission. Quelques puissantes Sociétés se sont, d'autre part, organisées pour vendre ellesmêmes leur production en Europe et suppriment ainsi tout intermédiaire.

\section{Qualité des Acajous de la Cote d'Ivolre. - Leur emploi. - Tiama et Iroko.}

Sans être aussi beaux que ceux importés des Antilles, Cuba ou Tabasco, ou même du Brésil, les Acajous de la Côte d'Ivoire sont de très beaux bois d'ébénisterie ou de menuiserie de luxe. D'une belle couleur rose-saumon à l'état frais, ils se brunissent peu à peu à l'air et deviennent roussâtres; ils prennent très bien les teintures et le chromate de polasse et donnent ces jolis tons chauds qui les font tant apprécier. Leur grain est fin et régulier, très homogène;

(1) $22.368 \mathrm{~m}^{3}$ Acajou sur l'Angleterre en 1920; $9.834 \mathrm{~m}^{3}$ sur les Etats-Unis; $i 7.267 \mathrm{~m}^{3}$ sur la France; $703 \mathrm{~m}^{3}$ sur la Hollande. (Statistiques officielles de la Colonic.) 


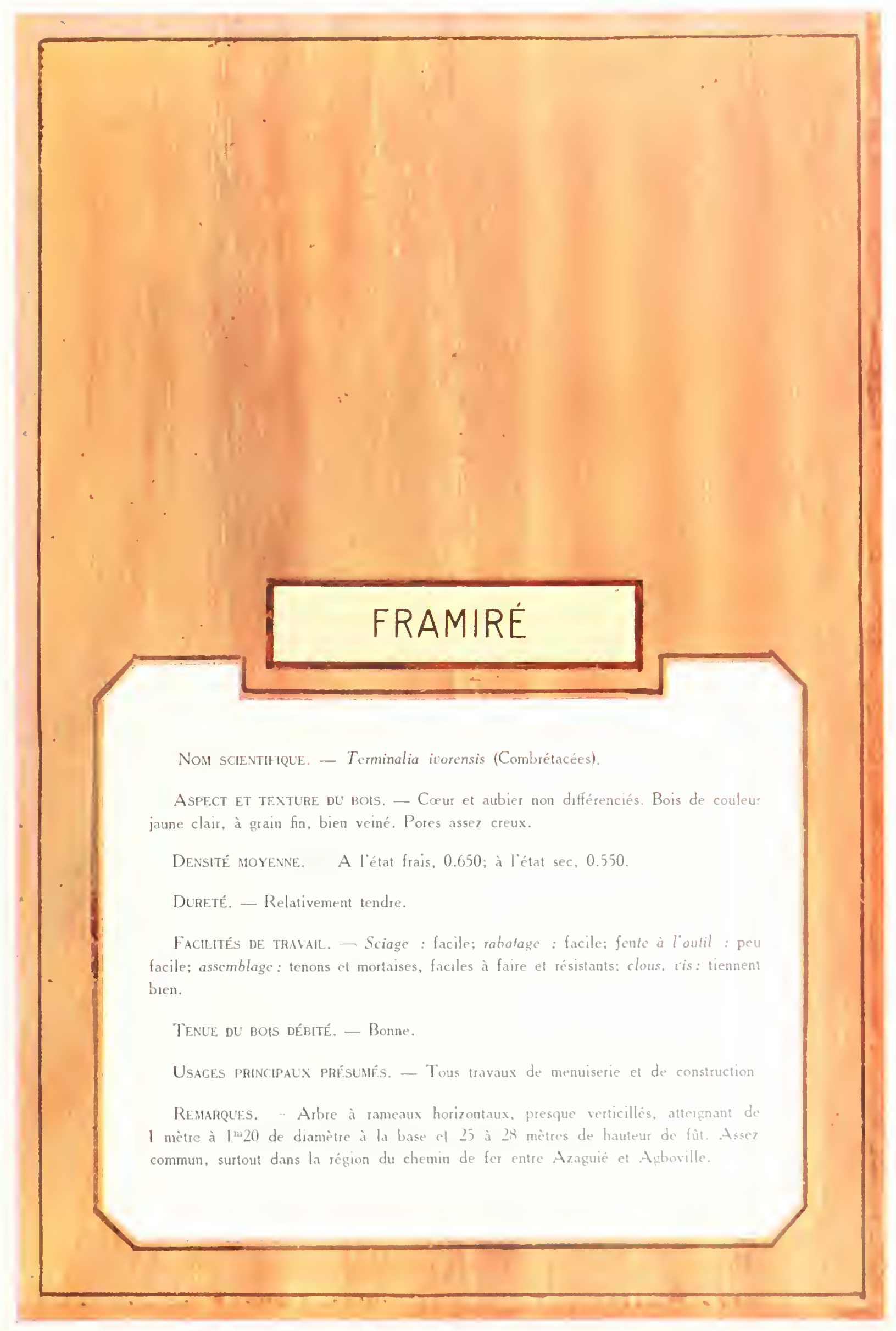



un peu à contre fil. suivant la laçon dont ils sont débités, ils présentent de jolis effetà satinés (1).

Mais ce qui donne beaucoup de valeur aux Acajous de la Colonie, c'est la dimension des billes qui font de $0^{\mathrm{m}}, 60$ à $1^{\mathrm{m}}, 30$, quelquefors plus, d'équarrissage, avec une moyenne de $0^{\text {mi }}, 80$ (la longueur est facultative; les fûts ont de 15 à 25 mètres et peuvent donner 3.4 .
Les industriels (on voii cela en France notamment) n'aiment pas beaucoup, d'une façon générale, employer des bois nouveaux. Or, les Acajous de la Côte d'lvoire sont des bois encore nouveaux, puisqu'il y a vingt ans seulement on n'en parlait nulle part. Les imposcr, malgré toutes leurs qualités, n’a pas été chose si facile qu'on pense ei nombre de marchands de bois

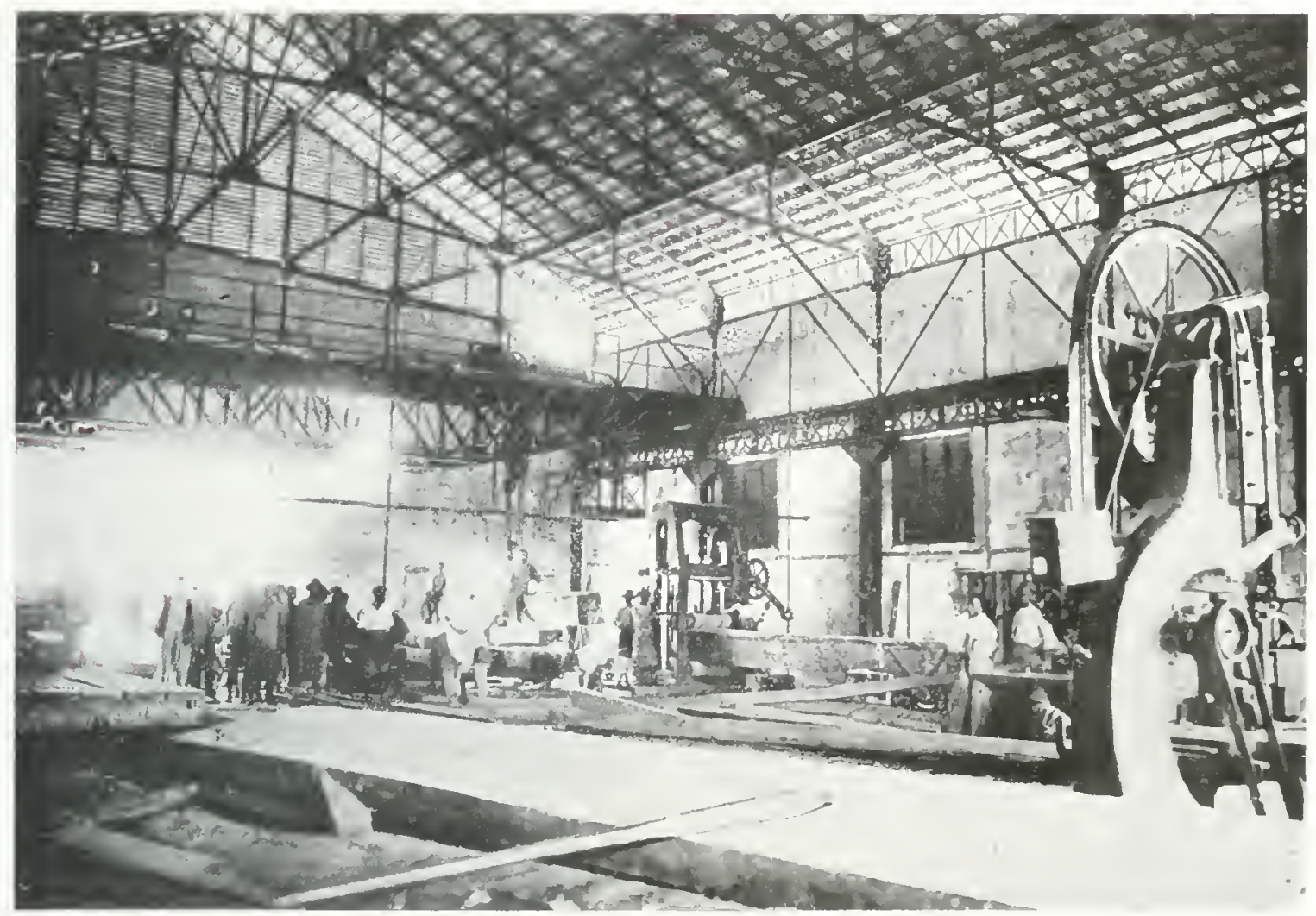

Scierie de la Compaznie forestuere.

Chiche Metaver -- Grand-Banam

5 billes de 4 mètres à 6 mètres). Ce sont des bois sans nœuds et absolument sains, produizant un minimum de déchet au sciage, tranchage ou déroulage.

Bien qu'ils fournissent près de la moitió de la consommation mondiale, les Acajous de la Côte d'Ivoire sont pourtant eñcore ma! connus.

(1) Près du littoral, ils sont toutefois moins beaux comme grain et comme couleur et rappelleraient, s'ils étaient tachelés de brun. les Acajous qu'on coupe en d'autres régions de la Côte d'Atrique. L'evploitation en est d'ailleurs presque mulle. ont dû, pour $\mathrm{y}$ arriver. user de supercherie. $11 \mathrm{~s}$ les ont présentés comme Acajou du Honduras, qualité dont ils se rapprochent beaucoup et qu'ils peuvent remplacer dans toutes les applications. Le procédé. déloỵal en sooi, a rćussi et réussit encore à l'occasion. La provenance américainc est en effet pour beaucoup d'emploveurs une garantie de bonne marchandise. Quoiqu'il en soit, nos Acajous de la Cöte d'Woire, dits Grand Bassam , conquièrent peu à peu les marchés et sont de plus en plus 
appréciés. On n'a plus besoin d'affirmer qu'ils viennent de l'autre côté de l'Océan pour les faire accepter.

Mais à leur tour, d'autres Colonies de la Côte d'Afrique envoient des Acajous en Europe et, comme ces bois sont de qualité inférieure à ceux de la Côte d'Ivoire, certains marchands, pour les écouler plus facilement et plus avantageusement, les baptisent, non pas $A$ cajous du Honduras, dont ils diffèrent trop, mais Acajous de Grand-Bassam, dont ils diffèrent autant, mais qui sont moins connus. Il en résulte pour ces derniers un discrédit sérieux. Il serait bon de veiller à ce que ces manœuvres ne se reproduisent pas trop souvent et de les dénoncer ouvertement lorsqu'on les découvre. Il y a bien un moyen, pour les employeurs, de s'assurer de la provenance des bois qui leur sont vendus comme "Grand-Bassam ", c'est d'exiger des vendeurs un certificat d'origine, ou une spécification timbrée au départ par la Douane (1), documents que les marchands de bois peuvent cbtenir très facilement des exploitants et dont ils peuvent faire établir et légaliser des extraits pour la vente au détail des lots reçus. Mais les employeurs songeront-ils jamais à contrôler de cette façon la provenance des Acajous qu'ils achètent?

Vendus moins cher que les provenances américaines, nos Acajous de la Côte d'Ivoire devraient être recherchés particulièrement pour le meuble en bois plein, pour la belle menuiserie d'intérieur (boiseries de salles de café, comptoirs, wagons, etc.), pour la fabrication d'hélices d'avions et de coques d'hydravion, pour la construction des bateaux de plaisance. Des billes assez longues seraient sans doute nécessaires pour ce dernier emploi, mais c'est là uniquement une question de préparation des bois,

(1) Nous apprenons au moment de mettre sous presse qu'un arrêté du Gouverneur de la Colonie, en date du 12 décembre 1921, vient de réglementer la délivrance de ces certificats. La question est donc tranchée. Nous somines heureux de constater que les observations que nous avions consignées à ce sujet, dans un rapport antérieur, ont été prises en considération. les exploitants pouvant livrer des billes de 8 et 10 mètres si on les leur demande. L'AcajouTiama pourrait également être utilisé pour cette industrie spéciale.

\section{Acajous de choix frisés, mouchetés ou "figurés"}

Qu'on nous permette de dire ici un mot des Acajous de choix, frisés, mouchetés ou plus ou moins moirés ou "figurés ", fournis également par la forêt de la Côte d'Ivoire et destinés à l'ébénisterie de luxe. Ces bois n'ont pas de cours établis, ni à la Colonie, ni en Europe; ils sont vendus plus ou moins cher, suivant la beauté $\mathrm{du}$ bois et suivant qu'ils sont plus ou moins' disputés des amateurs. Les bois frisés ou mouchetés, les plus communs, valent toujours deux ou trois fois le prix des Acajous ordinaires; les beaux moirés ou "figurés", peuvent valoir jusqu'à vingt fois ces mémes prix. On cite couramment à la Colonie le cas de trois belles billes "figurées " provenant d'un même arbre et dont les enchères à Liverpool atteignirent, avant la guerre, le chiffre de 105.000 francs. Vendues en 1920, ces trois mêmes billes auraient peut-être été adjugées 500.000 francs! C'est là, évidemment, un cas exceptionnel, mais il est assez. fréquent de voir vendre des Acajous de ce genre trois mille francs et plus, la tonne, quand les $A$ cajous communs sont cotés 500 ou 600 en France et en Angleterre.

Les effets frisés ou moirés produits sont dus à des ondulations plus ou moins fines, plus ou moin; régulières, des fibres du bois, ondulations qui sont cause des contre-fils souvent curieux constatés au débitage. A quoi sont dues ces ondulations? Le phénomène, s'il est constaté, reste à expliquer. Les arbres de cete sorte semblent être, scientifiquement, les mêmes que les autres, leur tronc est d'aspect absolument identique; ils paraissent aussi vigoureux que les autres et cependant les ondulations qui produisent les aspects moirés ou "figurés " étant presque toujours plus accentuées, près de l'aubier qu'au cœur même 
de l'arbre, pourraient bien être la conséquence d'une maladie quelconque dont souffrirait davantage l'arbre au fur et à mesure qu'il vieillit. Ce n'est là, en tout cas, qu'une hypothèse.

On trouve ces arbres un peu partout là où il y a de l'A cajou commun, plus fréquemment toutefois en certains endroits qu'en d'autres; ils restent néanmoins des exceptions, et les coupeurs ne doivent pas se baser sur leur découverte pour établir le budget de leur exploitation.

\section{Acajou-Tiama et Iroko.}

Des autres essences exploitées par les coupeurs, nous ne parlerons ici que des deux prin-

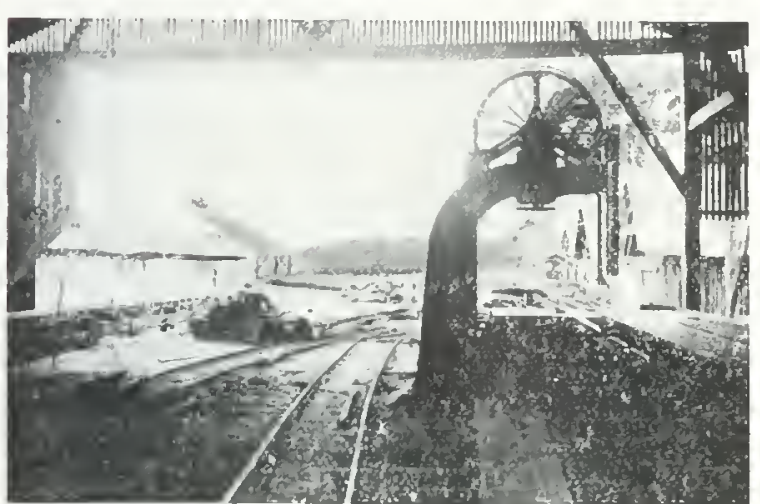

Scieries Africaines à Moossou. - Fond de la scierle. Scie P. L.

cipales, l'Acajou-Tiama et l'Iroko, nous réservant d'examiner, au Titre 111 , les caractéristique $_{S}$ des autres essences coupées occasionnellement et l'emploi dont elles sont susceptibles dans l'industrie. Seuls, avec l'Acajou ordinaire, le Tiama et l'Iroko sont un peu connus sur les marchés européens: seuls, ils ont donné lieu déjà à un mouvement d'exportation intéressant.

L'A cajou-Tiama est, scientifiquement, un Acajou. C'est un très bel arbre, qui dépasse peut-être, comme dimension, les autres $A$ cajous. Son bois est également de couleur rose saumon, mais plus ou moins foncée et parsemée de petits points noirs ou hachures qu'on dirait faites avec une aiguille. Ce sont ces minuscules taches noires qui font que le Tiama est moins apprécié que l'Acajou. En France et en Angleterre il est même très peu prisé. Par contre, ainsi que nous l'avons déjà signalé, les Allemands le recherchent volontiers et presque au même prix que l'Acajou commun. Ils apprécient particulièrement les Tiama foncés dénommés dans le commerce Tiama Sapelly.

Le Tiama pourrait être employé, au même titre que l'Acajou, pour la belle menuiserie d'intérieur, les wagons, les bateaux de plaisance, etc. Il est toutefois légèrement plus lourd que l'Acajou. Il y a également des Acajou-Tiama frisés ou moirés.

L'Iroko est un bois complètement différent. On le dénomme "teck africain", parce qu'il se rapproche, comme couleur, densité et qualité, du véritable teck. $\mathrm{C}^{\circ}$ est un très beau bois, jaune cognac au sciage, mais qui prend assez rapidement des tons vieil or et châtain par endroits, d'un très bel effet, et qui donnent aux meubles ou articles fabriqués avec ce bois un aspect d'ancien. Les Allemands l'emploient surtout dans la construciion des bateaux. Nous sommes certains qu' on pourrait l'utiliser avec succès dans l'ébénisterie.

Utilité pour les exploitants de ne présenter que des bois de toute première qualité.

Les exploitants ou, à leur place, les marchands intermédiaires, ont intérêt à n'exporter de la Colonie que des bois de tout premier choix. Ils les écoulent d'abord plus facilement: ensuite, ils contribuent, en agissant ainsi, à constituer une bonne renommée aux bois de la Colonie, à élargir leurs débouchés, à augmenter progressivement les cours auxquels ces bois sont réalisés par rapport aux bois similaires d'autres provenances.

11 est bien certain. en effet, que certains stocks, vieux de deux ou trois ans. piqués ou échauffés, fendus, achetés dans la fièvre de la spéculation en 1920, ont dù produire, sur les 
marchés où ils ont échoué, une impression plutôt délavorable. C'est ce qu'il faudrait éviter de renouveler par la suite, l'Administration locale dut-elle intervenir pour empêcher l'importation de bois aussi mauvais, bons tout au plus à faire du feu.

Les exploitants ne sauraient apporter trop de soins à la préparation et à la conservation de leur production, veiller à l'équarissage proprement fait; à défaut, écorçage immédiat des grumes, précaution indispensable pour éviter aux bois de se piquer, la sève montant presque sans arrêt au cours de l'année et aucune saison n'étant

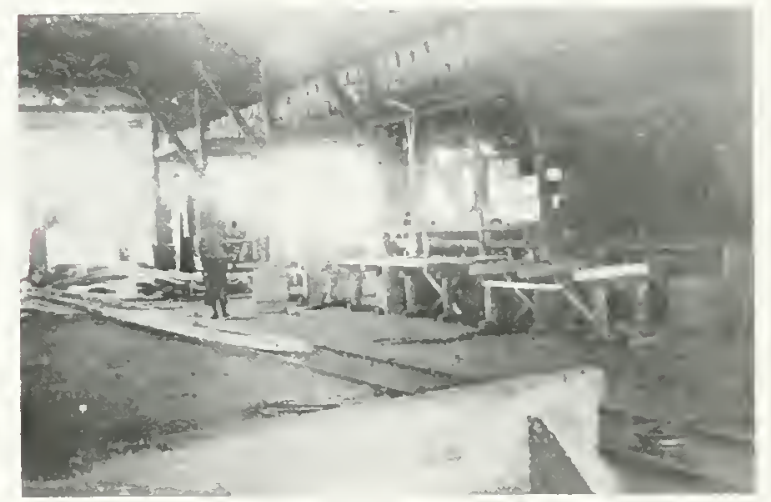

Scieries Africaines à Moossou. -- Ateliers d'affutage.

particulièrement favorable pour l'abatage. Certains exploitants enlèvent, quelques semaines à l'avance, aux arbres qu ils ont désignés pour l'abatage, une ceinture d'écorce pour arrêter ou tout au moins réduire, dans une certaine proportion, la circulaiion des sucs végétaux. C'est une pratique qui serait à généraliser pour la plupart des essences.

L'arbre, une fois abattu, ou les billes, après tronçonnage, devraient séjourner le moins possible sur le sol; si on ne peut les évacuer tout de suite, il est nécessaire de les isoler sur des bouts de bois placés en travers, qui permettent l'aération. Les billes, grumes ou équarris, doivent toujours être protégées le plus possible contre les ardeurs du soleil et conire les intempéries. Il est facile de les recouvrir de feuilles de pal- miers ou de branchages ou de leur construire des abris rustiques.

Il arrive, lorsque les billes attendent pour être évacuées sur le point d'embarquement ou, étant rendues en ce point, séjournent trop longtemps avant d'être expédiées, qu'elles éclatent ou se fendent en étoiles aux extrémités; c'est loujours la conséquence d'un manque de soins. Pour éviter les altérations de cette nature, les exploitants, lorsqu'ils le peuvent, mettent séjourner leurs bois en eau douce où ils se conservent parfaiiement pendant plusieurs mois. Cette opération a même pour résultat de préparer le séchage plus rapide des bois, l'eau dissolvant les gommes ou résines qui sont dans la matière ligneuse (1). 11 est regrettable qu'on ne puisse généraliser cette manière de faire, surtouť près des points d'embarquement; malheureusement l'eau de mer et, avec elle, les tarets, pénètrent dans les lagunes; la conservation des bois qui seraient immergés à proximité des points où ces lagunes commuriquent avec l'Océan paraît donc très douteuse.

\section{LES SCIERIES LOCALES. - LEUR ORGANISA- TION. - ECOUlEMENT DE LEUR PRODUC- TION.}

Il existe à la Colonie trois scieries (2) assez bien outillées dont le débit total devrait aiteindre 15 à 20.000 mères cubes de bois en grumes par an, soit, en bois débités, 12.000 à 15 mille mètres cubes. Deux sont installées à Moossou, en arrière de Grand-Bassam; la troisième fonctionne à Abidjan, au fond d'une des criques lagunaires qui entourent la ville. Leurs emplacements à toutes trois sont particulièrement bien choisis et leurs propriétaires se sont fait réserver des superficies de terrain assez vastes dans leurs environs immédiats, pour permettre le développement des usines et le stockage des bois.

(1) On ne peut toutefois l"appliquer aux bois très tendres qui pourriraient rapidement dans l'eau.

(2) Installées en 1917 et 1918. 


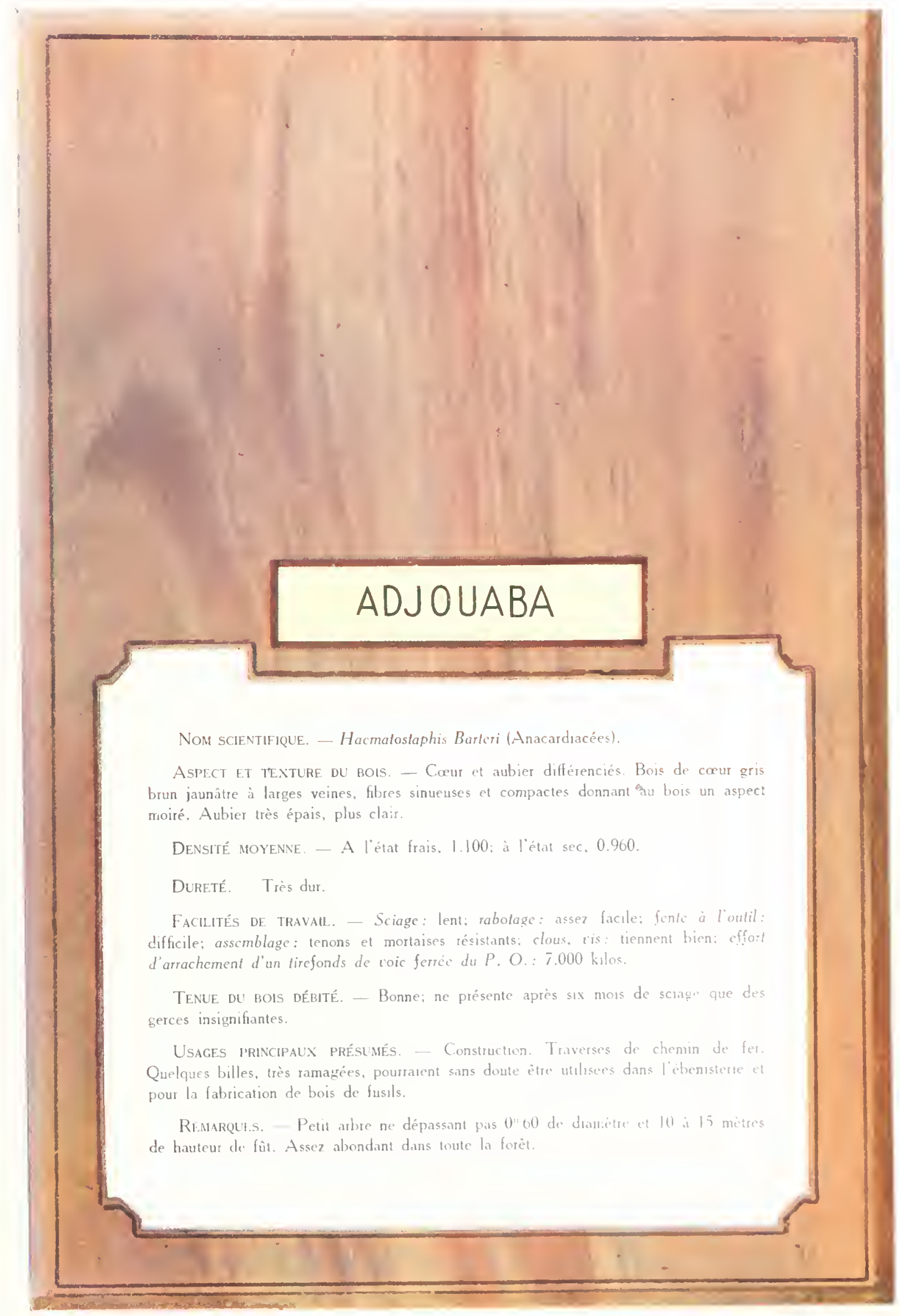


Les deux premières appartiennent à la Compagnie forestière de l'Afrique française et à la Société Vizioz et Cie (Scieries Africaines). Elles s'approvisionnent en bois d'œuvre sur les chantiers de la région du Comoë et des petites

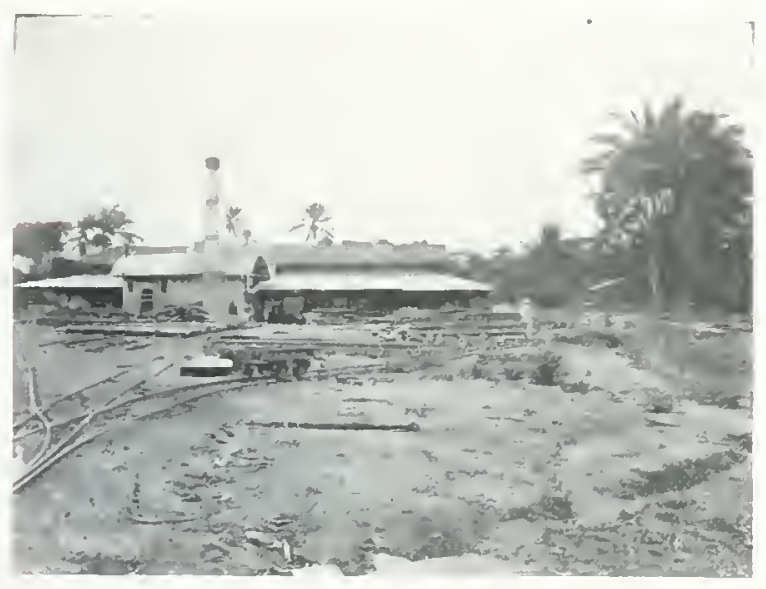

Scierie d"Hubert à Abidjan.

lagunes qui sont à droite et à gauche. Par le Hottage, elles reçoivent ces bois presque sans aucun frais. Celle d'Abidjan, qui appartient aux Etablissements Emile d'Hubert, est reliée par la voie ferrée aux chantiers de la Société, qui sont tous à proximité d'Abidjan, sur le côté Ouest du chemin de fer montant vers le Nord. Elle a l'avantage, sur ses concurrentes. de pouvoir recevoir, sans séchage préalable, les bois lourds dont elle a l'écoulement; le Dabéma, par exemple, qui flotte difficilement à l'étai vert.

Ces scieries, dont le débit est loin de correspondre aux besoins en bois de toutes sortes de l'Afrique occidentale, trouvent, sur place, à la Colonie même ou dans les Colonies voisines, des débouchés qui absorbent toute leur production. C'est fort heureux pour elles car, on peut le signaler sans esprit de critique, elles sont bien sommairement organisées et il est à peu près certain qu'elles devraient, ou changer de méthodes, ou fermer leurs portes, si elles en étaient réduites à l'éventualité d'exporicr les quelques milliers de mètres cubes de bois qu elles débitent chaque année.
Mais du fait que les besoins en bois sciés de l'Afrique occidentale françai:e dépassent de beaucoup les quantités pouvant être livrées par les scieries da la Côte d'Ivoire, ces dernières. vendent à l'usine ou quai Bassam, à des prix très avantageux, supérieurs, dans la majorité des cas, à ceux qu'elles obtiendraient en France, des bois qu' elles livrent au fur et à mesure qu'ils sont débités, sans séchage ou après séchage très insuffisant. Aucune qui dispose de hangars à toits étanches, indispensables à la Colonie pour obtenir une bonne conservation des sciages: aucune qui, débitant de 3 à 5.000 mètres cubes (I) de grumes par an ait, à un moment quelconque, 500 mètres cubes seulement do bois sciés disponibles, en bon état de conseriation. Tout est vendu d'avance; on travaille sur commande. Le bois est scié vert et il est livré ou amené vert à Bassam pour être embarquá. S'il peut être chargé assez rapidement sur un bateau de passage, enlevé dès débarquement à Konakry ou Dakar et mis à l'abri, il arrive à

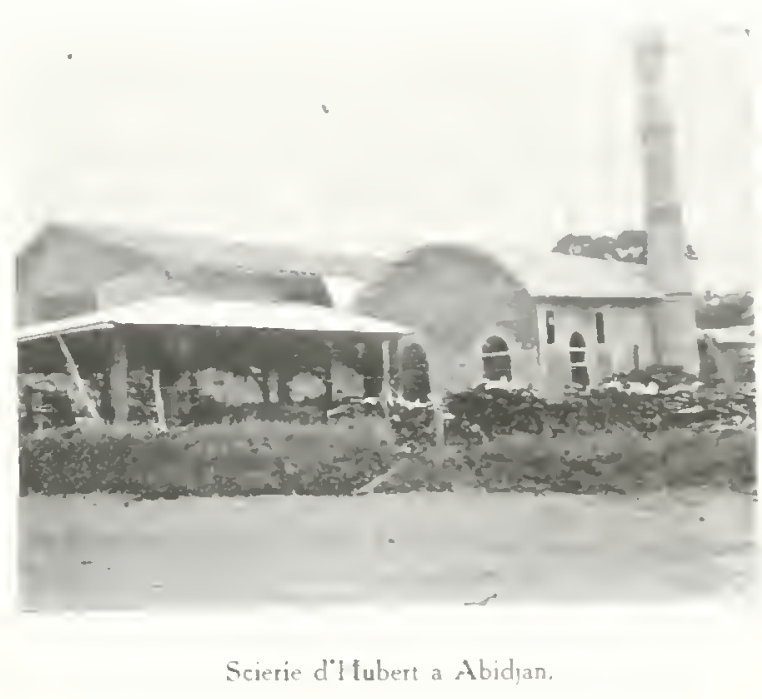

destination sans avoir trop subi de dommages: au destinataire de le faire sécher avant de l"em-

(1) Les scieries de la Compragnie torestière et celle des liablissements d'Hubert sont outillees pour débiter $500 \mathrm{~m}$ environ de hois en grumes par mois: celle de la société \izioz, 1.000) à $1.200 \mathrm{~m}$ : en tait, elles débitent beaucoup moins ef ne font guère plus d'un millier de mètres cubes par mois à elles trois. 
ployer. Mais si, par aventure (et cela arrive trop iréquemment), il doit séjourner sur les quais ou sur la plage avant embarquement ou après débarquement, sans être abrité, le soleil, le vent, la pluie lui font subir, en très peu de temps, un formidable déchet; les pièces se gauchissent, se tordent, les bouts éclatent, etc., les stocks deviennent pour $20,30 \%$ et plus, inutilisables. On conçoit que certains, parmi les destinataires du Sénégal, de la Guinée ou du Soudan aient pu faire, avec les bois reçus dans ces conditions, des expériences peu encourageantes et soient revenus aux vulgaires sapins ou pitchpins qui leur coûtent aussi cher, mais qui, au moins, ne leur donnent pas de semblables mécomptes (1).

Et pourtant les bois sciés à la Colonie se conservent, nous ne disons pas parfaitement, ce qui serait exagéré, car il y a sans doute plus de déchet qu'en Europe, mais dans de très bonnes conditions pour peu qu'ils soient mis à couvert (2). Nous avons vu les stocks emmagasinés

(1) Un rapport en date du 9 décembre 1921, adressé par $M$. le Gouverneur général au Ministère des Colonies, sur l'emploi des bois de la Côte d'lvoire en Afrique occidentale françaiśe, — sapport dont une copie est parvenue à l'Agence économique le 26 janvier dernier, c'est-à-dire au moment où nous allions mettre sous presse, - confirme entièrement notre point de vue.

(2) Les bois sèchent plus rapidement à la Colonie qu'en France; on peut les employer après dix-huit mois de stockage, alors qu'en France, il faut attendre de deusx à trois ans.

Il y aurait néanmoins avantage pour les scieries à disposer d'installations pour le séchage mécanique ou le dessévage des bois. Ce serait surtout utile pour les bois tendres qui ont une tendance à s'échauffer rapidement, même après débitage. C'est le cas pour l'Avodiré et le Bahia. Ce l'est encore plus pour le Fromager e: le Parasolier dont on pourrait tirer un parti très intéressant pour la caisse à bananes, pour la Guinée et les Canaries, et surtout la caisse à neufs, pour le Maroc. Ce dernier pays se procure à très haut prix, en Portugal et en France, la planche nécessaire aux emballages d'ceufs et encore cette planche, qui est de pin ou de sapin, n'a-t-elle pas la souplesse que présente celle du Fromager.

Les procédés de séchage ou de dessévage sont nombreux. Nous n'entrerons pas dans leur détail, d’autant plus qu'il serait délicat d'en conseiller aucun avant de l'avoir expérimenté sur place, les conditions climatériques locales constituant un facteur dont il y a lieu de tenir le plus grand compte. C'est une question à éludier et à mettre au point. à Moossou par la Mission des Bois coloniaux, stocks achetés aux scieries en vertu de contrats (1) passés pendant la guerre, et conservés pendant plus de deux ans, sous des hangars à toits de planches disjointes sur lesquelles il n'avait pas été placé de couvre-joints. L'eau de pluie ruisselait dans les interstices et les gouttières traversaient les piles de madriers et planches qui étaient en dessous. Lorsqu'on défit les piles il n'y eut cependant guère plus d'un cinquième des bois qui furent mis au rebut; tout le reste était en fort bon état, quoique légèrement noirci par l'humidité et le voisinage de la mer. Un lot, cédé à un commerçant de Bassam, fut revendu en France à raison de 1.600 francs le mètre cube! (C'était en 1920.) Tout le stock cut pu être vendu très facilement en France, et surtout à Dakar, s'il n'y avait pas eu à GrandBassam impossibilité presque absolue, à ce moment-là, d'expédier des bois sciés. Les capitaines de bateaux se refusaient à les prendre, trouvant, plus qu'il ne leur en fallait, de fret plus avantageux. Il est bon d'ajouter que c'est précisément en raison des difficultés de le faire transporter, que la Mission des Bois coloniaux avait décidé de réaliser ce qui restait de son stock sur place, plutôt que de l'expédier en France comme il avait été prévu.

Chacune des trois scieries dispose de scies alternatives verticales et horizontales et de grosses scies à ruban pour débiter les grumes, de scies à ruban plus petites et de scies circulaires pour refendre ensuite les plateaux obtenus. Elles débitent des traverses de chemin de fer pour le

(1) Ces contrats portaient sur plusieurs centaines de mille tonnes que le Ministère des Reconstructions Industrielles comptait importer en France pour les Régions dévastées. Ils avaient été conclus en 1917 et 1918 à des prix qui permettaient $(60$ à 70 francs le mètre cube) la concurrence avec les bois du Nord. Quelques milliers de mètres cubes furent livrés en 1918 et au commencement de 1919, mais la hausse des bois se produisant, et avec elle celle de la maind'œuvre, de l'outillage, e'c., les fournisseurs durent, sous peine de perdre beaucoup d'argent, demander la résiliation de leurs contrats. Elle leur fut accordée sans trop de difficultés. 
Sénégal et des bois de construction, charpentc et menuiserie pour les différentes Colonies.

Quelles sont maintenant les essences qui les alimentent? Avodiré, Bahia, Dabéma, constituent les trois principales, puis Bodioa, Sougué, Bossé, Niangon, Fraké, Rikio, toutes de bois excellent, qu'elles trouvent en quantités plus ou moins grandes sur des chantiers faciles à desservir et qui reviennent tout au plus à l'usine (bois en grumes) de 30 à 50 francs le mètre cube (suivant densité).

Vendant couramment de 180 à 280 francs le mètre cube, suivant les essences et le sciage de-

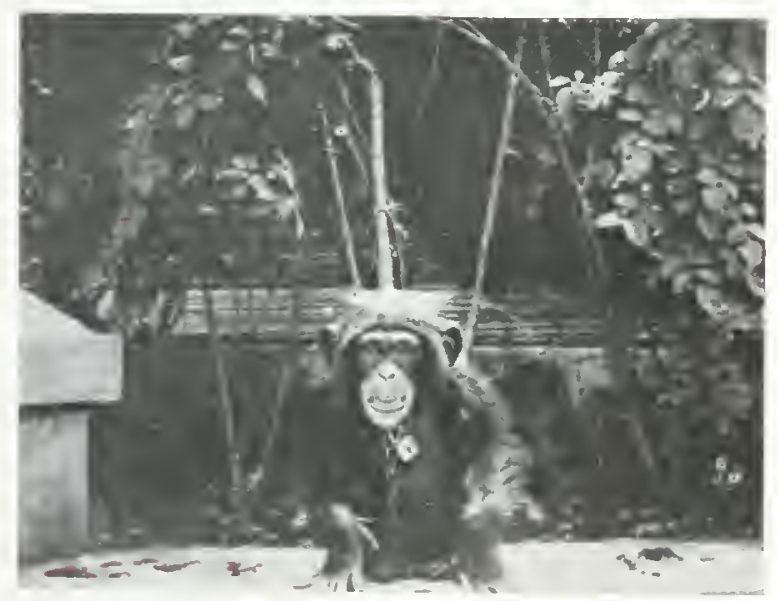

Jeune chimisanze.
Cliché Burger

mandé, les scieries, en tenant compte des déchets au sciage et des capitaux que nécessite leur installation, ne sont donc pas une mauvalic affaire pour leurs propriétaires. Malheureusement, et c'est sans doute la raison de leur ron-extension, elles exigent pour travaille dans de bonnes conditions, un personnel spécialisé assez difficile à recruter. Les réparations ne sont pas non plus toujours faciles à faire sur place: les pièces de rechange arrivent avec du retard. Bref, les scieries, à la Colonie, ne marchent pas toujours très régulièrement. Il $y$ a néanmoins lieu de présumer qu'elles s'organiseront peu à peu, aussi bien à ce point de vue qu'à celui du séchage des bois, et pourront se développer sans avoir à redouter la mćvente de leur production.
L'Afrique occidentale française (ports de Dakar, Saint-Louis, Konakry et Porto-Novo) importe encore annuellement, de France et de l'étranger, malgré l'arrêt momentané des travaux et des constructions, 6 à 8.000 mètres cubes de sapin, pitchpin et bois divers, bois qui sont vendus beaucoup plus cher qu'en Europe et que peuvent concurrencer, que doivent méme évincer complètement les bois sciés à la Côte d'Ivoire (1). Et puis, il y a le Maroc (2) et l'Algérie qui importent des quantités bien plus importantes encore et où les bois de la Côte d’Ivoire peuvent pénétrer peu à peu. Pour ces

(I) Nous regrettons de ne pouvoir citer les chiffres exacts des importations de bois de la Côte d'Troire, au Sénégal, en Guinée et au Dahomes; les statistiques officielles n'en font pas mention, ne relevant pas le trafic qui se fait entre les différentes Colonies du groupe de l'Afrique occidentale. On peut estimer que ces importations, qui vont samplifiant, ont dû atteindre 2 à 3.000 mètres cubes pour les années 1920 et 1921 .

500 à 600 mètres cubes de plateaux ont également été importés en France en 1921.

(2) Des ranseignements publiés assez récemment par l'Office du Mlaroc. il ressort que le Protectorat, zone française, importait, arant guerre, une movenne de 45.000 tonnes de bois divers, dont près de to not tonnes de pins et sapins sciés, et le reste en bois durs. L'importation s'est ralentie pendant la guerre: mais. dès 1919, elle reprenait avec 18.000 tonnes et approchait, en 1920, des chiffres d'avant guerre (40.000 t.).

Les pins et sapins sciés tiennent encore, en 1920. la première place, avec 27.600 tonnes. La proportion des autres bois a néanmoins augmenté sensiblement par rappor: aux années 1912-1913. Elle a été. pour la dernière année connue, de 12.600 tonnes. dont 8.800 tonnes de traverses de chemin de fer. 3.000 tonnes de chêne brut ou équarri. 800 tonnes de bois divers, sciés pour le charronnage. la menuiserie et l'ébènisterie.

La zone espagnole a importé, de son côté, en 1920. 18.000 ronnes de bois divers, dont 7.000 de bois autres que des pins et sapins.

Les pri sur place étaient à peu près les suirants. au début de l'année 1921 : sapin blanc, 600 francs le mètre cube: sapin rouge, 750 francs; en bois sciés. en grumes ou équarris. pin des Landes. tio trancs: pitchpin. 800 francs: chene. 950 francs: nover. 1.200 francs: platane. 700 trancs: Ofoune, 1.j(M) tr.: peuplicr, 550 francs. Ces prix ont certainement baissé pas mal depuis. Neanmoins. ils doivent encore ètre assez éleves pour permettre aux bois de la Côte d'Troire de concurrencer avec succies. à Casablanca et dans les autres ports du Maroc. les twois importes de Vorvege ou de France, qui ont à supporter des trais élevés de transport. 
derniers pays, comme pour le Sénégal et les autres Colonies de l'Afrique occidentale française, le facteur transport maritime joue en faveur des bois de la Colonie et telles catégories de bois, la traverse de chemin de fer par exemple, qui ne seraient pas "payantes " (1) s`il fallait les exporter en Europe, le deviennent dès l'instant qu'on les expédie dans un pays plus rapproché de leur lieu d'origine.

(i) Nous examinerons cette question plus loin.
Mais il y a mieux à faire pour les bois do la Côte d'lvoire que de conquérir le marché lc.:al et même les marchés de l'Afrique du Nord. Ils n'auraient qu'un mérite relatif à obtenir ce résultat. C'est le marché français, lui-même, qu'ils doivent libérer, tout au moins dans une certaine mesure, des importations de bois étrangers. Nous allons examiner, dans ce qui va suivre, si la chose est faisable et dans quelles conditions il paraît possible de la réaliser.

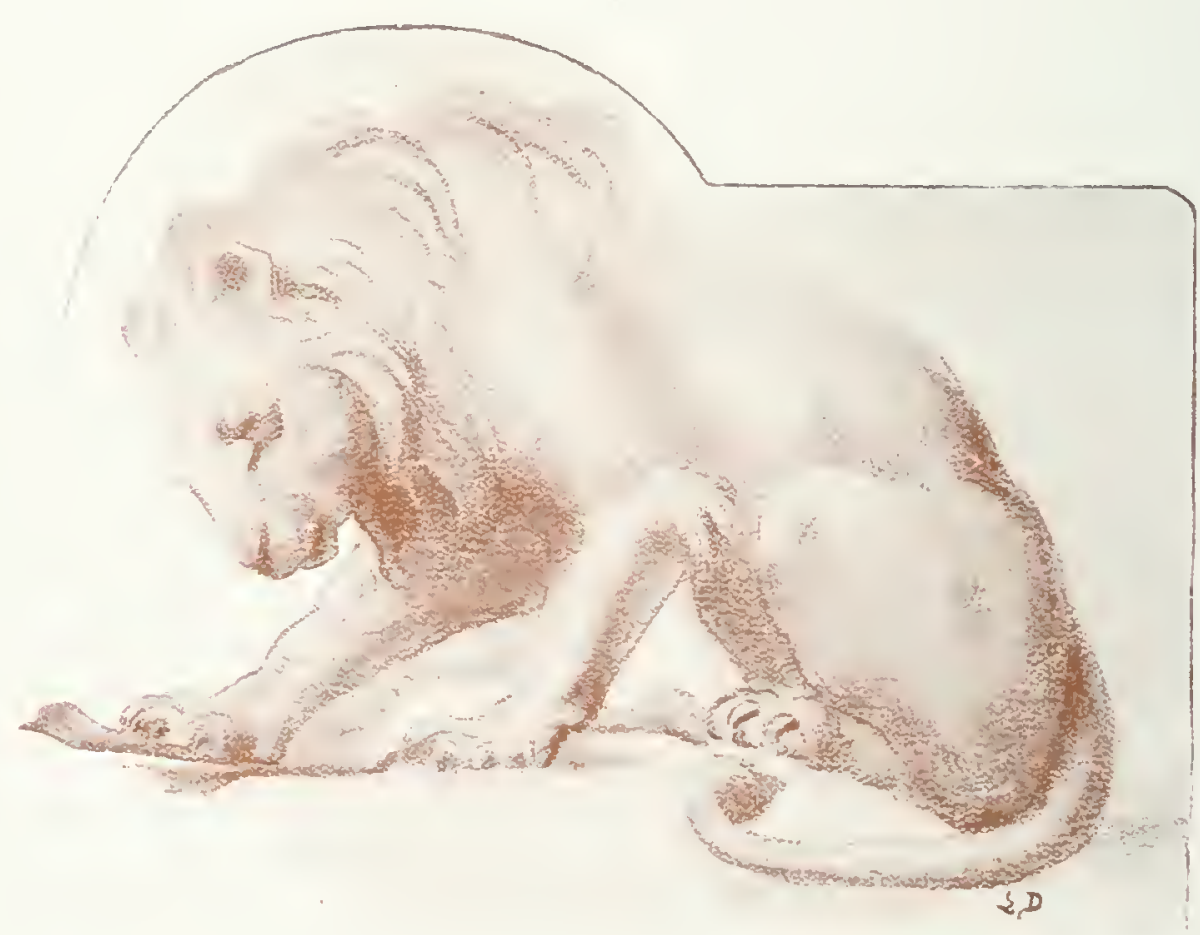




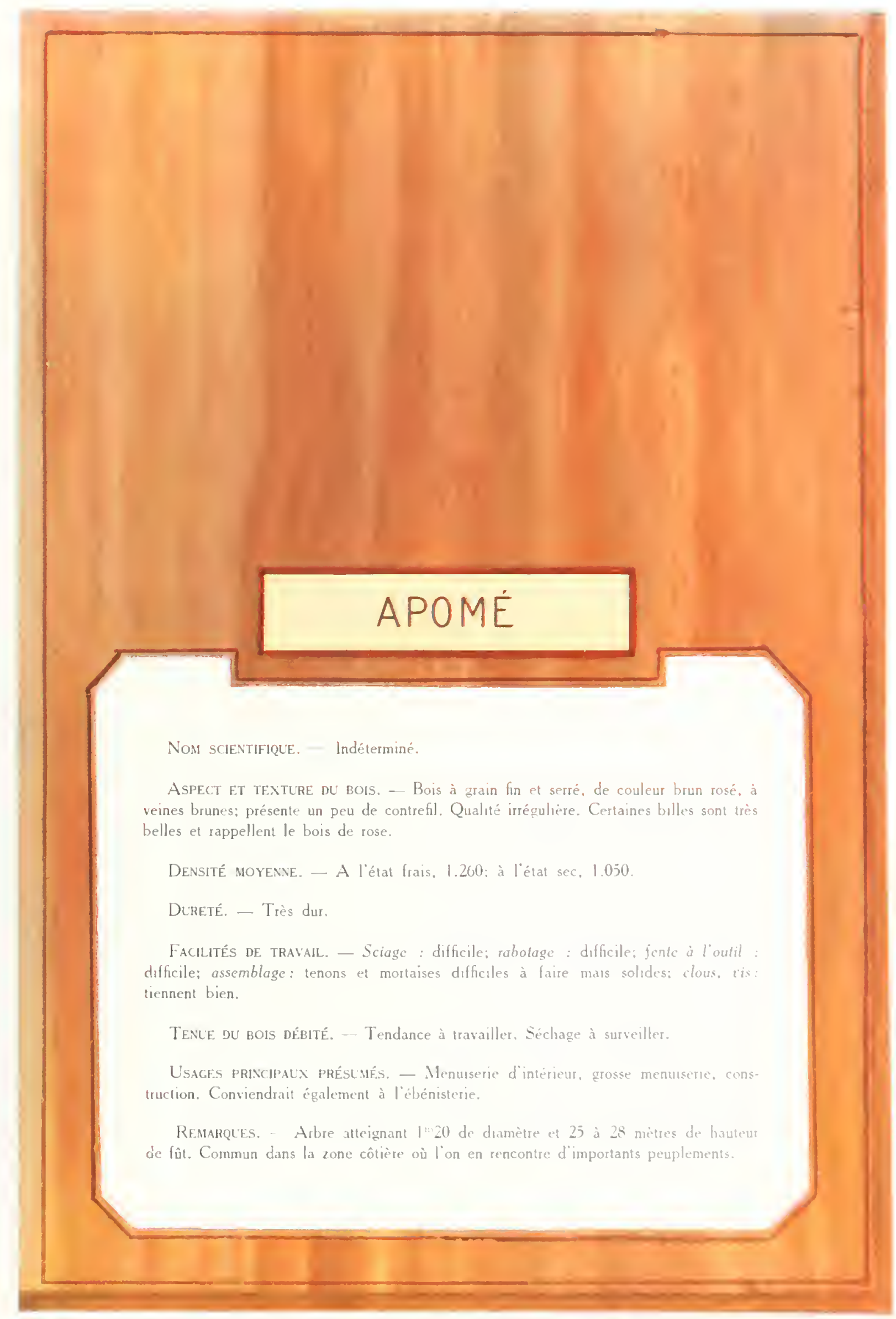





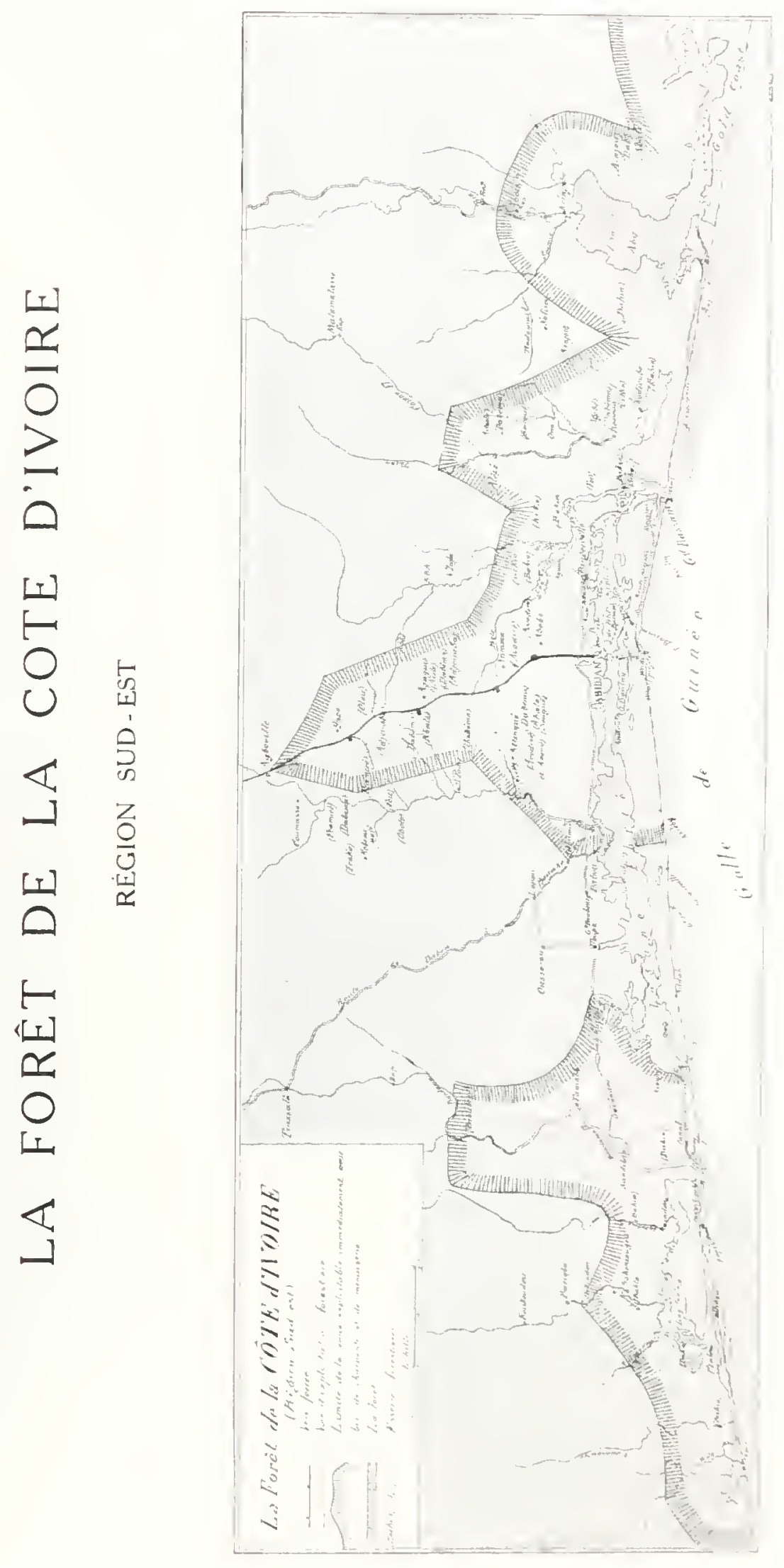





\author{
TITRE III
}

\title{
Avenir réservé aux bois communs Leur exploitation intensive et leur importation en France
}

\section{SOMMIAIRE}

Écoulement en Europe des bois de la Colonic autres que les bois d'ébénisterie de grand choix. - I'aleur des hois coloniaux en général - Essences à exploiter à la Côte d'Tuire et à importer en France. - Présentation des hois; sciage et stockage. - Prix de revient et prix de vente possible des bois importés de la Colonie.

Ecoulement et propagande: intervention de l'État. Comment organiser l'exploitation des bois communs. 


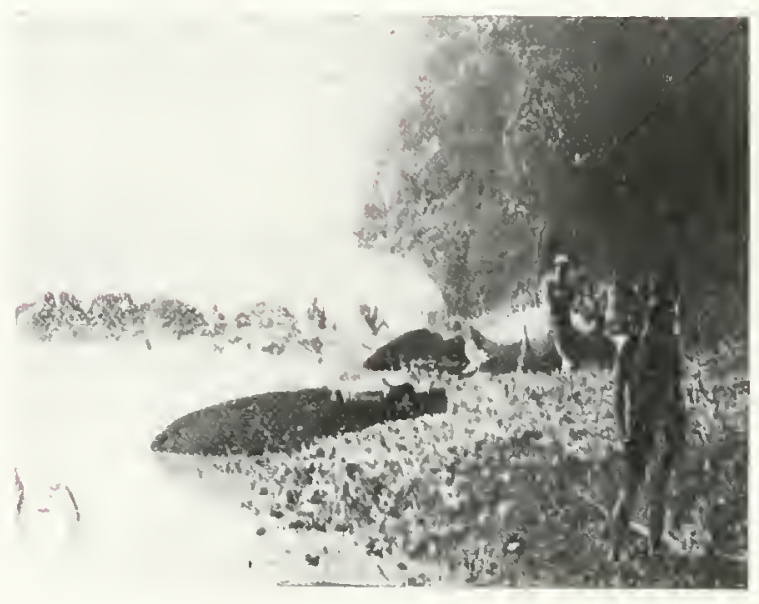




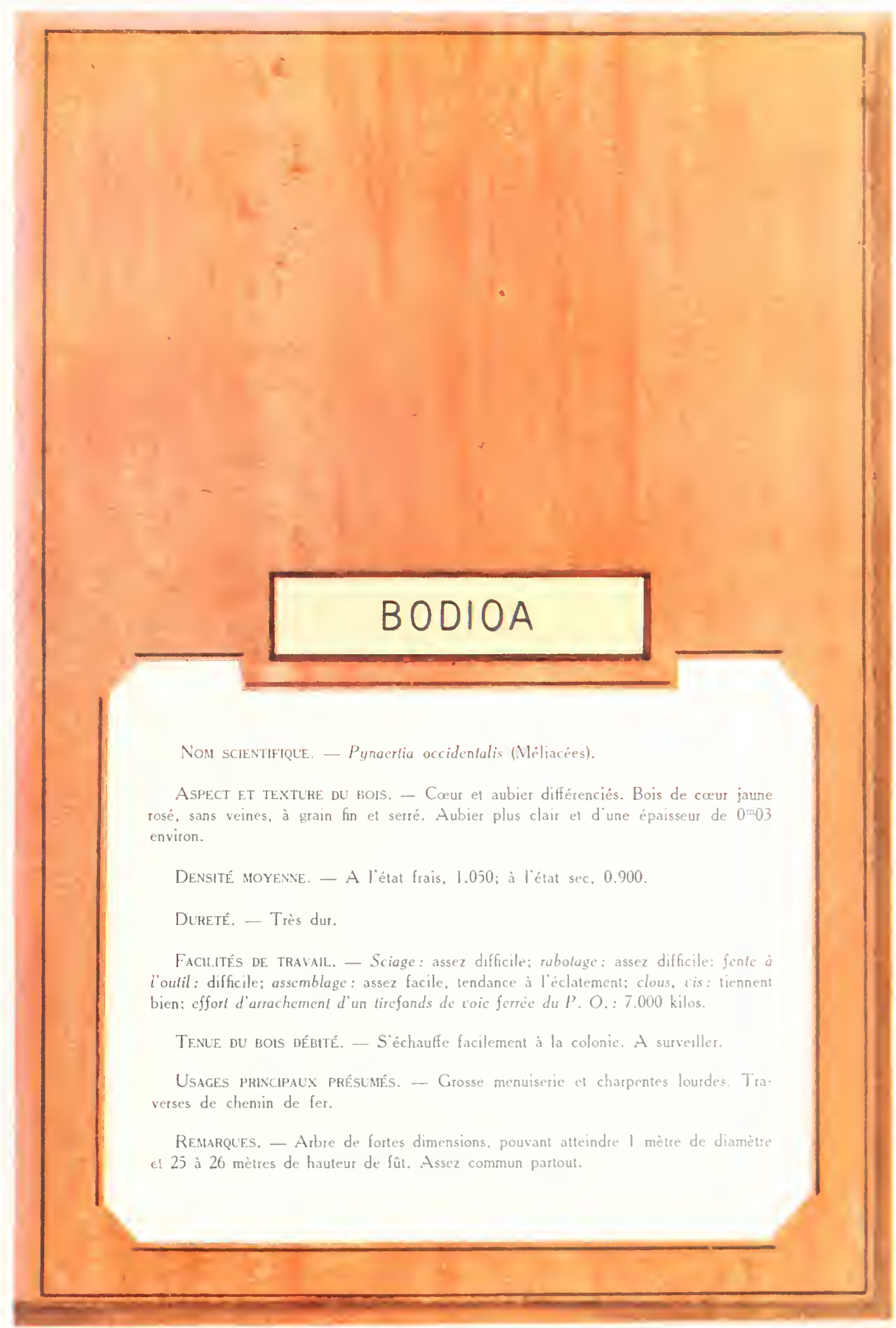




\section{Avenir réservé aux bois communs. - Leur exploitation intensive et leur importation en France}

\section{ECOULEMENT EN EUROPE DES BOIS DE LA Colonie autres QuE LES BOIS D'ÉBÉNTSTERIE ET DE GRAND CHOIX}

Nous avons vu, dans les chapitres précédents, que, malgré toutes ses richesses forestières, la Côte d'Ivoire n'exportait guère, en fait, que de l'Acajou et quelques centaines de tonnes de Tiama et d'Iroķo, malgré les besoins énormes en bois de ioutes sortes résultant de la guerre et des ruines qu'elle a accumulées chez nous et nos. alliés. L'exploitation des essences autres que celles précitées, dont bon nombre doivent être cependant d'un écoulement facile et peuvent remplacer nos meilleurs bois français, n'a encore pris, à la Côte d'Ivoire, qu'un développement embryonnaire, la production ne correspondant même pas aux besoins locaux de l'Afrique occidentale française.

Pourtant notre pays, à lui seul, importe de l'étranger des quantités considérables de bois. Avant la guerre, c'éiait 3 millions à 3 millions 500.000 mètres cubes qu'il nous fallait importer chaque année (1). Avec l'exploitation inten-

(1) Sur une consommation totale de 11 à 12 millions de mètres cubes, dont 6.000 .000 de mètres cubes de bois de sciage et d'industrie. (Années 1912-1913.)

L'Algérie et la Tunisie importaient avant la guerre de 200 à 250.000 tonnes de hois. Ces chiffres sont tombés à 52.000 tonnes en 1919 et 105.000 tonnes en 1920 . sive dont nos forêts ont été l'objet pendant la guerre, avec les besoins considérables qui surgissaient après la retraite des Allemands dans les régions envahies, on présumait que l'importation des bois étrangers allait croître immédiatement dans des proportions considérables, atteindrait facilement et dépasserait peut-être 8.000.000 de tonnes (1). Or, šil est incontestable que nos forêts sont très appaurries et que nos besoins devraient, d'autre part, être plus grands qu'avant la guerre, les importaiions de bois sont pourtant loin d'avoir pris l'accroissement prévu (2). Il faut dire, toutefois, qu'au lendemain de l'armistice. on s'est trouré en présence de stocks considérables, entassés pour les besoins de l'armée, et qu'on a pu emploỵer à des constructions provisoires diverses. Il faut dire également que les travaux de reconstruction des régions libérées n'ont été menés que bien lentement en 1919 et 1920 . II $y$ en a pour dix ans à les achever. D'autre part, la construction. dans tout le reste de la France. d'immeubles à usage d'habitations, suspendue depuis 191 f. n’a pas encore repris à cause de la cherté de tous les matériaux, du coût de la main-d'curre. II faudra bien! venir. l"insuffisance marquée de

(1) Bertix, Revue Colonics et Marine janvie: 1020. Divers autres auteurs.

(2) $8+1.000$ tonnes seulement de hois d"ceure. bruts ou débités en 1919:1.310.000 en 10.0. 
logements se faisant sentir à Paris et dans toutes les villes de quelque importance. La consommation de bois d'œuvre ne peut donc aller qu'en augmentant, pendant une quinzaine d'années. Elle diminuera sans doute par la suite, mais il est à prévoir qu'elle restera en tout cas supérieure à ce qu'elle était avant la guerre.

Or, au prix où sont actuellement les bois de toutes sortes, on peut estimer que chaque million de mètres cubes importé correspond à une dépense de 300 à 400 millions de francs qu’ il nous faut solder par des envois d'or ou de devises étrangères, dont l'achat contribue à déprécier notre monnaie, à appauvrir la France. C'est plus d'un milliard que nous allons avoir à payer annuellement, de ce fait, aux autres pays d'Europe et d'Amérique.

II n'est pas dans notre pensée de prétendre que nous pourrions nous passer de ces importations et trouver, du moins prochainement, dans nos Colonies de la Côte d'Ivoire, du Gabon et de la Guyane notamment, toutes les quantités de bois dont nous avons besoin. Aussi riches, au point de vue sylvestre, que puissent être ces Colonies, ce n'est pas du jour au lendemain que l'on peut y organiser une production annuelle de plusieurs millions de tonnes de bois et surtout, les bois pouvant y être obtenus étant très différents de ceux que nous utilisons actuellement, qu'on peut les imposer au public en remplacement de ceux qüil a pris l'habitude d'employer. Mais ñimporterions-nous de nos Colonies, pour commencer, que 300.000 ou 200.000 , voire même 100.000 mètres cubes par an, que ce serait toujours autant à ne pas payer à l'étranger; enfin, ce serait l'amorce d'une importation coloniale plus importante. A tous points de vue, ce serait donc extrêmement intéressant.

L'Angleterre, l'Italie, la Belgique. ne sont pas, en ce qui concerne leur production forestière, plus favorisées que la France. Elles aussi doivent importer des bois de l'étranger; elles n'ont pas, comme nous, la ressource de se tourner vers leurs Colonies pour y chercher ce qui leur manque (1). Mais n'envisageons pas les débouchés que nos bois coloniaux pourraient trouver danś ces pays : ceux que leur offre la Métropole leur suffit et leur suffira pour longtemps.

La question est d'importance. Dès 1916 , 1917, en pleine période d’hostilités, le Gouvernement Français, envisageant les difficultés qu il y aurait pour notre pays, après la guerre, à se procurer les stocks considérables de bois qui lui seraient nécessaires, décidait de faire inventorier les ressources de nos forêts coloniales. La mission Bertin partait étudier les peuplements sylvestres de la Côte d'lvoire, du Gabon, puis du Cameroun, déjà enlevé aux Allemands.

(On connaît les résultats de cette mission et les volumineux ouvrages qui ont été publiés à son retour, ouvrages auxquels nous ne saurions trop prier nos lecteurs de se référer.)

Avant la mission Bertin, celle qui avait été confiée au professeur Aug. Chevalier avait permis de déterminer les espèces sylvestres africaines et leur classement scientifique, travail intéressant autant qu'indispensable pour l'établissement d'un "état civil " des bois et que devait compléter, au point de vue pratique, la mission Bertin.

"Puis, en 1918 (2), sous l'impulsion éclairée de M. l'inspecteur général des Trávaux publics Boutieville, devenu, malgré ses nombreux autres devoirs, le plus précieux défenseur et l'apôtre le plus convaincu du bois colonial, le Ministère des Colonies se met résolument à l'ouvre, organise une active propagande et prépare un important effort d'ensemble. La conception générale est la suivante : consituer un vaste consortium où entreraient les administra-

(1) La Vigéria pourrait en fournir pas mal cependant. Quant au Congo Belge, qui possède une zone de forèt très étendue, il ne peut guère en exporter en raison des difficultés et du coût dez transports jusqu'à la côte.

(2) Rapport Weber à la Section des Bois et Produits végétaux coloniaux du Conseil supérieur des Colonies, juillet 1921 . 
tions publiques et privées, grandes consommatrices de bois; susciter, grâce aux besoins de cet organisme, la formation de puissantes entreprises forestières coloniales; aider celles-ci par des avances directes et des commandes dans lesquelles l'Etat jouerait le rôle d'intermédiaire financier.
Chambre sans modification. est ajourné par la Commission sénatoriale. Celle-ci estime qu'il n'y a pas lieu. quelque intéressant que soit le résultat cherché, de créer un nouveau compte spécial. Elle fait observer que le Ministère des Régions libérées possède d'amples crédits de matériaux et elle indique que ce doit être à ce

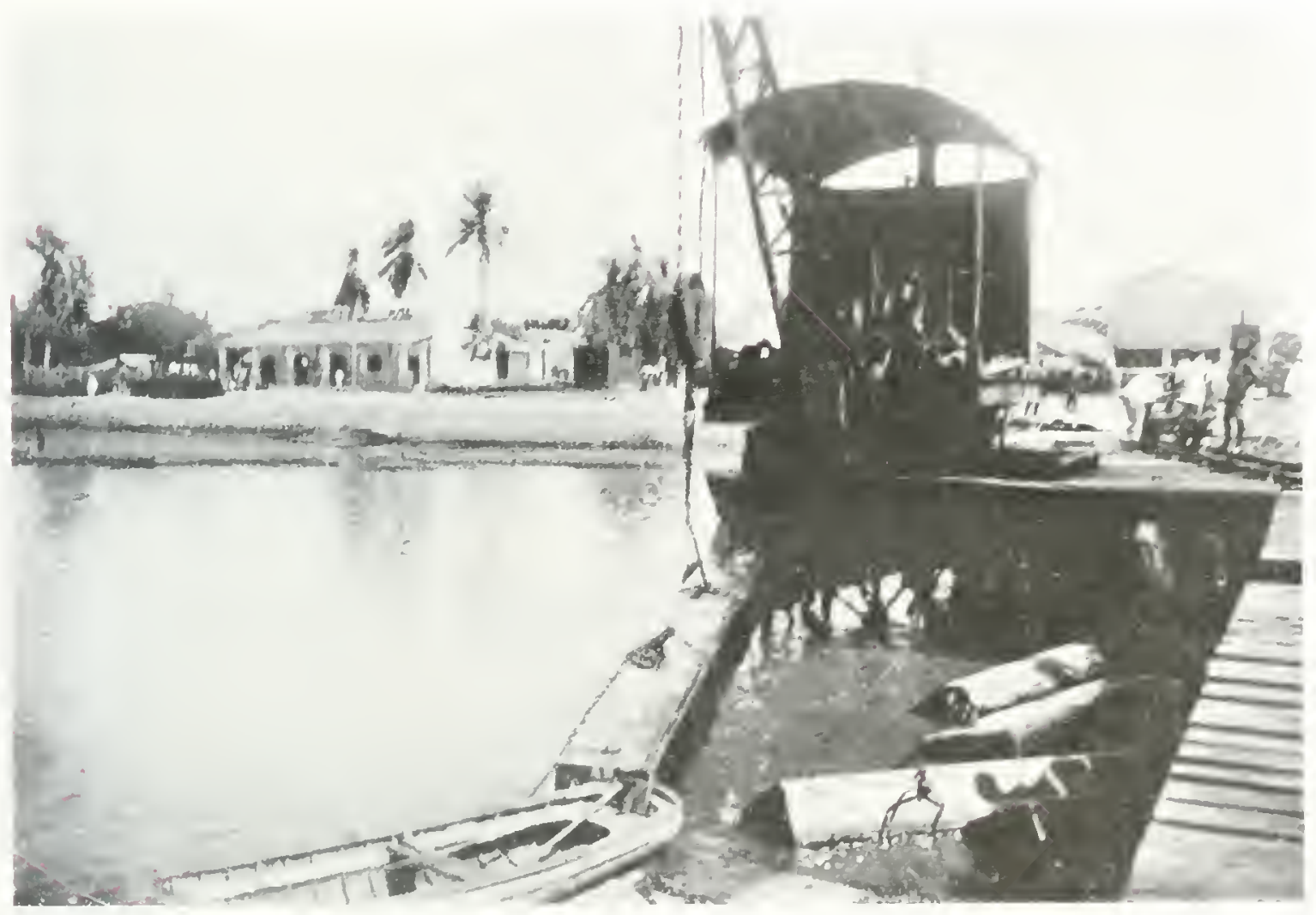

Grue de la lagune du wharf.

Cliche Netayer. - Grand.Barsam

Un projet de loi est déposé en vue d'autoriser le Ministère à constituer, par achats directs aux exploitants, sur la Côte, des stocks de bois bruts ou débités, à en assurer, aux frais du budget, le transport en France, enfin à consentir, aux entreprises forestières et industrielles concourant aux fournitures, des avances remboursables par les livraisons et pouvant s'élever aux quatre cinquièmes du capital ımmobilisé dans l'outillage et les installations.

"Mais ce projet, qui assurait l'avenir des bois coloniaux, après avoir été voté à la
Département de faire les commandes de bois coloniaux utiles à l'ourre de reconstitution.

L'Inspection générale des bois se saisit de la question. Elle passe divers marchés avec la préoccupation exclusive d'obtenir des prix très bas. 11 n'est plus question d'avance. mais l'Etat consent toujours à prendre les bois sur plage et à en assurer le transport et l'écoulement. On commei l'erreur grave, avant qu'aucune scierie n'ait encore été montée pour assurer ces fournitures, de commander 60.000 mètres cubes de bois débitćs. à liver en Afrique. aux prix dèri- 
soires de 60 à 70 francs le mètre cube selon qualités. On néglige de prendre corrélativement des assurances pour le transport. ")

On sait ce qu'il advint de ces marchés. La hausse des prix les rend inexécutables; ils sont résiliés. Les quelques milliers de tonnes obtenus sont revendus sur place ou, s'ils sont importés en France, se détériorent sur les quais du Havre en attendant une destination.

"De nouveau ( 1 ), on retombe dans les incertitudes et les recherches théoriques.

"Pourtant, fonctionne au Ministère des Colonies, sous la présidence du sénateur Barbier, une

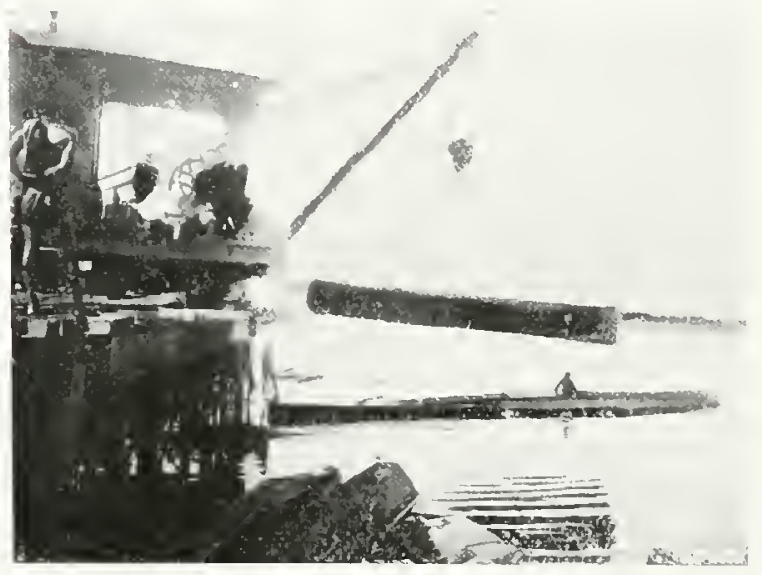

Wharf-Lagune. - Enlevement d'une bille. Clıché Métayer. - Grand-Bassam'

Commission d'essais des Bois coloniaux; elle accomplit le plus utile travail en procédant à l'étude progressive et méthodique des bois coloniaux en vue de leur assimilation aux espèces usitées en France. Mais le président meurt et la Commission ne se réunit plus. Elle ne peut même plus donner la publicité utile aux très intéressantes conclusions auxquelles elle est déjà parvenue.

"Au budget de 1921, dernier vestige des grandes conceptions d'antan, un crédit de 10 millions est cependant maintenu en faveur des bois coloniaux. "

(I) Rapport Weber déjà cité.
Ce crédit de 10 millions ne restera pas inemployé. Le Gouvernement a compris la nécessité de sortir, sans plus tarder, de la période de tâtonnements et d'entrer résolument dans la voie des réalisations. La section du Conseil supérieur des Colonies qui, en juillet 1921, avait à étudier la question de l'utilisation des bois coloniaux, a trouvé en Albert Sarraut, .Ministre des Colonies, une oreille attentive, le concours le plus utile gu' elle pouvaii escompter. Grâce à son intervention auprès de son collègue des Régions libérées, M. Loucheur, le crédit inscrit au budget de 1921 a été utilisé peu après pour l'achat de bois en grumes à un consortium d'exploitants gabonnais. Les prix d'achat ont été calculés de façon que le prix de revient des sciages correspondra à peu près aux prix courants des bois similaires employés jusqu'à ce jour : chêne, hêtre, sapin, etc. Le Comptoir central d'achat, qui est institué auprès du ministère des Régions libérées, dispose des installations et du matériel nécessaires pour débiter cette première fourniture. Les bois sciés seront cédés, après séchage (il faut du moins supposer qu'ils ne le seront pas avant) aux industriels autorisés officiellement à les employer pour la reconstruction des immeubles et usines détruits par les bombardements.

C'est là un premier et intéressant résuliat en faveur de la diffusion des bois coloniaux. Mais il ne faut pas s'endormir sur ce premier et minime succès; il importe, après le Gabon, de penser à la Côte d'lvoire et d'organiser, avec ou sans le concours de l'Etat, l'importation des bois communs de cette Colonie. Cette importation est possible, les essences exploitables commercialement à la Côte d'lvoire, pour être un peu moins variées que celles du Gabon, sont aussi belles et aussi bonnes. Les conditions de leur évacuation et de leur embarquement ne sont pas non plus tout à fait aussi favorables qu'en Afrique équatoriale française; mais la main-d'oeuvre est plus facilement obtenue à la Côte d'lvoire qu' au Gabon et les frais de transport des bois, de la Colonie en France, sont plus 


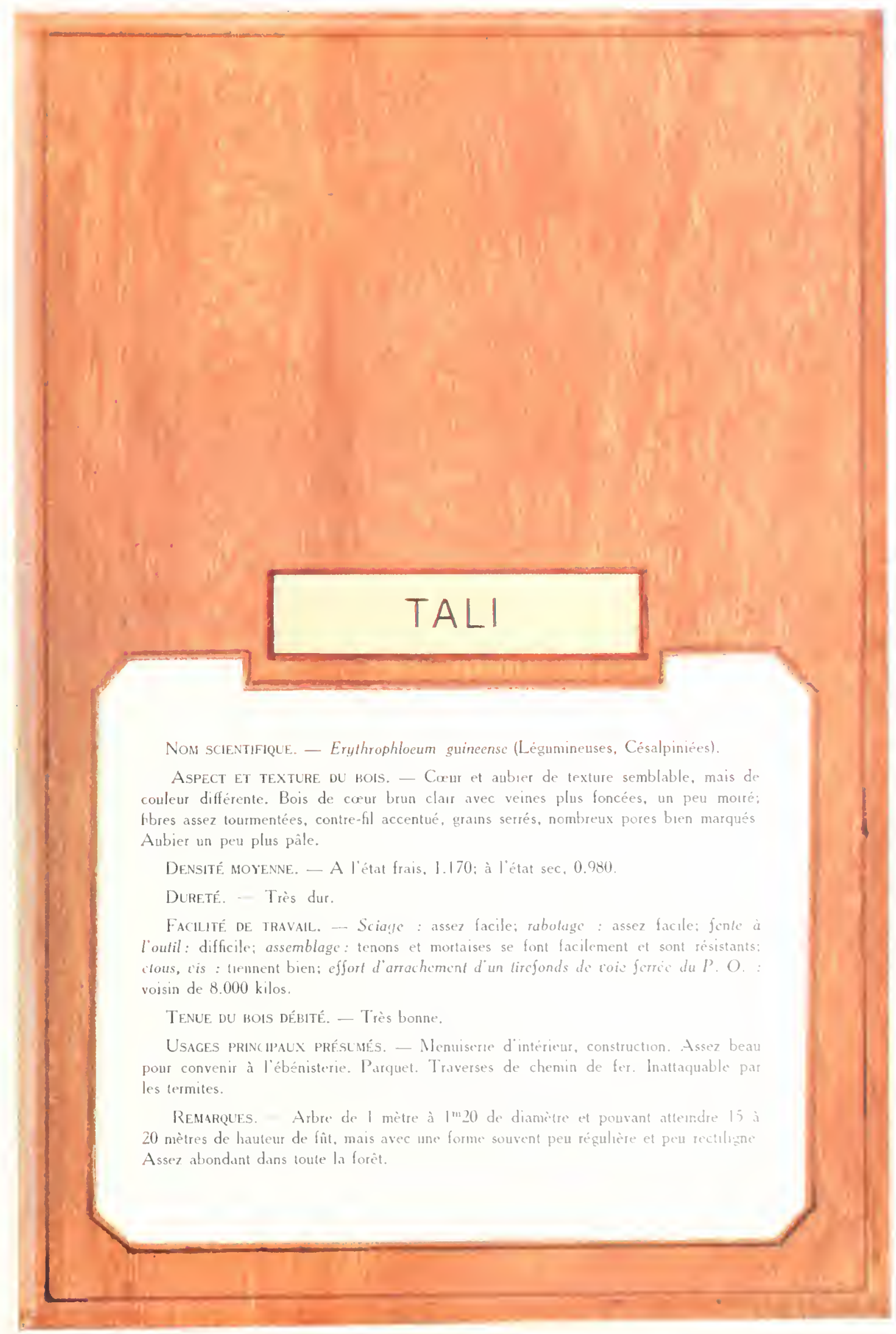



réduits 11 ne s'agit, du reste, que d'établir si les bois de la Côte d'lvoire peuvent être importés et écoulés à des prix assez bas pour concurrencer les bois similaires étrangers; les bois de cette origine ne porteront pas préjudice à ceux du Gabon pas plus que ceux de cette dermère Colonie ne risquent de nuire à la diffusion des bois de la Côte d'Ivoire. Les quantités pouvant
Il faudrait préalablement les peupler et les outiller. Mais demandons-leur, dès maintenant, ce qu'elles peuvent nous donner et prenons des dispositions pour l'utiliser au mieux de nos intérêts. Si par elles notre pays arrivait, peu à peu, à se libérer des importations de bois étrangers, le résultat serait déjà considérable. Nous n’en sommes, hélas, pas encore là !

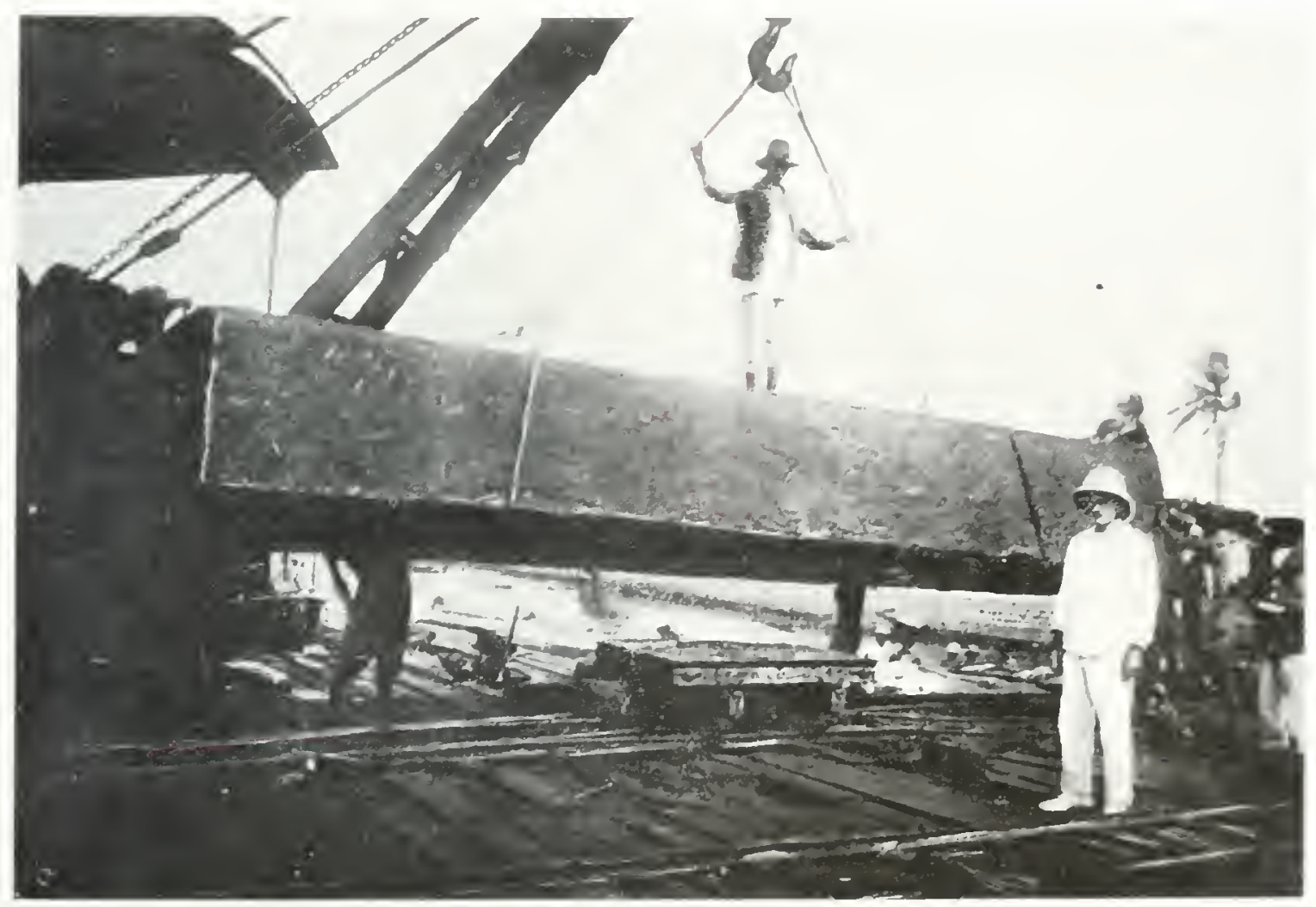

Sur le wharf.

Clichè Métayer. - Grand.Bassam

être importées de ces deux possessions ne constitueront en effet, pendant bien des années encore, qu'une fraction bien minime de ce que notre pays doit acheter au dehors.

Quand on parle des milliards de mètres cubes de bois que contiennent nos forêts coloniales et des centaines de millions de mètres cubes qu'elles pourraient fournir annuellement à l'industrie, on n'est pas conscient de l'importance de ces chiffres. Nos Colonies sont loin, malgré leurs richesses incontestables en bois, de pouvoir fournir des quantités aussi extraordinaires.

\section{Valeur des bois colonialy EN GÉNÉRAL}

"Les bois coloniaux, sont-ils susceptibles de remplacer ceux que la production métropolitaine ne suffit pas à nous fournir et que nous devons importer d'Europe? $C^{\prime}$ est la première question qui se pose, le premier doute qu "il faut s"attacher à dissiper.

"On est habitué, en effet, à employer. pour les divers usages, des bois déterminés, dont une expérience séculaire a appris à connaitre et 
apprécier les qualités propres. Pour établir la charpente des bâtiments, on a recours, par exemple, au sapin ou aux résineux qui s'en rapprochent, à la fois légers et résistants; ce sont les mêmes essences qu'on emploie couramment dans la menuiserie commune et la caisserie, en concurrence avec le peuplier. Dans le charronnage, la carrosserie, l'orme et le frêne trouvent des applications dans lesquelles on ne saurait les remplacer. C'est surtout en ce qui concerne les résineux, sapin ou analogues, que la disproportion entre la production nationale et la consommation est la plus marquée; aussi les fait-on venir chaque jour de l'étranger en plus grandes quantités. Or, on s'imagine les bois coloniaux tout difféents de leurs similaires originaires d'Europe; les "Bois des Iles ", ainsi qu'on les appelle dans le commerce, sont surtout, pense-t-on, des bois lourds, à couleurs vives, tels l'acajou, le palissandre et l'ébène.

"Il est certain que, pendant longtemps, on n'a guère eu avantage à exporter des Colonies que des bois de luxe, vendus assez cher pour payer largement les frais d'exploitation et de transport. Mais, loin de composer toute la forêt coloniale, ils ne constituent qu'une des nombreuses variétés de la flore sylvestre tropicale dont la richesse et la diversité ne souffrent d'ailleurs aucune comparaison avec celle des forêts européennes et qui, en outre, diffère, non seulement de continent à continent, mais encore dans l'étendue d'une même région. Il est incontestable que, dans les Colonies voisines des tropiques, les bois de grande densité, à la fibre résistante et dure, prédominent beaucoup plus que dans nos forêts. On ne saurait nier non plus qu' aucune de nos Colonies ne peut fournir des produits du type sapin ou pin, tels que nous les importons en si grandes quantités. Tout au plus I'Indo-Chine, dans ses régions montagneuses, d'un accès difficile, en contient-elle d'importantes réserves. Mais il est cependant un grand nombre d'esences, que leur poids et leur dureté rapprochent plus ou moins du chêne ou du noyer et qui, douées de qualités variées, pourraient se substituer dans l'ameublement, la menuiserie, la fabrication des wagons et des voitures, aux types de bois couramment en usage. D'autres, plus légers, plus tendres, pourraient être utilisés comme le peuplier, et d'autres encore remplaceraient le sapin, pour certains usages, dans la construction et la menuiserie.

"La substitution des bois coloniaux à nos bois européens exige cependant certaines modifications dans les habitudes des artisans. Pour débiter et mettre en ouvre ces essences, pour eux nouvelles, il y aura lieu de recourir à des procédés différents, que la routine du métier sera portée à n'accueillir d'abord qu'avec défaveur. Mais ce ne sera là qu'un obstacle passager que l'on aura peu de peine à surmonter si l'on prend soin de déterminer, au préalable, la technique spéciale qui leur est applicable.$$
\text { ** }
$$

"Les bois européens peuvent donc être remplacés par les bois coloniaux pour la plupart des usages courants.

"Depuis quelques années, le chêne du Japon vient faire concurrence, sur le marché européen, au chêne des futaies françaises et au chêne importé de Hongrie. Il semble bien qu'on soit autorisé à conclure, de ce fait, que toute essence coloniale susceptible de remplacer le chêne pour nos usages peut aussi bien supporter les frais de transport de même importance que ceux qui grèvent le chêne qu'on fait venir du Japon.

"De l'exposé qu'on vient de lire, il est permis de tirer les conclusions suivantes : d'abord que nos Colonies sont pourvues de ressources forestières susceptibles de suppléer à l'insuffisance de nos produits métropolitains; ensuite que rien ne s'oppose, à priori, à ce que les bois qui en proviennent disputent notre marché à ceux qu'il importe d'Europe et d'Amérique (I). "

Rien ne s'y oppose, en effet. Pour tous ceux qui connaissent les bois coloniaux, nous parlons

(1) $\mathrm{D}^{\mathrm{r}}$ Chauveau, sénateur de la Côte-d'Or. La France Agricole et la Guerre. (Ouvrage Bertin, Mission forestière coloniale.) 
en ce moment de la Côte d'lvoire, il ne saurait y avoir de doute que la grande majorité des essences pouvant être exploitées dans cette Colonie, essences dont certaines sont déjà réputées en ébénisterie et en menuiserie fine, ne puissent parfaitement être employées à tous les autres usages industriels. Laissons de côté les essences trop tendres, qui ne pourraient pas concurrencer, au point de vue prix, les peupliers et pins
Tous les essais tentés jusqu'à ce jour n’ont pas été effectués dans les conditions de séchage et avec l'outillage voulus. Il en est résulté une légende sur les difficultés de travail de ces bois. leur dureié, leur éclatement sous le clou, etc.. légende basée sur des renseignement; inexacts et que nous nous attacherons à détruire.

"Les bois coloniaux , dit M. Gillet (1), l'industriel parisien bien connu, qui les étudie pra-

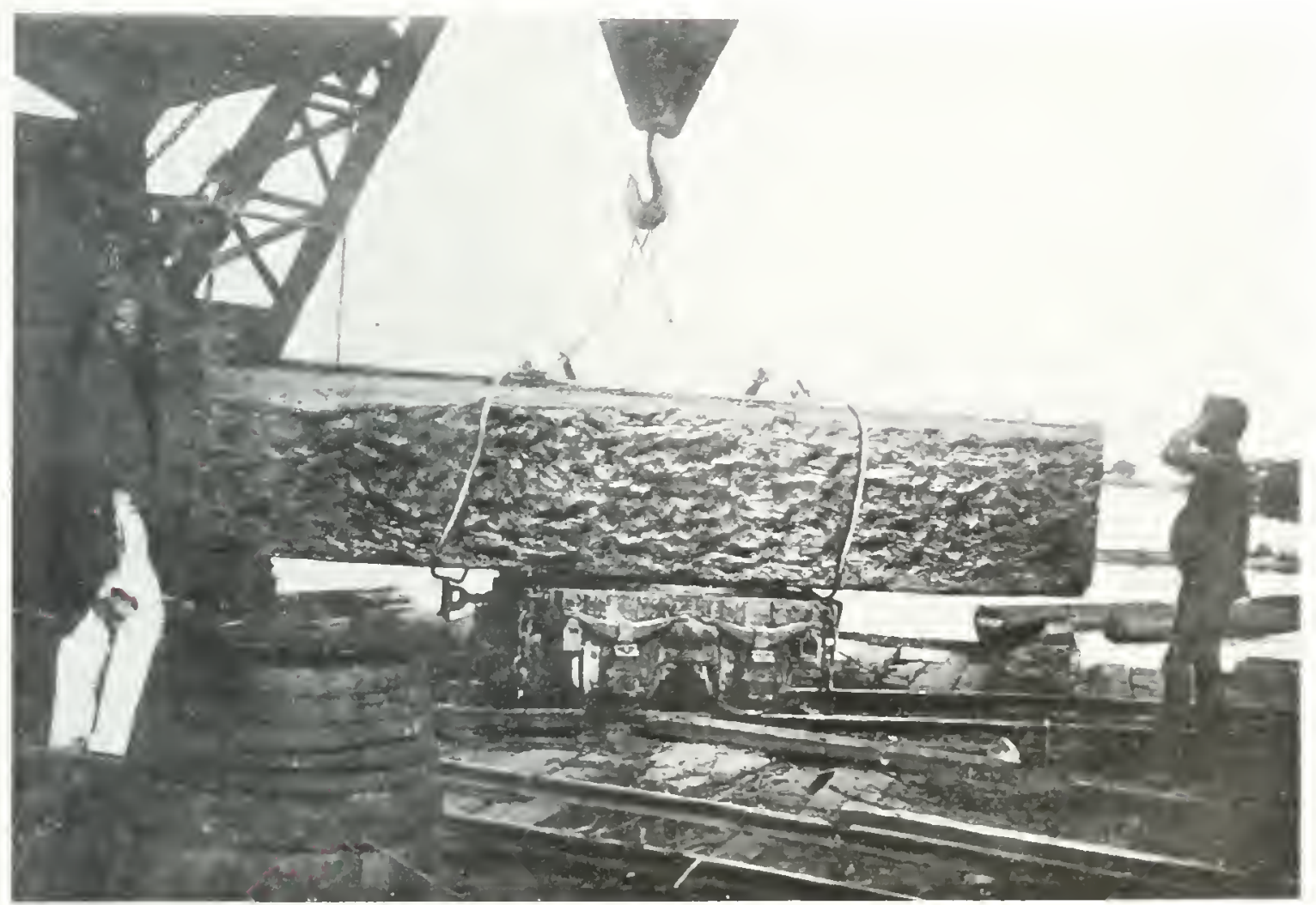

Sur le wharf. - Depót de la bille sur wagon.

Cliche Metayer. - Grand-Bassam

ou sapins vulgaires. Nous en avons beaucoup d'autres de meilleure qualité, qui se travaillent aussi facilement et qui remplaceraient avantageusement les sapins de choix, les pitchpins, les platanes, les hêtres, les chênes pour la menuiserie, la charpente, le parquet, peut-ĉtre le merrain; nous en avons de beaucoup plus dures qui, soit par leur poli, soit par leur imputrescibilité, peuvent trouver leur utilisation dans l'outillage, le pilotis, les constructions navales, la traverse de chemin de fer, etc. tiquement depuis seize ans, se travaillent parfaitement bien, à condition qu' on les connaisse et qu'on prenne, au préalable. quelques précautions élémentaires.

Voici ce que disait, au surplus, M. Gillet. dans une conférence récente à la Société centrale des Architectes (1) :

Le travail du bois conprerd deux genres d'opérations : on commence par le préparer et

(I) Rapport Weber déjà cité 
le façonner aux outils mécaniques, puis le bois est ensuite travaillé manuellement et reçoit, en demier lieu, la façon définitive.

"La première opération comprend le sciage. On a dit que beaucoup de bois coloniaux ne pouvaient se scier. Ils ne se scient pas tous, en effet, avec les mêmes scies; pour certains, on utilise la scie alternative; pour d'autres, la scie circulaire et la scie à ruban. On doit donc procéder par sélection et employer, pour les diverses essences, les scies les mieux appropriées.

"Il existe toutefois certains bois qui présentent des difficultés au sciage.

"On rencontre, par exemple, des bois siliceux qui émoussent rapidement la denture de la scie; après avoir exécuté quelques traits, la scie ne fonctionne plus. Pour résoudre cette difficulté, d'un ordre spécial, il suffisait de poser la question suivante aux fabricants d'acier : "Pouvez-vous nous donner une scie faite avec " un acier spécial, qui puisse résister au sciage " des bois siliceux? " Les fabricants d'acier ont étudié la question et nous devons signaler qu'elle est actuellement résolue. Tout dernièrement, nous avons mis en service une scie qui a travaillé des bois siliceux pendant deux heures sans affutage. On peut donc dire que le but est atteint.

"Ensuite, nous passons au corroyage et au rabotage du bois qui s'exécutent dans les mêmes conditions que pour les bois métropolitains; certains bois ont un fil et un contre-fil, mais, en prenant les précautions élémentaires de réglage des couteaux, ces bois se travaillent aussi bien que les autres, et sans arrachement.

"Les assemblages, tenons et mortaises ne présentent pas de difficultés; pour exécuter les tenons dans les bois tendres, nous conseillons l'emploi de "tencheuse " à porte-outils hélicoïdaux travaillant à travers fil.

" Le bouvetage et la mouluration sont exécutés avec les outils habituels tels que toupie, " parqueteuse ") et "moulurière ") nous conseillons, pour fcertains bois siliceux, l'emploi d'acier rapide pour la confection des fers à moulures; ils donnent d'excellents résultats.

" Le râclage et le ponçage mécaniques des bois sont tout à fait recommandables pour le façonnage des bois coloniaux, principalement pour ceux à fil et contre-fil, auxquels ils donnent leur aspect définitif.

"En somme, le travail mécanique peut s'exécuter dans d'excellentes conditions. Certains bois, qui sont très durs, ont besoin d'outils spéciaux et nous recommandons à tous les industriels qui auront à les travailler, de poser le problème à leurs fabricants d'acier. Je suis convaincu qu'ils arriveront très facilement à résoudre cette question.

" II nous reste maintenant à déterminer les conditions du travail manuel des bois coloniaux.

"Il n'est pas besoin de dire que les ébénistes, depuis longtemps, travaillent très bien les bois exotiques; c'est grâce à leur talent et uniquement avec les bois coloniaux qu'ils confectionnent les plus beaux meubles que nous exportons dans le monde entier.

"En introduisant les bois coloniaux dans l'industrie nous rencontrons parfois la résistance de l'ouvrier. Celui-ci, plus ou moins routinier, habitué au sapin et au chêne, n'apporte pas toujours toute la bonne volonté nécessaire pour travailler ces bois.

"Il faudra probablement que l'ouvrier moderne apporte certaines petites modifications dans ses méthodes, mais nous pouvons affirmer qu'un menuisier ou ébéniste consciencieux, qui consentira à faire abstraction de la routine, arrivera, au bout de quinze jours, à travailler très facilement les bois coloniaux. Il suffit simplement, pour le rabotage, de régler le fer et le contre-fer de l'outil de telle façon que le travail à exécuter sur les bois à fils entrecroisés se présente dans des conditions favorables (I).

"Nous sommes convaincus, par l'expérience,

(1) On fabrique du reste, pour les bois à contre-fil, des rabots spéciaux. 


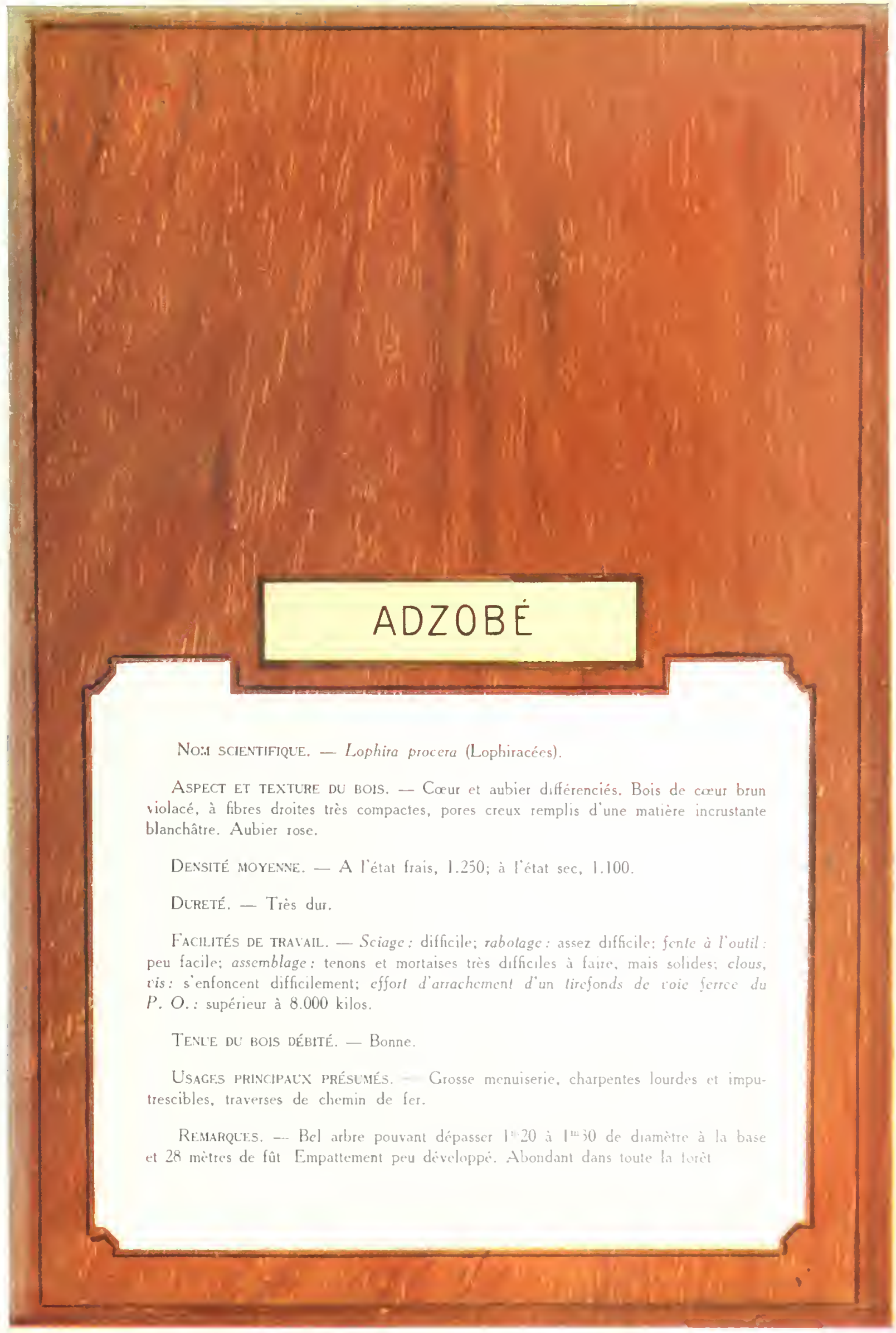



qu'au bout de peu de temps cet ouvrier ne fera plus aucune distinction entre le travail des bois métropolitains et celui des bois coloniaux; il sera même intéressé par la beauté des bois qu il aura ainsi utilisés et il éprouvera une satisfaction personnelle à avoir vaincu une difficulté passagère. ")

M. Gillet ne se contente pas de faire de la parfaitement bien, malgré l'humidité et la chaleur, pourvu qu'elles soient à l'abri du soleil et des intempéries. Les essences les plus dures peuvent être employées indifféremment pour les travaux d'intérieur comme pour ceux d'extérieur. Elles résistent à tout, même aux termites, qui sont la plaie des bois de construction en Afrique occidentale.

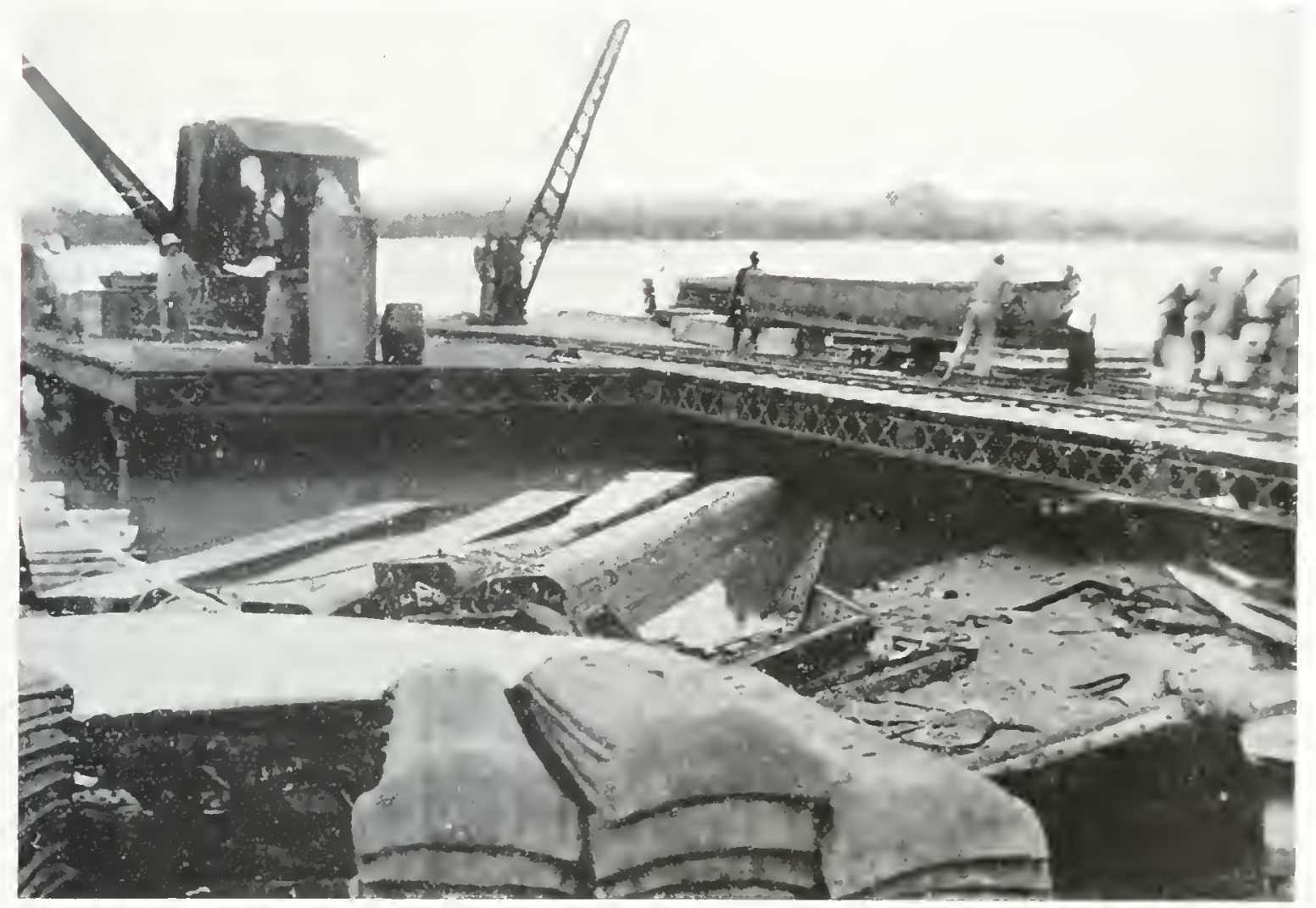

Les billes sont montèes sur l'appontement de la lagune, placees sur wagonnel pour etre ensurte, soit embarquees, soit deposees sur le terre-plein.

Cliche Asence economique A. O.F.

propagande pour les bois coloniaux, il les utilise dans ses usines, construit notamment des pavillons en bois pour habitations "qui joignent, dit M. Weber, à tous les avantages de la solidité et de la sécurité la plus parfaite, celui de l'élégance française. "

Signalons qu'à la Côte d'Ivoire même toutes les constructions sont actuellement faites en bois du pays et que, même les essences les plus tendres, comme le Bahia, se comportent
Les essais de durée, de conservation, de résistance, de sciage, effectués en France, n'ont pas été moins satisfaisants (1). Les plus intéressants et les plus concluants ont sans doute été faits par les Compagnies de chemins de fer français. On sait, en effet, qu'un Consortium a été créé dans le but unique de se procurer, dans nos Colonies. les bois nécessaires aux grandes Com-

(1) Voir ourrage Bertin. Mission forcstière coloniale, pages 608 et 681 . 
pagnies pour la construction, le wagon et la traverse de chemin de fer. Ce Consortium a monté, cn 1920, au Gabon, une importante exploitation forestière; il achète, en outre, des lots de bois d'essences déterminées. Il avait tout d'abord jeté ses vues sur la Côte d'Ivoire; les pourparlers engagés à ce sujet avec l'Administration de la Colonie n'ont échoué que pour des raisons absolument étrangères à la concession forestière sollicitée et à la qualité des peuplements.

De l'avis unanime de tous les industriels et architectes qui les ont examinés d'un peu près, les bois africains sont absolument "aptes, en les employant chacun selon ses propriétés, à tous les usages de la construction, de la charpente et de la menuiserie. M. Leduc, architecte diplômé de la Ville de Paris, les déclare particulièrement propres à l'édification des maisons en pans de bois dans lesquelles il voit le moyen d'activer la reconstruction des Régions libérées et de résoudre la crise du logement par une impulsion nouvelle à donner à l'habitation à bon marché.

"Bref, on peut l'affirmer, qu 'il s'agisse de poutres, de lames de parquets, de panneaux, de moulures, de châssis de fenêtres, de persiennes, de marches d'escalier, de moyeux de rouesł de carrosserie, de wagons, d'aménagements intérieurs de navires, etc., etc., les bois d'Afrique peuvent convenir à tout (I). "

Nous allons passer rapidement en revue les bois de la Côte d'lvoire dont nous préconisons l'importation pour la plupart de ces usages.

\section{Essences a exploiter a la Cote d'IVOIRE ET A IMPORTER EN FranCE.}

Ainsi que nous l'avons déjà déclaré, nous nous garderons bien de préconiser l'exploitation et l'importation en France de tous les bois re-

(1) Rapport W'eber déjà cité. connus intéressants à la Colonie. Nous nous limiterons aux meilleurs parmi ceux qui peuvent être coupés, en grande ou moyenne quantité, dans une zone de 10 kilomètres de profondeur, au maximum, bordant la voie ferrée, les lagunes et les rivières $y$ accédant qui sont navigables ou flottables la majeure partie de l'année. De cette façon, nous réduirons le plus possible nos frais d'évacuation et le prix de revient de nos bois; nous réduirons également le nombre des essences et rendrons, de ce fait, leur écoulement plus facile.

Quatre essences dominent nettement dans toute cette zone : le Bahia, dans les terrainj bas et marécageux qui bordent rivières et lagunes: le Rikio, immédiatement à côté ou derrière, sur les terrains plus fermes; puis, plus en arrière, le Dabéma et l'Avodiré.

Ces quatre essences doivent représenter, à elles seules, une moyenne de $25,300 / 0$ peutêtre, de l'ensemble des arbres exploitables de la zone en question. Toutes les quatre sont excellentes. A côté d'elles il en est d'autres, dont quelques-unes de meilleure qualité encore, mais beaucoup moins abondantes, qui sont également à exploiter. Ce sont, avec quelques Acajous et Acajous-Tiama, des bois d'ébénisterie, comme l'Iroko, très commun à certains endroits, dans la région de Grand-Lahou notamment, le Makoré, le Badi, le Bossé, l'Assas, l'Oboto, l'Olon (1), tous asse? rares dans la partie de forêt qui nous occupe, mais dont il y a néanmoins lieu de tenir compte; puis des bois de menuiserie et de charpente comme le Niangon, le Sénan, le Framiré; des bois plus lourds, comme le Sougué, l'Adjouaba, le Tali, le $F_{\text {ou }}$, susceptibles d'emplois divers; enfin des bois à traverses ou pilotis comme le Bodoia, le Kroma et l'Adzobé.

Au total, cet ensemble doit représenter,

(1) Nous ne considérons pas, en raison de sa densité et de sa dureté, l'Apomé comme un bois d'ébénisterie. malgré qu'il ait été classé dans cette catégorie par la Commission des Bois coloniaux. 
pour la zone de 10 kilomètres de profondeur que nous indiquons, 50 à $550 / 0$ du cube total de la forêt, dont $350 / 0$ de bois, densité infèrieure à 1.000 , pouvant être "flottés " à l'état vert ou après séchage de quelques mois, et 15 à $200 / 0$ de bois lourds non flottables.

Il est bien entendu qu'en dehors des essences d'ébénisterie dont on aura toujours le placement l'attention de nos lecteurs et qui, dans son ensemble, est un peu moins dense que la zone située en arrière, pourrait fournir un tonnage de bois déjà considérable. Son développement doit atteindre au moins 375 kilomètres. Sa superficie serait donc, au minimum, de 375.000 hectares. $\mathrm{Si}$ l'on prend, comme cube total moyen, 150 stères (1) seulement de bois d'ourre à

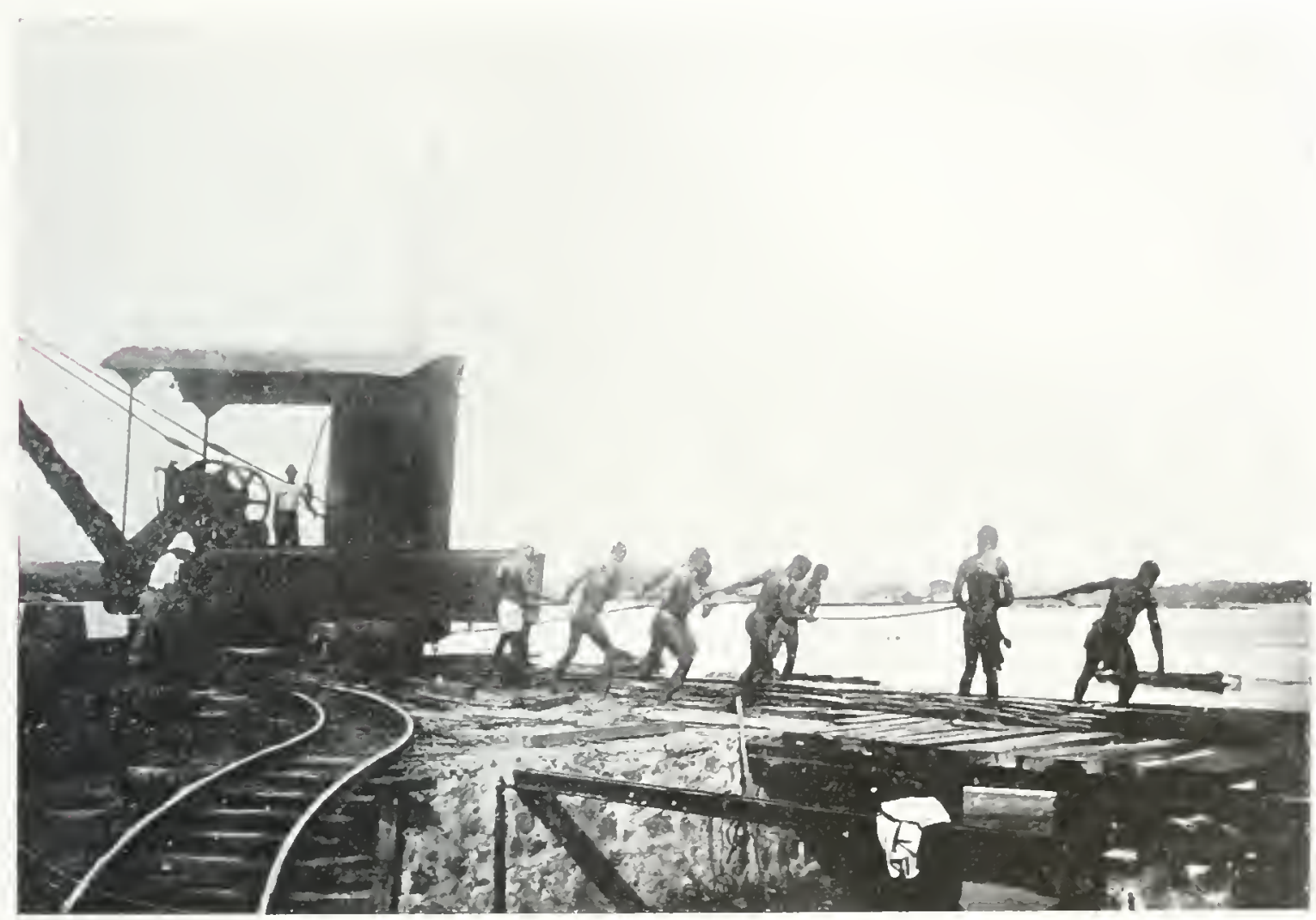

Tirage d'une bille sur le wharf.

Cliché Métayer. - Grand.Bassam

à des prix rémunérateurs, et des quatre essences dominantes que nous avons énumérées, les autres essences, quelles que soient leurs qualités, ne devront être exploitées que si l'on est susceptible d'en réunir, à l'endroit où l'on exploite, un tonnage intéressant. Il serait inutile, en effet, de se donner du mal pour lancer un bois dont on ne pourrait pas assurer régulièrement, par la suite, des livraisons importantes.

En s'en tenant aux seules essences principales, la partie de forêt que nous signalons à l'hectare, dont 500,0 en essences utilisables immédiatement, on obtient un chiffre de :

$$
\frac{375.000 \cdot 150 \times 50}{100}=28.125 .000 \text { stères }
$$

ou mètres cubes pouvant, répartis sur 80 années (temps maximum qui parait nécessaire pour le repeuplement, en arbres adultes. des parties

(1) Pour tenir compte des saranes cottières des espaces cultivés ou couverts de forèt secondaire. 
de forêt exploitées) donner lieu à des coupes annuelles de 350.000 mètres cubes. Largement de quoi alimenter une ou plusieurs industries importantes.

Au-dessous des dessins en couleur représentant les différentes essences dont nous préconisons l'exploitation et l'importation, nous avons indiqué les caractéristiques principales des bois et les résultats des esais auxquels ils ont donné lieu de différents côtés, notamment par les missions Bertin et Salesses, ce dernier opérant pour le compte des grandes Compagnies de Chemins de fer français. Nous allons ajouter, à ces indications d'ordre général, quelques précisions concernant les différentes essences que nous recommandons particulièrement :

\section{I" Bois d'ébénisterie (Exploitation accessoire)}

Makoré, Badi, Bossé, Asas. Oboto, Olon (1).

Makoré et Bossé sont déjà coupés en petites quantités par les exploitants. Le Badi également, mais on le rencontre moins fréquemment. Le Makoré fournit un beau bois rouge semblant convenir particulièrement pour le meuble en bois plein; il en est de nombreuses billes qui sont moirées. On lui reproche de se fendre au séchage, c'est un inconvénient dont il est possible de limiter les effets en mettant le bois sécher à l'ombre et à l'abri des vents trop secs. Il serait peut-être encore mieux de débiter immédiatement les billes en plateaux et de les mettre à sécher sous hangar fermé.

Le Bossé (2) est moins fin, quoiqu'il en est de jolies billes très ramagées qui feraient également du beau meuble ou de la belle menuiserie

(I) Nous nous sommes déjà occupés du Tiama et de l'Iroko, titre II, pages 58,61,62, 63.

(2) Le peintre Dupuis a reproduit très fidèlement les échantillons de bois qui ont été mis à sa disposition. Certains de ces échantillons que nous nous sommes procurés au Jardin colonial à Nogent laissaient toutefois à désirer; les planches en couleur ne donnent donc pas toutes une idée très exacte des bois qu'elles représentent : c'est le cas notamment pour le Bossé et le Framiré dont les dessins représentent des bois unis et de fil, alors que ces essences d'intérieur. Blanc crème au débitage, le Bossé rosit rapidement au séchage. Il prend très bien le chromate de potasse. Le Sipo, faux-acajou, dont nous avons parlé au Titre $\mathrm{I}^{\mathrm{r} r}$, semble être un Bossé de belle qualité.

Le Badi est un excellent bois sous tous les rapports. On ne peut lui reprocher que d'être un peu lourd et dur à travailler. La plupart des billes présentent des effets moirés. C'est un bois qui convient également pour le meuble massif.

Asas, Oboto et Olon sont beaucoup moins connus et ne donnent encore lieu à aucune exploitation. Ce sont trois jolis bois qui trouveraient certainement leur emploi dans les industries d'ameublemeni.

Ces trois essences, sauf à de rares endroits de la forêt, sont trop peu abondantes pour que les exploitants actuels essaient d'en tirer parti commercialement. Nous ne les mentionnons pas qu'à titre documentaire, cependant, car il est possible que, par la suite, on en découvre des peuplements plus importants qui justifieront leur exploitation.

L'Oboto est peut-être d'emploi moins facile, mais l'Asas et l'Olon sont deux beaux bois satinés qui trouveraient amateurs s'ils étaient obtenus couramment. L'Olon est toutefois un peu lourd; cela ne lui enlève rien de sa qualité, au contraire.

Les six essences ci-dessus ne sont donc pas susceptibles de donner lieu à un mouvement d'exportation important. Il est difficile, à chaque exploitant, d'en réunir un tonnage appréciable et la vente en France par trop petits lots ne peut, d'autre part, donner de bons résultats. Les coupeurs d'acajou qui, pourtant, ne devraient pas les négliger, auraient intérêt à grouper leur

sont généralement assez ramagées; le Rikio, dont le bois est plus ou moins marbré; enfin le $F_{\text {out }}$, bois d'un beau rouge uni et très homogène, alors que le dessin représente un échantillon pris sans doute dans une branche et qui donne deux tons différents.

Les densités que nous avons indiquées sur les fiches d'essai, sont des densités movernes. Elles sont, en effet. très variables pour une même essence, suivant la région ou la nature du sol. Dans les terrains humides ou marécageux, la densité des bois est généralement plus légère que dans les terrains sains ou rocailleux. 


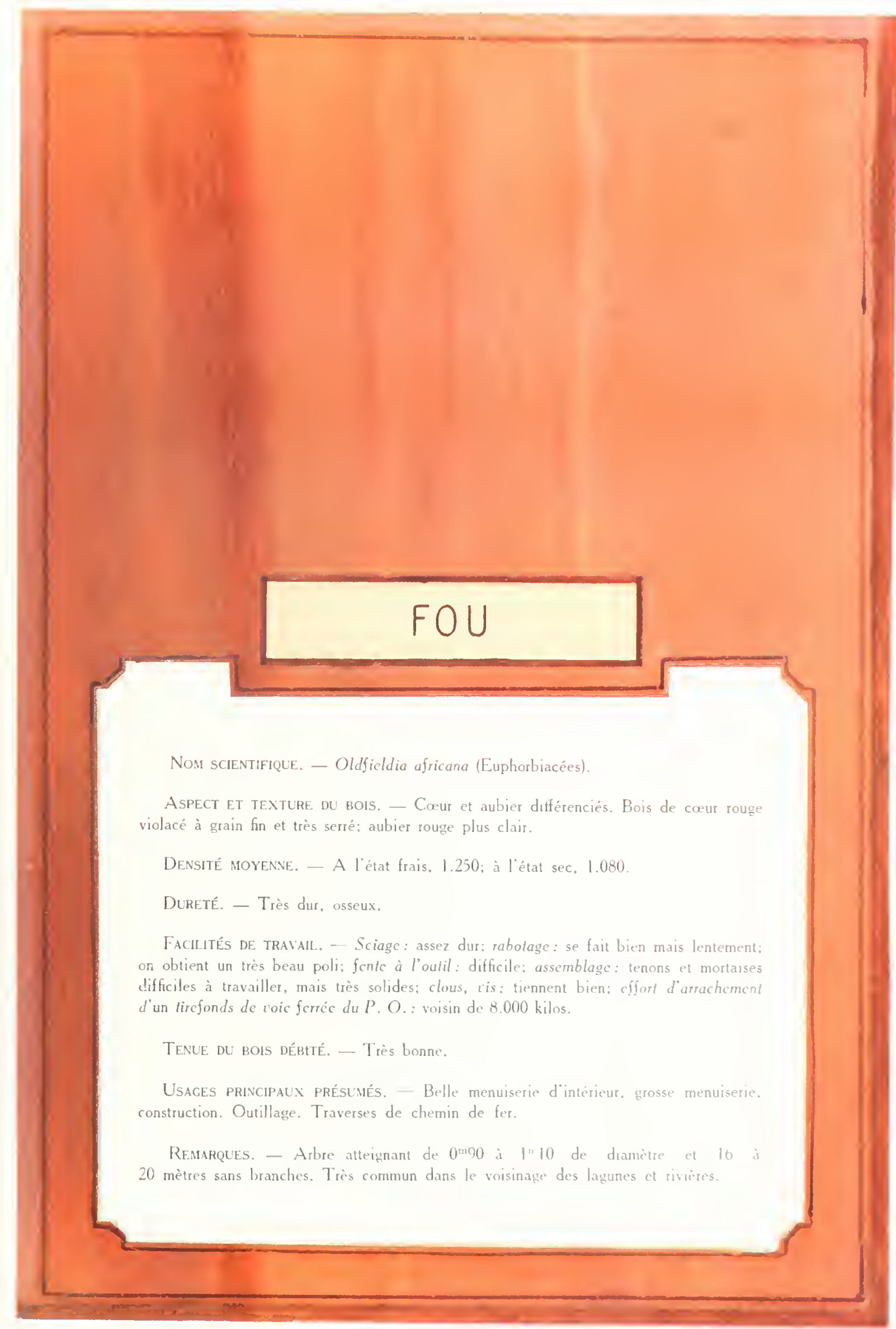


production et à la faire vendre, sinon en bloc, du moins par lots assez importants. lls obtiendraient, en agissant ainsi, des prix certainement supérieurs à ceux qui leur sont offerts actuellement quand ils essaient de vendre chacun de leur côté. Une Société qui entreprendrait en grand l'exploitation des bois de menuiserie et charpente et leur importation en France, pourrait acheter aux autres exploitants ces bois divers d'ébénisterie et les écouler avec sa propre production.

$2^{\circ}$ Bois de menuiserie et de charpente de densité inférieure à 1.000 , exploitables en grosses quantités. Exploitation principale: Avodiré et. Bahia, Dabéma et Rikio.

Deux bois assez tendres et deux bois durs. Ces quatre essences sont, comme nous l'avons exposé, les plus abondantes de la région côtière. Le Bahia, notamment, peut s'obtenir en grosses quantités et à très bon compte; c'est un bois gris-jaune, de bonne qualité, qui peut remplacer pin et sapin de choix pour le meuble à bon marché et tous travaux de menuserie et de charpente légère (1).

L'Avodiré est peut-être supérieur, comme qualité, au Bahia. Il convient pour tous travaux de menuiserie, de moulure et de charpente légère.

Le Dabéma et le Rikio sont plus lourds et plus durs. Ils peuvent remplacer chêne et hêtre, platane, etc... Le Dabéma surtout, est un très beau bois satiné, qui se travaille bien et se rapproche, comme ensemble, des beaux chênes de France; les pores sont un peu plus creux, mais le ton est plus doré, plus foncé. Il est plus beau à notre avis. On pourrait l'employer pour du meuble, mais c'est pour la lame de parquet qu'il semble le plus indiqué. Il est à essayer également pour la fabrication de futailles; il ne paraît pas toutefois suffisamment maillé pour cet usage et il est de fente difficile.

(1) On a signalé que certaines billes de Bahia étaient inégalement dures, après séchage. C'est un fait à vérifier. Pour les emplois en charpente, I"inconvénient ne serait pas bien grand.
Le Rikio semble être d'une qualité plus commune. C'est plutôt un bois de construction qui peut remplacer chêne et hêtre. Sa maille est assez grande, sa fente facile; il pourrait donc être débité en merrains et il est à étudier à ce point de vue.

D'assez nombreux Rikios sont creux à l'intérieur. Des déchets importants au débitage seraient donc à redouter si l'on ne prenait des précautions pour réduire au minimum le nombre de billes creuses pouvant être exportées de la Colonie; mais il suffit de tronçonner les arbres suspects en billes assez courtes ( 4 mètres, par exemple) pour éliminer, à peu près complètement, les parties qui sont défectueuses.

Dabéma et Rikio sont, à l'état vert. d'une densité voisine ou légèrement supérieure à 1.000. Pour les faire flotter, il faut done au préalable les faire sécher légèrement, résultat qui sera obtenu d'autant plus rapidement que les billes seront équarries dès abattage. C'est là, du reste, une opération que nous conseillons pour tous les bois dont on voudra faire l'exportation en billes, exception faite de ceux qui peuvent être destinés spécialement au contreplacage et qui doivent être déroulés. Non seulement, en effet, l'équarrissage permet d'obtenir une réduction plus rapide de la densité, il évite aux bois de s'échauffer ou de se piquer du fait de l'humidité, il fait découvrir la plupart des défauts et permet une élimination immédiate de tout ce qui peut laisser à désirer; il réduit très sensiblement les frais de transport par bateaux. les billes s'arrimant mieux et, de plus, étant moins lourdes que des grumes pour une valeur de bois égale.

3" Autres bois de menuiserie et charpente de densité inféricure à 1.000 , exploitables également, mais en quantités moins importantes: Niangon, Senan, Framiré.

Ces trois essences sont excellentes à tous points de rue et peurent remplacer couramment chêne, hêtre, platane et même pitchpin, quoique très différentes, comme contexture, de celles-ci.

Le Niangon est un faux-acajou, il est d'une 
très bonne tenue au débitage. Il est à étudier, ainsi que le Senan, au point de vue utilisation en tonnellerie. Ce dernier, couleur mise à part, est un bois absolument analogue au Rikio. Certains ébénistes qui en ont examiné, à la Foire de Bruxelles, des échantillons tì̀s marbrés, ont laissé entendre qu'ils l'achèteraient volontiers pour faire du meuble plein, copie d'ancien. C'est à voir. Il est certainement utilisable, en touî cas, en menuiserie et en charpente.

Le Framiré est également un très bon bois qui rappelle, comme grain et dessin, le frêne de France. Il est léger et souple. Il est malheureusement peu commun dans la région côtière (1).

Ces trois essences ne devront, du reste, être exploitées que s'il est possible de réunir un tonnage intéressant de chacune d'elles. Elles sont loin d'être uniformément réparties. Il n'y a d'ailleurs, pas intérêt, nous l'avons déjà fait remarquer, à importer en France une trop grande variété de bois car plus la liste en sera longue, plus leur placement sera difficile (2).

$4^{\circ}$ Bois semblasl convenir plus particulièrement pour l'outillags : Fou.

La plupart des essences dures de la Colonie, pourraient être employées avec succès pour l'ou-

(1) A cette liste déjà longue nous aurions pu ajouter: le Fraké, l'A iélé et l'Abalé.

Le Fraké rappelle le chêne de Hongrie. C'est un bois de bonne qualité et de conservation facile. Il trouverait certainement écoulement à un prix rémunérateur: cette essence est toutefois assez rare dans l'ensemble de la forêt; elle est, par suite, difficilement exploitable.

L'Aiélé, ou Okoumé de la Côte d'Ivoire, est par contre assez commun dans la forêt. $\mathrm{C}^{*}$ est un bois très léger qui ressemble beaucoup comme grain à l'A cajou, mais il est à peine rosé. C'est en quelque sorte un Acajou blanc. Il pourrait sans doute être utilisé en menuiserie et pour les industries de contre-placage. C'est toutefois un bois chanvreux et de conservation délicate à la Colonie; nous n'osons pas le recommander; il ne vaut pas du reste, comme qualité, l'Okoumé du Gabon.

L'Abalé, qui est un assez joli bois, se conserve aussi très difficilement. Il se pique dès abatage, se gauchit, éclate, il pourrait cependant être étudié pour le merrain, car il est maillé et de fente facile. On ne peut en tout cas envisager son exploitation que si l'on possède à la Colonie une installation de dessévage, le bois ćtant débité et traité dès abatage.

(2) C'est la raison pour laquelle nous avons cru tillage et remplacer les chênes verts, buis, cormier, gaïak, pour la fabrication de rabots, varlopes, bouvets, etc., ainsi que pour la meule en bois, la roulette de meuble, la navette de métier à tisser, etc. Mais il en est une qui, par son grain fin, sa dureté, son poli, sa belle couleur rouge unie, semble prédestinée pour ces divers emplois. C'est le Fou, arbre dont il existe des peuplements importants assez près de la région côtière et qui pourrait être obtenu à un prix assez bas pour être écoulé facilement en Europe. Les buis et cormiers se paient encore actuellement près de 1.000 francs le mètre

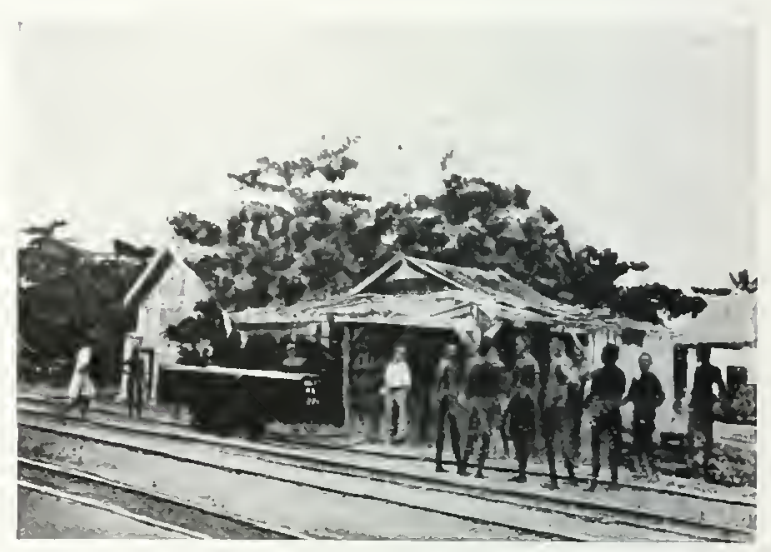

Bille pesee a la bascule de la douane. Clichè Metayer. - Grand-Bassam

cube, le gaïack, 1.600 à 1.800. Le Fou pourrait être vendu à des prix bien inférieurs (1).

Le $F_{\text {ou }}$ peut être employé également pour

nécessaire d"éliminer, en outre des bois blancs de trop faible valeur, des essences d'apparence assez belles, comme l'Ako, le Faro, l'Aninguéri, l'Anioukéli, le Sibo, qui, certainement, trouveraient leur utilisation en menuiserie ou charpente, mais qui sont, ou trop rares dans la forêt pour donner lieu à une exploitation tant soit peu importante, ou d'une conservation trop délicate. L'avenir confirmera si nous avons raison.

Et pourtant, plus on exploitera d'essences, plus on diminuera le prix de revient des bois, plus on rendra efficace, au point de vue repeuplement en essences de choix, le rôle des arbres porte-graines pouvant être réservés par l'Administration.

(1) Une maison de Montbéliard qui fait actuellement des essais de navette avec cette essence, nous a déclaré que, si lesdits essais étaient satisfaisants. elle pourrait à elle seule employer un millier de mètres cubes par an. 
des pilotis (il est imputrescible) et pour la traverse de chemin de fer.

$5^{\circ}$ Bois densité supéricure à 1.000 convenant pour la charpente lourde, le pilotis et la traverse de chemin de fer: Sougué, Tali, Adjouaba, Bodioa, Adzobé, Kroma.

Ce sont là, avec le Dabéma et le Fou, les essences classées par la mission Salesses comme bois à traverses de chemin de fer. Pour cet emploi spécial, il n y a pas d'inconvénient à ce que la liste des essences soit allongée, même si une différence de prix devait être faite entre elles. L important est que toutes soient acceptées par avance par les Compagnies. Nous aurions pu ajouter, aux six essences indiquées cidessus, le Coula, l'Adjansi et le Palétuvier. Cette dernière, notamment, donne un bois de toute première qualité pour la traverse ou le pilotis; mais guère plus que les deux autres, elle ne peut être exploitée avantageusement à la Côte d'lvoire, les peuplements étant trop peu importants, les arbres de trop faibles dimensions.

Le Sougué n'est pas seulement un bois à traverse, mais un bois de charpente ou de grosse menuiserie de premier ordre. Son importation en France, pour ces emplois, est à envisager.

Le Tali est un des plus beaux bois de la Colonie; sa dureté et sa densité limitent toutefois les emplois dont il est susceptible; nous le recommandons spécialement, en tout cas, pour la marche d'escalier et la lame de parquet. En l'assemblant avec le Fou ou l'Adzobé, qui sont aussi durs et d'aussi bonne tenue, on pourrait obtenir des parquets en mosaïques d'un très bel effet et d'une résistance à toute épreuve (1).

L'A djouaba est plutôt un bois de charpente. Nous en avons vu, cependant, d'assez jolis échantillons qui ne feraient pas mauvaise figure

(I) Le Tali est imprégné d'un alcaloïde qu est un poison violent employé par les indigènes comme poison d'épreuve. Il n'est pas à présumer cependant que ce poison puisse constituer un danger quelconque pour la confection de meubles modernes, mais c'est l'exception; la majorité des billes sont d'un gris terne. Le bois est souple et résistant; le grain est fin; il se polit facilement. Le grand défaut de l'Adjouaba réside dans le petit diamètre des arbres qui ne dépasse guère $0^{*} 50$. C'est l'essence qu'utilisent généralement les exploitants pour la confection des traineaux destinés au schlittage des bois.

Le Bodioa ne convient guère qu'à la traverse de chemin de fer. Encore est-ce un bois qui s'échauffe assez rapidement s'i] n'est débité vert et mis à sécher immédiatement.

L'Adzobé ef le Kroma sont deux bois très durs qui conviennent surtout pour la traverse et le pilotis. De même que le Fou et le Tali, ils sont inattaquables par les termites, ce qui est appréciable pour les divers emplois dont ces bois sont susceptibles à la Colonie. Ces quatre essences, considérées comme imprutrescibles, peuvent également être employées pour la traverse et le pilotis, sans être préalablement injectées de créosote ou autre solution antiseptique. Leurs facultés d'imprégnation sont du reste presque nulles : 0 kilos au mètre cube pour le Tali ef le Fou, 22 kilos pour l'Azobé, 50 pour le Kroma (1).

Les autres essences classées par la mission Salesses doivent être injectées pour être assurées d'une bonne conservation. Leurs facultés d'absorption de liquide antiseptique se rapprochent de celles du chêne (2). Dabéma, 115 kilos; au mètre cube Sougué, 70 kilos: Adjouaba, 115 kilos; Bodoia. 130 kilos (1).

pour les personnes appelées à manipuler ce bois ou à vivre dans son voisinage. (Note publiée dans la Vefice sur les bois de ta Cófe d'lioire. par le Gouvernement de la Colonie.)

(1) Expériences faites au sulfate de cuive en dissolution à 22 ". . Les bois sont plongés successivement pendant une heure dans le bain antiseptique houllant et, sans transition. pendant cing heures. dans le mème bain antiseptique froid.

(2) 80 kilos au mètre cube. Celle du hère est de 500 kilos.

(3) Voir sur les fiches d'essai de ces differents bois, leur ressistance à l'arrachement des tire-fonds. 


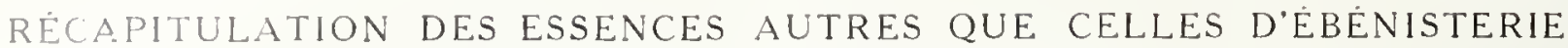

dont l'exploitation à la colonie peut être envisagée

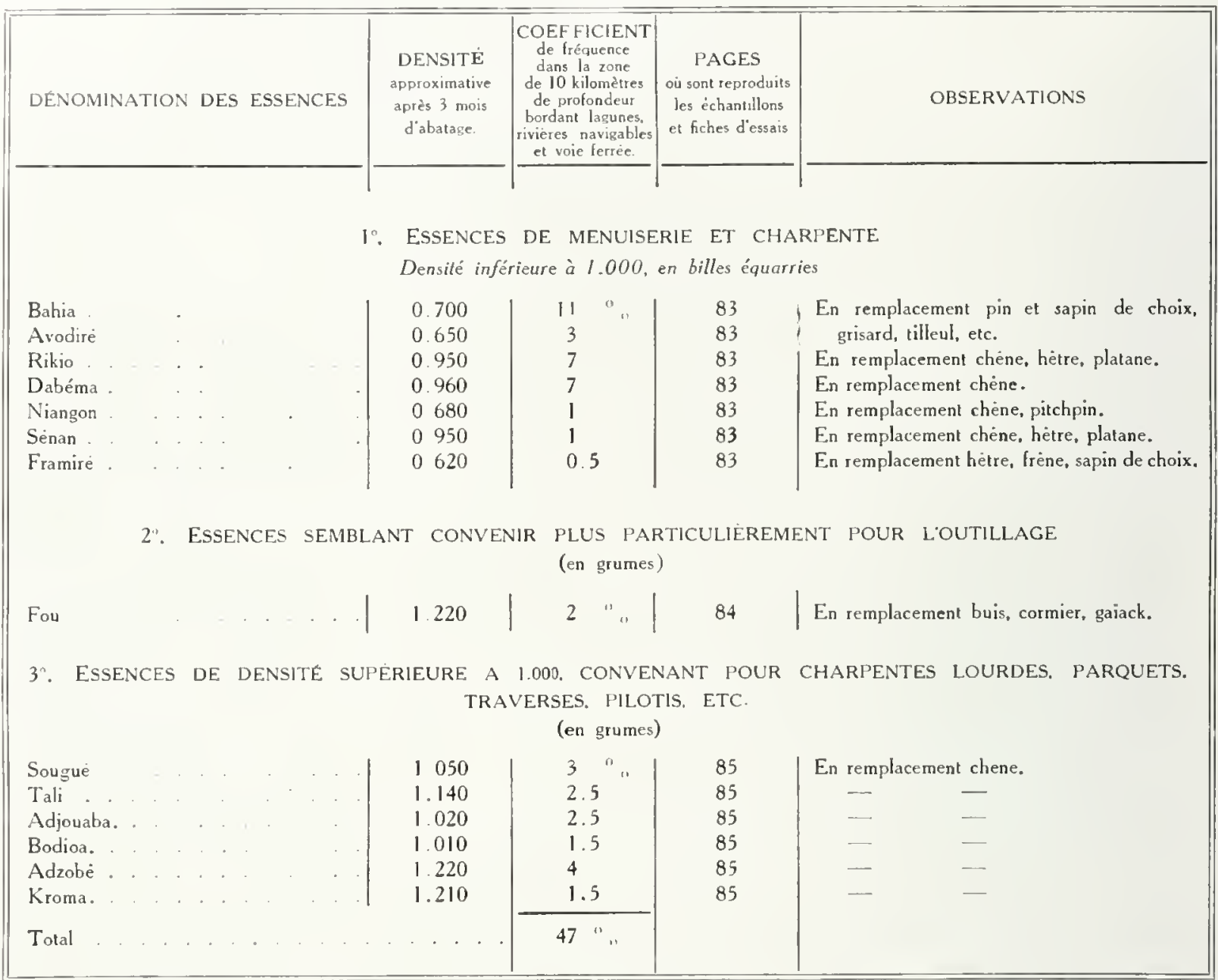

Soit 47000 du cube total de la zone de 10 kilomètres bordant les lagunes, les meilleurs cours d'eau et la voie ferrée, pourcentage auquel il y a lieu d'ajouter les bois divers d'ébénisterie que nous n'avons pas mentionnés dans ce tableau et dont l'exploitation peut n'être pas négligeable. Il y a, notamment, des peuplements d'Iroko qui sont fort intéressants. Il reste, un peu partout, malgré les coupes déjà faites, des Acajou-Tiama, Makoré, Bossé et Badi. 11 y a quelques $A$ ssas, Oboto et Olon Enfin, il y a l'A pomé que l'on rencontre fréquemment en certains endroits et dont on pourra peut-être tirer parti par la suite, ne serait-ce que pour la traverse de chemin de fer.

\section{PRÉSENTATION DES BOIS}

EXAMINÉS AU PRÉCÉDENT CHAPITRE SCIAGE ET SÉCHAGE.

\section{Présentation}

Ce n'est pas tout que de pouvoir produire des bois en plus ou moins grandes quantités. Il faut également être assuré de pouvoir les vendre et de les vendre avec bénéfice. Or, tout est à faire, tout est à organiser à ce point de vue.

Deux conditions primordiales pour que les bois de la Côte d'Ivoire, autres que les principaux bois d'ébénisterie, puissent affronter la concurrence sur les marchés de la Métropole : 


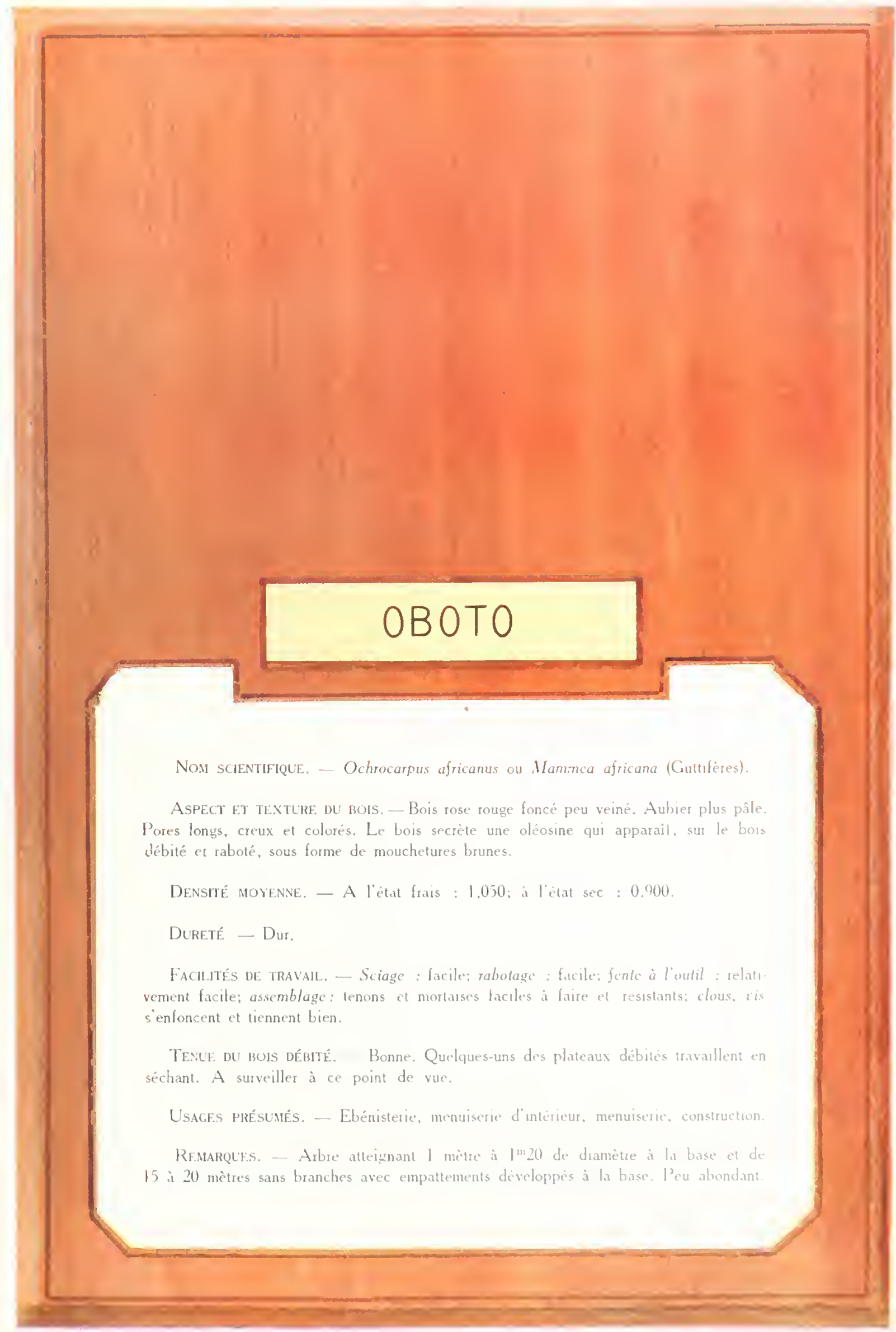



$1^{\circ}$ Etre présentés sous une forme mar. chande : plateaux, madriers, planches, bastings, chevrons, douelles, lames de parquet, ctc... débités aux dimensions couranies du commerce;

$2^{\circ}$ Avoir été séchés préalablement (1) et ne présenter aucune trace de conservation défectueuse.

Vouloir importer et écouler des bois en billes, œuvre. $\mathrm{C}^{\prime}$ est un fait reconnu pour les bois indigènes et ce n'est pas pour des bois exotiques. qui peuvent exiger, pour être débités. un outillage spécial, que les employeurs changeront de procédé. Quiconque serait tenté d'importer des bois en billes et de les vendre tels quels (1), risquerait donc, ou de ne pas les vendre ou de ne les vendre qu'à un prix très inférieur à leur

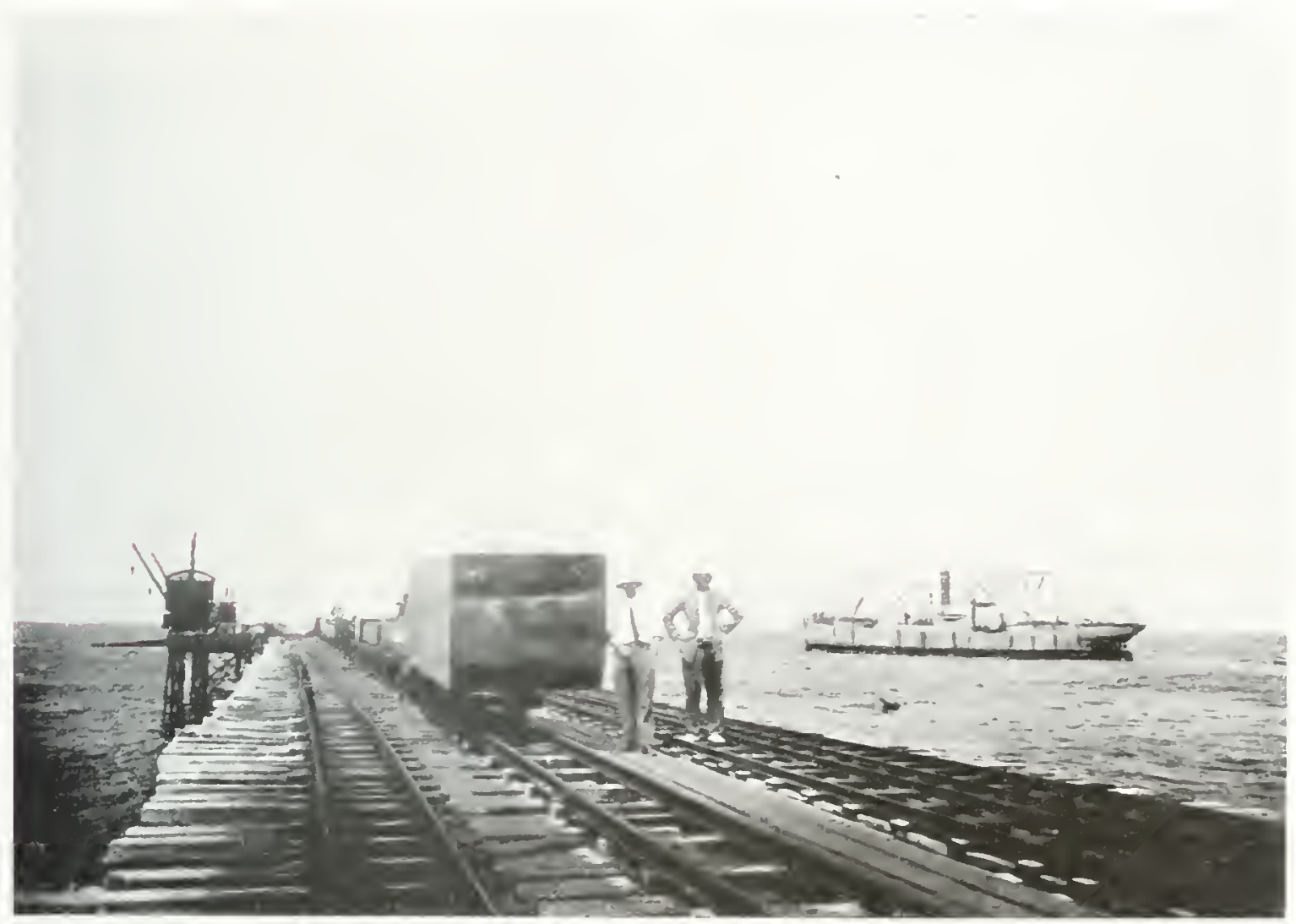

Sur le whatf. - Bille attendant son tour pour etre mise a l'eau. Clichét Mlétayer. - Grand-Barsam

équarris ou non, est aller à un échec certain. On ne les écoulerait, en effet, que très difficilement, peu d'acheteurs consentant à achetes des lots de bois qu'il leur faudrait tout d'abord faire débiter, puis conserver ensuite pendant deux ans avant de pouvoir les employer. L'employeur préfère, généralement, payer plus cher et n'acheter que des bois prêts à mettre en

(1) Sauf pour les traverses de chemin de fer qui peuvent être livrées fraîchement sciées ou deni-sèches. valeur réelle, car, seuls, des marchands de bois intermédiaires, possédant des scieries, pourraient en faire l'acquisition.

Il s’agit donc, tout d'abord. de procéder au sciage des bois. de les sécher ou desséver mécaniquement ou, encore, de les stocker dans des conditions telles qu'ils puissent sécher natu-

(1) Vous ne parlons toujours pas des beau bois de plaçage ou de contre-placagge. à trancher ou à dérouler. qu on peut très bien vendre en srunes ou equarris. 
rellement et se conserver en bon état. Il est bien certain que ce sont là deux réelles difficultés à résoudre pour les exploitants, car ces opérations nécessitent de très gros capitaü et une organisation relativement considérable.

\section{Sciage et séchage}

D'une façon générale, en France et dans les autres pays d'Europe et d'Amérique, le débitage des bois se fait à proximité du lieu d'abatage, de façon à ne pas avoir à payer le transport des déchets.

"Peut-il en être de même poúr les bois d'Afrique? " dit M. Weber (1).

Pour les bois à traverses, si celles-ci peuvent être écoulées à un prix suffisamment rémunérateur, pour tous les bois très cururs dont on aura l'écoulement en France, nous répondrons par l'affirmative. Ces bois, qui sont tous de forie densité, ne pourraient pas être mis à l'eau pour être évacués des chantiers vers les points d'embarquement, puis, de là, conduits à bord des bateaux ancrés en rade. Effectuer ces transports par chalands constituerait une opération coûteuse. Il paraît bien préférable de débiter ces bois sur place, dut-on s'en tenir, en dehors des traverses, à un premier sciage en plateaux plus ou moins épais, et de les expéalier aussitôt après, leur sciage n'étant pas indispensable pour la traverse et pouvant être effectué en France pour les autres usages. L'expédition sera plus facile et le fret moins élevé, avantages qui compensent l'inconvénient d'avoir à installer et faire fonctionner une scierie à la Colonie. Cette scierie ne devant débiter que des plateaux épais, de la traverse ou des gros bois de charpente lourde avec un nombre assez limité d'essences dures ou très dures, nécessitera un outillage spécial, il est vrai, mais néanmoins assez sommaire (2).

(1) Rapport au Conseil supérieur des Colonies, déjà cité.

(2) L'embarquement de bois sciés ne paraît guère possible qu'à Bassam. Ce n'est donc que dans la région desservie par ce port que l'installation de nouvelles scieries peut, pour l'instant, être envisagée.
Tout autrement se pose la question pour les bois de menuiscrie et de charpente légère. Nous avons vu que les scieries actuellement installées à la Colonie, si elles constituent d'assez bonnes affaires pour leurs propriétaires, ne sont telles, que parce qu'elles trouvent à écouler sur place, au prix fort et sans séchage préalable, toute leur production. Auraient-elles pu vivre si ce débouché imprévu, à peine entrevu en tout cas, au moment de leur installation, n'était entré en ligne de compte? C'est fort douteux; insuffisamment étudiées au point de vue de leur outillage, montées en pleine guerre dans des conditions très onéreuses, elles aurajent eu à affronter la lutte, sur nos marchés français, avec les provenances étrangères, dans des conditions plutôt désavantageuses pour elles.

Quoiqu'il en soit nous ne saurions conseiller, autrement que pour les bois lourds et pour les bois sujets à échauffement rapide (1), l installation à la Colonie de scieries nouvelles à très grand débit dont le rendement dépasserait de beaucoup les besoins de l'Afrique occidentale en bois débités.

Le séchage des bois, s'il est possible à la Colonie et s'il y est peut-être plus rapide qu'en Europe, exige, pour être obtenu dans de bonnes conditions, la construction de vastes hangars, absolument étanches, entièrement fermés, de façon à pouvoir atténuer, à certaines époques de l'année, l'action des vents secs qui font éclater le sciage. Et malgré ces précautions, les bois sont noircis par la grande humidité qui règne en hivernage. Ils en sont dépréciés.

Le chargement est, en outre, plus compliqué et plus coûteux pour des bois débités (qu'on doit obligatoirement conduire sur des chalands, de l'extrêmité du wharf au bord des bateaux, pour ne pas risquer de les altérer au contact de l'eau salée) que pour des billes susceptibles de flotter. L'argument invoqué contre les bois

(1) Comme le Bahia et l'Avodiré. Nous avons vu plus haut (Titre II) qu'il y aurait peut-être intérêt à scier et à desséver ces bois aussitôt après abatage. 


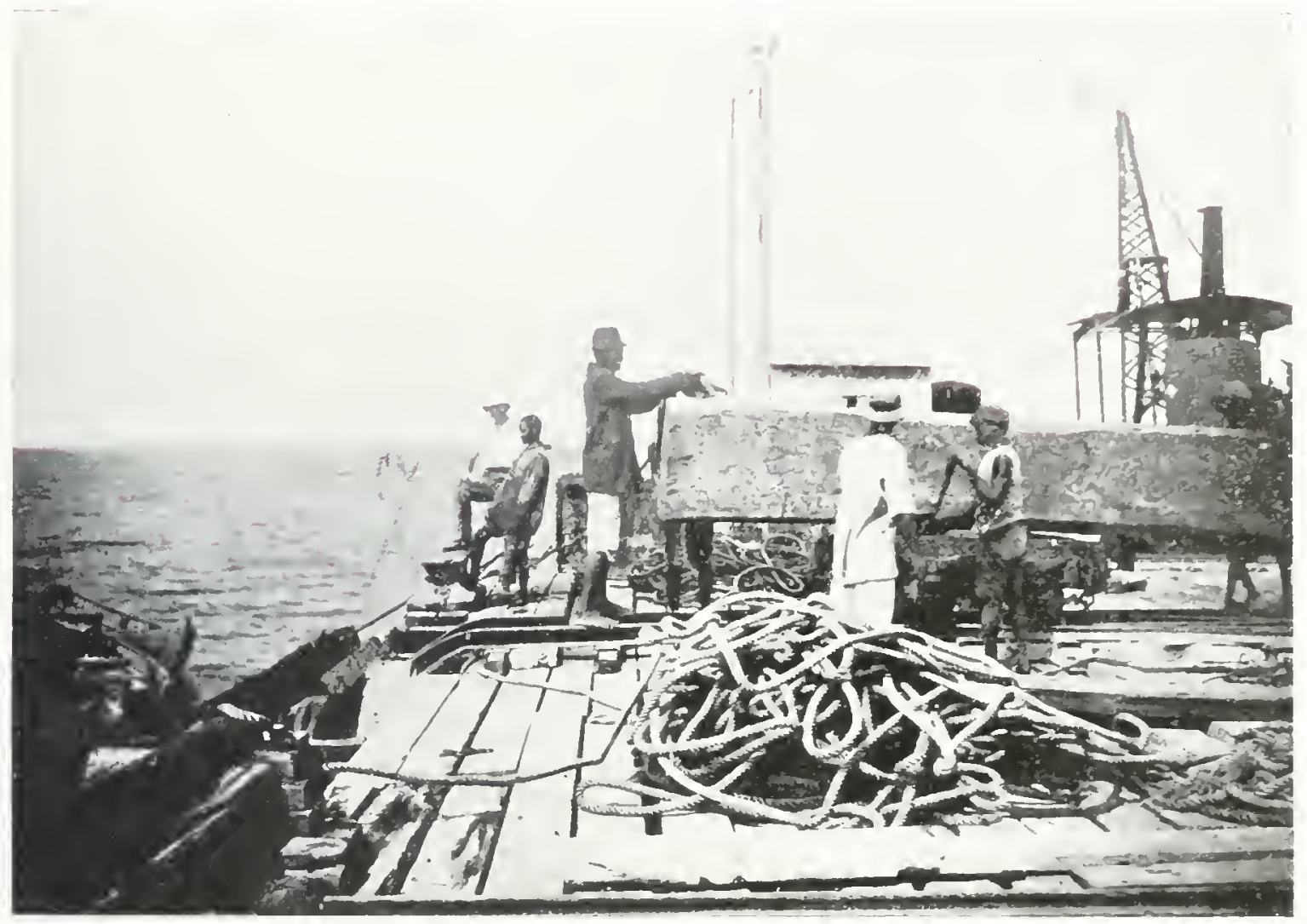

Au bout du wharf. - La bille est attachée avant d'etre jetee a l'eau.

Cliché Métayer. - Grand.Bassam

lourds se retourne donc ici en faveur des bois plus légers.

Le fret, lui-même, si l'on a pris la précaution de faire équarrir les bois, est plutôt moirs coûteux pour des billes, compie tenu du déchet à prévoir au sciage, qu il me l"est pour des bois débités (1), que les Compagnies de transport maritimes tarifient à l'encombrement, alors qu'elles acceptent généralement les billes au poids.

Enfin, il ne suffit pas de débiter et de faire sécher, il faut vendre et la seule façon d'y arriver (les acheteurs aimant à se rendre compte de la marchandise qu'on leur offre) serait de créer des entrepôts en France, d'où augmentation

(1) Nous parlons ici de planches et autres sciages commerciaux et non de plateaux ou traverses. considérable des manutentions et des frais de toutes sortes (1).

C'est donc près des centres de grosse consommation, en France (2), qu'il faut songer à installer des entreprises importantes de sciage et

(1) Inconvénient qui ne parait pas exister pour les traverses de chemin de fer, qu' on peut vendre par avance par grosses quantités el qui seront acceptées à la livraison pourvu qu'elles présentent les qualités requises (essences, dimensions, tolérance d’aubier. etc.)

(2) Il serait prématuré d'envisager de grosses installations de ce genre au Maroc. en Algerie et en Tunisie. quoique le tonnage de bois importe dans ces pars soit fort appréciable. On ne peut songer, en effet. à évincer du jour au lendemain les bois inportés d'autres pars; il faut attendre pour cela que les bois de la Côte d'lroire aient fait leurs preuves en France el soient plus connus. Des scieries de moven ou de petit débit $n$ Y réussiraient pas davantase. car elles ne justifieraient pas une exploitation speciale à la Colonie et. en achetant les bois en billes aux coupeurs. on les paierait incontestablement plus cher quils ne reviendraient à une exploitation puissamment organisée: ces scieries seraient handicapées de ce fait. 
de séchage des bois de menuiserie et charpente à importer de la Colonie; c'est là qu'il faut apporter directement les bois en billes (1), là qu'on les débitera et conservera dans les meilleures conditions possibles avec le minimum de frais. C'est également là qu'on amènera, pour les refendre aux dimensions du commerce, les bois lourds et les bois sujets à échauffement qu'on aura importés en plateaux.

La question a déjà fait l'objet de nombreuses controverses et on constatera que nous ne sommes pas d'accord avec M. Gillet, pas plus qu'avec M. Fernand Rouget (2), directeur de l'Agence économique de l'Afrique équatoriale, et l'Inspecteur Bertin (3). Nous reconnaissons, avec ces Messieurs, que la conservation des sciages aux Colonies est moins difficile que celles des grumes. Mais, nous l'avons déjà dit, nous ne conseillons nullement d'importer des grumes, mais des bois sciés en plateaux ou des bois équarris dès abatage, précaution qui assure déjà une meilleure conservation, permet d'éliminer une bonne partie des billes ayant des défauts internes que peuvent cacher écorce et aubier, et fait réaliser une èconomie sérieuse sur le fret; ensuite, il n'y a pas d'intérêt à conserver ces bois à la Colonie, mais au contraire à les expédier dès que cela est possible, au fur et à mesure qu'ils arrivent au point d'embarquement.

Installation de scierie plus facile, fonctionnement plus régulier, personnel moins coûteux

(1) Les droits de douane sont également moins élevés pour les bois en billes que pour les bois sciés. Ceux de la Côte d'Ivoire peuvent être maintenus à la classification "Bois exotiques bruts ou équarris "et être exonérés complètement.

(2) Rapport au Congrès du Génie civil, 1918. Section des Bois coloniaux.

(3) Il est bon de faire remarquer que M. Gillet, qui préconise le sciage à la Colonie, ne fait lui-même débiter les bois qu'i] exploite au Gabon qu'en plateaux d'une certaine épaisseur, opération faite à la scie de long. Le débitage est complété dans son usine de Montargis. Cette façon de procéder ne fait que confirmer notre point de vue en ce qui concerne les bois lourds; elle nenlève rien à la force de celui que nous exposons concernant le débitage des bois susceptibles de flotter. et plus facile à recruter, meilleur rendement, transport des bois pas plus sinon moins coûteux, meilleure conservation des sciages, vente possible de toute la production sans avoir à constituer d'entrepôts autres que le parc de séchage annexé à l'usine, tout milite en faveur du débiage définitif en France. La solution du problème, si on l'étudie bien sous toutes ses faces, ne peut pas être autre que celle-ci.

\section{PRIX DE REVIENT ET PRIX DE VENTE POSSIBLES DES BOIS IMPORTÉS dE la Colonie.}

La détermination de ces prix est essentielle. Les bois communs de la Colonie ne peuvent, en effet, être importés et écoulés en France que s'ils laissent un bénéfice, et un bénéfice suffisant, à l'importateur.

Nous n'envisageons pas le cas où les bois en billes devraient être achetés aux exploitants actuels de la Colonie, lesquels prendraient encore un bénéfice sur leur prix de revient déjà trop élevé.

Nos bois devront être abattus et préparés sur des chantiers organisés, d'autre part, au point de vue importation, débitage et vente.

Pour faciliter nos calculs, nous déterminerons notre prix moyen de revient des bois à la tonne et non au mètre cube, prix moyen qui pourra s'appliquer à toutes les essences. Il nous sera facile ensuite de l'établir au volume pour chacune d'elles.

$1^{\circ}$ Bois d'ébénisterie, menuiserie et charpente de densité inférieure à 1.000 au moment de leur évacuation du chantier, c'est-à-dire après un maximum de deux à trois mois d'équarrissage.

Il s'agit là des bois flottables, A cajou compris, dont nous préconisons le débitage en France, bois que nous pouvons expédier indifféremment de Bassam, d'Assinie, de Lahou, et même de Fresco, Sassandra ou San Pédro. Comme nous l'avons vu, ils seront composés 


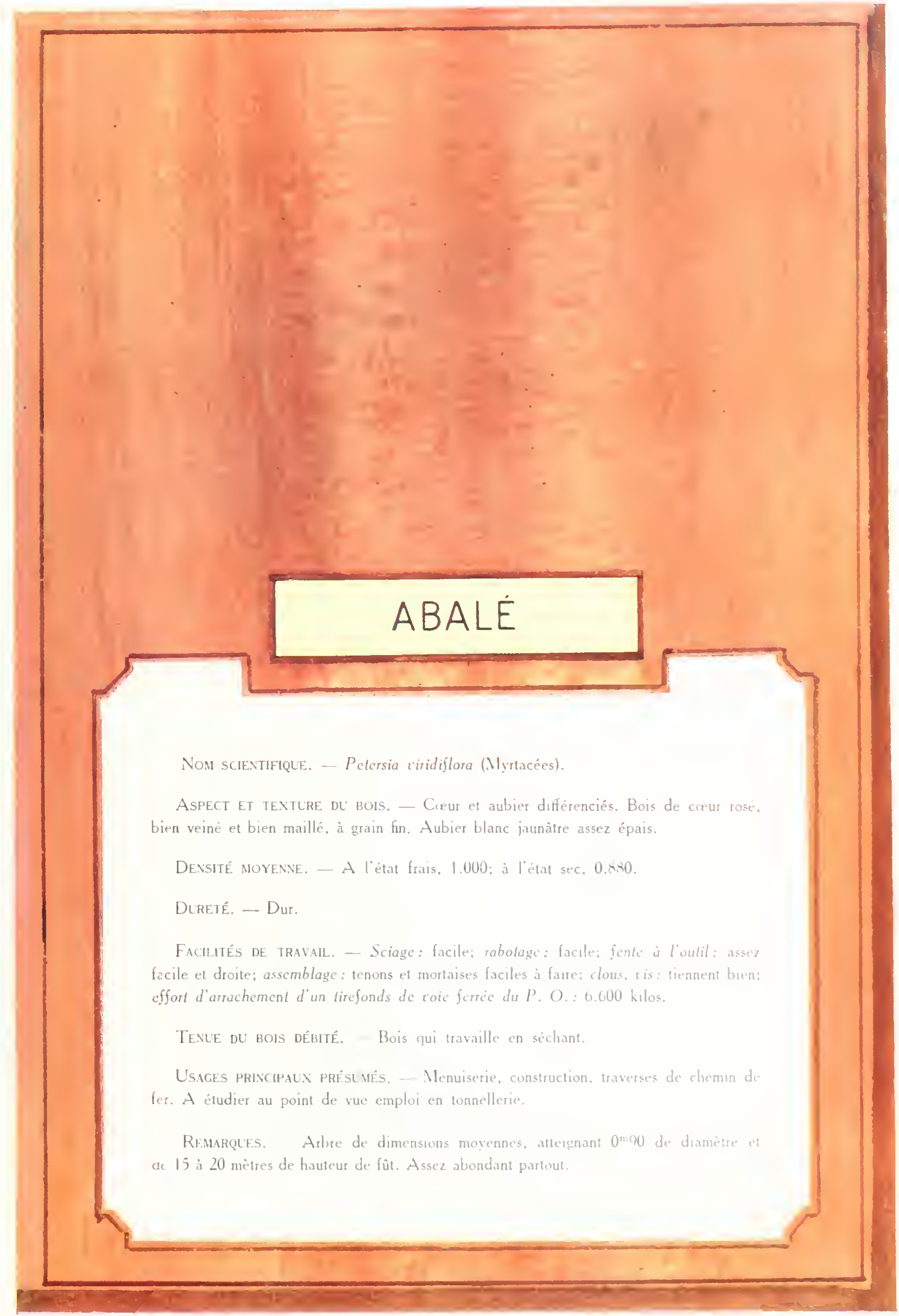


pour près des trois quarts par du Bahia, de l'Avodiré, du Rikio et du Dabéma

Personne ne contestera que le prix de revient de ces bois sera très inférieur, sur des chantiers bien placés et dont on exploitera de 40 à $600 / 0$ des peuplements, à ce qu'il peut être sur des chantiers exploités, comme aujourd'hui, plus ou moins éloignés des voies de communication, dont on sort à peine quelques centaines d'arbres qu'il faut découvrir, un par un, dans une superficie de forêt vierge de 2.500 hectares et pour chacun desquels il est nécessaire d'ouvrir des pistes de schlittage. La comparaison est même très difficile à établir. Ici, plus des trois quarts de la main-d'œuvre sont employés à la besogne d'évacuation des bois, préparation des chemins de schlittage proprement dit; quelques hommes seulement travaillent à l'abatage, au tronçonnage et à l'équarrissage. Là, au contraire, la majeure partie de cette main-d'œuvre est utilisée pour l'abatage et la préparation des billes: la grosse quantité de bois obtenue sur une surface restreinte fait qu' on n'avance que très lentement, permet l'emploi de machines (1) et d'un outillage perfectionné doublant le rendement du travail manuel; pas de chemins de schlittage à ouvrir à travers la forêt, les éclaircies, résultant des coupes, étant plus que suffisantes pour faire passer les embranchements Decauville (2) destinés à conduire les billes tronçonnées du lieu d'abatage aux voies principales; tirage à main d'homme supprimé.

Si nous nous basions, pour nos estimations,

(1) Les scies à moteur, treuils et grues à vapeur peuvent trouver leur emploi sur ces chantiers.

(2) Nous ne conseillons pas l'emploi de tanks ou de tracteurs automobiles pour ce genre d'exploitation. Quel que soit le carburant employé, essence ou pétrole qui sont très onéreux, ou même gaz paurre, qui peut revenir meilleur marché, ces machines sont de fonctionnement plus délicat que le Decauville ef ne sont pas pratiques à la Colonie où la forêt, surtout dans la zone côtière, est en beaucoup d'endroits marécageuse. Les quelques essais qui ont été tentés jusqu à présent sont peu encourageants. Encore le tracteur serait-il plus justifié pour les exploitations d'Acajor que pour celles de bois communs, où la voie Decauville peut suivre les coupeurs. sur un chantier de bois communs organisé industriellement et fonctionnant avec une régularité parfaite, nous pourrions dire qu'avec une équipe de 200 bûcherons et manœuvres, dont :

60 à l'abatage et au tronçonnage :

40 pour le chargement et l'arrimage sur wagonnets (1) ;

40 au parc de séchage, où les billes seraient équarries (2) à la scie mécanique ;

40 pour le drômage et les travaux divers :

20 pour l'entretien des voies Decauville.

Une scie à moteur pour tronçonner les arbres (ce travail peut être fait, du reste, assez économiquement au passe-partout) ;

Un treuil à vapeur monté sur wagonnet pour tirer les billeš du lieu d'abatage à l'embranchement Decauville qui arriverait à proximité (des éléments de voie volante pourraient même être posés pour conduire les wagonnets jusqu'auprès des bois abattus; dans ce cas, le treuil servirait à tirer les wagonnets chargés jusqu'à l'endroit où la locomotive pourrait les prendre) :

Une grue pour le chargement (3):

(1) Arec un ou deux crics, des leviers en bois et des chaines dacier. deux hommes chargent facilement, en France, en une heure. un arbre de deux tennes sur un chariot, généralement deux fois plus élevé que des wagonnets plate-forme Decauville. Nous tenons donc largement compte que le rendement dourriers indigènes est très inférieur à celui d'ouvriers européens.

(Par l'emploi de chèves canadiennes. on peut charger plus vite encore; une équipe de 20 à 30 hommes pourrait remplacer les animaux de trait emplovés à ce travail en Europe ou en Amérique.)

Pour le chargement sur wagonnets Decauville, des leviers à griffe doivent suffire, à condition de placer auprès des wagonnets à charger. des rondins de boi: formant tremplin. L'opération peut être faite très rapidement par ce procédé.

(2) C'est en ce point que devrait ètre installée une scierie plus complète pour le débitage des bois lourds.

(3) L'emploi de la grue à rapeur, en forèt, niest peut-itre pas très pratique. C.est une machine forcément assez lourde (elle devrait pouvoir soulever des poids de 3 tonnes) et mal équilibre sur une petite voie de 0.60 ou 0.70 d'écartement. Le ehargement sur des plateformes très basses du reste est assez vite fait par des hommes habitués à ce travail. 
Un banc de scie mécanique, scie à ruban ou scie alternative verticale ou horizontale, installé au parc provisoire de séchage (leque! parc devra être situé, soit près de la voie ferrée Abidjan-Bouaké, soit en un point des lagunes ou des rivières à partir duquel les billes pourront flotter et être conduites au point d'embarquement à n importe quel moment de l'année) ;

Une locomotive et des wagonnets en nombre suffisant,

nous pourrions arriver, facilement, à une production journalière de 50 tonnes de bois, soit 1.250 tonnes par mois, 15.000 tonnes par an.

Notre prix de revient, dans ce cas, serait excessivement faible :

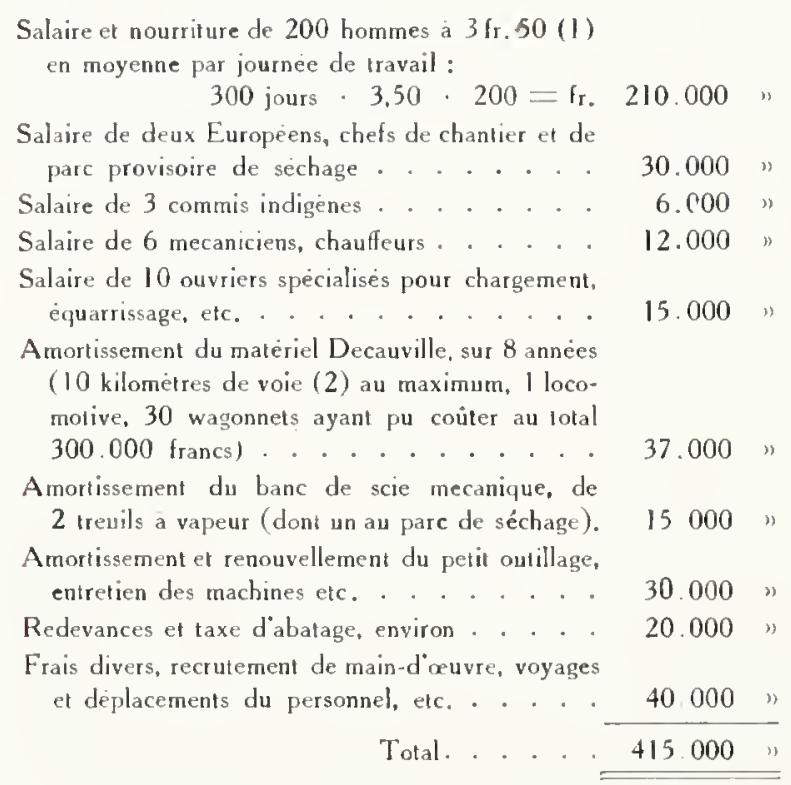

Soit 415.000 francs de dépenses pour 15.000 tonnes de bois. Prix de revient moyen de la tonne : $27 \mathrm{fr} .33$.

Nous reconnaissons volontiers que dans la pratique le rendement sera inférieur et le prix

(1) Nous aurons beaucoup de bûcherons. Mais ces 3 tr. 50 corstituent néanmoins un chiffre fort.

(2) Maximum pour une petire exploitation. Pour une production totale de 50.000 tonnes, que nous considérons nécessaire à une entreprise de ce genre, il ne faudrait certainement pas 30 à 40 kilomètres de voie, les chantiers devant être exploités à une distance moyenne de 5 kilomètres des points de concentration (pour une zone de 10 kilomètres de profondeur). de revient plus élevé, soit que le matériel employé laisse à désirer par certains côtés, soit qu'il y ait des à coups dans son fonctionnement, soit que le personnel que nous indiquons doive être renforcé.

Aussi, nous admettons que pour obtenir une production annuelle de 15.000 tonnes, les frais pourront atteindre 600.000 francs au total ou, si nos frais restent au chiffre que nous indiquons, soit 415.000 francs, que la production ne sera guère supérieure à 10.000 tonnes. Dans l’un et l'autre cas, notre prix de revient, quai Bassam, ou plage, pour les autres points d'embarquement, sera de 40 francs environ la tonne de bois équarri (1).

C'est ce dernier chiffre que nous adopterons pour nos calculs.

Comptons, d'autre part, 5 francs par tonne pour frais d'embarquement (2) et 90 francs de fret (3), prix qu'il est possible d'obtenir dès maintenant pour des quantités tant soit peu importantes, surtout en billes équarries, soit 95 francs par tonne à ajouter aux 40 francs, pour avoir le prix de revient cif port français : $40+95=135$ francs la tonne, prix approximatif.

(1) Nous parlons ici des bois susceptibles de flotter, densité inférieure à 1.000. Les bois lourds, s'il en a été coupé en même temps, seront restés à l'usine installée, comme nous l'avons dit, au point de concentration des bois, près de la ligne de chemin de fer, des lagunes ou des fleuves navigables; nous verrons plus loin le parti à tirer de ces bois.

Ce chiffre de 40 francs la tonne paraîtra, à d'aucuns, beaucoup trop faible. Pourtant on exploite actuellement au Cameroun des Palétuviers dont le prix de revient ne doit pas dépasser 25 francs le mètre cube. soit 20 francs la tonne, pour permettre de livrer des traverses de chemin de fer à 17 francs, rendues port français. Or, l'exploitation du Palétuvier n'a rien de très facile, l'enchevêtrement des racines qui émergent de 2 à 3 mètres au-dessus du niveau des eaux ou des marais où croissent ces arbres compliquant considérablement les opérations d'évacuation.

(2) Tarif du wharf de Grand Bassam, pour les bois autres que l'Acajou, le Tiama et l'Iroko.

(3) On signale même des chargements pris en novembre 1921 à 80 francs. Commencement janvier 1922 . le fret bois Bassam-Liverpool était coté en Angleterre 80-85 francs. 
Nous n'avons pas, actuellement, à payer de droits de douane pour des bois en billes provenant de nos Colonies et nous présumons qu il n'en sera pas institué.

$\mathrm{Si}$ nous voulons maintenant connaitre notre prix de revient au mètre cube, le calcul sera simple. Nous allons nous reporter aux densités indiquées à l'un des chapitres précédents pour chacune des essences recommandées (densité approximative après deux ou trois mois d'équarrissage des bois).

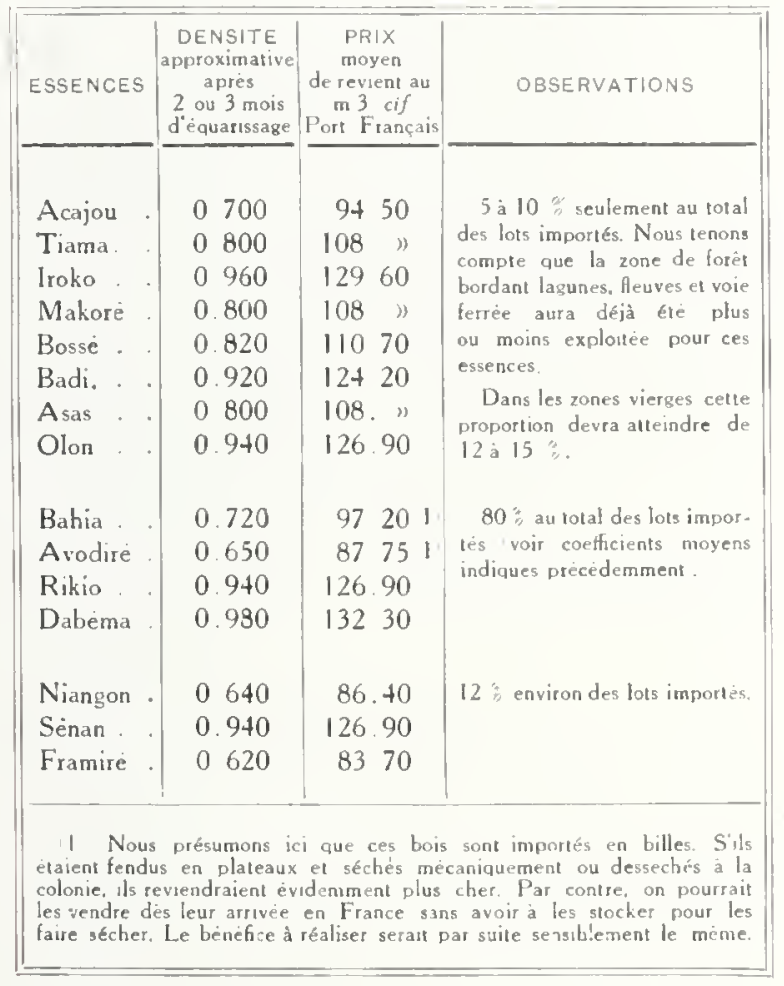

Nous obtenons ainsi un prix exceptionnellement bas pour les essences d'ébénisterie qui seront exploitées accessoirement; un prix variant de 126,90 à 132,30 le mètre cube, pour les essences dures, Rikio, Dabéma et Sénan, et de 83,70 à 97,20 le mètre cube, pour les quatre essences plus légères, Bahia, Avodiré, Niangon et Framiré.

Voyons immédiatement à quels prix pourraient être réalisés ces bois en billes, pour savoir quelle marge ils laisseraient à l'importateur pour la rémunération du capital engagé et du bénéfice de l'affaire.
Les différents bois d'ébénisterie énumérés pourraient être vendus, actuellement, en billes équarries, de 300 à 450 francs la tonne (1) cif port débarquement. Tablons sur un prix moyen de 350 francs seulement soit, pour une densité moyenne de 0.845 , au moment de leur arrivée (les bois lourds étant en majorité) 295 francs environ le mètre cube (2).

Les beaux chênes de France, grumes de diamètre supérieur à $0^{m} 50$, tronçonnés avant la première couronne, bois que le Dabéma peut facilement remplacer, valent de 260 à $300 \mathrm{fr}$. le mètre cube, un cinquième déduit, c'est-àdire, compte tenu des déchets dus à l'écorce et à l'aubier, prix s'entendant wagon départ. Il est même des chênes de Bourgogne qui se vendent jusqu'à 400 francs et plus le mètre cube.

Les chênes de qualité moindre, en belles billes, valent de 230 à 280 francs le mètre cube. Les hêtres se vendent couramment de 210 à 230 francs le mètre cube, un quart déduit; les platanes, 190 francs, le tout en billes sans nœuds, diamètre $0 " 50$ au minimum. sur wagon départ. C'est là les prix auxquels pourraient être écoulés les Rikio et Sénan, qui peuvent être employés avantageusement pour remplacer ces différents bois.

Les frênes de deuxième qualité valent, en grumes, de 240 à 270 francs le mètre cube. un quart déduit. Le Framiré trouverait probablement écoulement à 200 francs et plus; les grisards, qualité ordinaire. 150 à 160 francs. un quart déduit: le sapin du Nord ou d'Amérique. en belles billes, de 240 à 280 francs, cube réel.

Les sapins du pays ne valent que 120 francs le mètre cube (cube réel. ce qui ferait encore 150 francs au quart déduit). Nous ne voulons pas assimiler nos bois de la Colonie. en belles billes équarries, à des grumes de petit diamètre et noueuses d'un bout à l'autre. Nous ne tiendrons pas davantage compte des prix du peuplier. les moins bons des bois dont nous envisa-

(1) Début de janvier 19?1.

(2) C'est le prix des heaux chènes de menuserie. 


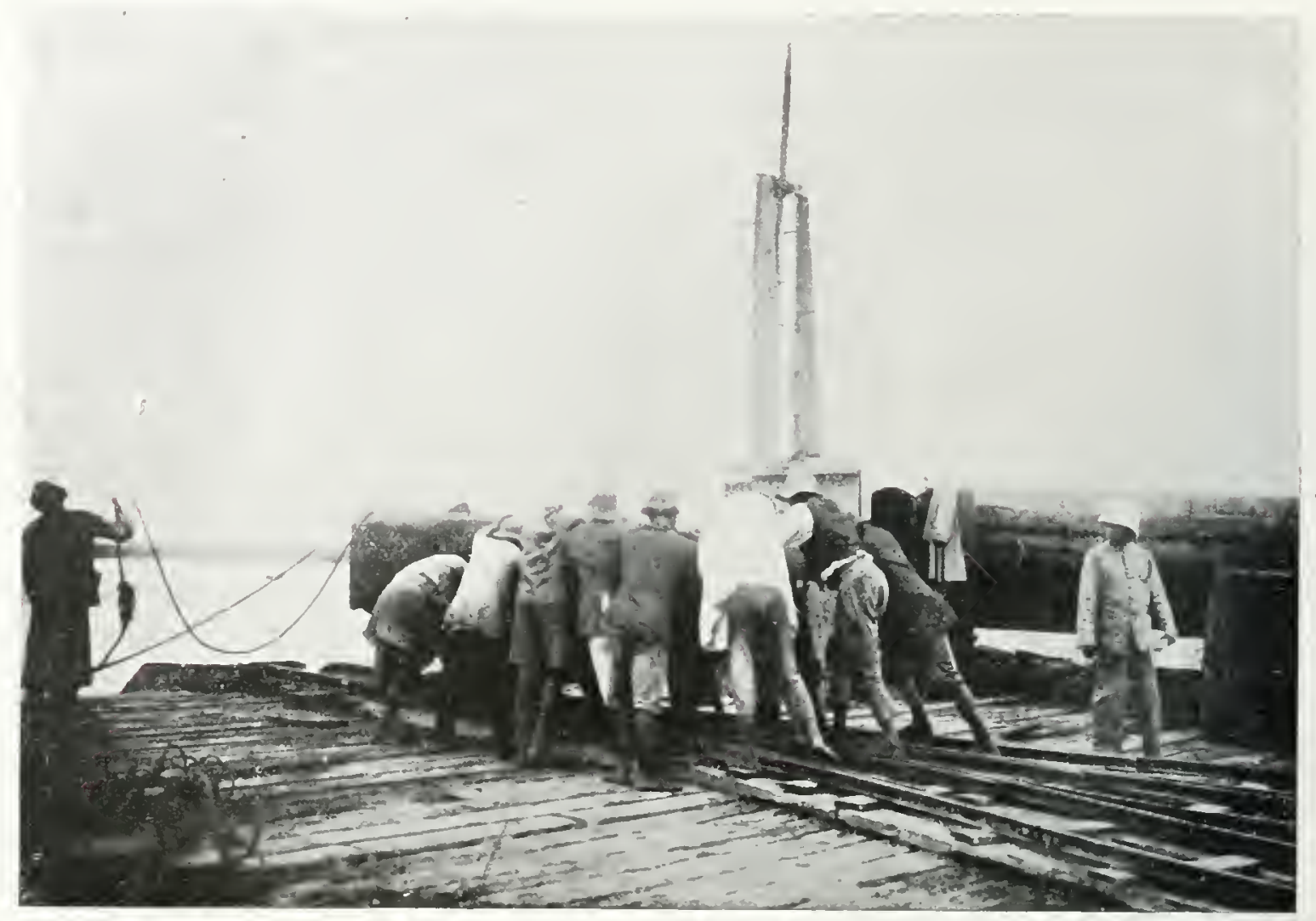

Sur le wharf. - La bille est poussee vers le tremplin.

Cliche Métayer - Crand-Bassam

geons l'importation (Bahia et Avodiré) valant les meilleurs grisards et tilleuls et les plus beaux sapins de France.
Récapitulons, pour plus de clarté, nos prix de revient et les prix de vente des bois similaires aux nôtres, comme qualité, et déduisons les

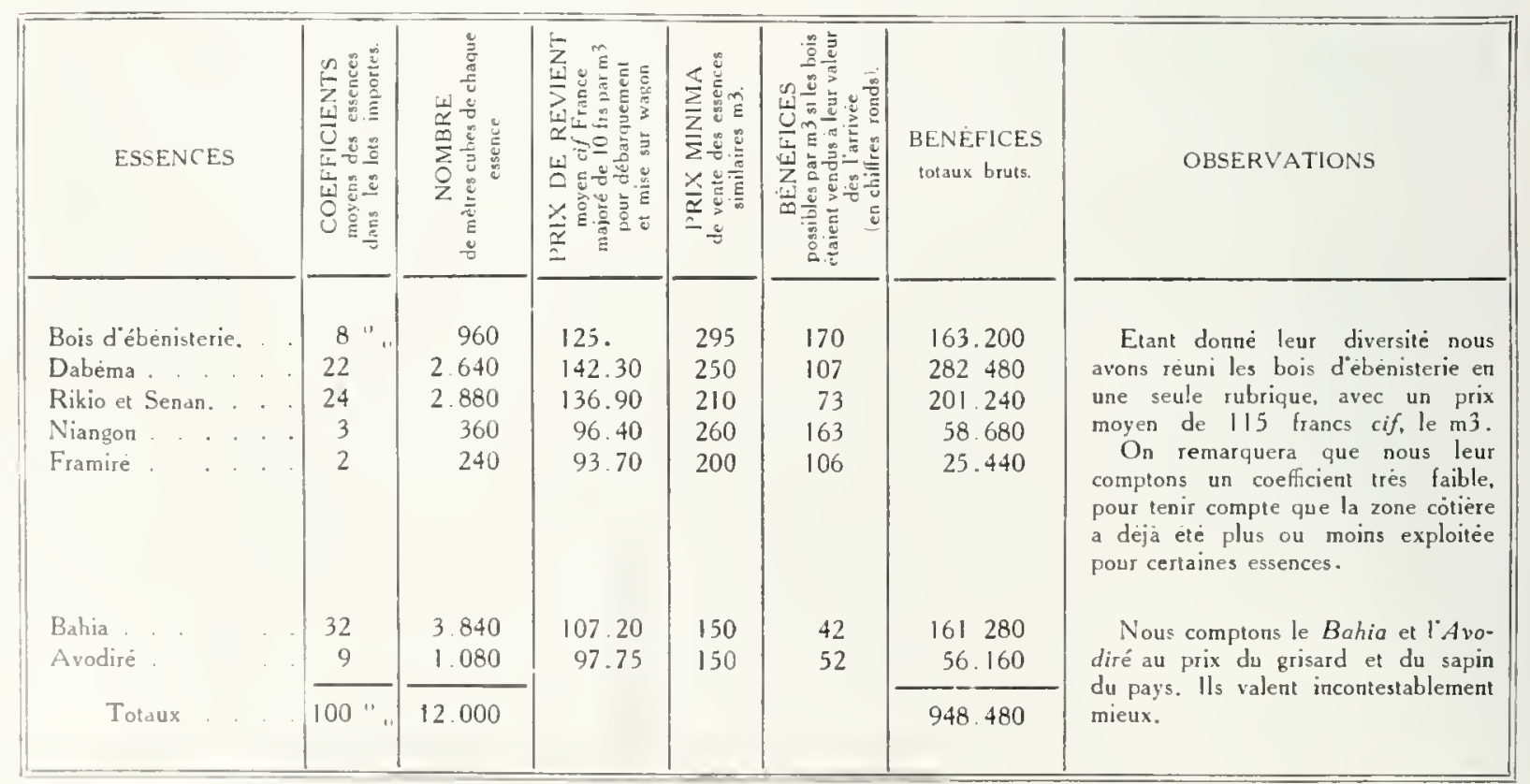




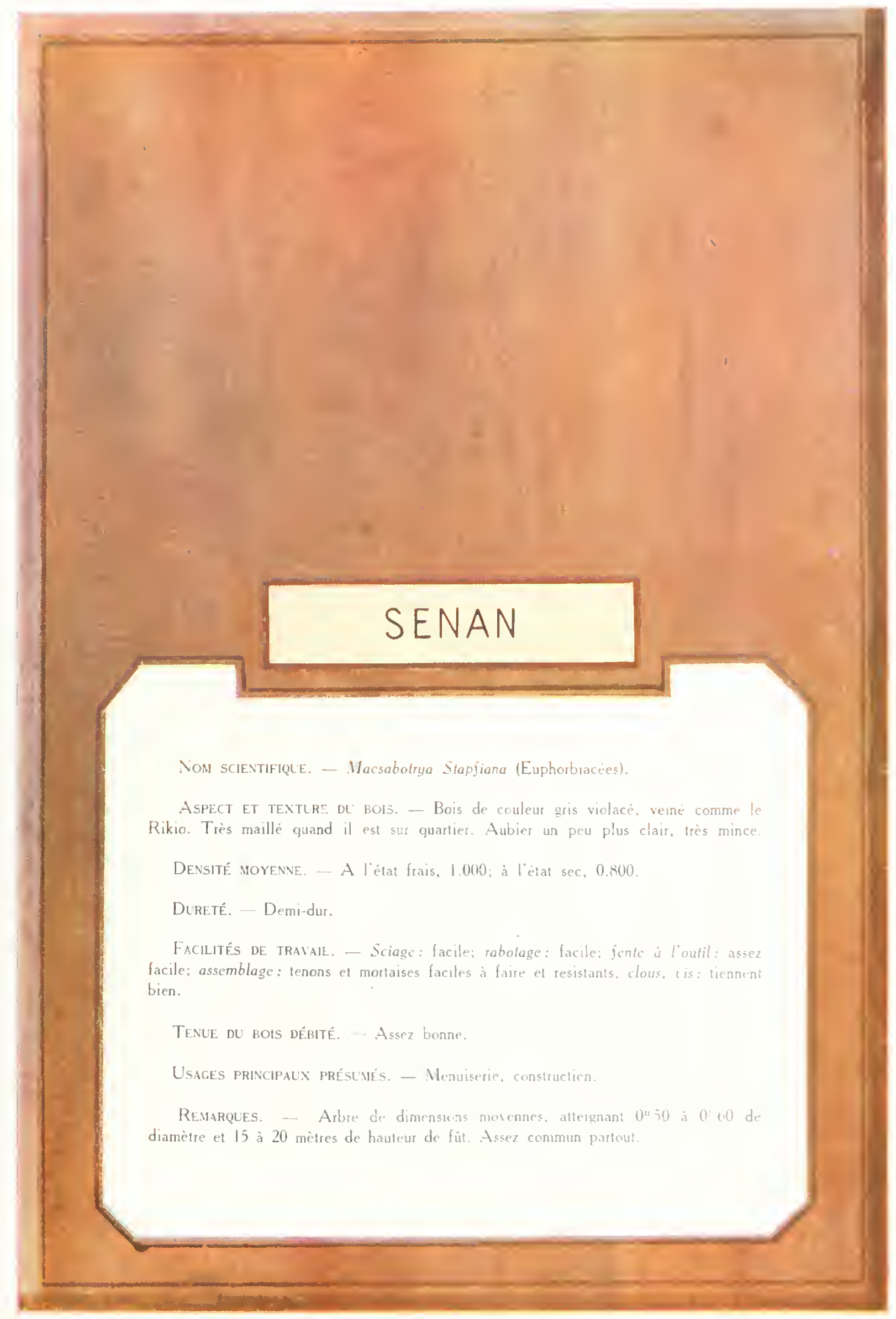



bénéfices bruts possibles qui pourraient être réalisés pour une importation de 10.000 tonnes (I) ou 12.000 mètres cubes environ.

Faisons bien remarquer qu'il ne s'agit là, aussi bien en ce qui concerne la composition des lots de bois importés, qu'en ce qui concerne l'établissement des prix de revient, partant des bénéfices possibles, que d'approximations. Les chiffres indiqués sont donc des chiffres moyens qui pourront être modifiés dans la pratique, suivant la zone de forêt exploitée, suivant les essences prédominantes de cette zone, suivant l'organisation, suivant le coût de la maind'œuvre, des frets, etc.

Le bénéfice théorique à attendre d'une exploitation annuelle de 10.000 tonnes de bois serait donc, d'après les coefficients d'essences et les prix de revient que nous indiquons, et si nos bois pouvaient être réalisés à leur valeur, dès leur arrivée en France, de près de un million de francs (2). Pour un capital engagé de 600 à 800.000 francs, au maximum, si les expéditions peuvent être faites normalement et si le fret n'est payé qu'à l'arrivée, ce serait un joli résultat.

Pratiquement, les choses n'iront pas aussi bien, en tout cas, pas aussi vite. Les bois importés ne pourront, en effet, être vendus, pour la majeure partie, qu'une fois débités et séchés, opérations qui nécessitent de gros frais, occasionnent des déchets et une immobilisation de capitaux assez considérable. Mais aussi nous vendrons nos bois prêts à mettre en ouvre à des prix beaucoup plus élevés que si nous les vèndions en billes.

$$
* \%
$$

Etablir des prix de revient pour toutes les catégories de sciage nous entraînerait trop loin.

(I) A multiplier par 5 pour une importation de 50.000 tonnes que nous considérons comme minimum nécessaire pour une entreprise de ce genre.

(2) Depuis décembre 1921. époque à laquelle cette étude a été rédigée. les cours des beaux bois d'ébénisterie et de menuiserie ont sensiblement augmenté. Les bénéfices à attendre seraient donc plus élevés. (Note de l'éditeur.)
Nous nous en tiendrons donc aux principales. Il y a du reste une relation étroite, en France, entre les prix de revient et les prix de vente des différentes catégories de bois débités, indigènes ou étrangers; il doit en être de même pour les bois de la Côte d'Ivoire et si nous pouvons prouver qu'en telles dimensions ils peuvent trouver écoulement à des prix rémunérateurs, il n'y aura aucune raison de douter qu'ils puissent soutenir la concurrence, si débités en dimensions plus grandes ou plus courtes.

Signalons, tout d'abord, qu'il est admis en France :

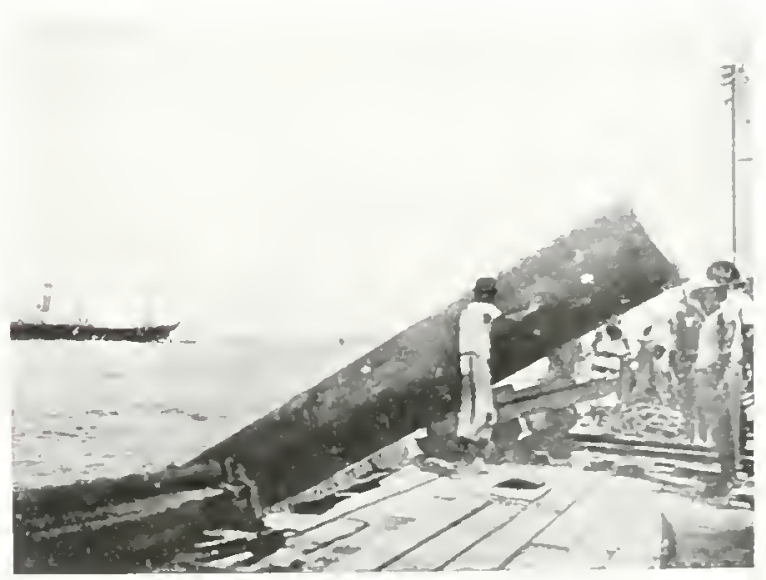

Bille glıssanı sur le ıremplın. Cliche Mléayer - Grand.Bassam

1 Que les frais de manutention des bois en billes au port de débarquement et de mise sur wagon ou péniche, peuvent s'élever au maximum à 15 francs par mètre cube:

2 Que les bois équarris à la scie (équarrissage dont nous donnons un desin-type) ne subissent qu'un déchet assez minime au débitage. soit de 8 à 200,0 (I), suivant épaisseurs, sauf pour le débit en planches très minces ou feuillets " pour lesquels le déchet peut atteindre 30 el 3500 ;

(1) $8^{\circ}$, environ pour les plateaus de 0 à $10^{\circ}$; d'épaisseur, 12 emviron pour les planches de 0.14 . 20 ". pour les planches de 0.02 et la lame de parquet. etc... 
$3^{\circ}$ Que les frais de débitage s'élèvent (tarifs industriels) de 70 à 130 francs (1) par mètre cube grume, au quart déduit, pour débits ordinaires en "plots", prix qu"il y a lieu de majorer de 20 à $250 / 0$ pour les débits en bois

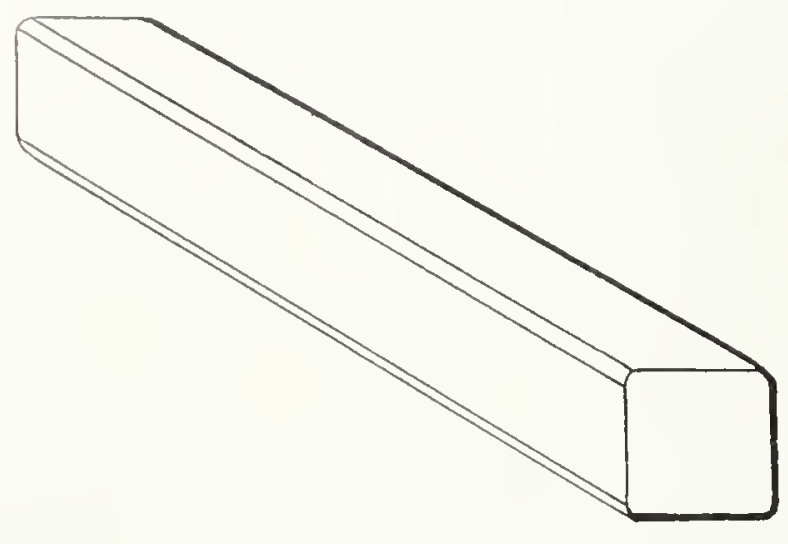

Type d’èquarrissage à prèconiser.

Billes de 4 à 6 mètres pour les bois d'ebenisterie el de menuiserie. Billes de 6 a 10 metres pour les bois de charpente.

" avivés » (2), (nos billes équarries, à l'exception des deux plateaux supérieur et inférieur qui auront un peu de fâche, seront " avivées " par avance. C'est donc les prix du débit en "plot" que nous devons leur compter. Ajoutons que les bois de la Colonie, de densité inférieure à 1.000 , se scieront aussi facilement que les bois de France);

$4^{\circ}$ Que les frais de conservation pour le séchage, en tenant compte des déchets possibles et de l'intérêt du capital immobilisé, sont calculés généralement de 12 à $150 / 0$, par année, de la valeur du bois débité et mis à sécher (3).

(1) Bois divers débités en plateaux de 0 à $120^{\mathrm{m} / \mathrm{m}}$. 70 francs; en planches de 37 à $56^{\mathrm{m} / \mathrm{m}}, 80$ francs; en planches de 27 à $36^{\mathrm{m}} / \mathrm{m}, 90$ francs; en planches de 16 à $26^{\mathrm{m}} / \mathrm{m}, 100$ francs: feuillets de 7 à $15^{\mathrm{m}} / \mathrm{m}$. 130 francs. Les grumes cubés au quart déduit donnent. en billes de plus de 0,50 de diamètre, près de $100 \%$ en moyenne de bois débité. Nos billes cubées au volume réel ne donneront que 80 à $92 \%$. On devrait donc pouvoir les débiter à meilleur compte.

(2) C'est-à-dire sans Alâche. Suivant les catégories de bois, l'aubier est enlevé ou non.

(3) Nous avons obtenu tous ces renseignements aulprès de sources sûres. Nous les avons recoupés plusieurs fois. De même pour ce qui concerne les prix actuels de vente des sciages que nous indiquons plus loin.
Majorons en conséquence de 20 francs par mètre cube (1), dont 15 francs pour frais de débarquement et manutentions diverses et $5 \mathrm{fr}$. pour rémunération normale du capital engagé jusqu'au moment où les bois peuvent arriver en France (12.000 mètres cubes, soit 60.000 francs pour 800.000 francs de capital engagé au maximum) et établissons, d’après les

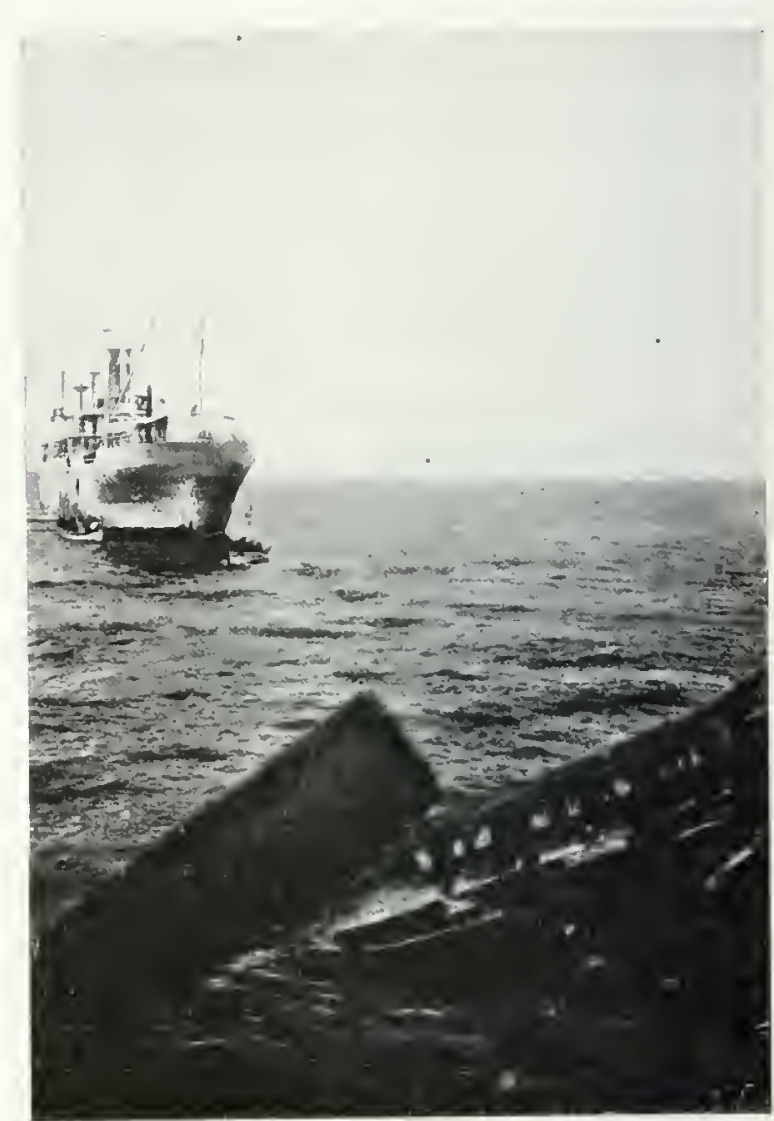

Bille quittant le tremplin. Clıchè Métayer. - Grand-Bassam

données ci-dessus, le prix de revient moyen de nos bois, débités et séchés, prêts à être employés.

Or, que valent actuellement les sciages secs sur nos marchés, sciages de bonne qualité, sans aubier ou presque, et sans nœuds et provenant de troncs assez volumineux (diamètre supérieur à 0.50 ).

(1) Réguljèrement nous aurions dû compter davan. tage pour les bois les plus lourds, et réduire pour les essences légères. 


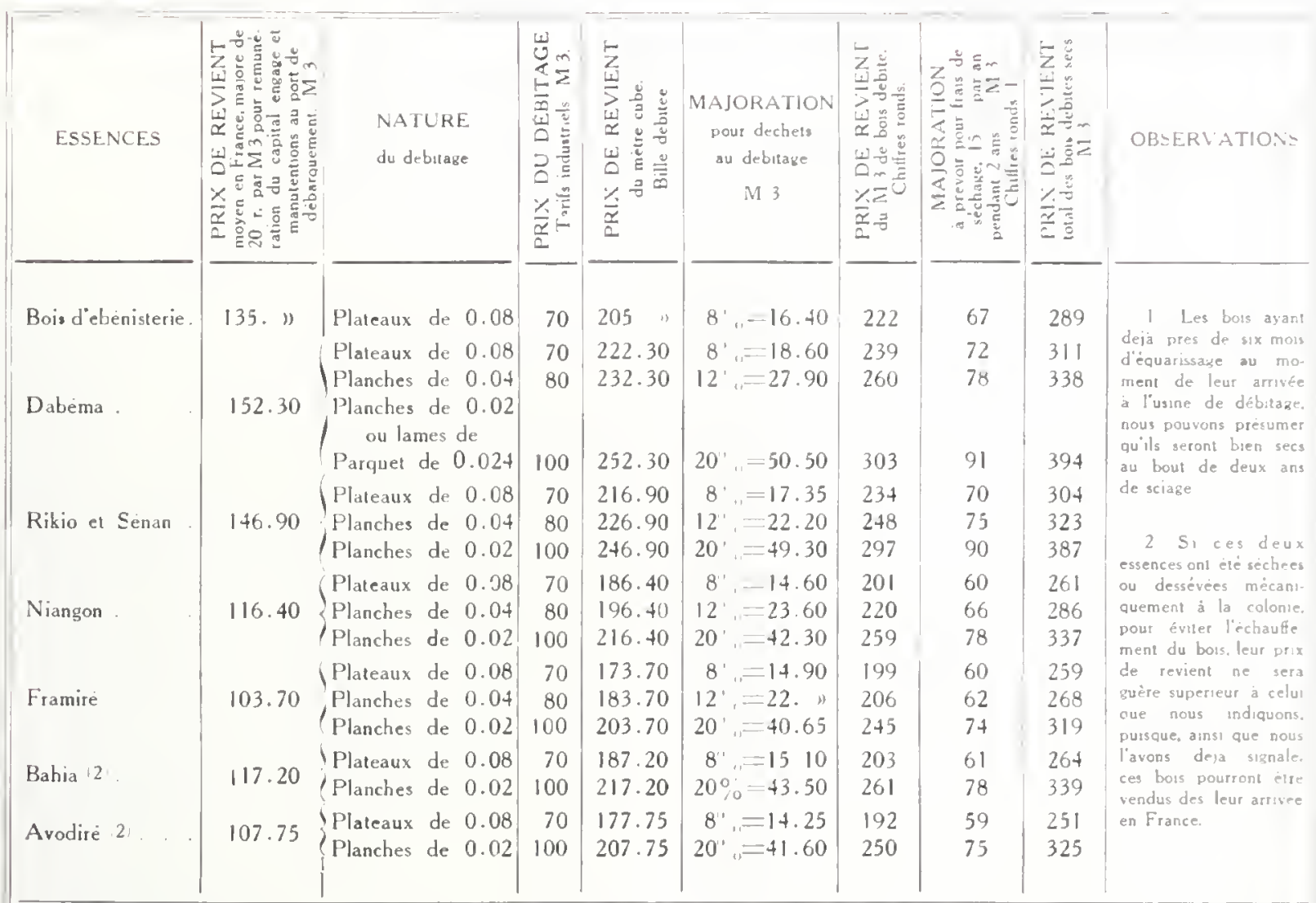

NOTA - Une partie de ces bois pourra ètre vendue aussitot après sciage aux industriels lenani à faire sécher eux-mêmes leurs bois ou dısposanı des installations nécessaires pour l'étuvage ou le séchase mécanique. Les essences de la Côte d’lvorre se comportent aussi bien. soumıses à ces opératıons. que lés bois indigenes.

Le séchage artificiel n'est toutelois pas ä préconiser pour la plupart des bois d'ébenistefie: leur couleur peut, en effet, en ètre altéree

Nous avons obtenu, de différents côtés, des renseignements assez précis à ce sujet :

Sans parler des bois d'ébénisterie de grand choix, qui se vendent couramment de 1.000 à 1.200 francs le mètre cube, et des chênes du Japon, de Hongrie, de Pologne, de Yougo-Slavie ou des Etats-Unis, qui sont écoulés à des prix variant de 650 à 900 francs le mètre cube, nous avons, pour les bois plus communément employés en France, les chiffres suivants, qui s’appliquent à des bois de qualités diverses.

\section{Bois d'ébémisterie (1)}

Acajou ordinaire Bassam, sec. en platerux de $0^{\mathrm{m}} 08$, bois avive : le metre cube . Noyer ordinaire, plateaux de $0^{m} 08$, bois avive:

le metre cube. . . . . . . . avive : le metre cube. . . . . 550 a 650 "

(1) Prix moyens, début janvier 1921.

\section{Chène, qualité menuiserie}

a) En "plots" (Alache compris) (1):

$$
\begin{aligned}
& \text { Plateaux de } 60 \text { a } 120 \text { ". le metre cube } \$ 25 \text { a } 550 \mathrm{fr} \\
& \text { Planch s de } 27 \text { a } 54 \% \text { - } \\
& \text { - de } 10 \text { a } 22 \% \text { - } 150 \text { a } 575
\end{aligned}
$$

b) En debits, largeur variable bois avise:

Plateaux de 60 a $120 \%$. le metre cube 475 a 000 frs

Planches de 27 a $54, \quad-\quad 500$ a 700

Feuillets de 7 a 22 , $\quad$ - 850 a 1.250

Lames de parquel de $2 t^{\circ}$ " _ $\quad$ - 600 a 700

3 Chene, qualite charpente (bos anve)

En plateaux de $0 \mathrm{~m}_{08}$, le metre cube. . 350 a 450 fr En planches de $0{ }^{m} 02$. - . . 400 a $\$ 50$

(1) On appelle Aache n dans l"industrie du bois. la partie d'un plateau ou d'une planche joignant l'écorce et qu'il est nécessaire d'enlever à la scie pour obtenir une pièce " avivée ". c'est-à-dire à angles droits, vifs. Pour les grumes qui ne sont pas absolument droites, l'enlèvement du flàche peut laire subir un assez gros déchet.

Le débit en "plots ". consisie à fendre les grumes en plateaux ou planches de dilférentes epaisseurs dans toute la largeur du bois: l'écorce et l'aubier ne sont pas enlevés des pièces sciées. 
4. Hêtre, qualité carrosserie

En "plots" (Aâche compris):

Pla'eaux de 60 a 120 "m. le metre cube 300 a $350 \mathrm{frs}$

Planches de 27 à $54 \mathrm{~m}$, — 325 a 375 "

Feuillets 15, 18 et et $22 \mathrm{nin}$.

5. Hêtre, qualité menuiserie

En largeurs variables (bois avive):

Plateaux de 60 a 120 m. le metre cube 350 a 475 frs

Planches de 27 à 54 "in, - $\quad 375$ a 525

- de 15,18 et $222^{\mathrm{m}}$. - 475 à 750 ,

6" Frène de deuxième qualité

a) En "plots" (Hache compris):

Plateaux de 60 a $120 \mathrm{~m}$. le metre cube 350 à 400 frs

Planches de 27 a 54 "

b) En bcis avives :

Plateaux de 60 a 120 "'n. le métre cube 400 a 500 "

\section{7" Platane}

En bois avive :

Plateaux de 60 a $120 \mathrm{~m}$, le metre cube 270 a 300 frs

Planches de $20^{\mathrm{m}} \mathrm{m}$. . . - $\quad 290$ a 350 ,

$8^{\circ}$ Grisard de bonne qualité moyenne (en demi-sec)

En largeurs variables (bois avives):

Planches de 10 a 25 "'... le metre cube 220 à 280 frs

9" Tilleul ef charme (en demi-sec)

En "plots":

$$
\text { Plateaux de } 60 \text { a } 115^{\mathrm{in}} \text {. le metre cube } 225 \text { frs }
$$

En bois avives:

Plateaux de 60 a $115^{\mathrm{m}}$, le metre cube 240 a 300 "

\section{0" Pitchpin}

Madriers pleins de 23:8.. le métre cube 250 a $350 \mathrm{frs}$ - 2 traits.... - - 320 a 400 " Planches de 30 a $50 \therefore 10{ }^{m} \quad-\quad 350$ a 450 "

$11^{\circ}$ Sapin du Nord ou d'Amérique, qualité menuiserie

Madriers........

Bastings . . . . . le mètre cube 450 à 500 frs

Planches

12. Sapin Snède, qualité charpente

Madriers

Bastings . . ( le mètre cube 300 à 400 frs

Voliges

1.3. Sapin de pays, tout renant

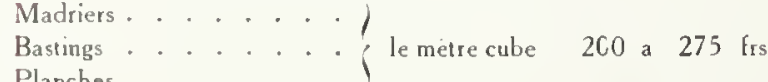

Planches

Nous voyons que, sauf en ce qui concerne les sapins du pays, provenant généralement de troncs noueux d'assez petits diamètres, nos bois de la Côte d'lvoire peuvent concurrencer avan- tageusement toutes les autres essences et laisser à l'importateur une assez belle marge de bénéfices.

Les bois d'ébénisterie revenant à 290 francs en plateaux, pourront être écoulés à des prix allant de 500 à 800 francs le mètre cube.

Le Dabéma, qui nous reviendra de 300 à 400 francs le mètre cube suivant dimensions, doit pouvoir trouver acquéreurs aux prix des chênes de menuiserie, soit (bois avivés) de 475 à 700 francs le mètre cube.

Les Rikio et Sénan, pouvant remplacer les chênez de charpente et les beaux hêtres (bois avivés) nous reviendront de 300 à 390 francs suivant épaisseurs, et pourront certainement être vendus de 350 à 500 francs le mètre cube.

Le Niangon et le Framiré, dont les coefficients ne seront malheureusement pas très élevés, pourront trouver écoulement à des prix très avantageux, supérieurs certainement à 400 francs le mètre cube.

Le Bahia est, probablement, celui de nos bois qui donnera les moins bons résultats. Il sera nécessaire d'en faire des billes assez longues pour que les sciages puissent, le cas échéant, être employés pour remplacer les bois de charpente du Nord ou d'Amérique et trouver écoulement, pour la majeure parie, à des prix allant de 300 à 400 francs, suivant débit; le moins qu'on puisse obtenir pour les sciages plus courts, ce sera les prix du grisard et du tilleul, soit de 220 à 280 francs pour des bois demi-secs, ce qui couvrira encore largement le prix de revient des autres bois. Dut-on ne rien gagner sur cette essence, qu'il serait néanmoins nécessaire de l'exploiter pour réduire le prix de revient des autres bois dont nous conseillons l'importation.

L'Avodiré est de meilleure qualité, à notre avis, et doit pouvoir être vendu à des prix supérieurs à ceux du Bahia. L'une et l'autre de ces essences peuvent du reste être obtenues en billes assez volumineuses pour permettre le débit de panneaux larges et droits pouvant être recherchés pour la menuiserie. Ces deux bois se travaillent également assez bien et peuvent trou- 


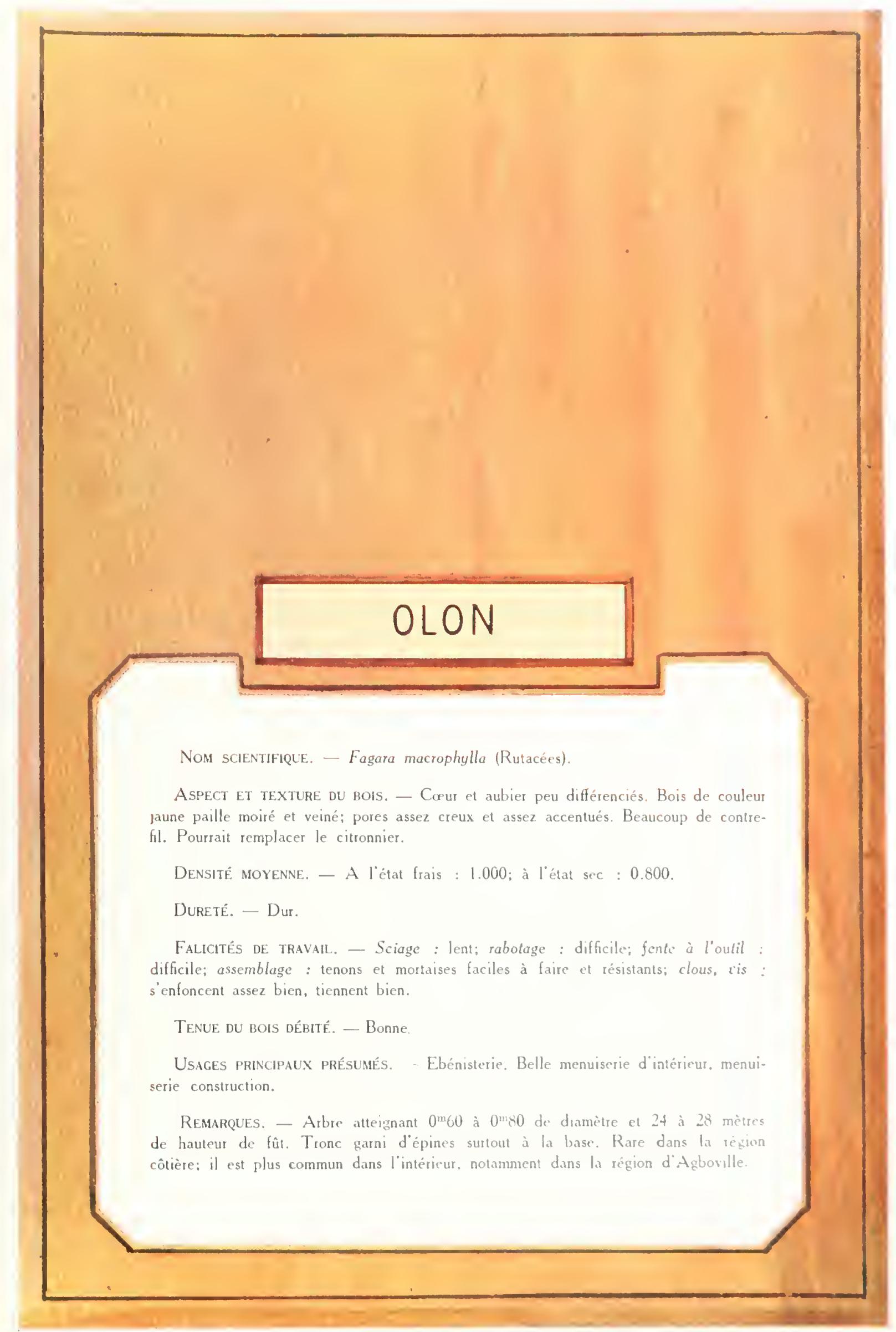



ver, pour la moulure, des débouchés intéressants.

$2^{\circ}$ Bois de densité supéricure à 1.000 au moment de leur évacuation des chantiers, bois utilisables pour l'ébénisterie, le parquet, l'outillage, la grosse charpente, la traverse de chemin de fer, le pilotis, etc.

Les différents bois lourds que nous avons passés en revue à l'un des chapitres précédents reviendront, šils sont exploités en même temps que les bois plus légers dont nous venons d'étudier les possibilités d'écoulement, sensiblement

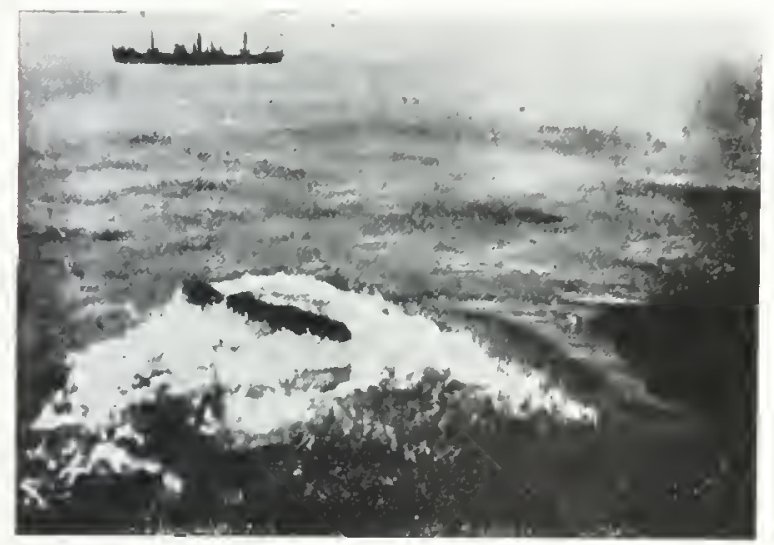

La bille revient a la surface. Cliché Métayer. - Grand.Bassam

moins cher à l'usine de débitage installée à la Colonie que ces derniers bois ne peuvent revenir au point d'embarquement, soit 40 francs environ la tonne d'après le décompte que nous avons fait plus haut et qui prévoit largement tous les frais d'exploitation. L'équarrissage sera inutile. Le point de concenfration sera pour eux le point d'aboutissement. La scierie sera placée. en effet, comme nous l'avons exposé, au débouché des chantiers, soit le long de la voie ferrée Abidjan-Bouaké, soit, de préférence, auprès d'une des lagunes ou des rivières navigables, de façon à être reliée directement à eux par le chemin de fer Decauville qui aura été placé pour l'évacuation des différents bois abattus.

De cette façon, nos bois lourds n'auront à voyager sur l'eau qu'une fois débités, placés dans les chalands qui les conduiront à Bassam pour l'embarquement.

Ces bois ne nous coûteront donc guère que $32-35$ francs la tonne, prix moyen. Toutefois, comme leur densité varie à l'état vert, de 1.000 à 1.250 , comptons le prix de 40 francs sus-indiqué comme prix moyen de revient du mèlre cube à l'usine de débitage.

Examinons d'abord la question traverse de chemin de fer :

Le déchet des grumes, au débitage, n’est pas considérable pour les traverses, 10 à 12 tout au plus, si les billes ont été tronçonnées à la longueur voulue (1). Un mètre cube de bois doit nous donner facilement onze traverses du type adopté par nos Compagnies de chemin de fer, soit $2^{\prime \prime} 60 \times 0^{\prime \prime} 26 \times 0^{\prime \prime} 13$, avec tolérance d'aubier et angles face supérieure chanfreinés ou arrondis (2).

Le débitage en traverses est beaucoup plus rapide que celui en planches de différentes épaisseurs. Estimons toutefois, pour tenir compte qu'il sera effectué à la Colonie et qu'il s'agira de bois durs ou très durs à scier, un chiffre de frais s'élevant au total (amortissement usine compris) à 70 francs, en moyenne, par mètre cube. C'est même un maximum, d'après les renseignements qui nous ont été fournis. Nos onze traverses nous reviendront donc, à la sortie de l'usine, à 40 plus $70=110$ francs. Ajoutons à ce chiffre les frais d'embarquement 10 francs, le fret 90 francs (3) et nous aurons un prix de revient cif, port français, qui ne sera pas infërieur à 200 francs. L'importateur, pour les vendre avec bénéfice, ne pourrait guère les céder à moins de 250 francs, soit près de 22 francs la traverse (4). Trouvera-t-il des acheteurs à ce prix?

(I) $2^{\text {n }} .60$ ou multiples de $2^{\prime \prime .60}$ pour la France; $1^{\text {"'. }} .80$ ou 2 mètres pour les chemins de fer coloniaux.

(2) Voir ourrage C BERTIX, déjà cité. pages os? et suivantes.

(3) Prix quion pourrait sans doute ohtenir dès maintenant pour des quantités inportantes.

(4) 24 francs même, si l"on tient compte des droits de douane applicables aus bois débites senant de la Colonie. (l fr. par l 100 kilos.) 
Nous sommes bien obligés de répondre par la négative. Les grandes Compagnies paient, en ce moment, la traverse de hêtre $12 \mathrm{fr} .50$ et la traverse de chêne 15 à $15 \mathrm{fr} .50$; l'une et l'autre reviennent, une fois injectées de créosote (la première en absorbant beaucoup plus que la seconde), à un prix "voisin de 17 francs. 17 fr. 50. Or, les Compagnies ne consentent pas à payer plus que ce dernier prix les traverses coloniales, même celles de bois considérés comme imputrescibles et gui peuvent se passer d'injection antiseptique (1).

De 17 à 24 francs la différence est trop grande pour qu'on puisse envisager l'importation, même si une réduction très sensible des tarifs de fret devait se produire par la suite. Faut-il en déduire que la question traverses ne peut présenter aucun intérêt pour les exploitations forestières de la Côte d'lvoire? Pas si vite, car si nos traverses ne peuvent être écoulées en France, elles peuvent trouver des débouchés ailleurs.

Prenons d'abord l'Afrique occidentale française. On a construit jusqu'à présent les chemins de fer existant dans cette Colonie (exception faite pour le Dakar-Saint-Louis) avec des traverses métalliques qui, actuellement, reviennent extrêmement cher. 1 l n'est pas question de remplacer celles qui sont là par des traverses en bois. Mais il reste beaucoup de chemins de fer à construire en Afrique occidentale française. Le programme Sarraut en prévoit plus de 800 kilomètres pour lesquels il faudra 1.200.000 traverses. Cela nous représente déjà, à 20 traverses environ au mètre cube (2) 60.000 mètres cubes de bois à débiter en huit ou dix ans. Le Maroc a importé, en 1920, 8.800 tonnes de

(1) Un marché assez important a été passé récemment avec une maison installée au Cameroun pour la fourniture de traverses en palétuvier. Le prix fixé fob Duala a été calculé de façon qu'avec le fret, les droits de douane ( $1 \mathrm{fr}$. par traverse) et les frais divers, ces traverses reviendront à 17 francs environ port débarquement. Une opération semblable ne paraît pas possible actuellement avec les bois de la Côte d'lvoire.

(2) Pour voies de I mètre. traverses, l'Algérie 15.000 tonnes, la Tunisie 3.600 tonnes, importations qui iront augmentant, car il faut non seulement construire, mais remplacer peu à peu les traverses posées. Or, dans ces différents pays, le prix de vente possible peut être calculé sur le prix de France (compte tenu de la différence de volume des traverses), majoré des frais d'embarquement et de fret jusqu'au port de débarquement. Nous ne serons donc pas embarrassés pour vendre 250 francs le mètre cube à Dakar, Konakry ou autres points de la Côte, ce que nous pourrions vendre, plus difficilement, 190 francs en France; nos frais de transport seront en outre moins élevés. Pour le Maroc, l'Algérie et la Tunisie, la question est à étudier de très près, mais il ne serait pas impossible que nos traverses puissent s'y vendre également avec profit pour l'importateur.

Mais nos bois lourds de la Côte d'Ivoire peuvent trouver, en Afrique occidentale française, des débouchés autres que la traverse de chemin de fer, notamment pour le pilotis, les travaux de charpente en plein air, la construction, etc.

Enfin, toute la meilleure partie des belles billes d'Oboto (bois d'ébénisterie), de Fou (bois pour outillage), de Tali (bois pour parquets, escaliers, etc.) d'Adjouaba et autres pour lesquels on aura trouvé une utilisation dans la Métropole, sera débitée en plateaux d'épaisseur variable, plus maniables que des billes entières et dirigées vers l'usine montée en France pour y être mises à sécher et, le cas échéant. débitée à nouveau.

Ces plateaux de bois de choix reviendront un peu plus cher que les traverses de chemin de fer car nous aurons enlevé tout ce qui est aubier au débitage et les déchets seront plus considérables. Comptons environ 230 francs le mètre cube, cif port débarquement et, avec les frais de débarquement, de douanes, de manutentions diverses, un prix de revient maximum à l'usine ou au parc où ils seront mis à sécher, de 260 francs le mètre cube.

L'importateur ne sera pas embarrassé pour les 
vendre à un prix double et peut-être triple. Nous avons vu, en effet, que les beaux chênes débités en plateaux se vendent couramment de 475 à 600 francs le mètre cube sur wagon départ. Et des bois comme le Tali ou l'Oboto valent mieux que nos plus beaux chênes, pour certains usages.

Les chênes pour parquet, qualités très moyennes de bois, se vendent de 15 à 18 francs le mètre carré, en 24 millimètres d'épaisseur, soit de 590 à 740 francs le mètre cube. Nous voulons croire que du parquet de Tali ou d'Adzobé se vendrait au moins au même prix. Or, pour

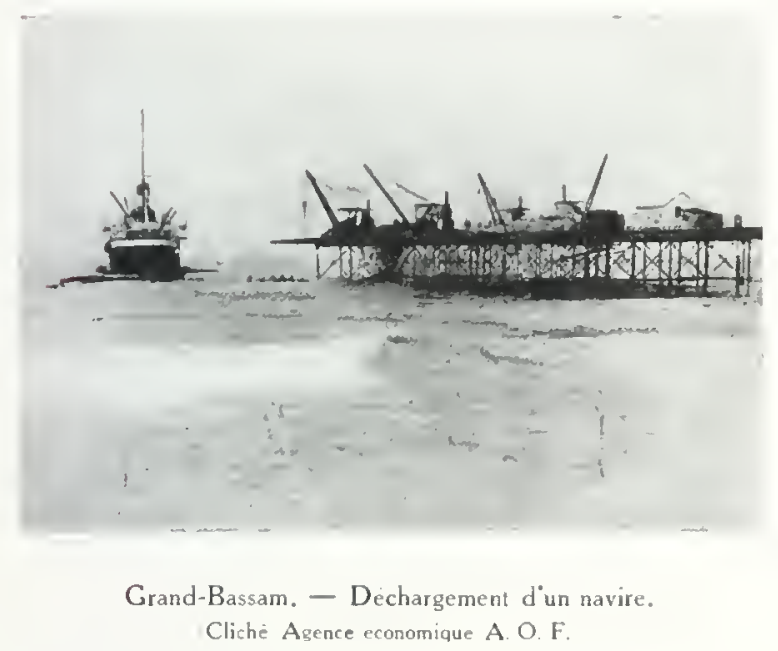

transformer nos plateaux en parquet, nous aurions à compter 15 à $18 \%$ de déchet, et 100 francs environ de frais de débitage par mètre cube. Il resterait encore une belle marge de bénéfice.

Les beaux cormiers en plateaux se vendent de 500 à 600 francs le mètre cube, aubier compris; le buis de 800 à 1.200 francs; les gaïaks sont beaucoup plus cher encore. Des bois comme le $F_{\mathrm{ou}}$, qui peuvent remplacer ceux-là, doivent donc pouvoir être vendus avec avantage aussi bien pour les employeurs que pour l'importateur.

Bref, l'exploitation des bois lourds à la Colonie peut prendre, étant conduite parallèlement avec celle des bois plus légers susceptibles de flotter, une extension considérable.

Elle est à développer pour les scieries déjà installées près de Bassam et d'Abidjan; elle doit être le complément de toute exploitation de bois ordinaires de menuiserie et de charpente et motiver, de ce fait, l'installation sur place de scieries nouvelles ayant, en Afrique occidentale même, l'écoulement de la majeure partie de leur production en sciages de qualité secondaire.

\section{ECOULEMENT ET PROPAGANDE. INTERVENTION DE L'ETAT.}

Laissons de côté les bois à traverses pour ne nous occuper ici que des essences importées en billes rou plateaux en France. Celles-ci peuvent soutenir la lutte contre les bois d'autres provenances et trouver écoulement, mais à une condition, c'est qu'on les fasse connaître au public. Ce n'est pas là besogne aussi facile qu'on pourrait le supposer à première vue. Certes, les Bois Coloniaux ont leurs apôtres et nous ne saurions trop reconnaître ici le mérite des louables efforts tentés par les Gillet, les Bertin, les Rouget, pour lancer les bois du Gabon et de la Côte d'lvoire dans l'industrie. Les Agences économiques des Colonies font également, dans les foires d'échantillons, une propagande extrêmement utile à cet égard (1). De résultat pratique il n'y en a guère eu jusqu'à présent, tout au moins pour la Côte d'lvoire. On a bien trouvé quelques acheteurs disposés à acquérir certaines quantités d'un ou plasieurs bois déterminés pour en faire l'essai; on n'a pu tronver d'exploitants ou d'industriels susceptibles de leur livrer ces bois autrement qu'en billes. Encore la plupart des essences que nous considérons comme intéressantes ne donnent lieu à aucune exploitation à la Colonie et il est matériellement impossible de se les procurer, à moins de les demander à l'avance et de les payer au prix des bois d'ébćnistcrie. Autrement fertile en résultats serait cette propagande si les agants qui en sont

(1) Celle de l'Afrique occidentale a obtenu à Paris. à Lyon et à Bruxelles notamment, de véritables succès avec les échantillons de hois de la Côte d"lvoire. 
chargés pouvaient dire aux employeurs : " $\mathrm{Ce}$ bois qui vous plait à divers points de vue et que vous seriez désireux d'expérimenter vaut, sec, tel prix, en telle dimension. Pour en avoir, adressez-vous à la maison X..., à tel endroit, qui vous livrera telle quantité qui vous plaira. " Ne serait-ce que par curiosité, qu'il se présenterait quantité de gens désireux de faire l'essai. Et d'essais nombreux pourraient résulter peu à peu quelques commandes importantes (I).

Cette propagande des Agences économiques, de même que celle entreprise par le Service des Bois coloniaux du Ministère des Colonies, est à continuer jusqu'à ce que la question de l'utilisation des Bois coloniaux soit complètement résolue. Outre qu'elle contribue beaucoup à faire connaitre ces bois au public, elle peut attirer sur eux l'attention de capitalistes disposés à entreprendre en grand leur exploitation et leur importation dans notre pays. Les demandes de renseignements reçues à ce sujet depuis dix-huit mois à l'Agence économique de l'Afrique occidentale, et dont certaines émanent de groupements importants, sont assez nombreuses pour démontrer que les efforts faits dans ce sens ne sont pas infructueux. Soit que la documentation fournie par l'Agence, pour être très complète en ce qui concerne les possibilités de la Colonie, n'ait pu porter sur toutes les questions qu' $j i$ nécessaire d'étudier à fond avant de se lancer dans une entreprise de cette envergure, soit que la crise commerciale qui dure depuis fin 1920 ait paralysé les initiatives, aucun des projets envisagés n’a cependant encore été réalisé. Mais la question a fait son chemin et nous voulons croire, d'autre part, sans fatuité ni fausse modestie, que le présent travail apportera, à tous ceux

(1) C'est ce procédé de vulgarisation que nous préconisions dans un rapport fourni à l'Agence économique de l'Afrique occidentale française, à la suite d'une des récentes foires d'échantillons. A défaut de maison pouvant vendre de petits lots de bois débités, nous envisagions la création d'un dépôt officiel de bois variés que l'Agence économique aurait vendus au détail et au prix de revient. que la question peut intéresser, certaines indications qui pourraient leur manquer.

$$
\text { " }
$$

"Les Bois coloniaux sont des intrus ", diton. "Personne n'en voudra, d'abord parce qu' on leur a fait mauvaise réputation, ensuite parce que le public (limité pour cet objet aux entrepreneurs de menuiserie, de charronnage et de constructions diverses, aux architectes pour le bâtiment) hésite toujours à employer une matière nouvelle dont il ignore les effets (1) . "Qu’à cela ne tienne! Rien n'empêchera les intéressés de pouvoir se livrer à tous les essais qu' ils jugeront nécessaires si nos bojs sont offerts, à qualité égale ou supérieure à première vue aux bojs habituellement employés, à meilleur compte que ces derniers et on peut croire que ces essais seront nombreux. Nous, nous sommes sûrs, de plus, qu'jls seront convaincants, qu'ils donneront toute satisfaction s'jls sont employés secs. Pour les architectes, la question est toutefois délicate car ils sont responsables, pendant dix ans, des travaux qu'ils font effectuer et de la qualité des matériaux qu'ils emploient. La seule appréhension d'être engagés dans des procès, si les bois utilisés par eux ne donnaient pas satisfaction à leur clientèle, pourrait les faire hésiter. Mais là encore nous avons à notre portée un moyen de dissiper cette appréhension. C'est à l'Etat que nous demandons de le fournir.

Ainsi que nous l'avons déjà fait observer, la question de l'utilisation des Bois coloniaux intéresse la Nation tout entière. L'Etat se doit donc d'aider à la diffusion de ces bois. Il peut le faire sous de multiples formes, soit en subventionnant les entreprises d'importation, soit en achetant lui-même des lots importants dont il a l'emploi pour les Administrations et pour la reconstruction des Régions dévastées, etc. Ecartons immédiatement la première; nos bois doivent pouvoir être vendus sans aide financière de l'Etat. Nous pouvons, par contre, envisager

(1) Rapport Weber. 


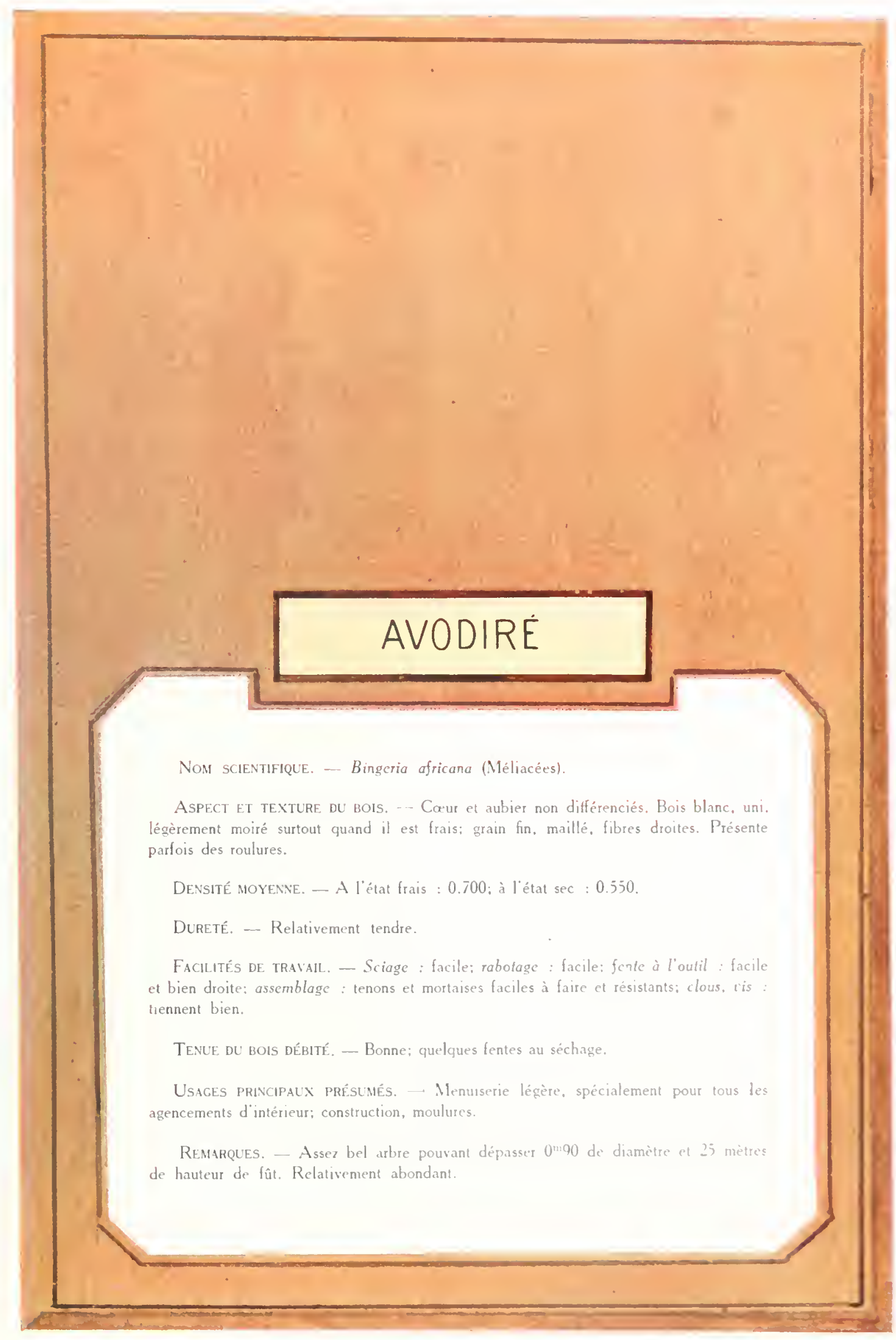



la seconde. Ce que l'Etat a fait sans hésiter pour les bois du Gabon, il peut le faire pour les bois de la Côte d'Ivoire. II peut d'autant plus le faire que les marchés qu'il pourra passer avec les premiers groupements décidés à entreprendre l'exploitation des bois de cette Colonie et leur importation en France, ne pourront présenter aucun aléa: on ne lui demandera pas, en effet, nécessaire dans ces bois aux particuliers, lesquels ne manqueront pas de les observer puis, s'ils $y$ trouvent un avantage, à les employer à leur tour.

L'Etat peut faire plus encore et sans que cela lui coûte rien. II faut qu'il inscrive ou fasse inscrire les bois de la Côte $\mathrm{d}^{*}$ lvoire, suivant leur classification, au nombre de ceux qui figu-

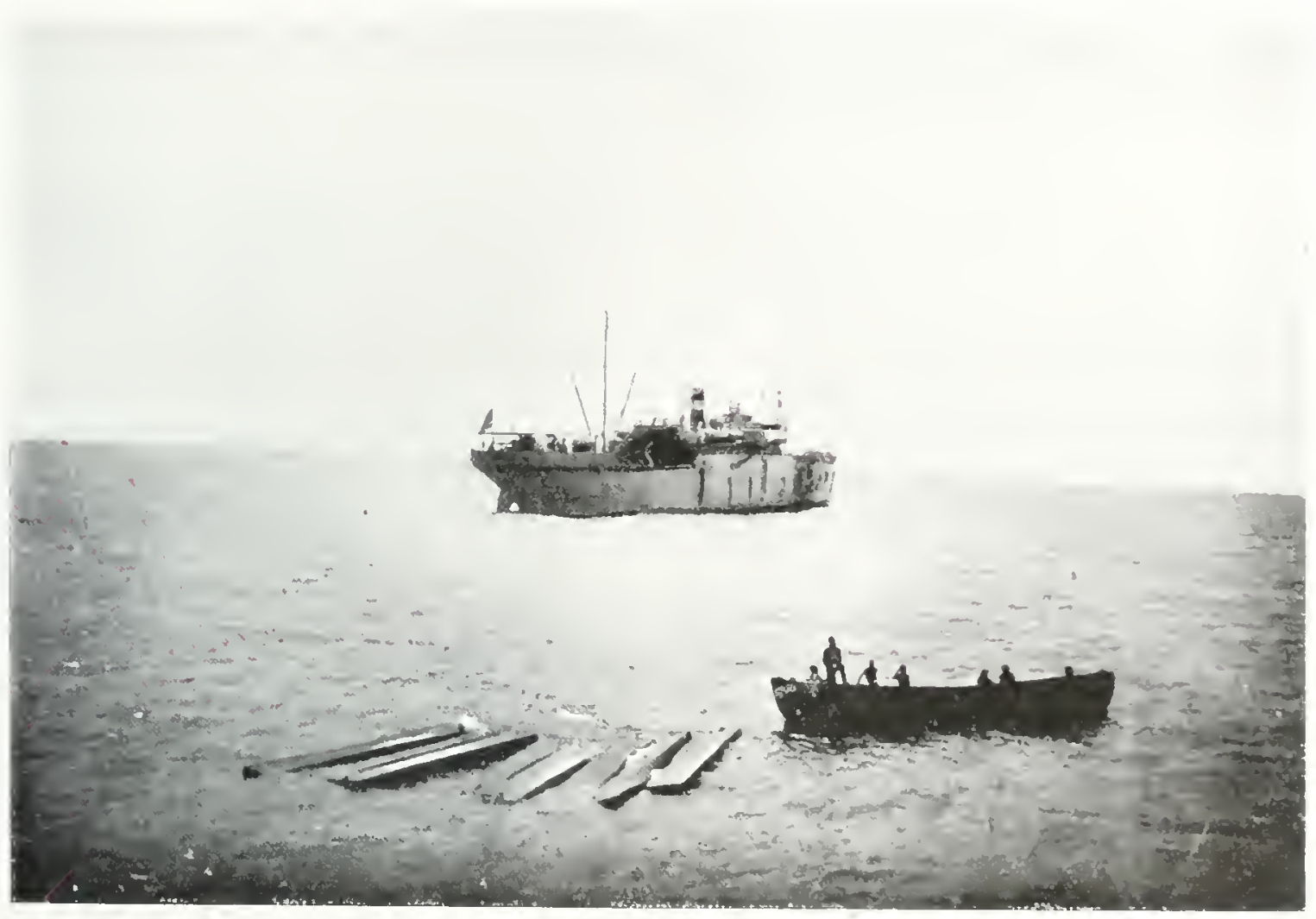

Entre le wharf et le cargo. - Embarcation dromant les billes. Cliché Mletayer. - Grand-Bassam

d'acheter des bois en billes pouvant donner des mécomptes au débitage ou au séchage, mais des bois débités, secs et garantis de bonne conservation. Ces marchés peuvent être conclus par avance. Dès l"instant qu'il ne paiera pas ces bois plus cher qu'il ne paierait les bois similaires français ou étrangers, l'Etat ne risque pas do faire une mauvaise affaire; il en fera faire une qui sera acceptable pour le fournisseur, une qui sera excellente à la fois pour la Colonie et pour la France. Il inspirera surtout la confiance rent dans les cahiers des charges relatifs aux adjudications publiques et les séries de prix des différentes Administrations (1). inscription qui aura pour avantage de vulgariser les noms de ces bois. lesquels sont bien barbares pour nos oreilles françaises, et d'en permettre officiellement l'emploi par les architectes et les entrepreneurs. Ce sera la meilleure propagande entreprise en leur faveur.

(I) Rapport W'eber. 
II faut, d'autre part, pour permettre aux bois de la Côte d"Ivoire de lutter à armes égales contre les bois d'autres provenances, les faire classer comme bois communs par toutes les Compagnies de chemins de fer (1) et ne conserver la classification de bois exotiques que pour les seules essences.

Les bois provenant de l'Afrique occidentale sont exempts de tous droits à leur entrée en France qu'ils soient débités ou non (2). C'est un avantage qu'ils offrent sur tous les bois du Gabon et du Cameroun, encore soumis au tarif minimum et qui doivent acquitter à leur entrée dans la Métropole (bois d'ébénisterie en billes exceptés) une taxe de 0 fr. 65 par 100 kilos pour les grumes, de 1 franc par 100 kilos pour les billes équarries et de 1 fr. 25 à 1 fr. 75 pour les bois sciés. Les bois d'ébénisterie sciés, provenant de ces Colonies, paient I franc par 100 kilos.

\section{COMMENT ORGANISER L'EXPLOITATION DES BOIS COMMUNS}

L'exploitation à la Colonie des bois de charpente et de menuiserie, l'importation en France, le débitage, le stockage et la vente de ces bois nécessitent donc une organisation assez complexe, qu'il ne parait possible de réaliser que par la formation d'une ou plusieurs Sociétés disposant de gros capitaux.

Car, on l'a déjà fait observer et le fait n'est pas discutable, une entreprise de cette naiure n'est susceptible de réussir que si elle prend dès son début une certaine ampleur. Nous n'avons parlé de 10.000 tonnes de bois, comme rendement minimum d̀ attendre d'un chantier bien organisé, que pour faciliter l'établissement de

(1) C'est déjà fait sur la plupart des réseaux.

(2) Les exemptions, sans limitation de quantités. comprennent les bois à construire et les "bois d'ébénisterie " (page $226 \mathrm{du}$ tarif). Ces deux dénominations peuvent s'appliquer à presque tous les bois importés de la Côte d'lvoire. Seuls les merrains et les traverses de chemin de fer, qui ont une destination bien spéciale. peuvent être soumis aux droits de 0 fr. 75 à I franc par 100 kilos. notre prix de revient. Mais il est nécessaire d'envisager une exploitation beaucoup plus importante et que nous n'estimons pas inférieure à 50.000 tonnes pour être tentée avec de réelles chances de succès. Et encore, si l'on devait en rester à ce chiffre, ce serait bien peu, étant donnée la variété des bois à importer, pour faire figure honorable parmi les bois d'autres provenances dont l'importation en France atteint des centaines de mille et même des millions de mètres cubes.

C'est donc, rien que pour l'exploitation, qui devra être entreprise avec un matériel perfectionné (1) pour réduire le plus possible la main-d'œuvre employée, un capital de deux à trois millions qui est nécessaire. Ne parlons pas du fret, il peut être payé à l'arrivée des bois. L'achat de terrains en France pour l'usine de débitage, le parc d'attente des billes et celui de séchage des bois sciés, les constructions, l'achat et I'installation de l'outillage, peuvent entraîner, d'autre part, une dépense de cinq à six millions, dont plus de deux millions rien que pour l'outillage et les machines. Ajoutons à cela qu'il nous faudra conserver pendant deux ans, avant de les vendre, la majeure partie des bois débités; le stock permanent pourra être par conséquent, pour une importation annuelle, de 50 à 60.000 mètres cubes de bois débités. de 70 à 80.000 mètres cubes de bois sciés (2), représentant une valeur, à 300 francs le mètre cube en moyenne, de vingt à vingt-quatre millions. I] est bien certain qu'étant assuré contre l'incendie, ce stock pourra être mis en warrant; il n immobilisera pas un capital en rapport avec sa valeur. C'est du moins un gros facteur dont il $y$ a lieu de tenir compte.

(I) L'emploi de scies actionnées par des moteurs, pour le tronçonnage et l'équarrissage, ne semble pas devoir être préconisé sur les chantiers d'Acajou, la grande dissémination des coupes obligeant à des déplacements constants et difficiles des appareils. Par contre, sur les chantiers de bois communs, ce matériel peut rendre de très utiles services et faire réaliser de sérieuses économies.

(2) Chiffres qu'on peut réduire considérablement si on dispose d’installations pour le séchage mécanique. 
Comme nous l'avons déjà exposé, il y a, d'autre part, intérêt pour toute entreprise d'importation de bois de la Colonie, à monter, à proximité des chantiers, sur une des voies navi-

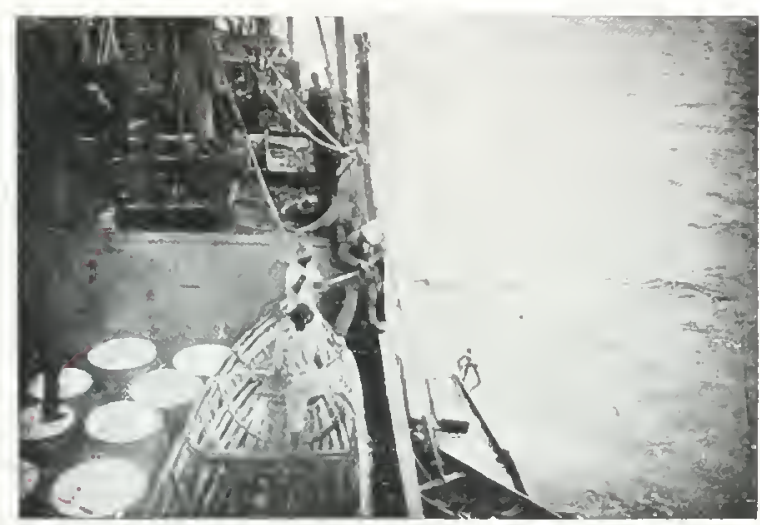

Le long du bord. - La bille est crochetée pour ètre soulevee. - Clichè Métayer. - Grand-Bassam

gables reliées au port d'embarquement, une scierie de moyenne importance (20 à 30.000 mètres cubes par an) pour débiter les bois durs (1), de densité supérieure à 1.000 et permettre d'envoyer en France, sous forme de plateaux, plus maniables que des billes entières, des lots sélectionnés d'Oboto, de Fou, de Tali, d'Adzobé, peut-être de Sougué, d'Adjouaba, etc., dont on pourra avoir le placement pour des usages spéciaux. Les parties moins bonnes, les dosses, seront utilisées pour de la traverse et des sciages à écouler en Afrique occidentale française même.

Cette scierie pourra également débiter en plateaux les bois sujets à échauffement, comme le Bahia et l'Avodiré, bois qu'il sera peut-être nécessaire, dans ce cas, de sécher ou desséver immédiatement.

Si sommairement qu'elle puisse être installée, cette usine, surtout si elle comporte des installations de séchage mécanique, pourra bien coûter près de deux millions de francs (2).

(1) Les bancs de scie, pour équarrissage des bois à expédier en billes, pourront être placés en dehors de l'usine.

(2) Nous avons vu plus haut que cette scieric pourrait peut-être utilement servir au débitage immédiat des bois sujets à échauffer.
Tout compte fait, l'organisation envisagée nécessite un capital de 10 à 12 millions pour être menée à bien.

Elle exige également des compétences, aussi bien pour la direction des usines de débitage que pour l'exploitation et l'évacuation des bois des chantiers.

$\mathrm{C}^{\prime}$ est une très grosse affaire, en somme, et qui ne peut être entreprise que par des hommes déjà familiarisés avec les questions coloniales et tout ce qui touche au commerce des bois. Seule unẹ Société puissante, ayanì à sa tête des spécialistes en chaque matière peut, après choix et obtention d'une zone de forêt assez étendue, avec étude très complèie des peuplements de cette zone, des possibilités d'évacuation des

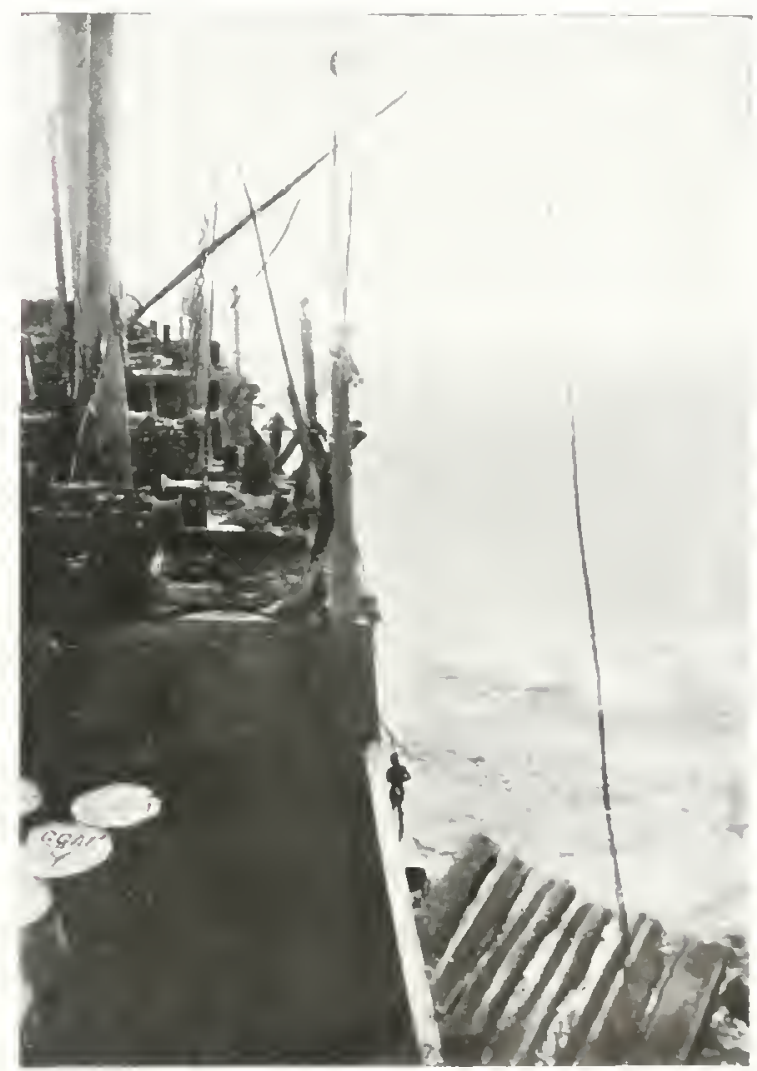

Une chaloupe du cargo a amene la drome le long du batcau Cliche Wetayzr - Grand Bassum

bois, se lancer dans une affaire de cette importance. Il ne parait pas moins indispensable, pour ĉtrc assuré de l'écoulement des premiers stocks 
de bois sciés en France, d'avoir préalablement obtenu de l'Etat une première commande importante, à livrer par échelons et à des prix correspondant à ceux qui sont pratiqués pour des bois similaires, prix rémunérateurs par conséquent, nous l'avons vu, pour la Société qui bénéficiera de cette commande.

Ces conditions réalisées, la réussite paraît certaine. Le placement des bois, une fois quelques livraisons faites à l'Etat, sera facile, pour peu que la Société complète la propagande offcielle qui doit être continuée par des envois gratuits d'échantillons et de prix-courants, consente à faire, au début, des livraisons minimes pour tous les essais que la clientèle désirera entreprendre. L'emploi des bois de la Colonie fera tache d'huile; ces bois s"imposeront peu à peu d'eux-mêmes dans toute la France, dépasseront peut-être les frontières.

L'amélioration des conditions d'embarquement résultant, par exemple, de la construction du nouveau wharf, à Bassam, l'emploi de toulines ou de câbles aériens pour le chargement rapide des bois en billes peut, d'autre part, amener rapidement une réduction sensible des tarif́s de fret, lesquels pèsent si lourdement sur les importations de bois de la Colonie.

La baisse des prix du bois en France n'est par ailleurs pas à craindre. Actuellement, les cours sont inférieurs à ce qu'ils devraient être réellement en tenant compte du change et des coupes excessives faites pendant la guerre. L'arrêt des constructions d'immeubles a réduit considérablement les besoins, de sorte que, si la production a été diminuée beaucoup depuis deux ans, elle a été néanmoins presque suffisante pour répondre à la consommation qui a diminué bien plus encore.

Les importations ont donc subi un arrêt, les cours se sont affaissés. Cela ne peut durer longtemps et bientôt les importations reprendront, dépasseront leurs chiffres d'avant-guerre; les cours s'en ressentiront. Le change, dût-il, d'autre part, s'améliorer notablement que ces chiffres n'en souffriront guère (1).

Une baisse sensible des prix de vente n'est donc pas à envisager avant longtemps. Elle peut se produire, mais alors elle sera la conséquence de l'abaissement général des salaires ou des tarifs de fret; les bois coloniaux, moins que ceux des autres provenances, moins que les bois indigènes, ont à la redouter.

(1) Les grosses différences de prix entre 1920 et 1921 résultent beaucoup moins, en effet, de l'amélioration du change que de la réduction de la consommation.

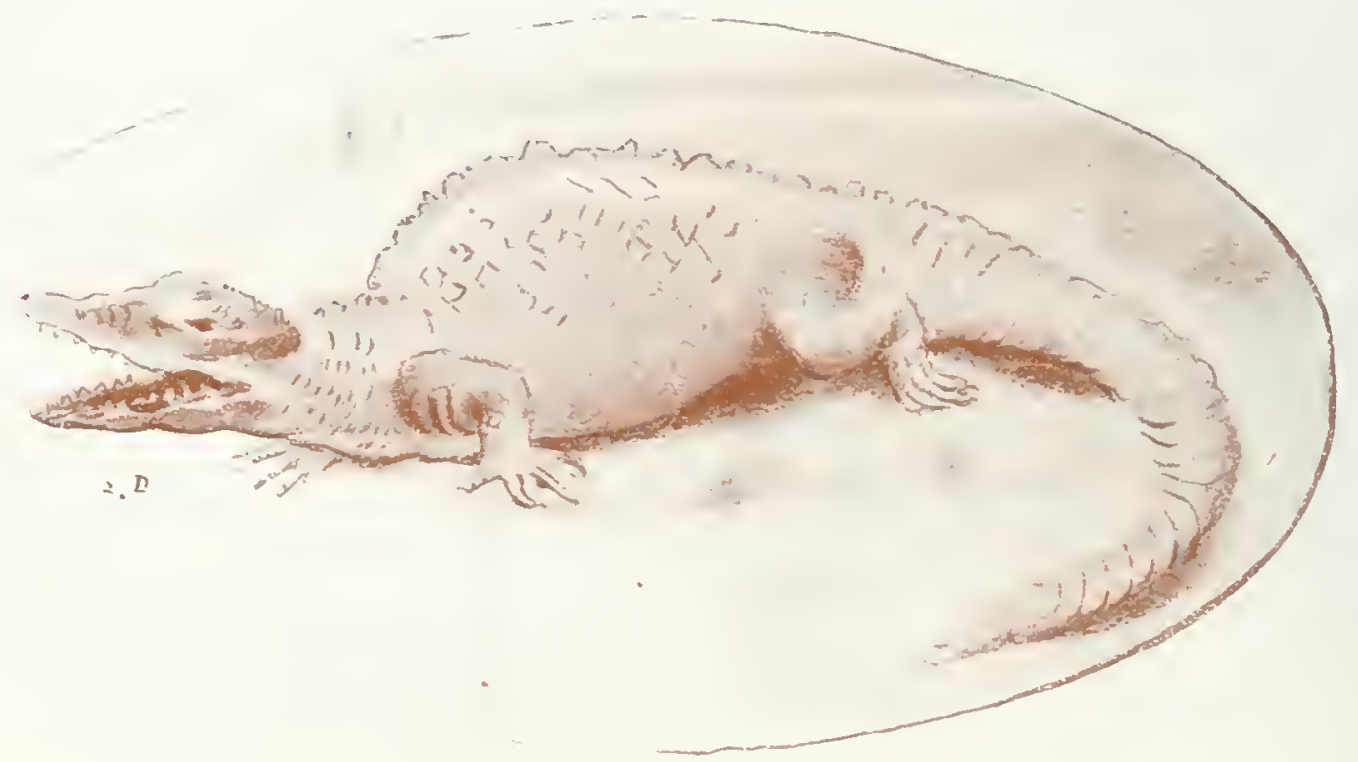




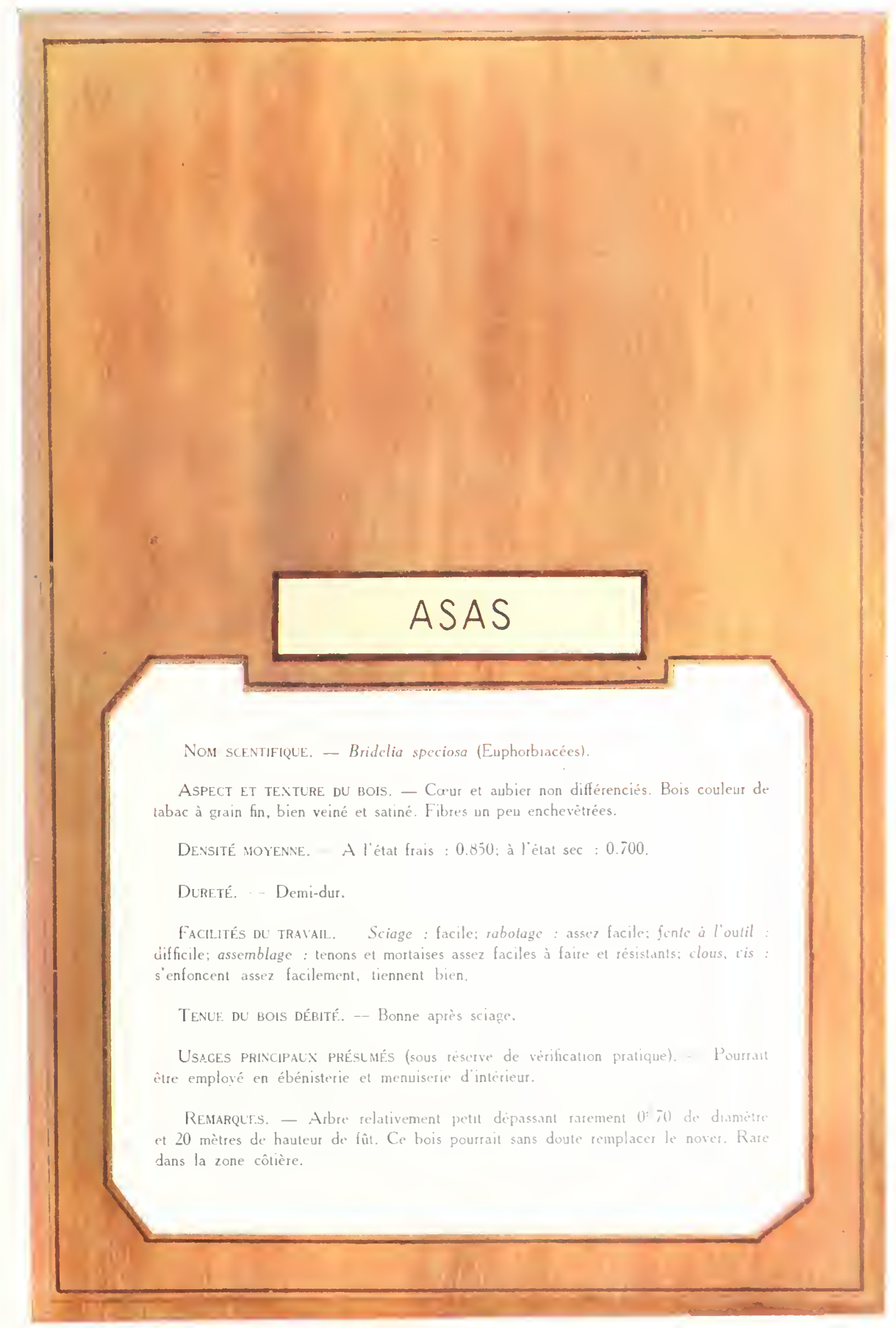


CONCLUSIONS 



\section{CONCLUSIONS}

L'exploitation de la forêt de la Côte d'lvoire, plus encore en ce qui concerne les bois communs qu'en ce qui concerne les bois d'ébénisterie, apparaît donc commme susceptible d'un développement important. En ne comptant que sur ce qui est facilement et immédiatement réalisable, il n'est nullement exagéré d'affrrmer qu'il pourrait être sorti annuellement, et d'une façon suivie, du domaine forestier de la Colonie, 100.000 tonnes de bois d'ébénisterie et 200.00 tonnes, au moins, de bois de menuiserie, charpente, bois à traverses, etc., pour peu qu'on veuille réellement perfectionner les moyens et les méthodes d'explaitation des premiers, pour peu qu'on veuille entreprendre franchement celle des seconds.

Ces trois cent mille tonnes de bois représenteraient déjà un chiffre intéressant. Leur exploitation, rien que pour la main-d'ouvre et les taxes encaissées par l'Administration, mettraient chaque année, dans la Colonie, 10 à 12 millions de francs d'argent nouveau, dont bénéficieraient le commerce, la colonisation et l'outillage économique du pays. Elles représenteraient pour notre marine un appoint intéressant; pour la France, ce serait 300.000 tonnes de bois, d'une valeur de soixante à quatre-vingts millions de francs (1), à importer de moins de l'étranger. C'est peut-être très peu de chose si l'on tient compte que c'est par milliards de francs que nous devons acheter à l'extérieur les produits et marchandises qui manquent à nołre industrie. Si peu que ce soit, ce n'est pas négligeable. Et puis, cela peut n'être qu'un commencement. La construction d'un port dans la région de Bassam-Abidjan, la multiplication des wharfs sur les autres points de la côte, l'aménagement des roies fluviales, la construction de nouveaux chemins de fer de pénétration partant de la côte, tout cela constituera pour l'avenir des facteurs de développement pour les exploitations forestières, de même que pour la mise en valeur du pays. Tout cela sera aussi la conséquence d'un premier développement de ces exploitations. Il s'agit donc de commencer san: plus attendre.

\footnotetext{
(1) Valeur, avant débitage en France.
} 



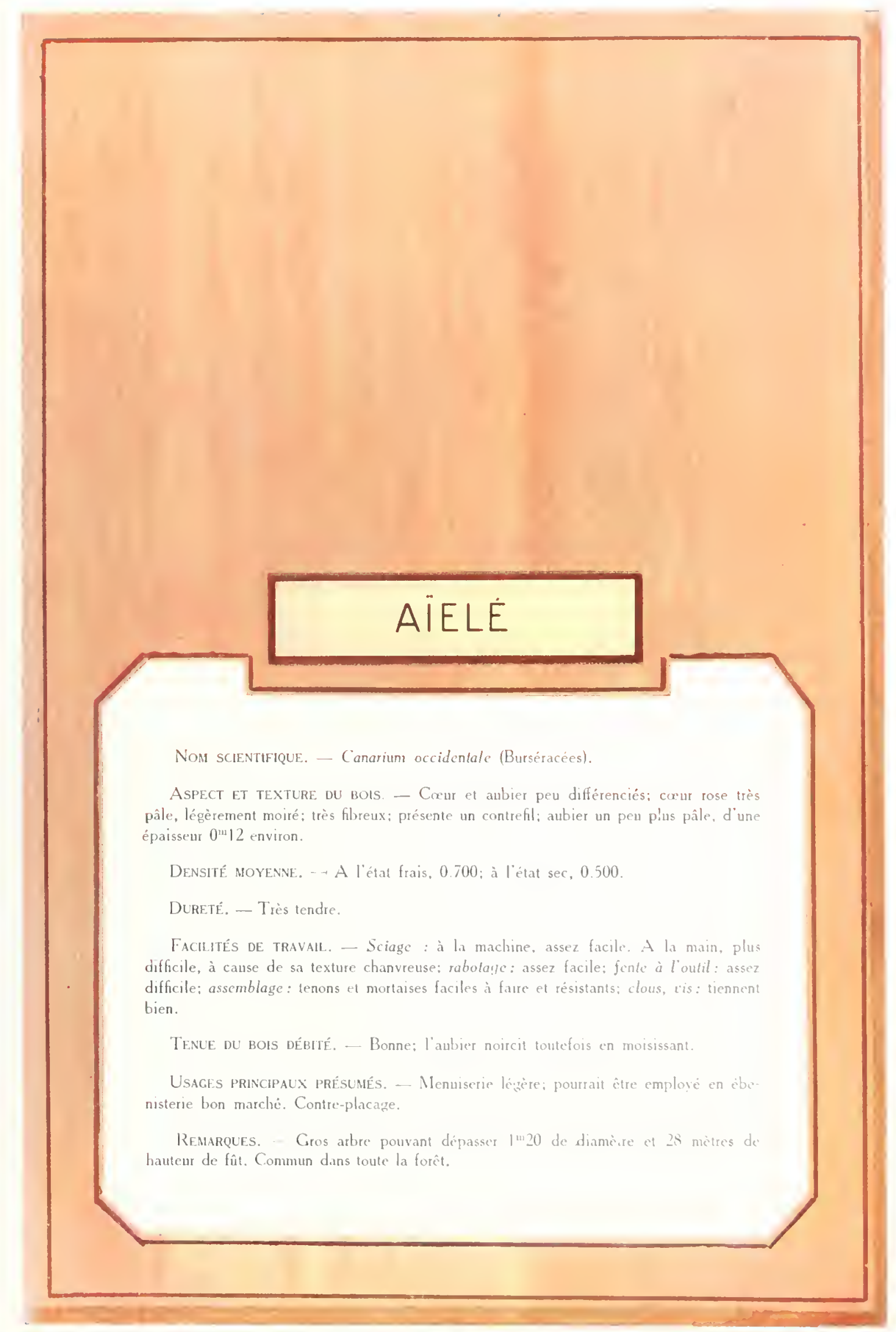


. 


\section{APPENDICE}

Utilisation éventuelle des essences tendres pour la pâte à papier

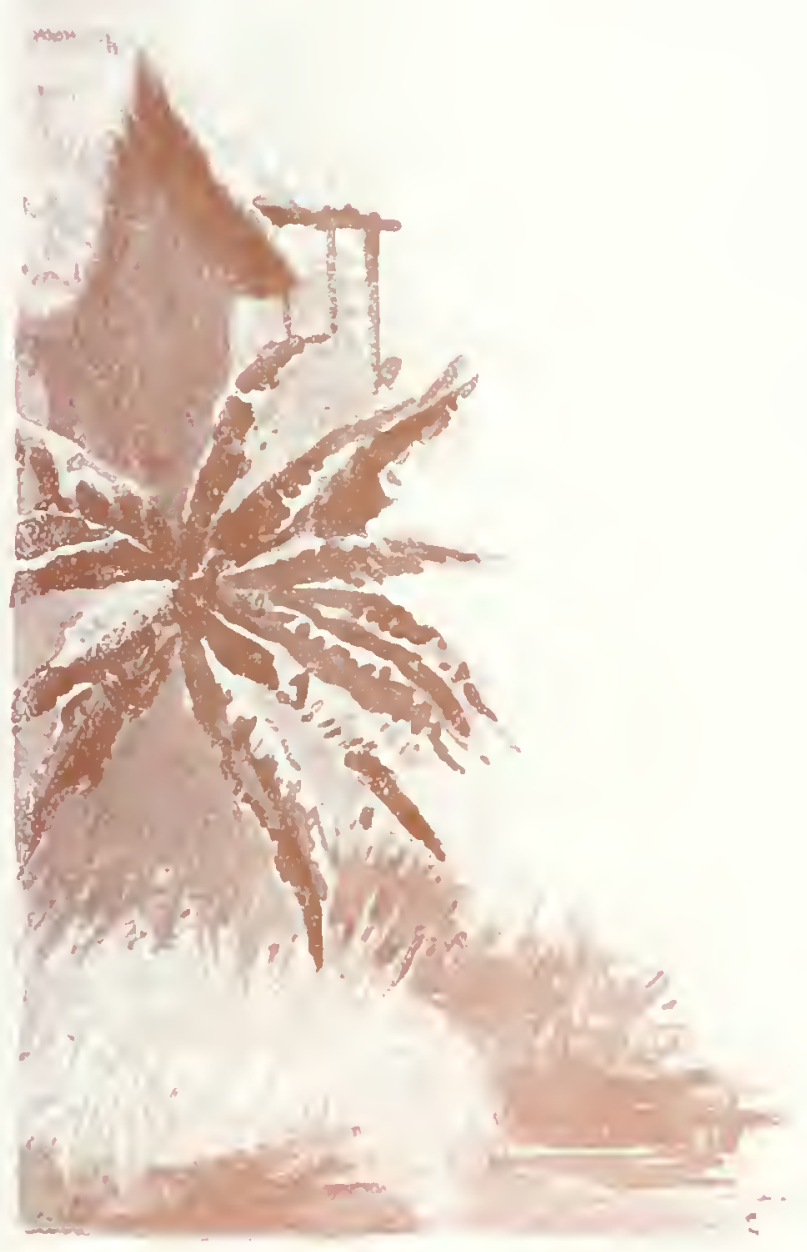





\section{APPENDICE}

\section{Utilisation éventuelle des essences tendres pour la pâte à papier}

L'utilisation, par des industries de produits chimiques, des déchets de bois divers, des essences dures ou demi-dures exploitées pour d'autres usages, exige au préalable des études techniques que seuls des spécialistes peuvent mener à bien. C'est un sujet que nous ne pourrons en conséquence qu'effleurer, nous bornant à signaler l'intérêt qu'il peut présenter pour l'avenir.

Nous ne sommes pas beaucoup plus compétents pour traiter de la fabrication de la pâte de bois et de l'utilisation, pour cette industrie, des essences tendres abondant dans la région côtière de la Colonie.

Pourtant, nous voudrions attirer tout spécialement, sur ce point, l'attention des fabricants français de papier ou de pâte à papier.

Toute la zone de forêt immédiatement en bordure des lagunes, zone qui a été la plus ravagée par les défrichements des indigènes, est peuplée, en majeure partie, d'essences très tendres, Parasoliers et Fromagers notamment. Ces essences existent également ailleurs en peuplements aussi importants sans doute. mais nulle part leur exploitation ne serait aussi facile, aussi peu onéreuse qu'auprès des lagunes.

Déjà, on s'est préoccupé de la question. Le Gouvernement local a concédé, en 1921, l'exploitation de la zone de forêt entourant toute la lagune Aby, sur une profondeur de I kilomètre.
Qu'en résultera-t-il ? On ne peut le prévoir encore. Une industrie de pâte à papier ne s"improvise pas; elle demande des études et une mise au point extrêmement sérieuses.

Quoiqu'il en soit, il y a un premier pas de fait. Et nous espérons qu' on ne s'en tiendra pas là. Il y a une zone plus intéressante, à notre aris. que celle baignée par la lagune $\mathrm{Abr}$ : c'est celle qui borde les lagunes Ebrié. Ml'Potou et la partie navigable du Comoë. Elle est beaucoup plus vaste que la précédenie: elle est desservie par Bassam, c'est-à-dire par un wharl. ce qui est appréciable: avant vingt-cing ans. elle le sera par un port.

C'est dans le roisinage d'Abidjan. de préférence dans une des îles de la lagune Ebrié, que devrait être installée une usine pour la fabrication de la pitte de bois. Déjà dans cette partie de la lagune, l'eau est à peine salée, elle y est limpide presque toute l'année et n'est lérèrement trouble qu au moment des fortes crues. le très faible courant Ouest-Est permettant aux matières organiques. amenćes par les rivières. de se déposer assez rapidement. Enfin. si le port est construit. comme il est probable. à Abidjan au lieu de Bassan. l'usine se trouvera dans le voisinage immédiat du déboushé maritime de la Colonie.

Nous estimons que less essences. conme le Parasolier et le Fromager, coupées dans une 
zone de 1 kilomètre, profondeur moyenne. en bordure du réseau navigable auquel nous faisons allusion, ne reviendraient pas en moyenne, rendues à l'usine, à plus de 12 francs le mètre cube.

La mise à l'eau, le remorquage à l'usine, sont en effet excessivement faciles; abatage, tronçonnage, manipulations sont également beaucoup plus rapides que pour les autres essences, étant donné que celles-là sont excessivement tendres, excessivement légères $(0,270$ densité moyenne du Parasolicr: 0,315, celle du Fromager). L'un et l'auire de ces bois peuvent fournir une excellente pâte à papier: le premier donne plus de $400 / 0$ de son poids total en cellulose, le second, 30 à $330 / 0$.

Autre détail à noter : la rapidité phénoménale avec laquelle croissent ces essences permet d'envisager, non seulement leur propagation naturelle certaine dans la zone exploitée, mais des plantations en terrain non boisé, plantations pouvant être exploitées tous les 10 ou 15 ans et susceptibles de faciliter considérablement le ravitaillement d'une usine de pâte à papier en matière première (1).

J. M.

(I) L'ingénieur agronome Ammann, actuellement en mission à la Côte d'Ivoire, doit étudier de très près cette question.

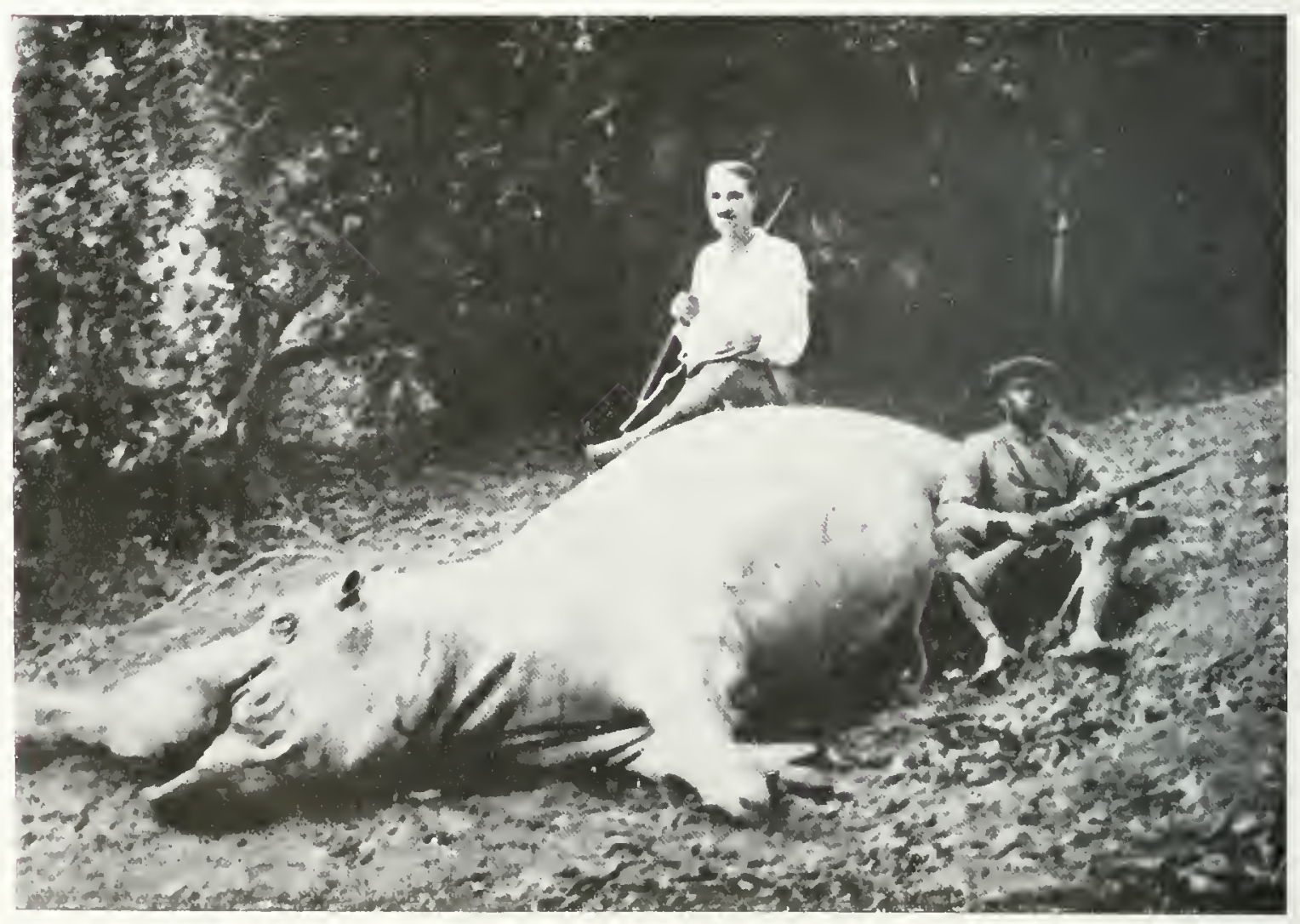

Chasse à l'hippopotame.

Cliché Burzer 


\section{TABLE DES MATIERES}

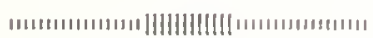





\section{TABLE DES MATIERES}

AverTIS:EMENT

Préface

INTRODUCTION

\section{Considérations générales sur le pays}

et ses habitants.

\section{Geographie}

Orographie - Hydrographie

Climatologie

Flore. Faune

Population - Main-d'cuvre

Commerce

Relevé des principaux produits exportes en 1919 el en 1920

Centres commerciaux.

Règime douanier .

Industrie .

Colonisation. Régime des Concessions

Moyens de communication

Relations avec l'Europe

Tableau des principales maisons de commerce, industries, banques, plantations installees a la Cóte d'Ivoire.

\section{TITRE PREMIIER}

\section{La Forêt.}

Conditions de son exploitation.

Ce qu'est la forèt de la Cöte d'Tuoire

Classement industricl

Tableanx des coefficients de trequence obtenus dans les prospections de la mission Bertin 27, 28 et 29

Pages 
TITRE $\|$

Les exploitations actuelles.

Bois précieux et bois communs.

Les exploitations actuelles.

Methodes d'exploitation. Défauts et avantages

Capital nécessaire à une exploitation d'acajou .

Prix de revient et prix de vente des bois exploités.

Prix de vente des bois exploites dans les chantiers d'acajou

Autres essences d'ebenisterie exploitees

Productions et débouchés

Qualité des acajous de la Cóle d'lvoire. Leur emploi. Tiama et lroko.

Acajous de choix, frises. mouchetés ou "figurés"

Acajou-Tiama et Iroko.

Utilité pour les exploitants de ne presenter que des bois de toute premiere qualité

Les scieries locales. Leur organisation. Ecoulement de leur production

\section{TITRE [I]}

\section{Avenir réservé au $\mathrm{x}$ bois communs. \\ Leur exploitation intensive \\ et leur importation en France.}

Ecoulement en Europe des bois de la colonie autres que les bois d'ébénislerie et de grand choix

Valeur des bois coloniaux en général

Essences à exploiter à la Côle d'luoire et à importer en France.

Bois d'ebénisterie (exploitation accessoire).

66

66
Bois de menuiserie et de charpente de densité inférieure à 1.000

Pages

Bois semblant convenir plus particulierement pour l'outillage.

Bois de densite supérieure a 1.000 convenant pour la charpente lourde. le pilotis. la traverse de chemin de fer

Récapitulation des essences autres que celles d'èbénisterie (tableau). . . . .

\section{PLANCHES HORS TEXTE}

Variété d'acajou

Village au bord de la lagune (aquarelle)

Le Comoë (aquarelle)

Acajou

Tiama

Iroko

Makoré

Niangon

Badi

Bossé.

Dabéma

Bahia

Rikio

Sougue
Pages

XIII

19

23

27

31

35

39

43

47

51

55

59

63
Présentation des bois examinés au précédent chapitre. . . . . . . . . .

Présentation. . . . . . . . 94

Sciage et sechage . . . . . . . . . 96

Prix de revient et prix de vente possibles des bois importés de la colonie. .

Bois de densite inférieure à 1.000 . . . 98

Bois de densité supérieure à 1.000 . . 107

Ecoulement et propagande. Intervention de l'Elat. . . . . . . . . 109

Comment organiser l'exploitation des bois communs. . . . . . 112

CONCLUSIONS

APPENDICE.

Utilisation éventuelle des essences tendres pour la pâte à papier.

\section{CARTES}

La Cóte d'Ivoire (Carte au $4.000 .000^{\circ}$ ) . VII

Canal de jonction des lagunes. . . . . . . 15

Relations maritimes françaises. . . . . . . 17

Carte forestiere de la Cóte d'lvoire . . . . 21

Grand-Bassam . . . . . . . . . . 46

Carte forestière de la Côte d'Ivoire . . . . . 53

La forêt de la Cöte d'Ivoire. Règion Sud-Est . 75

Framire

Pages

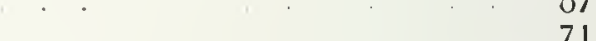

Apome $\quad 0 \quad 75$

Bodioa $\quad . \quad . \quad 79$

Tali _. . . . . . . 83

Adzobé _. . . . . . . 87

Fou . . . 9 91

Oboto $\quad . \quad . \quad . \quad 95$

Abalè $\quad . \quad . \quad . \quad 099$

Senan ......... 103

Olon . . . . 107

Avodire _.......... 111

Asas . . . . . 115

Aièlè . . . . . . . 119 


\title{
PUBLICATIONS COLONIALES
}

\author{
Ouvrages déjà parus
}

La pacification de la Côte d’Iroire, méthodes et résultats, par G. Awor LYAN, Gouverneur Général des Colonies. Lettre-préface du Général Guluien, Ministre de la Guerre (1016) in- $\delta^{\circ}$ arec cartes.

La Côte d'Ivoirc. - Le pays, les hal,itants, par G. Josepr, licencié és-sciences, administrateur des Colonies, préface de 11 . le Gourerneur Avgoultaxt (1917), in-so avec reproductions photographiques et cartes.

La Cote d'Ivoire, agricullure, commerce, intuslite. - Questions diconomiques, par Louis Le BARr, ItR; préface de Gabriel Bonvalot (1914), 1 rolume in-so illustré.

Mission Forestière Coloniale, envorée dans les Colonies françaises par les Ministeres de la Guerre, de l'Armement el des Colonies.

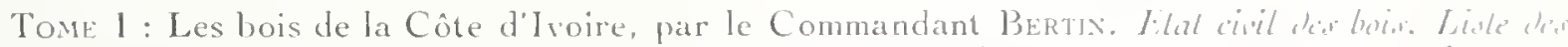

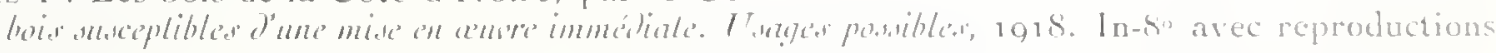
et cartes.

Tone 11 : Les bois du Gabon, 1918. In-ko arec reproductions photographiques el cartes.

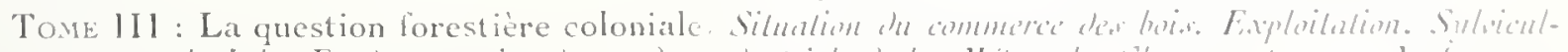

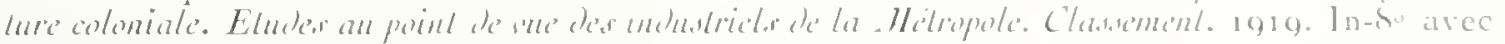
reproductions photographicues et cartes.

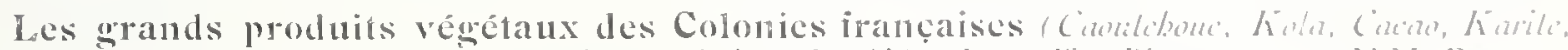

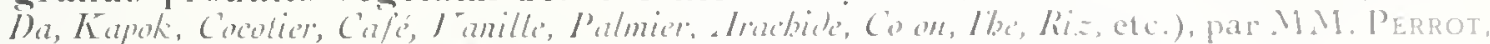
Vullet, Fauchere, Gatin, Anam, Man, Capus (197i). In-sillustré.

Irrigations ef cultures irriguées en Afrique tropicale, par Y. IlkRY, ingénieur agronome, directeur de l'Agriculture aux colonies, aree la collaboration de I. LEMart, ingénicur agronome. Un volume in-so, cartes et gravures. 1918.

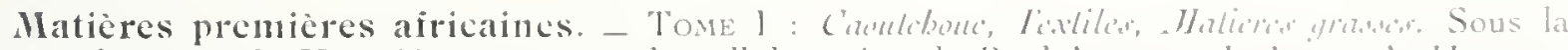

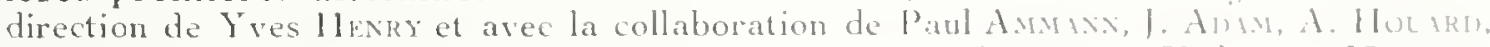

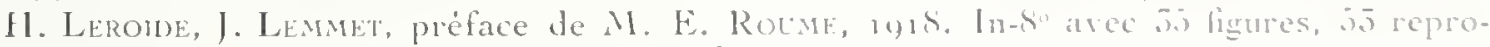
ductions photographiques et $\&$ cartes en couleurs.

L'Outillage écononnique des Colonies Fräluçáses, par M. Plin, ingénieur des "lratrux publics au Ministere des Colonies (1915), intis

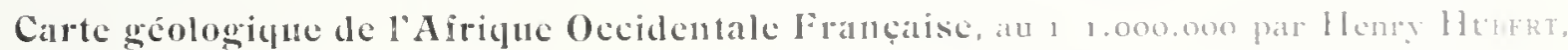
administrateur des Colonies.

Feuille 10: Bingerville (C'ile drovire) (101,-), aree note explicative.

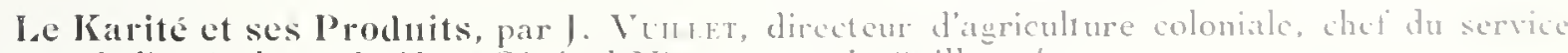
de l’agriculture du Haut-Sénégal-Niger. 1911, ln-s illustré.

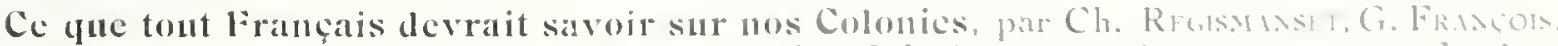
F. Rouget, chefs de hureau au Ministere des Colonies (1918), in-10 avec reproductions photographiques et cartes.

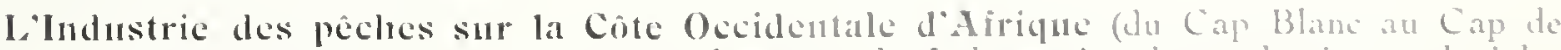
Bonne lispérance), par A. Grever, directeur du Lahoratoire de productions coloniales d'origine animale. 

IMPRIMERIE des ÉtAablisSEMENTS L. DUFAY' 3. Place de la Madeleine, 3 PARIS

1922 




DATE DUE

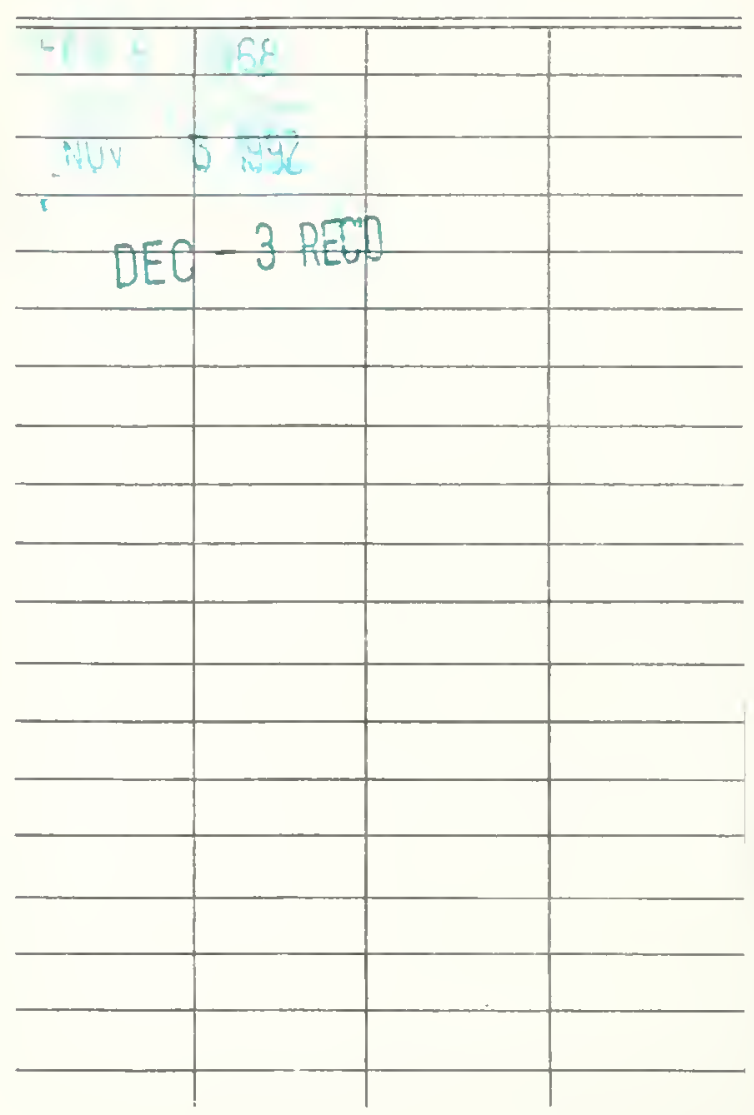


AGRICULTURE FCRESTR'í LIB!ARY

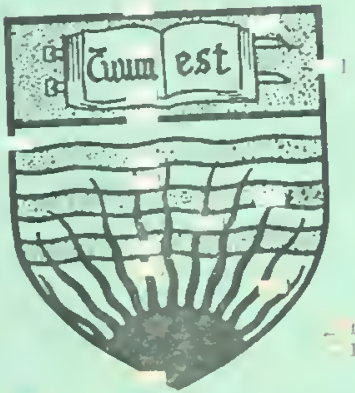


